\title{
Strength evaluation of honeycomb FRP sandwich panels with sinusoidal core geometry
}

An Chen

West Virginia University

Follow this and additional works at: https://researchrepository.wvu.edu/etd

\section{Recommended Citation}

Chen, An, "Strength evaluation of honeycomb FRP sandwich panels with sinusoidal core geometry" (2004). Graduate Theses, Dissertations, and Problem Reports. 2626.

https://researchrepository.wvu.edu/etd/2626

This Dissertation is protected by copyright and/or related rights. It has been brought to you by the The Research Repository @ WVU with permission from the rights-holder(s). You are free to use this Dissertation in any way that is permitted by the copyright and related rights legislation that applies to your use. For other uses you must obtain permission from the rights-holder(s) directly, unless additional rights are indicated by a Creative Commons license in the record and/ or on the work itself. This Dissertation has been accepted for inclusion in WVU Graduate Theses, Dissertations, and Problem Reports collection by an authorized administrator of The Research Repository @ WVU.

For more information, please contact researchrepository@mail.wvu.edu. 


\title{
Strength Evaluation of Honeycomb FRP Sandwich Panels with Sinusoidal Core Geometry
}

\author{
by \\ An Chen

\begin{abstract}
Dissertation submitted to the
College of Engineering and Mineral Resources

at West Virginia University

in partial fulfillment of the requirements

for the degree of
\end{abstract}
Doctor of Philosophy
in
Civil Engineering

\author{
Julio F. Davalos, Ph.D., Chair \\ Bruce Kang, Ph.D. \\ Jacky C. Prucz, Ph.D. \\ Pizhong Qiao, Ph.D. \\ Samir N. Shoukry, Ph.D.
}

\section{Department of Civil and Environmental Engineering \\ Morgantown, West Virginia \\ 2004}

Keywords: Strength Evaluation, HFRP Sandwich Panel, Analytical Solution, FE Analysis, Experimental Investigation

Copyright $@ 2004$ An Chen 


\title{
Strength Evaluation of Honeycomb FRP Sandwich Panels with Sinusoidal Core Geometry
}

\author{
An Chen \\ Advisor: Dr. Julio F. Davalos
}

\begin{abstract}
:
In recent years, the demands in civil infrastructure have provided opportunities for development and implementation of Honeycomb Fiber-Reinforced Polymer (HFRP) sandwich panels, both in rehabilitation projects and new constructions. The concept of lightweight and heavy duty HFRP panels, with sinusoidal wave core configuration in the plane extending vertically between face laminates, was introduced for highway bridge decks. This product was successfully implemented in several bridge projects.
\end{abstract}

The development of standards and guidelines is needed in order to promote wider acceptance of composite sandwich products in construction. Characterizations of stiffness and strength properties are necessary to facilitate the development of design guidelines. Much effort has been devoted to the modeling and optimization of the HFRP panel. This dissertation is the first study on strength evaluation of HFRP sandwich panels with sinusoidal core geometry. Core materials for sandwich structures are primarily subjected to out-of-plane compression and shear. Therefore, three major contributions are included in this dissertation: out-of-plane compression, out-of-plane shear, and study on facesheet laminates. All studies are carried out through a combination of analytical solution and experimental investigation.

Two analytical models, corresponding to pure compression and elastic buckling failure, respectively, are provided for panels subject to out-of-plane compression. The facesheet and core are attached by contact molding and are, therefore, not rigidly connected. Thus, the buckling problem can be described as the instability of an FRP core panel with two rotationally restrained loaded edges. An elastic restraint coefficient is introduced to quantify the bonding layer effect between the facesheet and core, and a simple and relatively accurate test method is proposed to obtain the restraint coefficient experimentally. By solving a transcendental equation, the critical compression buckling stresses are obtained, and a simplified expression to predict buckling strength is formulated in terms of the elastic restraint coefficient. The analytical solution is verified by Finite Element (FE) analysis. Compression tests were carried out to evaluate the effect of the bonding layer thickness and core thickness, and the experimental results correlate closely with analytical and FE predictions. A parametric study is conducted to study the 
core aspect ratio effect on the buckling load and design equations are provided to calculate the compressive strength.

Most of past studies in the area of out-of-plane shear are focused on stiffness only. As a step further, analytical models, including shear crushing, shear buckling, and delamination considering skin effect are provided. Two factors are addressed that contribute to the skin effect: shear and bending warping. A closed-form solution, based on proper description of displacement field at the interface, is derived considering shear warping. The accuracy of this method is verified by FE results. The FE model is then applied to study bending warping effect. The stiffness and the stress distribution subject to skin effect are presented. Critical parts are identified and suggestions for future design considerations are given. Based on the stress distribution, design formulas for delamination and shear failure are presented. Rayleigh-Ritz method is employed to study the shear buckling of core panels with two sides elastically restrained. Four-point bending tests were carried out according to ASTM standards to study shear strength and shear stiffness of the core materials. Two types of beam samples were manufactured by orienting the sinusoidal wave: (1) along the length ("longitudinal"), and (2) along the width ("transverse"). Design equations are provided to predict the failure load due to different failure modes and good correlations are obtained.

Strength properties of the facesheet are studied in detail to develop an optimized facesheet configuration. A progressive failure model is developed using FE method to predict the behavior of laminated composite plates up to failure, where the failure criteria are introduced through prescribed user defined subroutines. The accuracy of the model is verified through correlations between FE results and existing experimental data. This model is then applied to carry out a parametric study on facesheet. Three variables are included: material properties, including bidirectional stitched fabrics, unidirectional layer of fiber roving and chopped strand mat; layer thickness; and layer sequences. The quality of each alternative is evaluated based on stiffness and strength performance. In order to further investigate the behavior of facesheet experimentally, coupon samples on selected configurations to evaluate compressive and bending strengths were tested in accordance with ASTM standards. The strength properties both in the longitudinal and transverse directions were evaluated. The test results are also used to validate the progress failure model developed in this study. Through this combined experimental and analytical study, the strength properties of facesheet are obtained, which permits the optimization of facesheet design.

Finally, step-by-step design guidelines are provided, and examples are given illustrating the use of these guidelines. Recommendations to improve the strength of HFRP panels are presented. All the methods presented in this dissertation can be extended to study other types of FRP sandwich structures. 
To my beloveds:

\section{Lili Yang \\ Yuntong Chen \& Shulan Wang \\ Guodong Yang \& Delan Wang \\ Hong Chen \\ Sheng Chen}




\section{ACKNOWLEDGEMENTS}

I hereby express my sincere and deep gratitude to my advisor and committee chairman, Dr. Julio F. Davalos, for his continuing assistance, support, guidance, understanding and encouragement through my graduate studies. His trust, patience, knowledge, and great insight have always been an inspiration for me. Special thanks are given to the committee members, Dr. Bruce Kang, Dr. Jacky C. Prucz, Dr. Pizhong Qiao and Dr. Samir N. Shoukry for their interest in my research and careful evaluation of this dissertation. I am also grateful to Dr. Pizhong Qiao for valuable discussions and suggestions.

Laboratory assistance of Mr. Justin Robinson, Mr. Vinod Ramayanam and Mr. Avinash Vantaram should be acknowledged. I also want to thank my former colleague Dr. Weiqiao Wang for helpful discussions. Thanks are also expressed to Mr. David Turner and Mr. William J. Comstock for helping prepare the fixtures and specimens needed for the research.

This work is supported by NSF Partnerships for Innovation program and the WVU Res. Corp. The samples for this study and technical advice were generously provided by Dr. Jerry Plunkett of Kansas Structural Composites, Inc., Russell, Kansas, USA. The support is greatly appreciated.

Above all others, special thanks go to my wife Lili Yang, for her love, encouragement and unending support. I also must thank my parents and family for their everlasting support and blessings in my life. 


\section{TABLE OF CONTENTS}

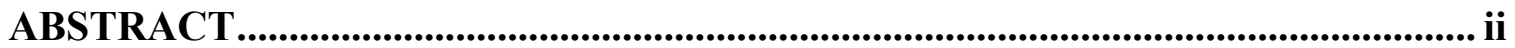

DEDICATION ........................................................................................................................ iv

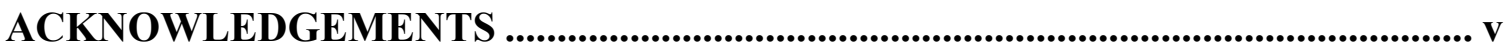

TABLE OF CONTENTS .................................................................................. vi

LIST OF FIGURES ............................................................................................................ $\mathrm{x}$

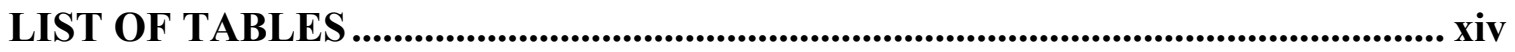

ABBREVIATIONS .......................................................................................................... xvi

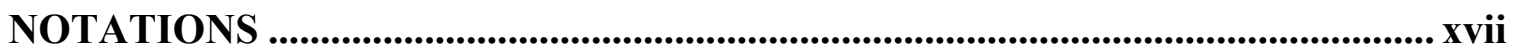

CHAPTER 1 INTRODUCTION _...................................................................... 1

1.1 Problem Statement and Research Significance............................................... 1

1.1.1 Development of Sandwich Structures.................................................... 1

1.1.2 Application in Civil Infrastructure...................................................... 4

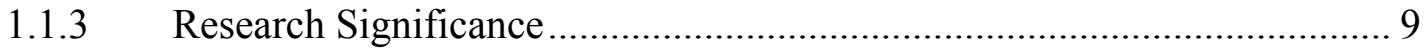

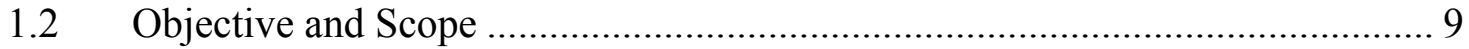

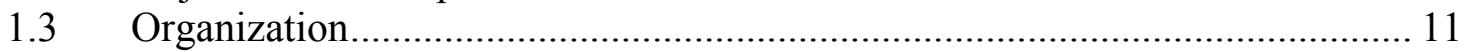

\section{CHAPTER 2 LITERATURE REVIEW ................................................................... 13}

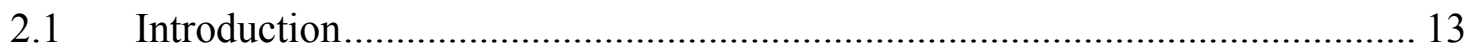

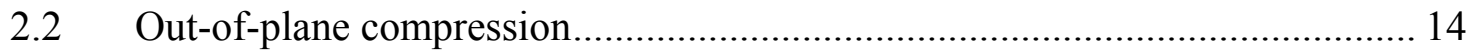

2.2.1 Compressive Strength of Core Materials ................................................. 14

2.2.2 Core Crushing ............................................................................. 15

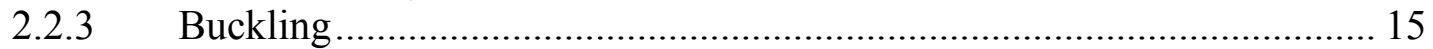

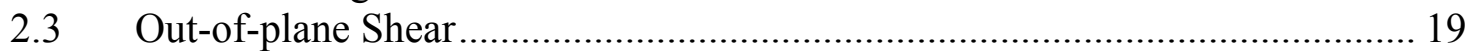

2.3.1 Stiffness Study on Equivalent Properties of Honeycomb Cores .............. 20

2.3.2 Interfacial Stress Distribution ............................................................ 23

2.3.3 Shear Crushing and Shear Buckling .................................................... 24

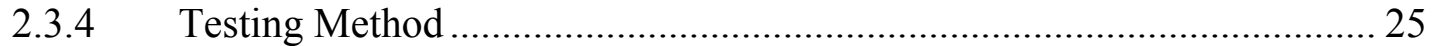

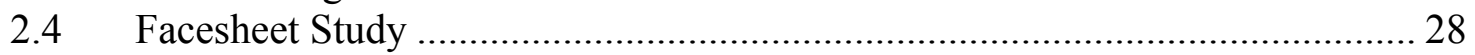

2.4.1 Progressive Failure Analysis.............................................................. 28

2.4.2 Testing Method .............................................................................. 32 


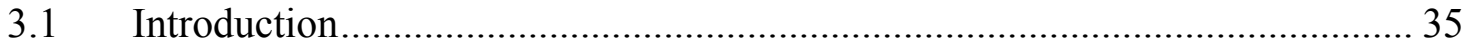

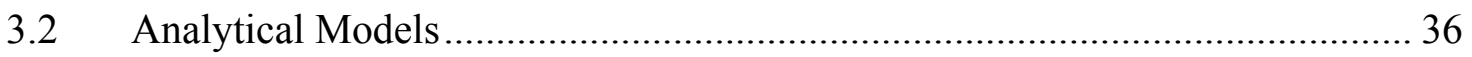

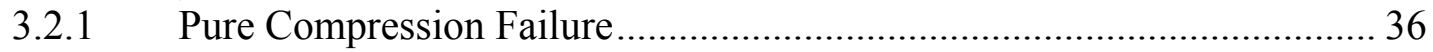

3.2.2 Buckling of Plate with Partially Constrained Loaded Edges..................... 37

3.3 Experimental Investigation .......................................................................... 49

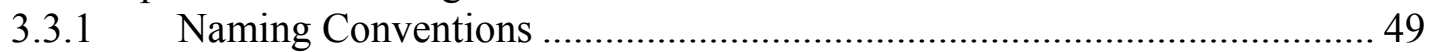

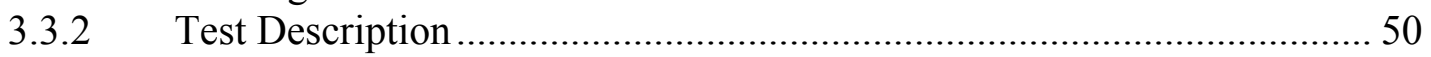

3.3.3 Test Results and Discussion.............................................................. 52

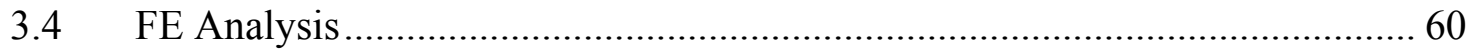

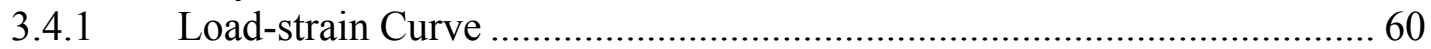

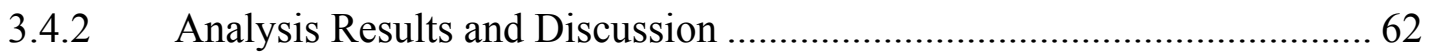

3.5 Determination of the Coefficient of Elastic Restraint...................................... 63

3.6 Comparisons of Test Results with Analytical and FE Predictions .................... 68

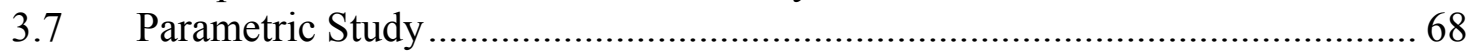

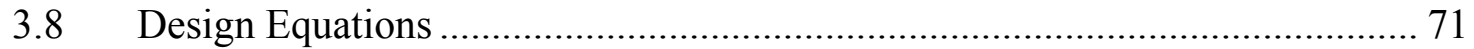

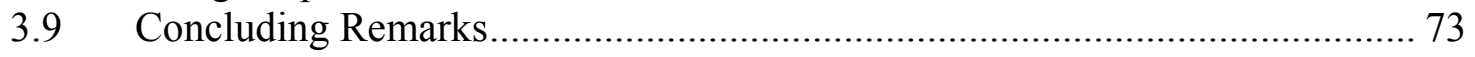

\section{CHAPTER 4 OUT-OF-PLANE SHEAR ….................................................... 76}

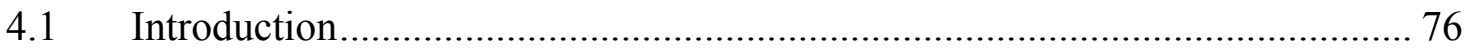

4.2 Analytical Model including Skin Effect ……............................................... 77

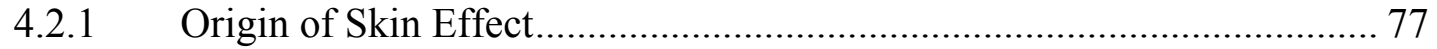

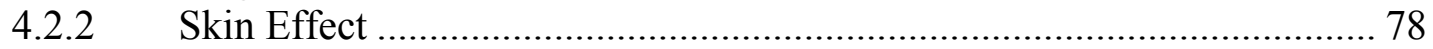

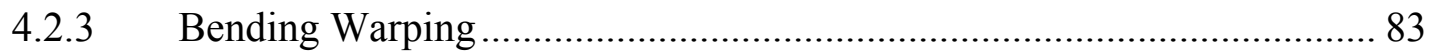

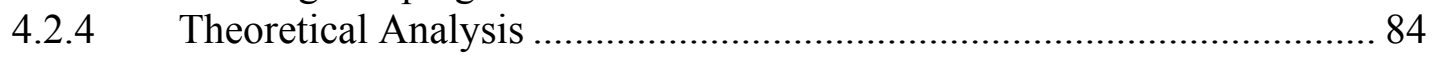

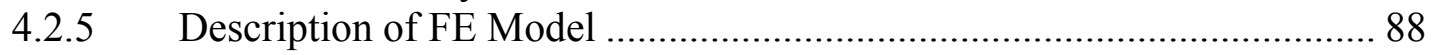

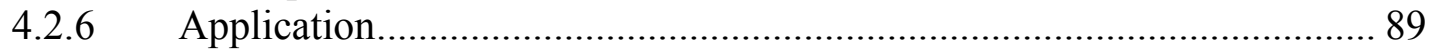

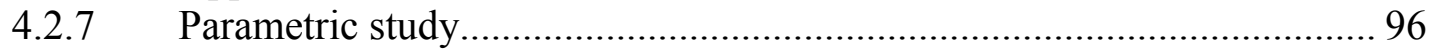

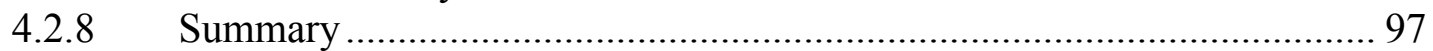

4.3 CER Effect on Shear Stiffness and Interfacial Shear Stress Distribution......... 99

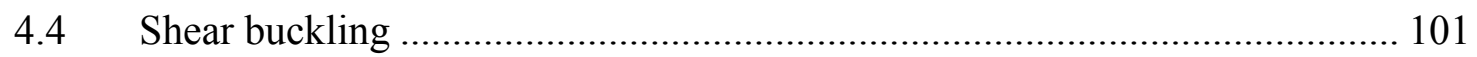

4.5 Proposed Method to Predict Failure Load ................................................... 105

4.5.1 Core-face Delamination................................................................... 105

4.5.2 Core Shear Failure and Shear Buckling................................................ 106

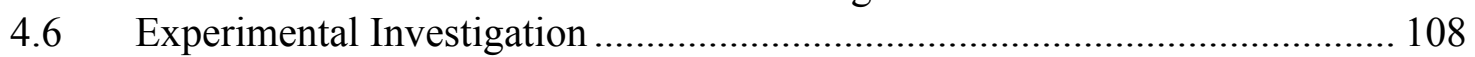

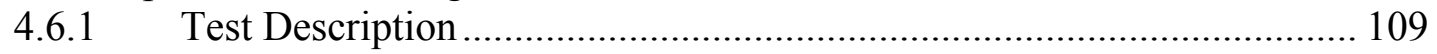

4.6.2 Test Results and Discussion.............................................................. 111

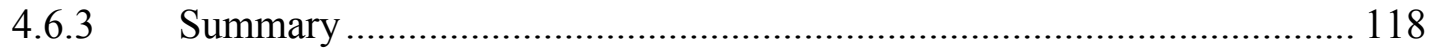

4.7 Correlations between Test Results and Prediction from Design Equations ... 120

4.7.1 Shear failure of Flat Panel............................................................. 120

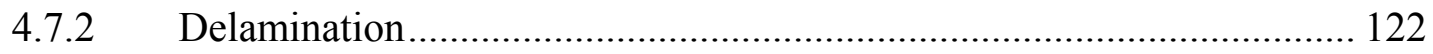

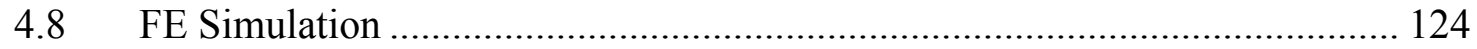

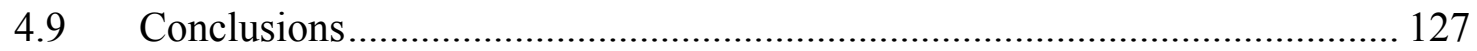




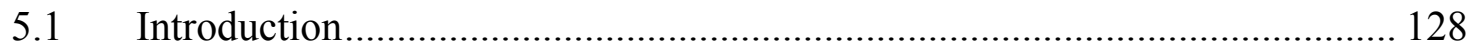

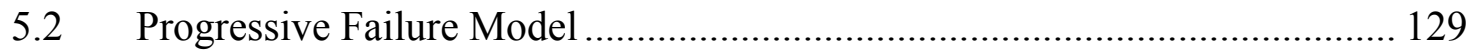

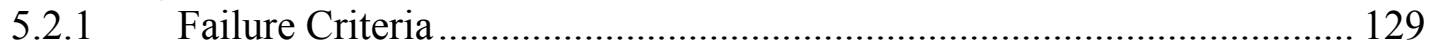

5.2.2 Progressive Failure Analysis............................................................ 131

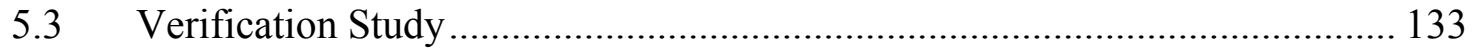

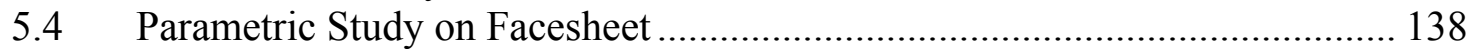

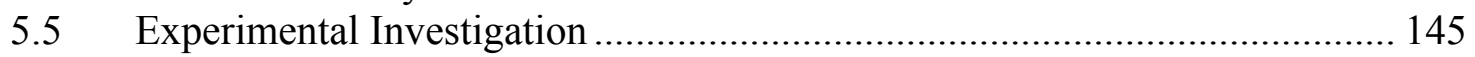

5.5.1 Three-Point Bending Test ............................................................... 146

5.5.2 Compression Test............................................................................ 152

5.6 Correlation between FE and Experimental Results ..................................... 160

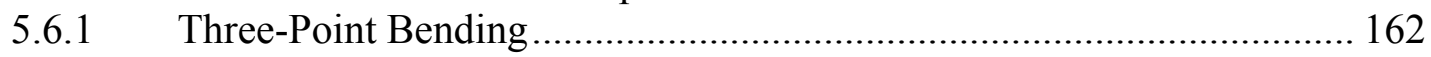

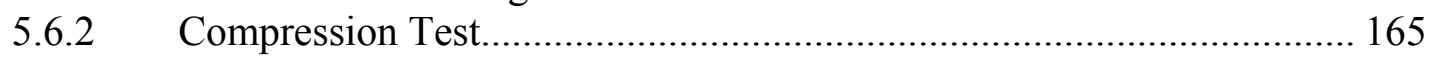

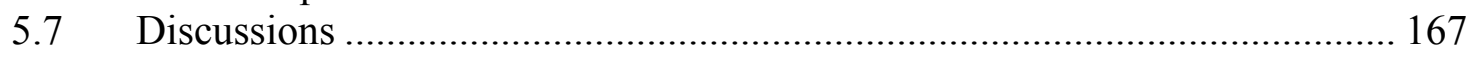

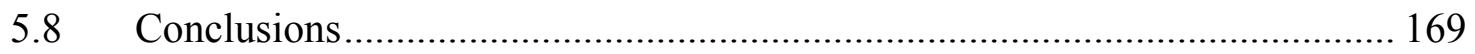

\section{CHAPTER 6 DESIGN GUIDELINES AND RECOMMENDED}

IMPROVEMENTS ........................................................................................................... 177

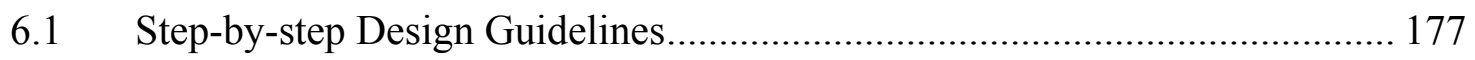

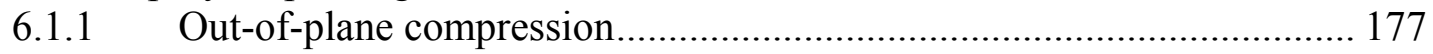

6.1.2 Out-of-plane Shear ......................................................................... 180

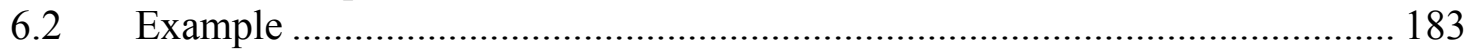

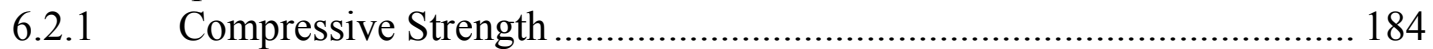

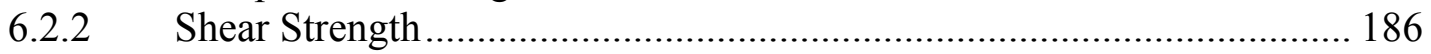

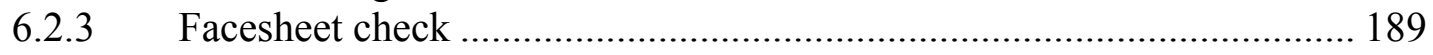

6.3 Recommended Improvements ............................................................... 190

6.3.1 Compression Behavior................................................................... 190

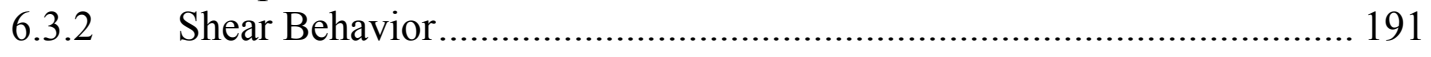

6.3.3 Facesheet Study ............................................................................... 191

CHAPTER 7 CONCLUSIONS AND RECOMMENDATIONS.............................. 193

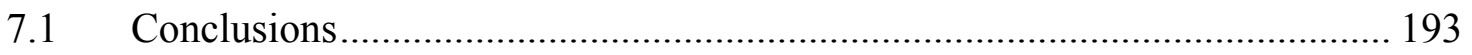

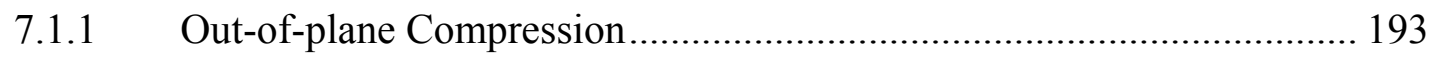

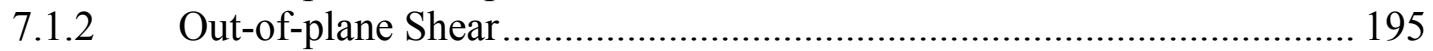

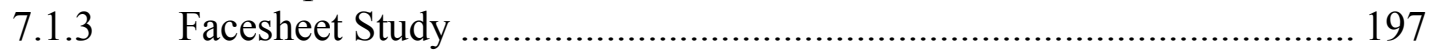

7.1.4 Design Guidelines and Recommended Improvement............................... 198

7.2 Suggestions for Future Work …………………........................................ 198 
PUBLICATIONS .............................................................................................................. 200

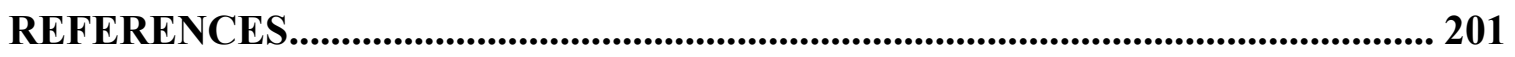

APPENDIX A PROGRAM TO CALCULATE THE CRITICAL BUCKLING

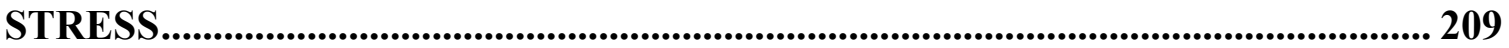

APPENDIX B STRENGTH DATA OF CORE MATERIALS ................................ 213

APPENDIX C DERIVATION OF EQUILIBRIUM EQUATION ........................... 216

APPENDIX D SHEAR TEST FOR FACESHEET LAMINATES ......................... 219

APPENDIX E STIFFNESS OF FACESHEET LAMINATES AND CORE

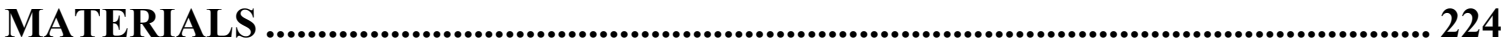




\section{LIST OF FIGURES}

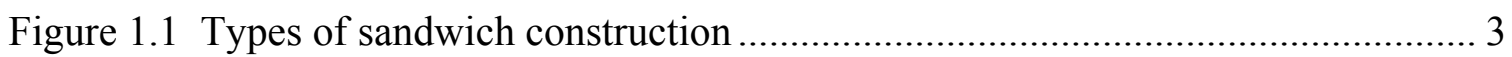

Figure 1.2 HFRP panels with sinusoidal core configuration ......................................... 4

Figure 1.3 FRP decks produced from adhesively bonded .............................................. 6

Figure 1.4 Application of HFRP sandwich panels.................................................... 8

Figure 2.1 Sandwich panel with hexagonal honeycomb core......................................... 23

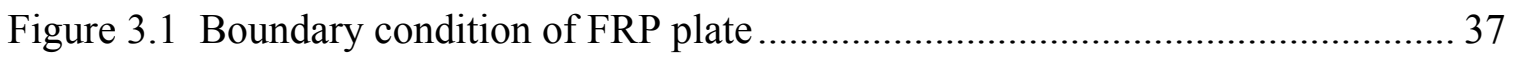

Figure 3.2 In-plane core specimen dimensions........................................................... 44

Figure 3.3 Buckling load vs. elastic restraint coefficient............................................... 45

Figure 3.4 The first buckling mode for clamped condition ............................................ 45

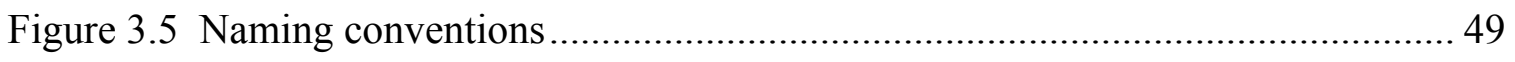

Figure 3.6 Lay-up of facesheet .............................................................................. 51

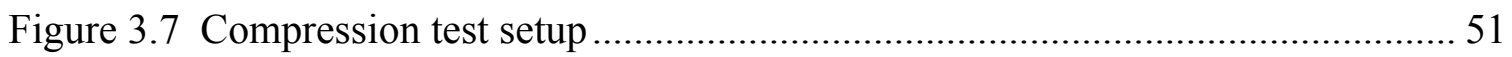

Figure 3.8 Bare compression test specimen.............................................................. 53

Figure 3.9 Failure load for bare compression test.................................................. 54

Figure 3.10 Load-displacement curve for bare compression test .................................... 54

Figure 3.11 Strain-load curve for bare compression test ............................................ 55

Figure 3.12 Stabilized compression test specimen ………......................................... 57

Figure 3.13 Load-displacement curve for stabilized compression test............................ 57

Figure 3.14 Strain-load curve for stabilized compression test........................................ 58

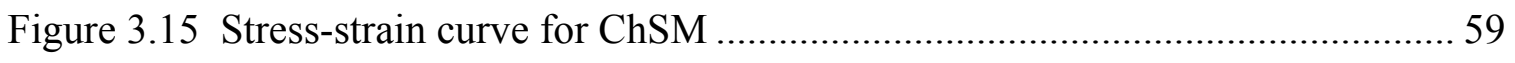

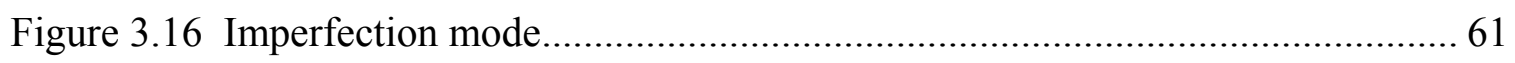

Figure 3.17 Load-strain curves for stabilized compression test ..................................... 61

Figure 3.18 Load-strain curves for bare compression test............................................. 62

Figure 3.19 Test setup to determine the elastic restraint coefficient .............................. 63

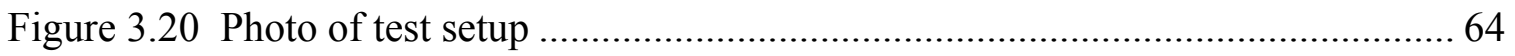

Figure 3.21 Critical buckling stress vs. core height..................................................... 70

Figure 3.22 Critical buckling stress vs. length of flat panel ......................................... 70

Figure 3.23 Buckling load vs. core height .............................................................. 71

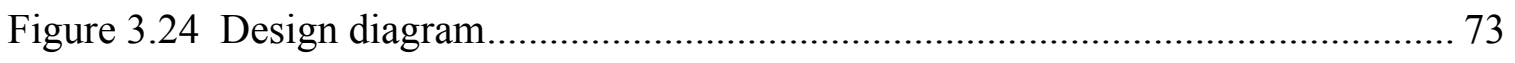

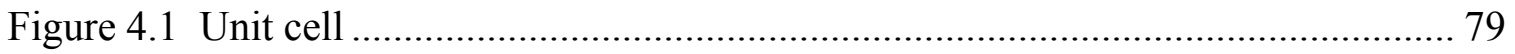




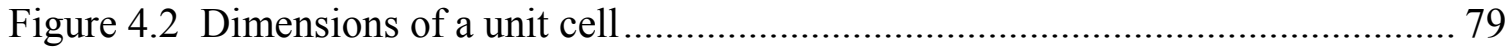

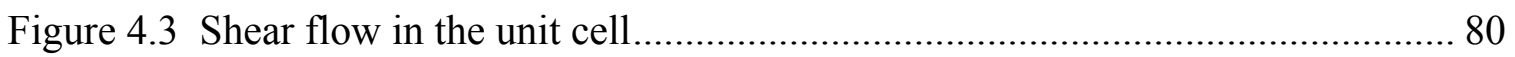

Figure 4.4 Shear flow in the RVE....................................................................... 80

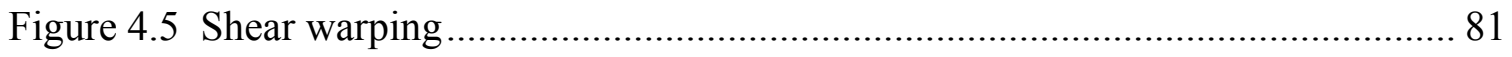

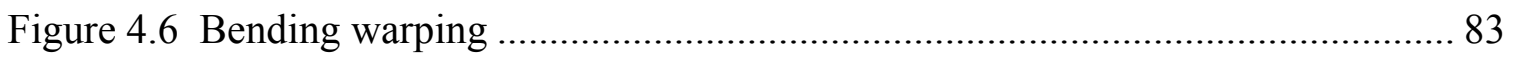

Figure 4.7 Model used for theoretical analysis ........................................................ 84

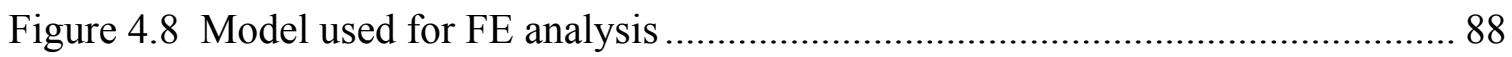

Figure 4.9 Stiffness vs. aspect ratio ..................................................................... 90

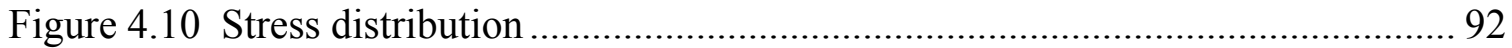

Figure 4.11 Comparisons between FE and analytical result for stress distribution (along length)

Figure 4.12 Comparisons between FE and analytical result for stress distribution (along

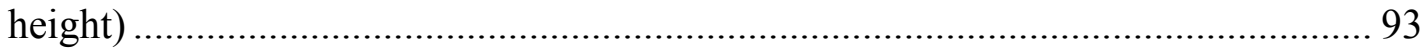

Figure 4.13 Stress distribution with bending warping based on FE analysis ................ 94

Figure 4.14 Normal interfacial stresses vs. height.................................................... 96

Figure 4.15 CER effect on transverse shear stiffness .............................................. 99

Figure 4.16 CER effect on interfacial shear stress................................................... 99

Figure 4.17 Boundary condition of FRP plate ...................................................... 101

Figure 4.18 Critical shear stress vs. aspect ratio for one bonding layer ...................... 103

Figure 4.19 Critical shear stress vs. aspect ratio for different bonding layers.............. 103

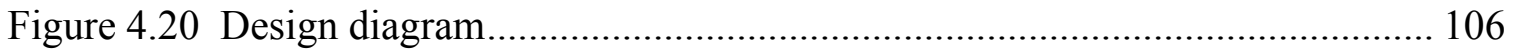

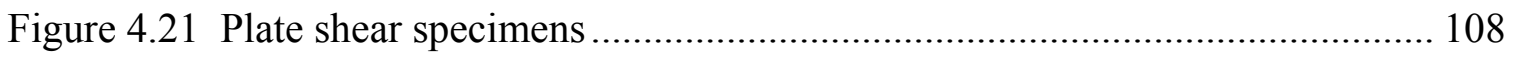

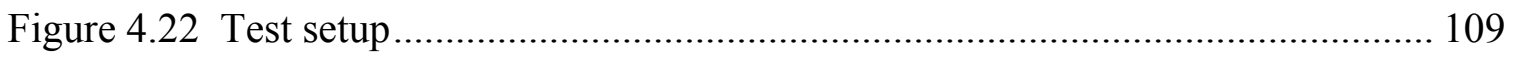

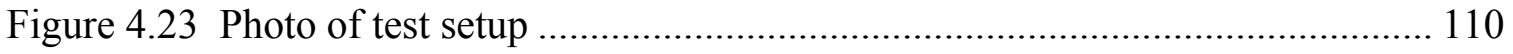

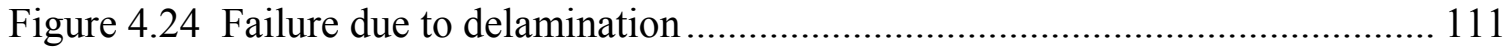

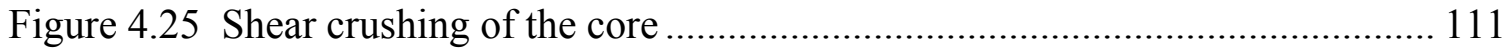

Figure 4.26 Load-displacement curve for longitudinal test ...................................... 113

Figure 4.27 Load-strain curve for longitudinal test .............................................. 114

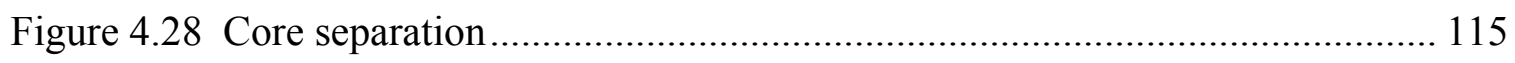

Figure 4.29 Load-displacement curve for transverse test ...................................... 116

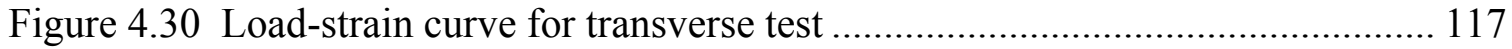

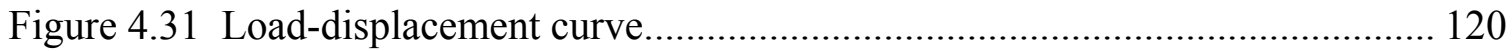


Figure 4.32 Finite element model of the four-point bending test of an HFRP sandwich panel with sinusoidal wave core configuration (from Wang, 2004)....................... 126

Figure 4.33 Finite element results compared to experimental data of the four-point bending test (from Wang, 2004)..................................................................... 126

Figure 5.1 Load-displacement paths .................................................................. 136

Figure 5.2 Comparison of ultimate load and displacement ........................................ 137

Figure 5.3 Load-displacement curve for \#3 ............................................................ 141

Figure 5.4 Load-displacement curve for \#7 ………………..................................... 142

Figure 5.5 Load-displacement curves for CL ....................................................... 143

Figure 5.6 Normalized failure strength................................................................. 144

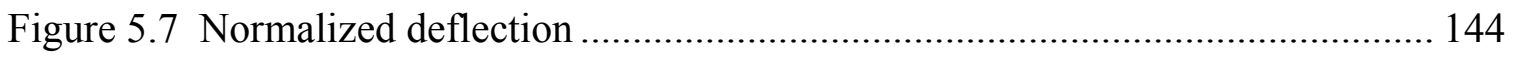

Figure 5.8 Three-point bending test setup ............................................................... 147

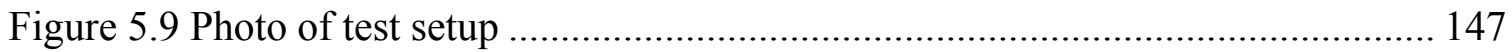

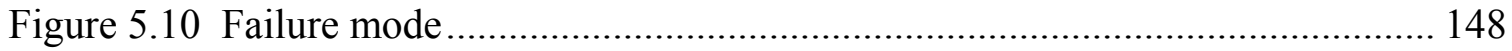

Figure 5.11 Load vs. displacement ..................................................................... 150

Figure 5.12 Load vs. strain .............................................................................. 151

Figure 5.13 Experimental setup for compression test................................................ 154

Figure 5.14 Close shot of compression fixture ........................................................... 155

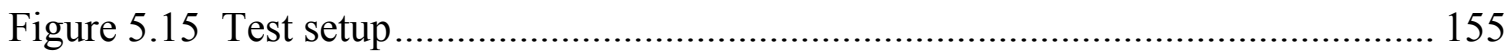

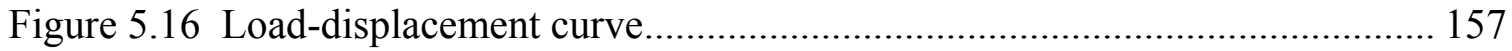

Figure 5.17 Load-strain curve............................................................................ 158

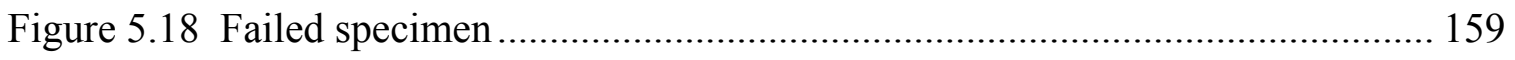

Figure 5.19 Failure mode comparison ................................................................ 159

Figure 5.20 Comparison of failure load..................................................................... 163

Figure 5.21 Load-displacement paths for Laminate 3 ………………….................... 163

Figure 5.22 Load-strain paths for Laminate 3 ............................................................ 164

Figure 5.23 Comparison of failure load................................................................. 166

Figure 5.24 Load-displacement paths for Laminate 3 ……….................................. 166

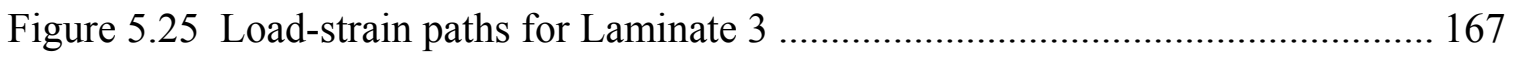

Figure 5.26 Normalized deflection under patch load................................................... 168

Figure 5.27 Normalized strength .............................................................................. 169

Figure 5.28 Load-displacement paths for Laminate 1L under bending ......................... 171

Figure 5.29 Load-strain paths for Laminate 1L under bending ..................................... 171 
Figure 5.30 Load-displacement paths for Laminate 1T under bending ...................... 172

Figure 5.31 Load-strain paths for Laminate $1 \mathrm{~T}$ under bending ................................. 172

Figure 5.32 Load-displacement paths for Laminate 2 under bending ......................... 173

Figure 5.33 Load-strain paths for Laminate 2 under bending ................................. 173

Figure 5.34 Load-displacement paths for Laminate 1L under compression ................ 174

Figure 5.35 Load-strain paths for Laminate 1L under compression............................ 174

Figure 5.36 Load-displacement paths for Laminate 1T under compression ................ 175

Figure 5.37 Load-strain paths for Laminate 1T under compression........................... 175

Figure 5.38 Load-displacement paths for Laminate 2 under compression ................... 176

Figure 5.39 Load-strain paths for Laminate 2 under compression ............................. 176

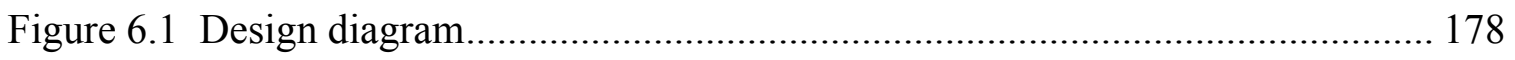

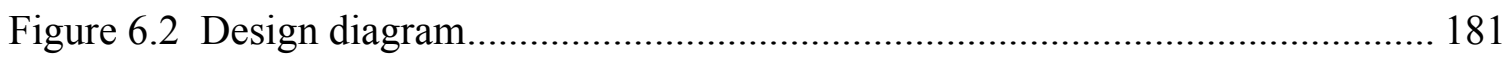

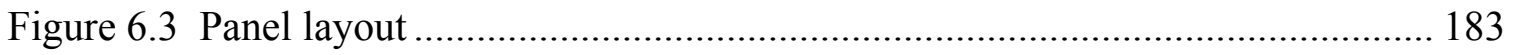

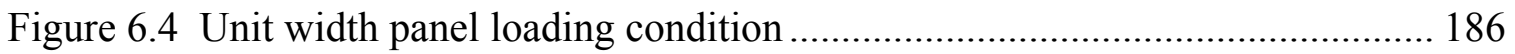

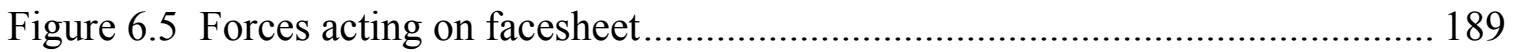

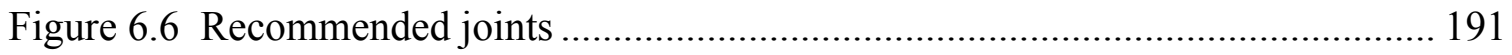




\section{LIST OF TABLES}

Table 1.1 Summary of deck characteristic for two fabrication methods ......................... 7

Table 3.1 Properties of the core material ...................................................................... 44

Table 3.2 Comparison between FE and analytical result........................................... 47

Table 3.3 Comparison between FE and analytical result for multi-cell panel................ 47

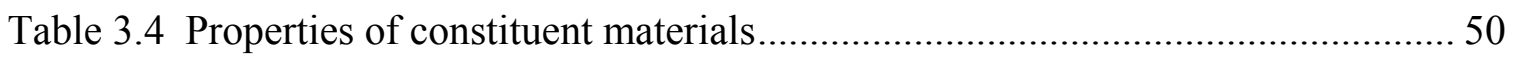

Table 3.5 Layer properties of face laminate and core materials ................................... 50

Table 3.6 Average value and standard deviation of failure load for bare compression

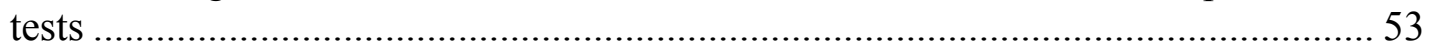

Table 3.7 Average value of failure load for stabilized compression test........................ 56

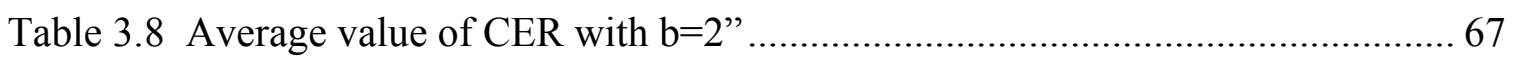

Table 3.9 Comparison of analytical, FE and test results for buckling load .................... 68

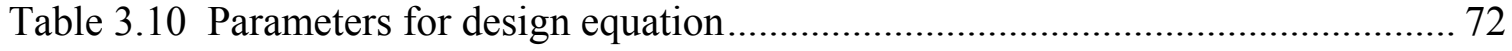

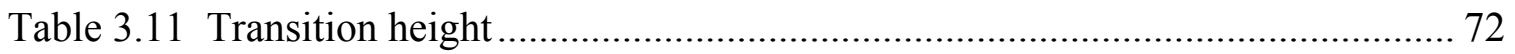

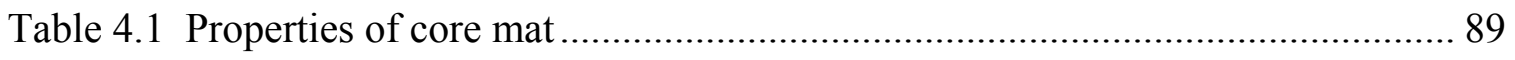

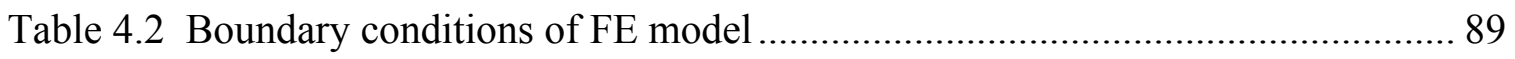

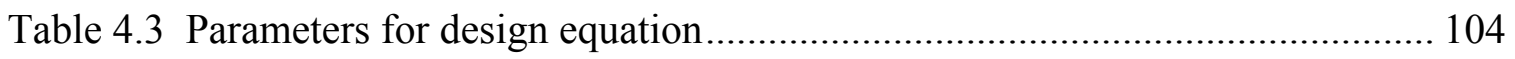

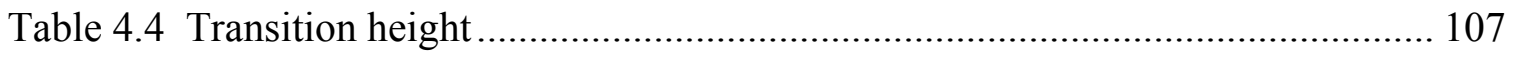

Table 4.5 Average value of failure load for longitudinal samples............................. 112

Table 4.6 Average value of failure load for transverse samples.................................. 118

Table 4.7 Parameters for sandwich beam specimen with excessive bonding layers .... 120

Table 4.8 Interfacial tensile strength (from Wang, 2004) ........................................ 123

Table 4.9 Nominal interfacial tensile strength ........................................................ 124

Table 4.10 Fracture toughness and interfacial strength for the four-point bending test. 124

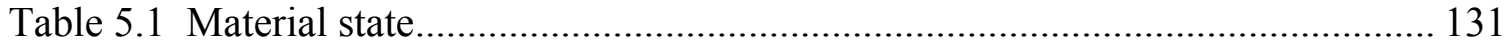

Table 5.2 Material properties and strength parameters.......................................... 134

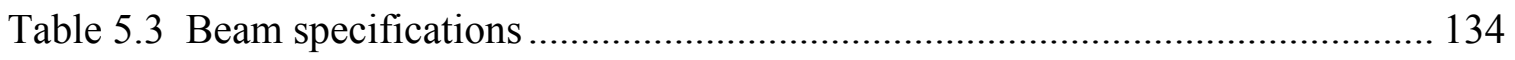

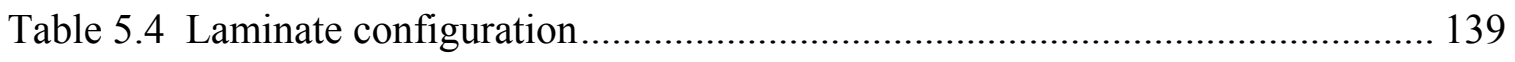

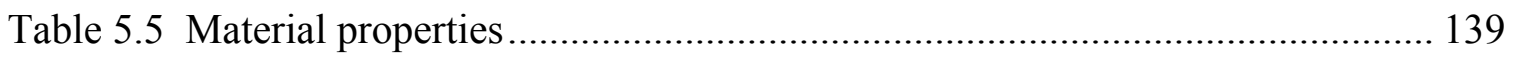

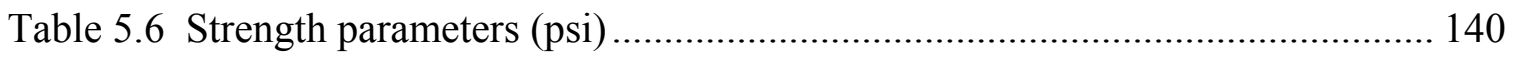

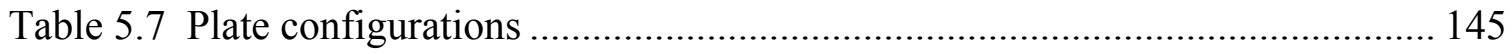


Table 5.8 Experimental results for bending test ................................................. 148

Table 5.9 Experimental results for compression test ............................................... 156

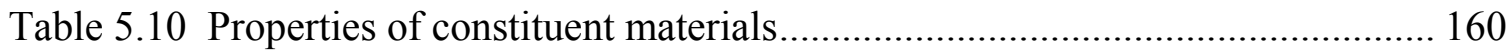

Table 5.11 Layer properties of face laminates ...................................................... 160

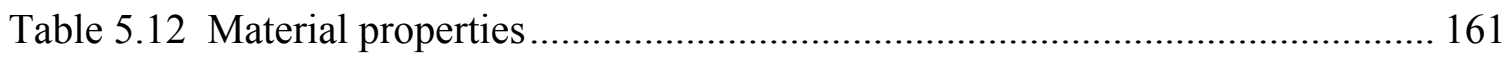

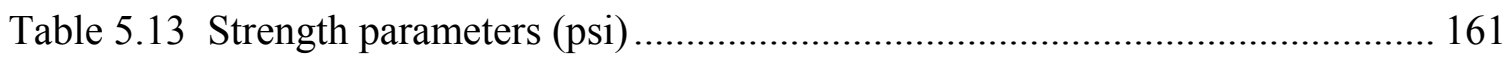

Table 5.14 Comparison of failure load for three-point bending test........................... 162

Table 5.15 Comparison of failure load for compression test..................................... 165

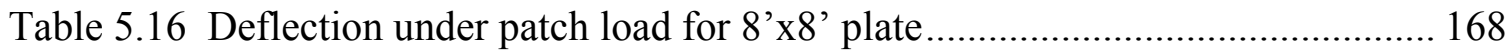

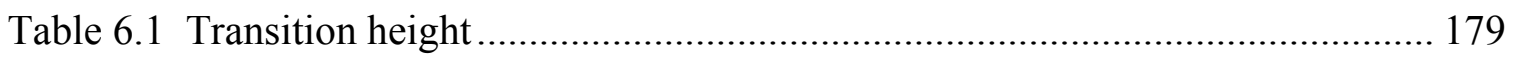

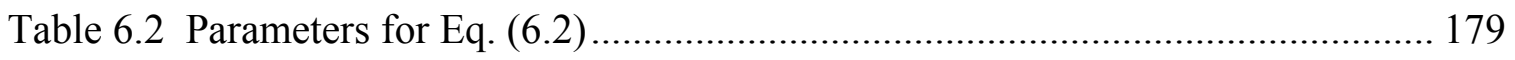

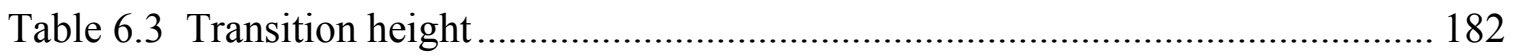

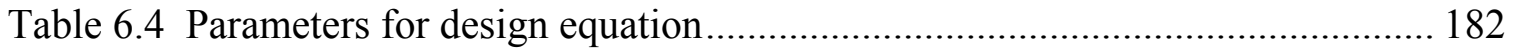

Table 6.5 Nominal interfacial tensile strength (psi)............................................... 182

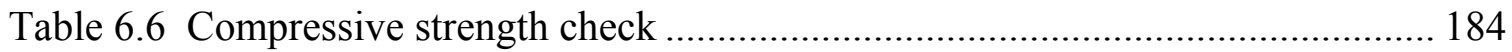

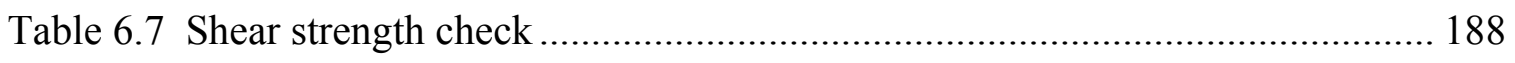

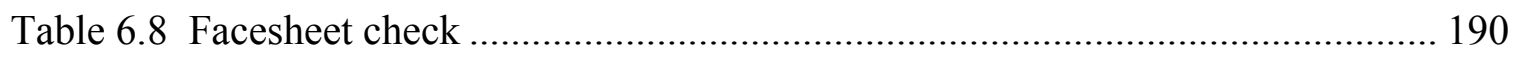

Table 6.9 Comparison between current and recommended configuration ................... 190 


\section{ABBREVIATIONS}

\begin{tabular}{|c|c|c|}
\hline \multicolumn{2}{|c|}{ AASHTO } & $=\quad$ American Association of State Highway and Transportation \\
\hline AST & $=$ & $\begin{array}{l}\text { Otticial } \\
\text { Advanced Sandwich Theory }\end{array}$ \\
\hline ASTM & & American Society for Testing and Materials \\
\hline $\mathrm{Bi}$ & $=$ & Bi-directional lamina \\
\hline BLCS & $=$ & Beam element with Layer-wise Constant Shear \\
\hline CBT & $=$ & Composite Beam Theory \\
\hline CER & $=$ & Coefficient of Elastic Restraint \\
\hline ChSM & $=$ & Chopped Strand Mat \\
\hline $\mathrm{CL}$ & $=$ & Compression along Longitudinal direction \\
\hline CT & $=$ & Compression along Transverse direction \\
\hline CZM & $=$ & Cohesive Zone Model \\
\hline EST & $=$ & Elementary Sandwich Theory \\
\hline FE & $=$ & Finite Element \\
\hline FPF & $=$ & First-ply-failure \\
\hline FRP & $=$ & Fiber-Reinforced Polymer \\
\hline FHWA & & $=\quad$ Federal Highway Administration \\
\hline FWT & $=$ & Flatwise Tension Test \\
\hline HFRP & $=$ & Honeycomb Fiber-Reinforced Polymer \\
\hline KSCI & $=$ & Kansas Structural Composites, Inc. \\
\hline LVDT: & & Linear Variable Differential Transformer \\
\hline MPC & $=$ & Multiple Point Restraint \\
\hline MTS & $=$ & Mechanical Testing System \\
\hline RVE & $=$ & Representative Volume Element \\
\hline TL & $=$ & Tension along Longitudinal direction \\
\hline TT & $=$ & Tension along Transverse direction \\
\hline Uni & $=$ & Unidirectional lamina \\
\hline
\end{tabular}




\section{NOTATIONS}

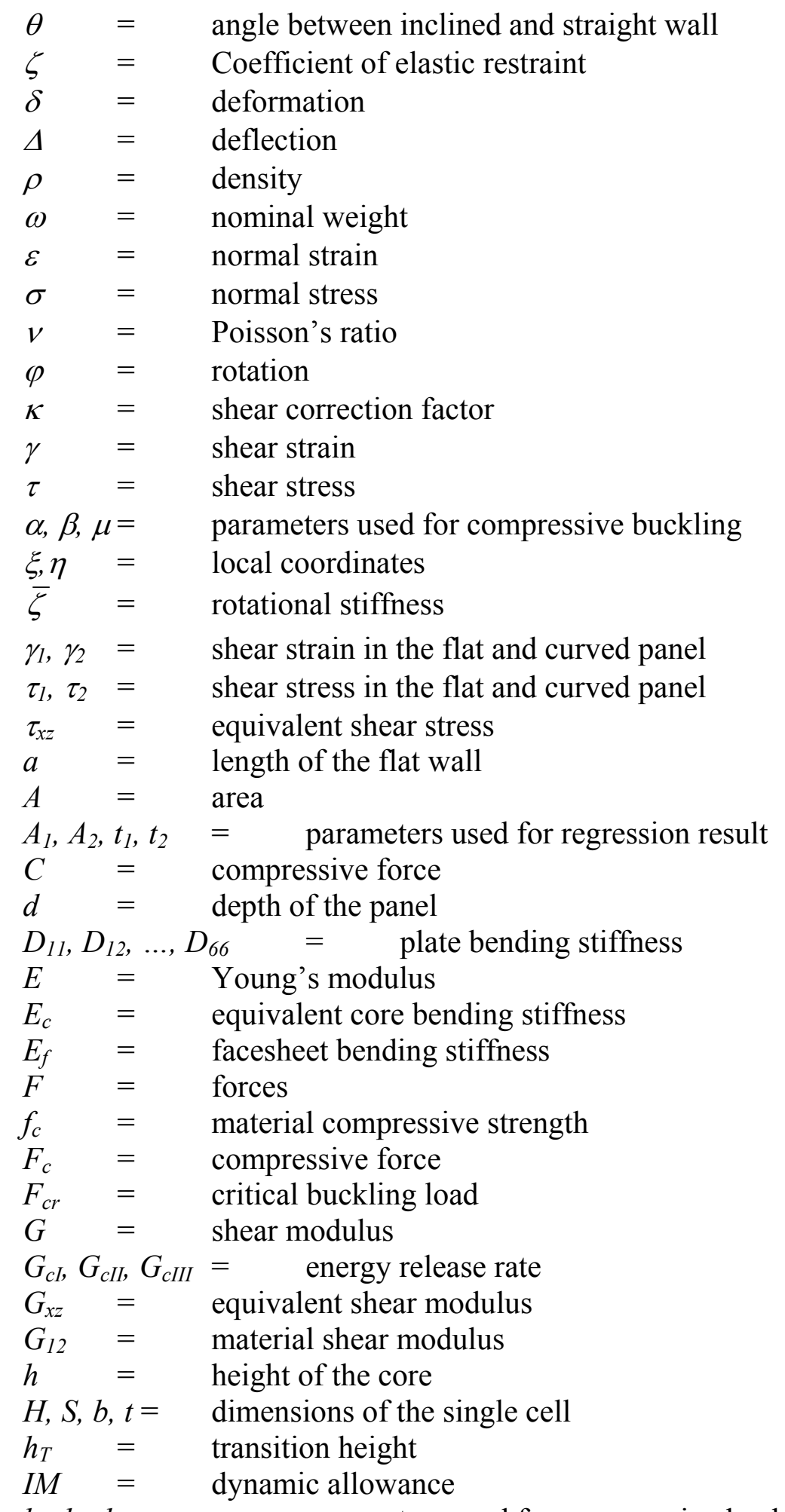

$k_{1}, k_{2}, k_{3}=$ parameters used for compressive buckling 


$\begin{array}{llll}l & = & \text { curved panel length } \\ L & = & \text { span length } \\ M & = & \text { moment } \\ N_{y}, N_{x y}= & \text { critical buckling stress } \\ P & = & \text { patch load } \\ p, q= & \text { parameters used for simplified equation to predict compressive buckling } \\ q & = & \text { load } \\ R & = & \text { distribution load } \\ S & = & \text { distance between loading points } \\ S_{22} & = & \text { interfacial normal stress } \\ S(\xi) & = & \text { curve length } \\ T & = & \text { tensile force } \\ t & = & \text { thickness of the core wall } \\ t_{f} & = & \text { facesheet thickness } \\ U & = & \text { total strain energy } \\ u, v & = & \text { displacement } \\ U_{a} & = & \text { strain energy related to axial response } \\ U_{b} & = & \text { strain energy related to bending } \\ U_{s} & = & \text { strain energy related to shear } \\ V & = & \text { shear force } \\ v(x, y) & = & \text { displacement } \\ V_{f}= & \text { fiber volume fraction } \\ W & = & \text { work done by external force } \\ w(x, y) & = & \text { shape function } \\ x, y, z= & \text { global coordinates } \\ X_{t}, X_{c}, Y_{T}, Y_{C}, & S_{12}, S_{23}, S_{D S} \quad \\ Y & = & \text { load factor } \\ & & \end{array}$




\section{CHAPTER 1}

\section{INTRODUCTION}

\subsection{Problem Statement and Research Significance}

\subsubsection{Development of Sandwich Structures}

A typical sandwich panel is made of two stiff skins, separated by a lightweight honeycomb core. In general, cores fall into four types, as shown in Fig. 1.1: (a) foam or solid core, (b) honeycomb core, (c) web core, and (d) corrugated or truss core (Vinson, 1999). Sandwich construction is playing an increasingly important role in structure because of its exceptionally high flexure stiffness-to-weight ratio compared to monocoque and other architectures. As a result, sandwich construction results in lower lateral deformation, higher buckling resistance, and higher natural frequencies than other constructions. Thus, for a given set of mechanical and environmental loads, sandwich construction often results in a lower structural weight than other configurations. It may be designed so that each component is utilized to its ultimate limit. This feature makes it to be attractive in various engineering fields where stiffness and strength must be met with minimum weight. 
Sandwich construction is relatively new, even though Noor et al. (1996) reported that the concept of sandwich construction has been traced back to the mid $19^{\text {th }}$ century (Fairbairn, 1849). It has been widely used primarily in the aircraft industry since the 1940's, with the development of the British Mosquito bomber, and later logically extended to missile and spacecraft structures. Bitzer (1992) gave an excellent review of the uses of honeycomb core materials and applications. He pointed out that every two engine aircrafts in the west utilizes some honeycomb sandwich structure. Nowadays sandwich panels and shells have been widely used in aerospace, shipbuilding, civil infrastructures and other industries. Some usages include railcar, racing boats, auto racing cars, snow skis, water skis, kayaks, canoes, pool tables, platform tennis paddles, etc. In recent years, the demands in civil infrastructure have provided opportunities for development and implementation of Honeycomb Fiber-Reinforced Polymer (HFRP) sandwich panels, both in rehabilitation projects and new constructions. Among others, the concept of lightweight and heavy duty HFRP panels, with sinusoidal core configuration in the plane extending vertically between face laminates, was introduced for highway bridge decks by Plunkett (1997) (see Fig. 1.2). This product was successfully implemented in several bridge projects (Davalos et al., 2001). 


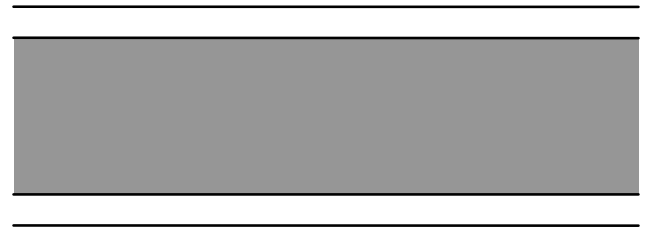

(a) Foam Core Sandwich

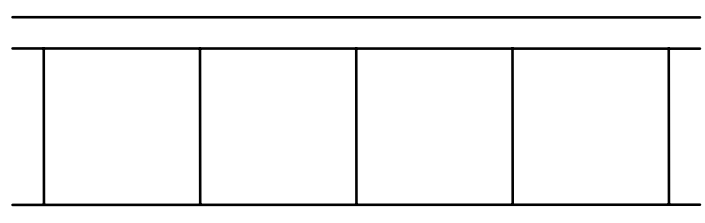

(b) Honeycomb Core Sandwich

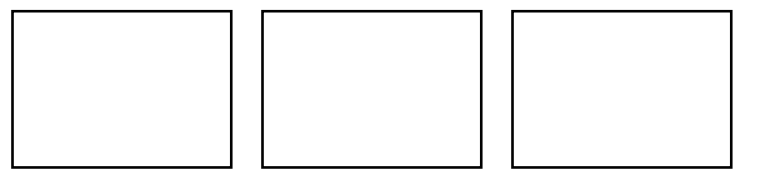

(c) Web Core Sandwich

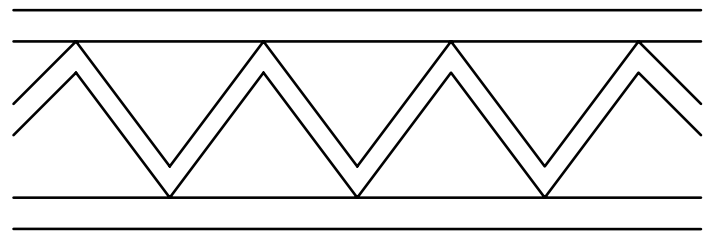

(d) Truss Core Sandwich

Figure 1.1 Types of sandwich construction 


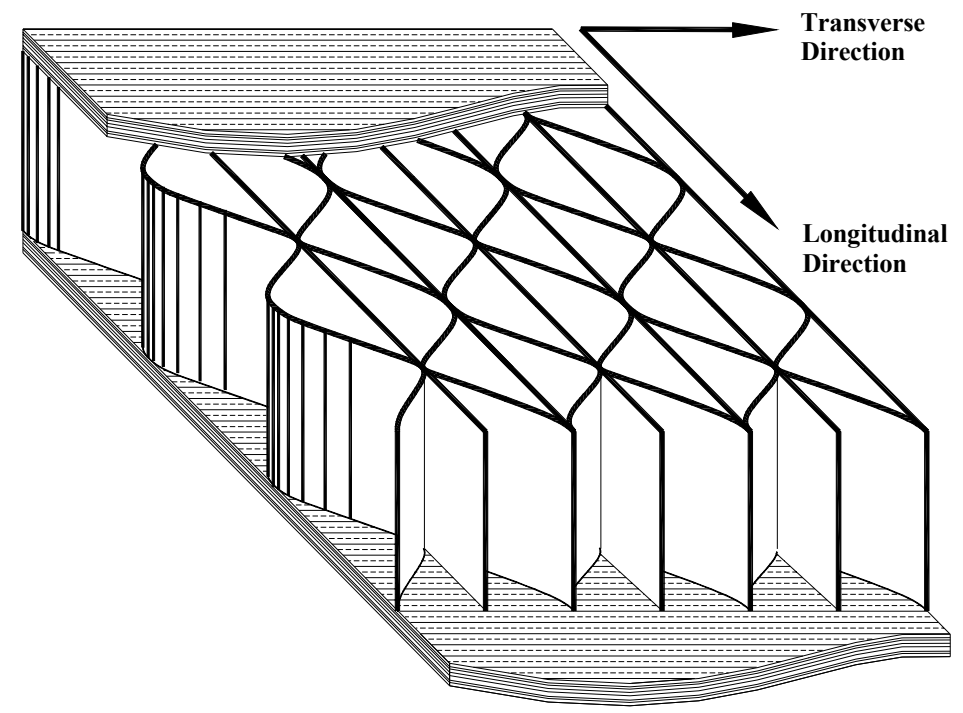

Figure 1.2 HFRP panels with sinusoidal core configuration

\subsubsection{Application in Civil Infrastructure}

According to a report from Market Development Alliance of the FRP Composites Industry (MDA, 2003), today's bridge owners are faced with unique challenges as a result of a severely deteriorating infrastructure, insufficient funding and a demanding public. A recent released study (Report FHWA-RD-01-156) funded by FHWA estimates the annual direct cost of corrosion for highway bridges to be $\$ 6.43$ billion to $\$ 10.15$ billion. This includes $\$ 3.79$ billion to replace structurally deficient bridges over next 10 years and $\$ 1.07$ billion to $\$ 2.93$ billion for maintenance and cost of capital for concrete bridge decks. In addition to these direct costs, the study's life-cycle analysis estimates indirect costs to the user due to traffic delays and lost productivity at more than 10 times the direct cost of corrosion. Although most bridge owners continue to make decisions based on lower initial cost, it has become extremely clear that this approach does not 
work and in the near future more money will be spent maintaining existing structures than building new ones. As a result, there are tremendous opportunities for FRP bridge decks that are corrosion resistant, and can be rapidly installed.

FRP bridge decks have only been used in the United States since the mid-1990's. Primary benefits of FRP decks include: durability, lightweight, high strength, rapid installation, lower or competitive life-cycle cost, and high quality manufacturing processes under controlled environments. Compared with cast-in-place concrete decks, FRP bridge decks typically weigh $80 \%$ less, can be erected twice as fast and have service lives that can be two to three times greater. Although based on initial in-place material cost, FRP bridge decks typically cost 2-3 times that a conventional deck, life-cycle costs, light weight and rapid installation tend to be features that justify the use of FRP bridge decks.

FRP bridge decks commercially available at the present time can be classified according to two types of construction: sandwich and adhesively bonded pultruded shapes. For sandwich constructions, cellular materials are the most efficient core materials for weight-sensitive applications. Due to the ease with which facesheets and core materials can be changed in manufacturing, sandwich construction presents tremendous flexibility in designing for varied depths and deflection requirements. Facesheets of sandwich bridge decks are primarily composed of E-glass mat and/or roving infused with polyester or vinylester resins. Current core materials are rigid foams of thin-walled cellular FRP materials, such as shown in Fig. 1.2. 
(a)

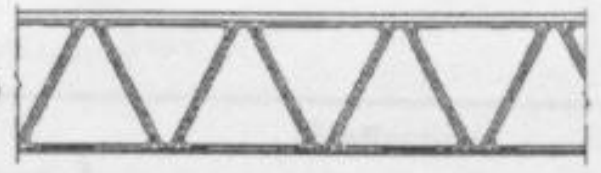

(b)

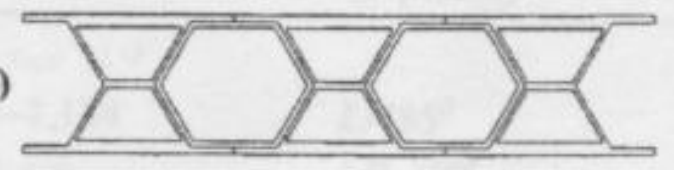

(c)

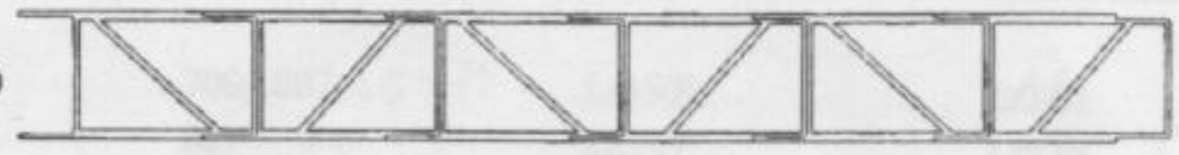

(d)

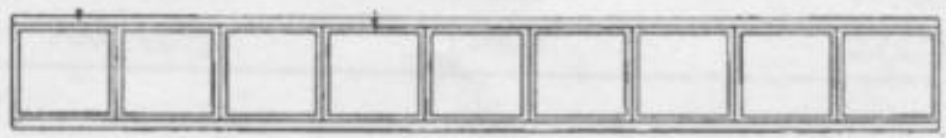

Figure 1.3 FRP decks produced from adhesively bonded pultruded shapes: (a) EZSpan (Atlantic Research); (b) Superdeck (Creative Pultrusions); (c) DuraSpan (Martin Marietta Materials); (d) square tube and plate deck (Strongwell)

(from Bakis et al., 2002)

Most currently available commercial decks are constructed using assemblies of adhesively bonded pultruded shapes. Such shapes can be economically produced in continuous lengths by numerous manufacturers using well-established processing methods. Design flexibility on this type of deck is obtained by changing the constituents of the shapes (such as fibers and fiber orientations) and, to a lesser extent. Several decks constructed with pultruded shapes are shown in Fig. 1.3. The pultruded shapes are typically aligned transverse to the traffic direction. Each deck design has advantages in terms of stiffness, strength and field implementation. In laboratory testing, the observed failures in such decks are generally by local punching shear and crushing or large-scale delamination of the shapes constituting the cross section. 
Table 1.1 Summary of deck characteristic for two fabrication methods

(from Bakis et al., 2002)

\begin{tabular}{|l|l|l|l|l|l|}
\hline Deck System & $\begin{array}{l}\text { Depth } \\
\text { (in.) }\end{array}$ & $\mathrm{lb} / \mathrm{ft}^{2}$ & Dollar/ $\mathrm{ft}^{2}$ & $\begin{array}{l}\text { Deflection } \\
\text { (reported) }\end{array}$ & $\begin{array}{l}\text { Deflection } \\
\text { (normalized) }\end{array}$ \\
\hline $\begin{array}{l}\text { Sandwich Construction } \\
\text { Hardcore Composites }\end{array}$ & $7-28$ & $20-23$ & $53-110$ & $\mathrm{~L} / 785$ & $\mathrm{~L} / 1,120$ \\
KSCI & $5-24$ & 15 & 65 & $\mathrm{~L} / 1,300$ & $\mathrm{~L} / 1,300$ \\
\hline Adhesively Bonded Pultrusions & & & & & \\
DuraSpan & 7.5 & 18 & $65-75$ & $\mathrm{~L} / 450$ & $\mathrm{~L} / 340$ \\
SuperDeck & 8 & 22 & 75 & $\mathrm{~L} / 530$ & $\mathrm{~L} / 530$ \\
EZSpan & 9 & 20 & $80-100$ & $\mathrm{~L} / 950$ & $\mathrm{~L} / 950$ \\
Strongwell & $5-8$ & 23 & 65 & $\mathrm{~L} / 605$ & $\mathrm{~L} / 325$ \\
\hline
\end{tabular}

A technical comparison of sandwich and pultruded decks is shown in Table 1.1, from which we can see that the sandwich panels provided by KSCI excel in terms of weight, cost, and deflection among all commercial FRP decks. In addition, the flexibility of the manufacturing process permits custom production of panels of any depth, while a pultruded section has a fixed geometry dictated by the forming steel die used. It is not surprising to find that more than ten bridges have already been built with this novel sandwich panels in the US, which is the focus of this study. A particular example (West Buckeye Bridge, Monongalia County, WV) is shown in Fig. 1.4. 


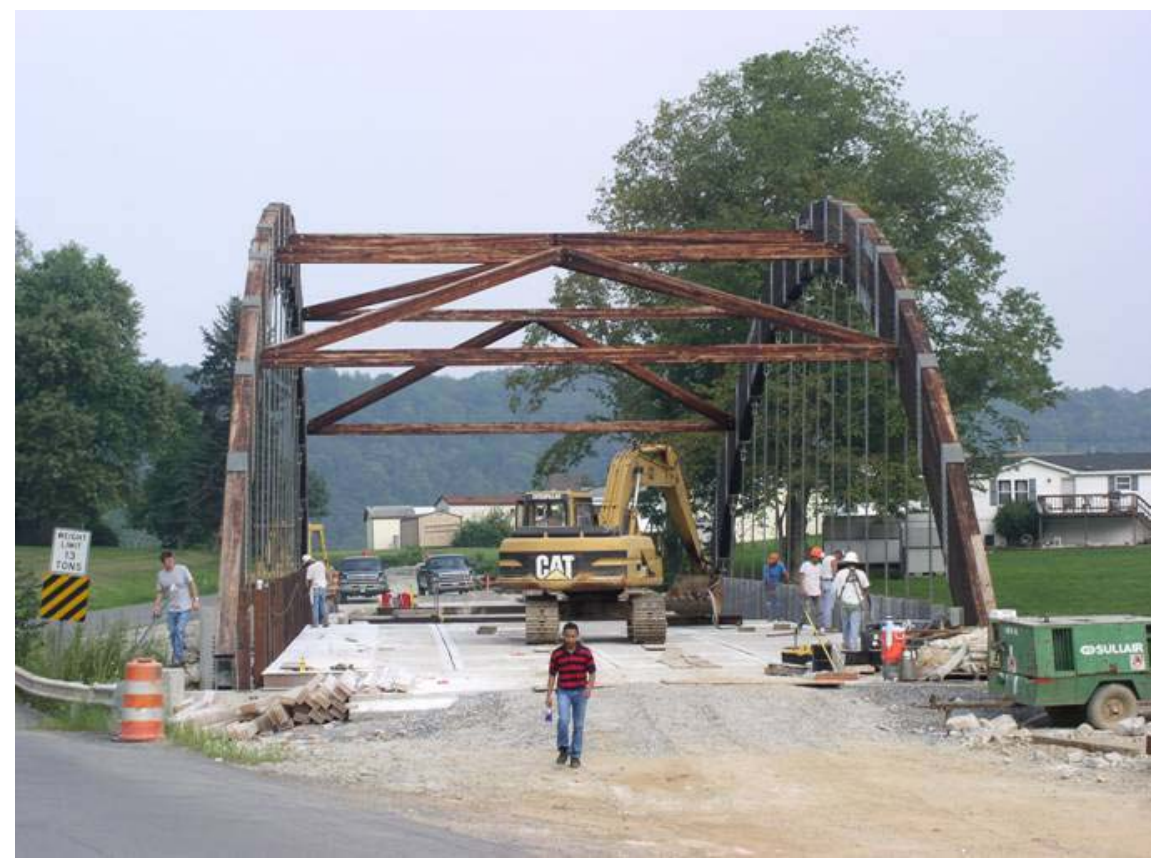

(a) West Buckeye bridge

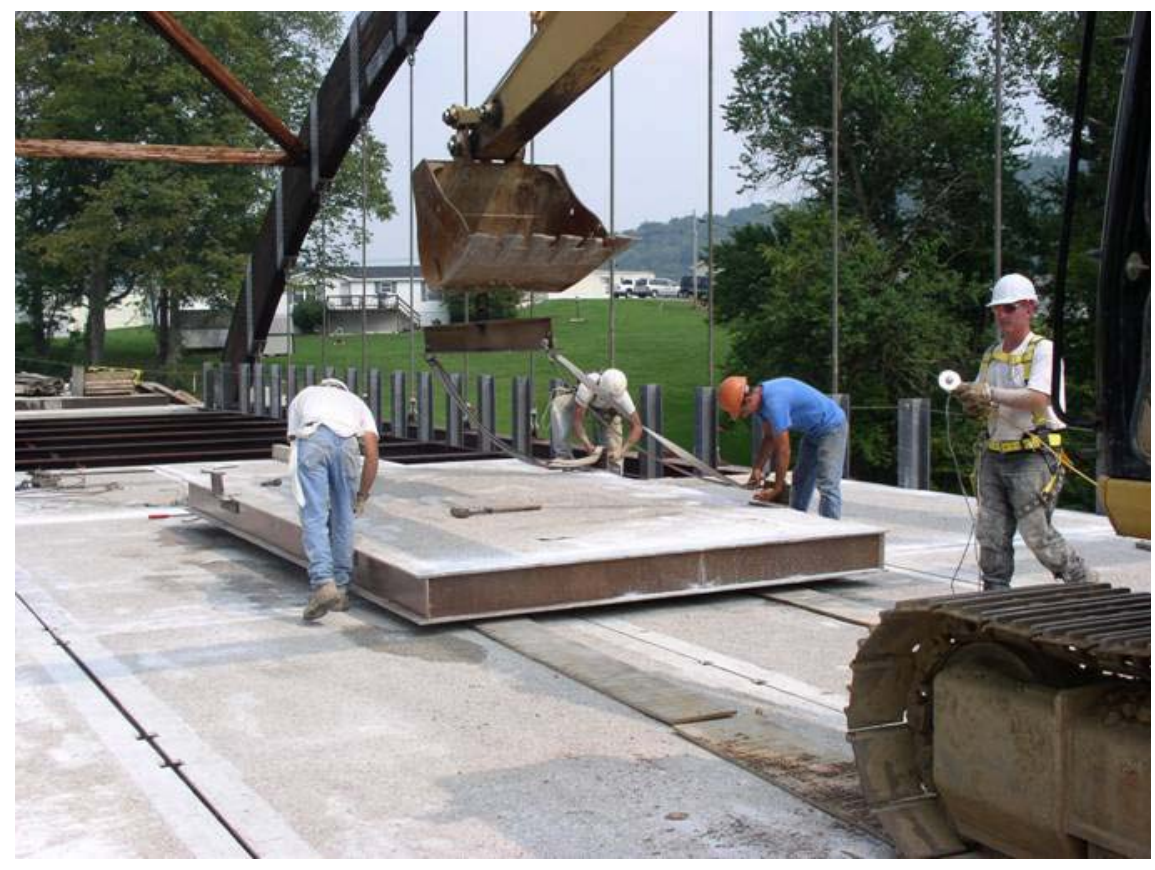

(b) Bridge decks assembling

Figure 1.4 Application of HFRP sandwich panels 


\subsubsection{Research Significance}

Thus far, the design process for sandwich decks is not in a code format. Rather, individual decks are designed on a job-by-job basis using FE techniques. The development of standards and guidelines is needed in order to promote wider acceptance of composite sandwich products in construction. Characterizations of stiffness and strength properties are necessary to facilitate the development of design guidelines. Much effort has been devoted to the modeling and optimization of the HFRP panel shown in Fig. 1.2. Davalos et al. (2001) developed equivalent orthotropic properties representative of the complex honeycomb geometry. Equivalent properties of face laminate are obtained using micro/mechanics models, while the effective orthotropic properties of honeycomb core are obtained from a homogenization process using combined energy method and mechanics of materials approach. It is noted that in the derivation of the transverse shear stiffness, the presence of face laminate is neglected. Thus, only lower bound was provided. They also presented a simplified analysis procedure that can be used in design applications. However, there is still no study available on the strength properties of this HFRP sandwich structure, partly due to the complicated honeycomb core geometry. Therefore, there is a need to further characterize the strength of this product and accurately describe the transverse shear stiffness, which is the objective of this study.

\subsection{Objective and Scope}

This study is focused on strength evaluation of Honeycomb Fiber-Reinforced Polymer (HFPR) sandwich panels with sinusoidal core geometry, both in terms of 
experimental investigation and theoretical analysis. The sandwich structures consist of core and facesheet, where core materials are primarily subjected to out-of-plane compression and shear, and the facesheet carries mainly membrane forces. Therefore, the first objective is to study the core material under out-of-plane compression. Chopped Strand Mat (ChSM) is used for the core material, which is composed of E-glass fibers and polyester resin. The facesheet is made of several layers of ChSM, $0^{\circ} / 90^{\circ}$ E-glass fiber and polyester resins. The ChSM material is used at the interface between core and facesheet as a bonding layer. These component parts are joined by the contact molding manufacturing process. As a result, the number of the ChSM bonding layers and core thickness plays an important role on the compressive strength. Analytical models are provided to predict the pure compressive and buckling strength, which are verified through FE results. Compression tests are further carried out to correlate with the analytical results. The number of the ChSM bonding layers and panel core thickness define each specimen type. Different failure modes are obtained for different parameter combinations, and their linear and failure responses are described.

The second objective is to study the core materials under out-of-plane shear. Analytical models are provided for shear buckling, pure shear failure, and debonding. Shear buckling can be solved using Rayleigh-Ritz method, and the latter two failure modes are based on accurate description of shear stiffness and stress distribution considering skin effect. Design formulas are provided to predict the failure strength. To verify the analytical models, a series of four-point bending tests are further carried out by varying the number of bonding layers and core thickness, both along longitudinal and transverse directions. 
Another objective is to study the strength properties of the facesheet. An FE progressive failure model is developed, which can be validated by the existing test results, and is further used to carry out a parametric study by varying material properties, layer thickness, and layer sequences. Compression and bending tests are carried out on selected layer configurations. An optimized facesheet configuration is recommended.

Finally, failure criteria are given for each type of failure mode and practical design formulas are provided for design purposes. Recommendations to improve the strength of HFRP panels are presented.

\subsection{Organization}

There are seven chapters in this dissertation. Chapter 1 includes problem statement, objective and scope of work, and the organization of the dissertation.

A literature review on sandwich panels under out-of-plane compression, out-of-plane shear, and FRP laminated plates is presented in Chapter 2.

In Chapter 3, analytical models to predict the compressive and buckling strength are provided, which are verified by FE results. An elastic restrain coefficient is introduced to quantify the bonding layer effect and simplified equations are formulated to predict the compressive strength. To further study the compressive behavior, two types of tests are carried out: namely stabilized and bare compression tests in order to achieve the pure compression and buckling failure. Close correlations among analytical, FE and test results can be obtained.

In Chapter 4, it is found that a complicated stress field at the interface resulted under shear, due to shear and bending warping effect induced by the facesheet. An analytical 
model is derived to calculate the stiffness as well as interfacial stresses, where the accuracy is proved against FE results. Rayleigh-Ritz method is employed to study shear buckling. Four-point bending tests were carried out to study the bonding layer and core thickness effect. Design formulas are provided to predict shear crushing, shear buckling and debonding, where the applicability is validated through the correlation with test results.

In Chapter 5, a progressive failure model using FE analysis is developed to predict the behavior of laminated composite plates up to failure, which is verified by the existing experimental data. A parametric study is carried out considering different laminate configurations. Three variables are included to define their effects: material properties; layer thickness; and layer sequences. Based on the FE results, bending and compression tests in accordance with ASTM standards were conducted on selected coupon samples. The FE model can also be validated by the test results. An optimized facesheet configuration is recommended.

In Chapter 6, based on the results of this study, failure criteria are proposed for critical failure modes. Also, design guidelines are provided for application in practice. An example is given illustrating the use of the design guidelines. Further, recommendations are provided for material architectures and core geometry configurations.

In the last chapter, major conclusions are summarized and suggestions for future investigations are also presented. 


\section{CHAPTER 2}

\section{LITERATURE REVIEW}

\subsection{Introduction}

As stated in Chapter 1, the objective of this study is the strength evaluation on core materials, facesheets, and the interface between core and facehsheet. Much effort has been devoted to the stiffness modeling and optimization of the HFRP sandwich panel shown in Figure 1.2. Davalos et al. (2001) developed equivalent orthotropic properties representative of the complex honeycomb geometry, and they presented a simplified analysis procedure that can be used in design applications. Xu et al. (2001) derived an analytical solution for the transverse shear stiffness of composite honeycomb with general configurations. However, no study is available on the strength properties of this HFRP sandwich structure, partly due to its complicated honeycomb core geometry. Therefore, there is a need to further characterize the strength of this product. Previous study by DeTeresa (1999) indicated that core materials for sandwich structures are primarily subjected to shear and through thickness compression. On that account, the focus of this chapter is to review mechanisms under such loading as well as to explain origins and rational for choosing to study the behavior of HFRP sandwich panels. In this vein, 
Section 2.2 reviews the previous work on core materials under out-of-plane compression,

Section 2.3 considers the work on core materials under out-of-plane shear, and Section 2.4 examines the significant issues pertaining to facehsheet study.

\subsection{Out-of-plane compression}

\subsubsection{Compressive Strength of Core Materials}

Chopped Strand Materials (ChSM) is used for the core materials which are composed of E-glass fiber and Polyester resin. In the aerospace industry, both modeling and testing of the strength of the composite materials have been achieved through its relatively long history of application. The modeling of ChSM can be dated back to the 1970's. Hann (1975) replaced the random composite by a laminate consisting unidirectional plies in every direction in the plan of laminate. Using maximum stress criterion, the strength of the random composite was given in terms of uni-axial strength of the unidirectional composite through a simple relation. Halpin and Kardos (1978) modeled the random fiber composites as a quasi-isotropic laminate consisting of $\left(0^{\circ} / 90^{\circ} / \pm 45^{\circ}\right)_{\mathrm{s}}$ plies. A maximum strain failure criterion was considered to predict the ultimate strength. They provided several examples illustrating the use of the model. Both of these studies treated the ChSM as layups of laminate in balanced condition, which are still in use (Barbero, 1999). 


\subsubsection{Core Crushing}

One of the common failure modes for sandwich structures under out-of-plane compression is core crushing. Theotokoglou (1996) offered an analytical determination of the ultimate strength of sandwich beams considering the core failure in compression, tension and shear using maximum failure strength method. He also performed a pull-out test to verify his model. However, his study only gave an indication of the failure modes that took place in a T-joint under pull-out load and further research was required in order to predict accurately the failure modes. Cvitkovich and Jackson (1998) studied the compression failure mechanisms in composite sandwich structures. The specimens in their study were tested with no damage, with 0.25 " diameter hole and with three levels of impact damage. Mouritz and Thomson (1999) investigated the compression, flexure and shear properties of a sandwich composite containing defects. They concluded that determining the compressive properties of a large sandwich structure was difficult because the strength and failure mechanism were dependent on the gauge length. Core crushing under compression was observed in all these studies.

\subsubsection{Buckling}

For HFRP sandwich panels used for bridge deck applications, the following distinct features characterize them from their counterparts in other fields; they have relatively larger and sparsely distributed honeycomb cells, and the core and facesheets are manufactured separately and subsequently connected by contact bonding, using a chopped strand mat $(\mathrm{ChSM})$ and polymer resin at the interface. In the literature, two 
types of FRP decks have been investigated and used in rehabilitation projects and new construction: (1) assemblies of bonded tabular or cellular sections (typically produced by pultrusion) of several shapes, such as truss (Brown et al., 1998), hexagonal (GangaRao et al., 1999), rectangular (Qiao et al., 2000), and tube (Kumar et al., 2003); and (2) sandwich panels using either foam or honeycomb cores. A recent review article describes commercially available FRP deck products of both types (Bakis et al., 2002). Therefore, due to the relatively low material stiffness and thin-walled sectional geometries of structural components, two possible instability problems for sandwich panels may result under different compression loading conditions. Specially, one is the wrinkling of the facesheet under in-plane compression (Niu and Talreja, 1998), and the other is the instability of the core due to out-of-plane compression (Zhang and Ashby, 1992). Out-ofplane compression is unavoidable in civil engineering applications, such as local compression on bridge decks exerted by wheel loads. The buckling of honeycomb core becomes more significant due to the sparsely distributed thin-wall core panels. As reported by Kumar et al. (2003), local buckling of the thin walls precipitated most failure modes in their bending tests of tube bridge decks. Thus, it is necessary to provide a solution for transverse buckling of core elements, for loaded edges partially restrained by the interface bond with the facesheet panels.

Zhang and Ashby (1992) concluded that two possible failure modes for out-ofplane compression were buckling and material crushing, or pure compression failure. In their study to predict buckling strength, they assumed the two edges of the core wall perpendicular to the loading direction as simply-supported, while the other two loaded edges as rigidly constrained. Their solution was later applied by Lee et al. (2002) to study 
the behavior of honeycomb composite core at elevated temperature. Both of these studies assumed a completely rigid connection at the facesheet-core interface, which is seldom the case in practice. The partial constraint offered by the interface bond has a significant effect on the behavior of FRP sandwich panels. This effect may vary due to different materials and manufacturing techniques used, with the clamped and hinged conditions as two extreme cases for the connection. In general, the quality of the bonding effect can be improved either by selecting compatible bonding materials or increasing embedment of the core into the bonding layer. The latter method is analogous to increasing the contact depth, or increasing bonding layer thickness, which in turn produces larger fillets of excess adhesive, which formed at honeycomb interfaces and effectively increasing the bonding area. This facesheet-core interaction is typically called the bonding layer effect. Burton and Noor (1997) used detailed FE models to examine the effect of the adhesive joint on the load transfer and static responses of sandwich panels. However, they used strain energy for discrete components to discuss the effect of various parameters, a method which cannot be readily used in practice. Up to now, the bonding effect on the behavior of honeycomb sandwich panels has not yet been clearly defined. It is the objective of this study to quantitatively study this effect on the behavior of sandwich panels under compressive load.

By considering the bonding layer effect, the problem can be interpreted as the instability of a partially restrained plate. The research on this topic can be traced back to the 1950's (Bleich, 1952). Recently Qiao et al. (2001) performed a study on the local buckling of composite FRP shapes by discrete plate analysis. They provided an explicit solution for the problem with elastic constraint along the unloaded edges, and also 
provided detailed references on this topic. Their research was further explored by Kollar (2002) and Qiao and Zou (2003). All of the previous studies are focused on the buckling of plates or panels with elastic restraint along the unloaded edges; this restraint is provided by connection of flange-to-web elements for beam-type members. However, for the HFRP core under out-of-plane compression, the two edges in contact with the facesheet panels (Fig. 1.2) can be treated as partially constrained; i.e., the elastic restraint is along the loaded edges. And this restraint results from the degree of connectivity between the facesheet and core. At present, there is no closed-form solution for this problem, and it is therefore advantageous to develop an analytical solution for compression buckling capacity of a plate with two loaded edges partially constrained.

In this study, analytical models are provided for the two failure modes: core crushing and buckling. The coefficient of elastic restraint is introduced to quantify the bonding layer effect. A comprehensive approach is developed to study the buckling behavior of HFPR core with varying degrees of boundary restraints, and an analytical solution is proposed by solving a transcendental equation. The bonding layer effect is evaluated experimentally by compression tests, which are designed such that buckling failure and pure compression failure can occur distinctly and separately. A novel testing method to predict the elastic constraint coefficient is also described, a parametric study is carried out to study the aspect ratio effect on the buckling behavior, and finally, design guidelines are proposed. 


\subsection{Out-of-plane Shear}

It is commonly believed that two failure modes may occur for a sandwich panel under out-of-plane shear: shear crushing (Allen, 1969; Vinson, 1999) and shear buckling (Qiao et al., 2001; Papadopoulos and Kassapoglou, 2004). Recently Chen and Davalos (2004) pointed out that the skin-effect can significantly affect interfacial stress distribution, yielding a coupled stress state, where the normal stress may even be larger than the shear stress. They concluded that, unlike the common belief that only shear stress occurs when the structure is under pure shear force, tensile force at the interface arises for a sandwich core, especially at the intersections of core elements, making such locations critical for debonding. Therefore, debonding may occur well before shear crushing or buckling is achieved.

To predict the shear strength of sandwich panel, accurately description of the stiffness is a prior. The computational models on honeycomb sandwiches are generally based on the equivalent replacement of each component with homogeneous continuum, due to expensive computation of 3-D detailed properties. Therefore, to accurately represent the equivalent properties has been a perennial challenging topic that attracted a lot of investigations. From Fig. 1.2, one can intuitively conclude that honeycomb sandwich structures behave like I-beams: the outer facesheets correspond to the flanges, and carry most of the direct compression/tension bending load, and the lightweight core corresponds to the I-beam web. The core supports the skins, increases bending and torsional stiffness, and carries most of the shear load (Noor et al., 1996). This characteristic of a three-layer arrangement leads to classical sandwich theory (Allen, 
1969; Zenkert, 1995). Unlike the facesheet, which can even be a laminated plate, the equivalent properties of honeycomb cores are more complicated.

\subsubsection{Stiffness Study on Equivalent Properties of Honeycomb Cores}

A lot of research has been devoted to this area. These include Warren and Kraynik (1987), Gibson and Ashby (1988), Fortes and Ashby (1999), and included in the book of Gibson and Ashby (1988) is the first systematic literature review in the field. All these mathematical models are based on pure cellular structures and the presence of the facesheet is not considered. As a result, the existing analytical solutions do not agree well with experimental results (Shi and Tong, 1995).

A comprehensive review of the computational models on honeycomb sandwiches was given by Noor et al. (1996), where numerous references were cited. Xu and Qiao (2002) provided a review specifically on stiffness studies of hexagonal honeycomb core. Basically, all existing studies can be organized into two groups:

Neglecting skin effect

The practice of neglecting skin effect is prevalent in today's sandwich research and design, wherein a uniform stress distribution in the walls of the structure is assumed. The in-plane elastic properties were first obtained by Gibson and Ashby (1988), where conditions of uni-axial loading and bi-axial loading were considered. Masters and Evans (1996) further refined the analysis attempting to consider stretching and hinging effects. Kelsey et al. (1958) firstly applied energy method to calculate the transverse shear stiffness, and showed that the theory of minimum potential energy, a kinematically 
compatible uniform strain field, gives an upper bound; and the theory of complementary energy, a statically compatible uniform stress field, gives a lower bound, corresponding to infinitely large (upper bound) and zero (lower bound) skin effect, respectively. The expressions for these two bounds were provided in terms of unit load and unit displacement method. Gibson and Ashby (1988) presented the predictions for transverse shear stiffness using mechanics of materials and energy method. In parallel to energy method, a good attempt was made by Shi and Tong (1995) in presenting an analytical solution for hexagonal honeycomb core using a 2-D homogenization method and obtaining the lower bound value. $\mathrm{Xu}$ et al. (2001) further extended it to general honeycomb configurations, where they developed an analytical approach with a twoscale asymptotic homogenization method.

\section{Considering skin effect}

As observed in experiments (Adams and Maheri, 1993; Daniel and Abot, 2000), skin constrain was demonstrated by the phenomenon of skin lateral contraction and expansion. Rather than assuming a uniform stress field, Penzien and Didriksson (1964) formulated a displacement field for transverse shear problem to simulate the warping effect induced by the facesheet. For the first time they showed the trend that as core height increases, the transverse shear stiffness decreases. Recently Xu and Qiao (2002) applied a multi-pass homogenization method to study the stiffness for transverse shear, in-plane stretch and out-of-plane bending. In both of these studies, the inclined panel was unfolded into the plane of flat panel, and therefore, the solution corresponds to a 2-D model. Grediac (1993) applied FE method to study core cells with different core 
configurations, and he studied the stress distribution in core walls. He concluded that the skin effect is a localized phenomenon limited only to the region adjacent to the interface. However, due to the cumbersome modeling work required by FE analysis, his study was only case-specific and not applicable to carry out general parametric studies.

In order to more accurately describe the elastic moduli of the core, Penzien and Didriksson (1964) introduced the concept of warping effect, or skin effect, into the model. Later Grediac (1993), Shi and Tong (1995), Becker (1998), and Xu and Qiao (2002) further considered this effect in their studies. It is interesting to point out that different researchers defined this effect in different ways, such as warping constraint by Penzien and Didriksson (1964), bending effect by Grediac (1993), thickness effect by Becker (1998), and skin effect by Xu and Qiao (2002). Recently Chen and Davalos (2004) decomposed this effect into shear and bending warping effects. However, all of these studies, either using 2-D model or FE method, were focused on the stiffness study only, and no work is available on the stress distribution at the interface, partly due to the following reasons: 1) the skin effect introduces a complicated stress field at the interface, which is difficult to model; and 2) unlike the modeling of stress distribution, acceptable results can be obtained for stiffness, which is a global property, even if an approximate displacement function is assumed. 


\subsubsection{Interfacial Stress Distribution}

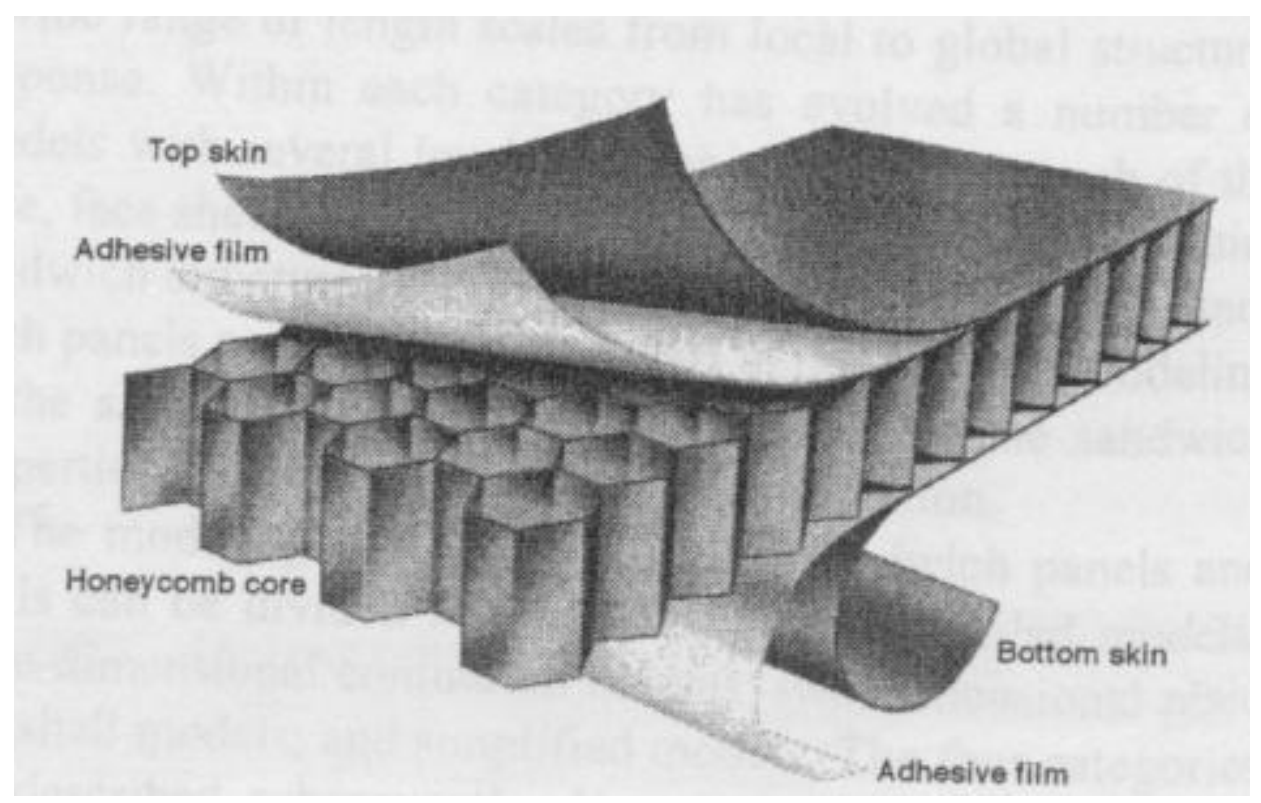

Figure 2.1 Sandwich panel with hexagonal honeycomb core (from Noor et al., 1996)

Several studies (e.g., Chen et al., 2003) have shown that delamination of the core from facesheet is a typical failure mode for sandwich panels. Fracture Mechanics method is usually adopted to study this problem, these include Ungsuwarungsri and Knauss (1987), Cui and Wisnom (1992), El-Sayed and Sridharan (2002), Blackman et al. (2003), Wang (2004) and other numerous works. It is shown by all these previous work that a crack is initiated when the interface traction attain the interfacial strength, and the crack is advanced when the work of traction equal to the material's resistance to crack propagation. Therefore, stress concentration at the interface can act as a criterion to predict the onset of the delamination, and there is a need to further investigate stress field at the interface. Chen and Davalos (2004) presented an analytical model allowing the 
calculation of the stiffness of honeycomb cores as well as the interfacial stress distribution considering skin effect, both under in-plane and out-of-plane forces, for hexagonal cores. To the best of the author's knowledge, accurate description of stiffness and interfacial stress distribution remains an open topic for HFRP sandwich panels with sinusoidal core. It is noted that the hexagonal cores (Fig. 2.1) are different from the sinusoidal cores (Fig 1.2) in that, for the hexagonal core, both the straight and inclined panels are affected by the skin effect. However, for sinusoidal core, due to the existence of the flat panel, only the sinusoidal panel is affected. The two modes, close cell and open cell configurations, are two major types that can be met for sandwich cores. Therefore, as a step further, the behavior of honeycomb sandwich panel with sinusoidal core geometry considering skin effect is presented in this study.

\subsubsection{Shear Crushing and Shear Buckling}

The concept of shear failure, including shear crushing or shear buckling is pretty straightforward. Allen and Feng (1998) defined three categories of sandwich panels: (1) composite beam theory (CBT), where the sandwich is treated as an ordinary composite beam and there is no shear deformation; (2) elementary sandwich theory (EST), where stresses and deflections are calculated by composite beam theory, but there is an additional shear deflection associated with shear strains in the core; and (3) advanced sandwich theory (AST), where the faces must bend locally in order to follow the shear deformation of the core. Most of the sandwich panels, including HFRP sandwich panels in this study, fall into the category of EST. One basic assumption used for EST is that the core resists the shear force and the facesheet carries the membrane forces caused by the 
bending moment (Allen, 1969; Vinson, 1999). It is shown (Caprino and Langella, 2000) that if the Young's modulus of the core is negligible with respect to the facing elastic modulus, and the facing thickness is small compared to the height of the core, the shear stress field in the core is practically uniform. Therefore, it is reasonable to assume that once this uniform shear stress exceeds the material shear strength, the panel will fail due to shear crushing.

The research on shear buckling problem has a relatively long history. Bleich (1952) firstly studied the shear buckling strength of metal structures. Timoshenko and Gere (1961) refined this theory and studied buckling of rectangular plates under action of shear stresses. Barbero and Raftoyiannis (1993) used the first variation of the total potential energy equation to study the shear buckling of FRP structures. Qiao et al. (2001) further applied this theory to study the local buckling of webs under shear loading. More recently, Papadopoulos and Kassapoglou (2004) developed a method based on a polynomial expansion of the out-of-plane displacement of the plate and energy minimization and studied the shear buckling of rectangular composites plates with two concentric layups. In all these studies, energy method is employed, and therefore, it is also adopted in this study. As pointed out earlier, two edges of the core panel are partially restrained. As a result, the potential energy will be given based on this boundary condition, and Rayleigh-Ritz method will be used to solve this problem.

\subsubsection{Testing Method}

To study the shear behavior of the sandwich core, ASTM (ASTM C273-00) specifies a testing method. However, this method cannot be directly applied to this study 
since the core is very strong in shear. Trial tests using this method illustrated that the failure is intra-laminar delamination, instead of pure shear failure of the core. Another method, four-point bending test is also recommended by ASTM (ASTM C393-00) to study shear strength and shear stiffness of HFRP sandwich cores since pure shear and bending regions will result from this loading condition, which is also adopted in this study. Many researchers have performed bending tests on sandwich beams. Lingaiah and Suryanarayana (1991) carried out experimental versus analytical correlation of the mechanical properties of sandwich-beam specimen. Four-point and three-point load tests were conducted. It was observed that generally the failure load was higher for the case of the four-point bending test than with three-point bending test. The failure of most of specimens was due to debonding between the core and the facing and at loads which were less than the theoretical estimated based on the allowable core shear stress or the allowable facing tensile/compressive stress, whichever was lower depending on the test condition. But they did not specify the position where the debonding initiated and did not look deeper into the mechanism behind the observed failure mode, where skin effect produces a tensile force in the pure shear region causing the facesheets debond from the core before the facesheet achieve their material strength. Mouritz and Thomson (1999) carried out four-point bending tests to study shear properties of a sandwich composite containing interfacial cracks subjected to impact load. They found that the composite containing the interfacial crack failed at a lower load than the defect-free specimen. The former failed due to a shear crack initiated near the interfacial crack tip, and upon loading grew into the foam until it reached the opposing skin, while skin wrinkling was a common failure mode in defect-free sandwich composite. The defect free sandwich 
composite did not fail by a shear or bending dominated process. The stiffness and strength of the sandwich composite decreased with increasing impact energy and impact damage area except when the composite was loaded in bending-tension. However, their tests were based on small coupon tests and were difficult to predict the properties of large structural components. Zenkert (1991) also observed the same type of shear failure in polymer foam sandwich composites containing interfacial cracks. Zenkert (1991), Triantofillou and Gibson (1989) and Thomson et al. (1989) have shown that the load needed to cause the onset of shear cracking can be predicted with good accuracy using analytical of FE models based on Mode II fracture mechanics theory applied to a layered anisotropic materials. Caprino and Langella (2000) performed three-point bending tests on a sandwich beam for the shear characterization of foam core. The special feature of their specimen was that they inserted rigid blocks in proximity to the concentrated load. They concluded that this method allowed for an accurate measurement of shear modulus and shear strength compared to ASTM standards. However, this method was very complicated and a lot of parameters should be calculated to design the test setup. Further test data need to be generated to assess the test for materials different from foam cores.

In this study, analytical models are presented to predict the strength due to pure shear crushing, shear buckling, and the delamination of the core from facesheet. The skin effect can be described through shear and bending warping effect. All previous studies on skin effect only considered the membrane force, which corresponds to shear warping defined herein. The bending warping effect is for the first time presented. The analytical 
models are verified through FE analysis. To further understand the behavior of core material under out-of-plane shear, four-point bending tests are carried out.

\subsection{Facesheet Study}

\subsubsection{Progressive Failure Analysis}

A lot of research has been done in the area of progressive failure analysis. The conventional strength analysis, called total-ply-discount (Vinson and Sierakowski, 1987), does not recognize that ply-failure is localized and therefore, it underestimates laminate strength. First-ply-failure (FPF) can be used to predict the onset of the damage (Barbero, 1999) as long as the stresses in each laminate are computed accurately. The objective of progressive failure analysis is focused on post-FPF analysis. According to Kim (1995), there are two approaches to include damage: modifying the stiffness matrix directly (Lee, 1982; Ochoa and Engblom, 1987; Hwang and Sun, 1989; Tolson and Zabaras, 1991) and degrading the material properties (Tan, 1991; Tan and Perez, 1993; Reddy and Reddy, 1993; and Kim, Davalos and Barbero, 1996).

Using stiffness modification approach, Lee (1982) developed a three dimensional FE computer program to analyze fiber-reinforced composite laminate. The program could calculate the detailed stress distribution, identify the damage zone and failure mode, analyze the damage accumulation, and determine the ultimate strength. He defined three types of damage: breakage of fibers, failure of matrix and delamination. The stresses at the center of each element were taken as the representative of that element for fiber breakage and matrix failure, and the stresses at the center of interface between two layers were taken as the representative stress for delamination. Based on the three damage 
types, the stiffness matrix was modified accordingly. He applied this program to study damage accumulation in composite laminate containing circular holes subjected to inplane loading. However, due to mesh coarseness at the edge of holes, delamination could not be captured. Further refinement of the finite element mesh was practically impossible due to computational limitations. Ochoa and Engblom (1987) used a higher-order plate element and computed transverse stresses from equilibrium equations. The failure analysis procedure was similar to that used by Lee (1982). Hwang and Sun (1989) developed an iterative 3-D finite element analysis with modified Newton-Raphson scheme for the failure prediction of laminates. Tolson and Zabaras (1991) followed a similar procedure to that used by Ochoa and Engblom (1987), using a higher-order plate element. Tsau and Plunkett (1993) investigated a square plate made of a layered composite material, with a centered circular hole subjected to in-plane biaxial loading, using a family of eight-node elements. Hashin failure criteria were adopted in his study and mesh size of FE model in laminates was carefully considered. In their analysis, at each increment of load, only one element, which was the one with the largest function value of the criterion in either fiber or matrix mode, was assigned to fail.

Using the material-degradation approach, Tan (1991) investigated the progressive failure with cut-out holes under in-plane tension testing. Different degradation factors were used for longitudinal modulus due to fiber breakage and transverse shear moduli due to matrix failure. The same approach was adopted by Tan and Perez (1993) to study the compressive loading case. Reddy and Reddy (1993) developed a three-dimensional progressive failure algorithm where the Layerwise Laminate Theory of Reddy was used for kinematic description. The stiffness of reduction was carried out at the reduced 
integration gauss points of the FE mesh depending on the mode of the failure. Two types of stiffness reduction methods were used: independent, i.e., each stress would contribute only towards degradation of the corresponding stiffness property; and interactive method, i.e., coupling was assumed between normal and shear stiffness properties. However, material properties were degraded by the same factor regardless of failure modes. They concluded that further investigation was required to apply their approach to laminates under compressive and bending load. Kim et al. (1996) formulated a Beam element with Layer-wise Constant Shear (BLCS) based on layer-wise laminated beam theory. Two schemes to predict load-displacement paths were used: load controlled and displacementcontrolled. The stiffness degradation factors were evaluated through parametric studies and correlation with experimental results. The BLCS predictions for ultimate loads and displacements were accurate compared to experimental results. However, when experimental responses showed non-linear load-displacement behavior, the prediction for displacement could not exactly match experimental results.

It is found out that most of the previous progressive failure analysis using FE is based on in-house programs, which requires a lot of efforts and time, and also the code developed by one researcher cannot be readily used by others. Nowadays, some general purpose FE analysis tools, such as ABAQUS, ANSYS, etc., are widely used in the academic and industrial field. These programs allow users to define their own subroutines in the analysis to fulfill the functions such as stiffness reduction and material degradation as described above. After evaluating all the possibilities, we choose to develop a progressive failure model through a user-defined subroutine using ABAQUS. 
One important issue in the progressive failure analysis is to find an appropriate failure criterion. Various failure criteria for isotropic or composite materials have been proposed. In general, the failure criteria are categorized into two groups: independent and polynomial failure criteria. A review of failure criteria of fibrous composite materials was given by Echaabi et al. (1996). The maximum stress and strain criteria belong to the first category, and they are simple to apply and can tell the mode of failure, but they neglect the stress interaction. An interactive criterion such as Tsai-Wu, Hoffman, or Hill, includes stress interaction in the failure mechanism, but it does not tell the mode of failure, and it requires some efforts to determine parameters such as $\mathrm{F}_{12}$ in Tsai-Wu criterion. Among others, Hashin (1980) provided a three dimensional failure criterion, which includes fiber tension, fiber compression, matrix tension, and matrix compression. This criterion not only considers the stress interaction, but provides the failure mode. Therefore, it is widely used (Spottswood and Palazotto, 2001; Kroll and Hufenbach, 1997) and is adopted in this study. However, Hashin (1980) did not specify the delamination criterion, which becomes significant when the laminate fails due to interlaminar shear failure. This issue was recently addressed by Elawadly (2003). Fortunately, Lee (1982) further proposed a delamination mode in his 3-D analysis, and is adopted in this study as an addition to Hashin's failure criterion.

Mostly commonly used FE model are 2-D (Kim, Davalos and Barbero, 1996) and 3-D analyses (Reddy and Reddy, 1993). For 2-D analysis, based on plane stress assumption, the transverse shear stresses, $\sigma_{13}$ and $\sigma_{23}$, and normal stress, $\sigma_{33}$, are neglected. As a result, the failure mode of delamination cannot be considered. 3-D progressive failure analysis was successfully developed by several researchers. However, 
the disadvantage is apparent. Take a 32-layer laminate as an example, the element will be 32 times of that in 2-D modeling, resulting in a cumbersome work both for modeling and computation, which hampers its use for a parametric study. Therefore, it is the objective of this study to develop a model that uses 2-D element and can still predict the delamination failure. Since $\sigma_{33}$ is negligible considering the thickness-to-length ratio for each layer, only $\sigma_{13}$ and $\sigma_{23}$ should be considered for delamination. In ABAQUS (2002), transverse shear stresses are not readily available in the output stress components for a shell element. Instead, they are stored in the result file as TSHR13 and TSHR23. Therefore, a user-defined subroutine is firstly employed to retrieve the transverse shear stresses from the result file. Combining with another subroutine to implement the failure criterion, the progressive failure analysis can be carried out.

\subsubsection{Testing Method}

A lot of tests have been carried out in this area. Standardized test methods (ASTM designations) were adopted in most studies. Cui et al. (1992) compared three- and fourpoint bending tests both analytically and experimentally. They concluded that, in all the three-point bending tests, damage was observed under the loading roller in addition to the inter-laminar shear failure, while in the four-point bending tests, only inter-laminar shear failure was observed. Kim and Crasto (1992) carried out a series of tests on a novel miniature sandwich specimen developed to measure composite compressive strength. The mini-sandwich beam specimens consisted of thin composite skins on both sides of a core made of materials similar to matrix resin. The advantage of this method was that it can avoid the premature buckling failure. They concluded that the compressive strength 
determined in this study was approximately equal to the tensile strength. But the sandwich panel fabrication was a two-step process and required more time and effort than a conventional testing methods. Grief and Chapon (1993) conducted three-point bending tests on laminated composite beams and attempted to predict successive failures. Five composites laminate types were used with different lay-ups. Fiber breakage, matrix damage, and delamination were observed during the test. They tried to used total-plydiscount failure analysis, that is, after a ply-failure, the analysis was repeated for a new laminate, in which the stiffness of a failed ply was set to zero, to predict the failures. However, their analytical predictions did not match the experimental results. LopezAnido et al. (1995) performed three-point bending tests, both flatwise (out-of-plane) and edgewise (in-plane), on rectangular lay-up angle ply $\left( \pm 45^{\circ}\right) \mathrm{s}$ beam elements. They concluded that the analysis based on the computation of the apparent lamina moduli provided a lower bound and that based on plane strain assumptions represented an upper bound for the beam stiffness. The threshold aspect-ratio that limits the range of application of various analytical methods was provided. Barbero et al. (1999) developed a fixture for testing compressive strength of coupon samples and pultruded structural shapes. Using this fixture, splitting at the end of the sample was prevented while reducing stress concentration at the ends, yielding compression failures at the center of specimen. All the fiber reinforcement of structural shapes (CSM, $45^{\circ}$, and roving) were tested individually and combined to support the development of a simple model for compressive strength of structural shapes. Waas and Schultheisz (1996) gave a good review on the experimental studies on compressive failure of composites. The factors affecting the compressive strength, such as matrix effects, interface effects, void content, etc., were 
discussed in detail through experimental results. They also correlated compressive strength with other properties and recommended testing techniques that may provide further insight into the mechanism that control composite compressive failures including simple microscopic observation, more sensitive interferometric methods, and to monitor acoustic emission. Unlike bending and compression, tension tests are less reported due to its easy implementation.

For HFRP sandwich panels, the face laminate may subject to tensile, compressive or bending forces depending on the loading conditions, where compressive force is more critical. Therefore, we need to evaluate the strength properties of face laminate through a combination of compressive and bending tests. The test results can also be used to verify the accuracy of the progressive failure model developed. 


\section{CHAPTER 3}

\section{OUT-OF-PLANE COMPRESSION}

\subsection{Introduction}

A combined analytical and experimental study of FRP sandwich panel under out-ofplane compression is presented in this chapter. Two analytical models, corresponding to pure compression and elastic buckling failure, respectively, are provided first. The sandwich panel consists of top and bottom laminated facesheets bonded to the honeycomb core, which extends vertically between facesheets. The facesheet and core are attached by contact molding and are, therefore, not rigidly connected. Thus, the buckling problem can be described as the instability of an FRP core panel with two rotationally restrained loaded edges. An elastic restraint coefficient is introduced to quantify the bonding layer effect between the facesheet and core, and a simple and relatively accurate test method is proposed to obtain the restraint coefficient experimentally. By solving a transcendental equation, the critical compression buckling stresses are obtained, and a simplified expression to predict buckling strength is formulated in terms of the elastic restraint coefficient. The analytical solution is verified by FE analysis. Compression tests were carried out to evaluate the effect of the bonding layer thickness and core thickness, and the experimental results correlate closely with analytical and FE predictions. A parametric 
study is conducted to study the core aspect ratio effect on the buckling load. Finally design equations are provided to calculate the compressive strength.

\subsection{Analytical Models}

From literature review, we conclude that there are two failure modes for HFRP sandwich panels under out-of-plane compression, i.e., pure compression and buckling failure. Correspondingly, two models are provided.

\subsubsection{Pure Compression Failure}

For this case, the nominal failure load can be calculated as

$$
F_{c}=f_{c} \times A_{c}
$$

where $f_{c}$ is the material compressive strength of ChSM, and $A_{c}$ is the total in-plane area of the core walls. 


\subsubsection{Buckling of Plate with Partially Constrained Loaded Edges}

\subsubsection{Analytical Model}

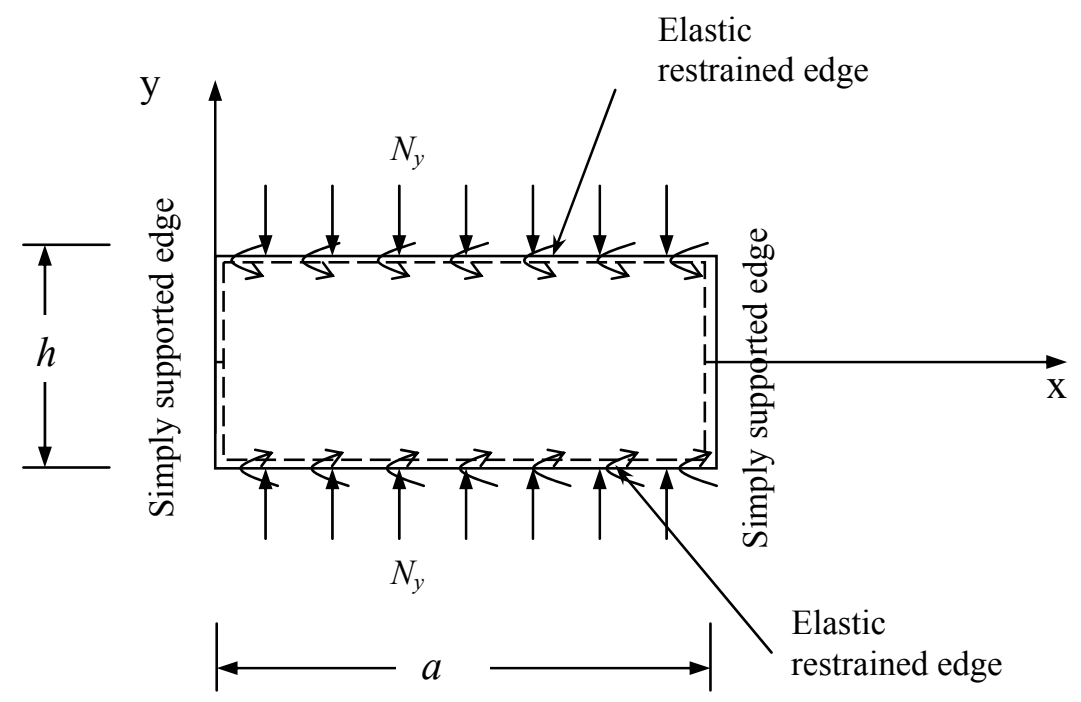

Figure 3.1 Boundary condition of FRP plate

The local buckling of core panels under uniformly distributed compression loading is analyzed in this section. Clearly, the core flat panels are more sensitive to buckling than the sinusoidal panels (Fig 1.2). Therefore, the problem can be simplified as the buckling response of the flat panel under in-plane compression. As the flat panel extends along the length of the core, it is reasonable to assume the connection edge between the flat panel and sinusoidal panel to be simply supported, as the natural location of a contra-flexure point. The boundary conditions are shown in Fig. 3.1. Two edges parallel to the loading direction are simply supported and the other two loaded edges are partially constrained. 
The governing differential equation for buckling of a symmetric anisotropic plate under in-plane axial loading is expressed as

$$
\begin{aligned}
& D_{11} \frac{\partial^{4} w}{\partial x^{4}}+4 D_{16} \frac{\partial^{4} w}{\partial x^{3} \partial y}+2 D_{12} \frac{\partial^{4} w}{\partial x^{2} \partial y^{2}}+4 D_{66} \frac{\partial^{4} w}{\partial x^{2} \partial y^{2}} \\
& +4 D_{26} \frac{\partial^{4} w}{\partial x \partial y^{3}}+D_{22} \frac{\partial^{4} w}{\partial y^{4}}+N_{y} \frac{\partial^{2} w}{\partial y^{2}}=0
\end{aligned}
$$

where $D_{i j}(i, j=1,2,6)$ are plate bending stiffness coefficients; $N_{y}$ is the in-plane uniformly distributed compressive stress resultant; and $w(x, y)$ is the buckled shape function of the plate. If the balanced symmetric condition is considered and no bendingtwisting coupling exists, then Eq. (3.2) can be simplified as

$$
D_{11} \frac{\partial^{4} w}{\partial x^{4}}+2 D_{12} \frac{\partial^{4} w}{\partial x^{2} \partial y^{2}}+4 D_{66} \frac{\partial^{4} w}{\partial x^{2} \partial y^{2}}+D_{22} \frac{\partial^{4} w}{\partial y^{4}}+N_{y} \frac{\partial^{2} w}{\partial y^{2}}=0
$$

Considering the boundary condition in Fig. 3.1, we can assume the shape function to be

$$
w=y \sin \frac{n \pi x}{a}
$$

Then Eq. (3.3) can be further simplified to

$$
D_{22} y^{(4)}+\left[N_{y}-\left(2 D_{12}+4 D_{66}\right)\left(\frac{n \pi}{a}\right)^{2}\right] y^{\prime \prime}+\left(\frac{n \pi}{a}\right)^{4} D_{11} y=0
$$


Introducing the following coefficients as

$$
\alpha=\frac{D_{12}+2 D_{66}}{D_{22}} \quad \beta=\frac{D_{11}}{D_{22}} \quad 2 \mu^{2}=\left(\frac{a}{n \pi}\right)^{2} \frac{N_{y}}{D_{22}}
$$

Eq. (3.5) becomes

$$
y^{(4)}+2\left(\mu^{2}-\alpha\right)\left(\frac{n \pi}{a}\right)^{2} y^{\prime \prime}+\left(\frac{n \pi}{a}\right)^{4} \beta y=0
$$

Apparently Eq. (3.7) is a typical fourth-order differential problem. The characteristic equation of this problem is

$$
r^{4}+2\left(\mu^{2}-\alpha\right)\left(\frac{n \pi}{a}\right)^{2} r^{2}+\left(\frac{n \pi}{a}\right)^{4} \beta=0
$$

The final form of the solution to Eq. (3.7) depends on the value of $\left(\mu^{2}-\alpha\right)^{2}-\beta$.

We can assume

$$
\left(\mu^{2}-\alpha\right)^{2}-\beta=0
$$

Substituting Eq. (3.6) into Eq. (3.9), we can get 


$$
\left[\left(\frac{a}{n \pi}\right)^{2} \frac{N_{y}}{2 D_{22}}-\frac{D_{12}+2 D_{66}}{D_{22}}\right]^{2}-\frac{D_{11}}{D_{22}}=0
$$

Solving for Eq. (3.10), we have

$$
N_{y}=\frac{2 n^{2} \pi^{2}}{a^{2}}\left[\sqrt{D_{11} D_{22}}+\left(D_{12}+2 D_{66}\right)\right]
$$

This is a well-known expression for the critical local buckling strength of a simply supported plate with $n$ half-waves in the $x$ direction (Reddy, 1999). For the problem considered in this study, for a given $n$, we always have $N_{c r} \geq N_{y}$, and therefore, we always have

$$
\left(\mu^{2}-\alpha\right)^{2}-\beta \geq 0
$$

Then the four roots of Eq. (3.8) are the complex numbers

$$
r= \pm \frac{k_{1} n \pi}{a} i ; \quad \pm \frac{k_{2} n \pi}{a} i
$$

where $\mathrm{k}_{1}, \mathrm{k}_{2}$ and $\mathrm{k}_{3}$ are defined as 


$$
\begin{aligned}
& k_{1}=\sqrt{\mu^{2}-\alpha+k_{3}} \\
& k_{2}=\sqrt{\mu^{2}-\alpha-k_{3}} \\
& k_{3}=\sqrt{\left(\mu^{2}-\alpha\right)^{2}-\beta}
\end{aligned}
$$

Then the solution for Eq. (3.7) takes the form

$$
w(x, y)=\sin \frac{n \pi x}{a}\left(C_{1} \cos \frac{k_{1} n \pi y}{a}+C_{2} \sin \frac{k_{1} n \pi y}{a}+C_{3} \cos \frac{k_{2} n \pi y}{a}+C_{4} \sin \frac{k_{2} n \pi y}{a}\right)
$$

As indicated in Fig. 3.1, the origin of the coordinates $\mathrm{x}$ and $\mathrm{y}$ is located at the mid-point of the left edge. Assuming equal elastic constraint on both loaded edges, the deflection function of $w$ is a symmetric function of $y$ when the plate reaches the critical buckling load. Therefore, Eq. (3.15) reduces to

$$
w(x, y)=\sin \frac{n \pi x}{a}\left(C_{1} \cos \frac{k_{1} n \pi y}{a}+C_{3} \cos \frac{k_{2} n \pi y}{a}\right)
$$

The boundary conditions can be described as

$$
w_{y= \pm h / 2}=0
$$

The rotational angle is assumed to be proportional to the edge moment, 


$$
M_{y= \pm h / 2}=-\bar{\zeta} \bar{\varphi}
$$

where $\bar{\varphi}$ is the rotation of the plate along the edges $y=h / 2$.

Based on the constitution equation of a laminated panel, and considering $\left(\partial^{2} w / \partial x^{2}\right)_{y= \pm h / 2}=0$, the moment $M_{y}$ is expressed as

$$
M_{y= \pm h / 2}=-D_{22}\left(\frac{\partial^{2} w}{\partial y^{2}}\right)_{y= \pm h / 2}
$$

Combining Eq. (3.18) and Eq. (3.19), we have

$$
\bar{\varphi}=\frac{D_{22}}{\bar{\zeta}} \frac{\partial^{2} w}{\partial y^{2}}
$$

A nondimensional factor or coefficient of elastic restraint (CER) is defined as

$$
\zeta=-\frac{D_{22}}{\bar{\zeta}} \frac{2}{h}
$$

Considering $\bar{\varphi}=\partial w / \partial y$, the boundary condition along the edges $y= \pm h / 2$ becomes

$$
\frac{\partial w}{\partial y}=\frac{h}{2} \zeta \frac{\partial^{2} w}{\partial y^{2}}
$$


The buckled shape function of Eq. (3.16) in combination with Eq. (3.17) and Eq. (3.22) results in homogeneous equations in terms of two constants $C_{1}$ and $C_{3}$. When the determinant of the coefficient matrix equals zero, the buckling criterion for a plate under equal elastic constraint on both loaded edges is established as

$$
\left|\begin{array}{cc}
\cos \frac{k_{1} n \pi h}{2 a} & \cos \frac{k_{2} n \pi h}{2 a} \\
-\frac{k_{1} n \pi}{a} \sin \frac{k_{1} n \pi h}{2 a}-\zeta \frac{h}{2}\left(\frac{k_{1} n \pi}{a}\right)^{2} \cos \frac{k_{1} n \pi h}{2 a} & -\frac{k_{2} n \pi}{a} \sin \frac{k_{2} n \pi h}{2 a}-\zeta \frac{h}{2}\left(\frac{k_{2} n \pi}{a}\right)^{2} \cos \frac{k_{2} n \pi h}{2 a}
\end{array}\right|=0
$$

Furthermore, Eq. (3.23) is simplified to a transcendental equation as

$$
k_{1} \sin \frac{k_{1} n \pi h}{2 a} \cos \frac{k_{2} n \pi h}{2 a}-k_{2} \sin \frac{k_{2} n \pi h}{2 a} \cos \frac{k_{1} n \pi h}{2 a}+\zeta \frac{n \pi h}{a} k_{3} \cos \frac{k_{1} n \pi h}{2 a} \cos \frac{k_{2} n \pi h}{2 a}=0
$$

A Fortran program is compiled to solve this equation, as shown in Appendix A. As pointed out by Reddy (1999), for a simply supported plate under uni-axial compression, the buckling load is a minimum when the half wave along unloaded direction is 1 . The theory also applies to this model. It is found out that $n=1$ always gives the minimum buckling load, while the number of half waves along the other direction can be calculated by the program for corresponding buckling load. 


\subsubsection{Verification with FE Simulation}

To verify the model derived in Section 3.2.2.1, both Eq. (3.24) and the FE method are used to predict the local buckling strength of the core panel under out-of-plane compression. The structure is a typical single cell of the honeycomb sandwich structure. This cell is 4" by 4" square and 2" deep, and the core thickness is $t=0.09$ ", as shown in Fig. 3.2. Table 3.1 lists stiffness properties of the core wall.

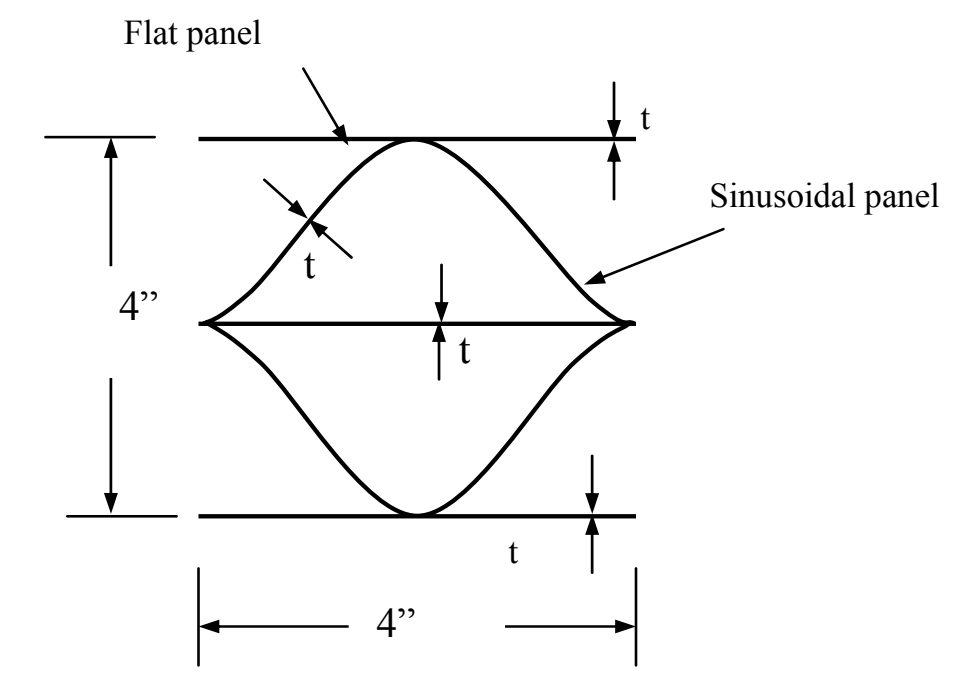

Figure 3.2 In-plane core specimen dimensions

Table 3.1 Properties of the core material

\begin{tabular}{|c|c|c|c|c|c|c|c|}
\hline $\begin{array}{c}\text { Ply } \\
\text { name }\end{array}$ & Orientation & $\begin{array}{c}\mathrm{E}_{1} \\
\left(\mathrm{x} 10^{6} \mathrm{psi}\right)\end{array}$ & $\begin{array}{c}\mathrm{E}_{2} \\
\left(\mathrm{x} 10^{6} \mathrm{psi}\right)\end{array}$ & $\begin{array}{c}\mathrm{G}_{12} \\
\left(\mathrm{x} 10^{6} \mathrm{psi}\right)\end{array}$ & $\begin{array}{c}\mathrm{G}_{23} \\
\left(\mathrm{x} 10^{6} \mathrm{psi}\right)\end{array}$ & $v_{12}$ & $v_{23}$ \\
\hline Core & Random & 1.71 & 1.71 & 0.61 & 0.43 & 0.402 & 0.388 \\
\hline
\end{tabular}

ABAQUS (2002) is adopted for FE analysis, and FEMAP (2001) is used for the pre- and post-processing. The modeling of the complex shape of sinusoidal wave is 
accomplished by exporting the geometry from AUTO-CAD. The core walls are modeled with a four-node shell element, S4. The global element size is chosen as 0.2 ". It has been checked through a convergence study that the present mesh provides an accurate value.

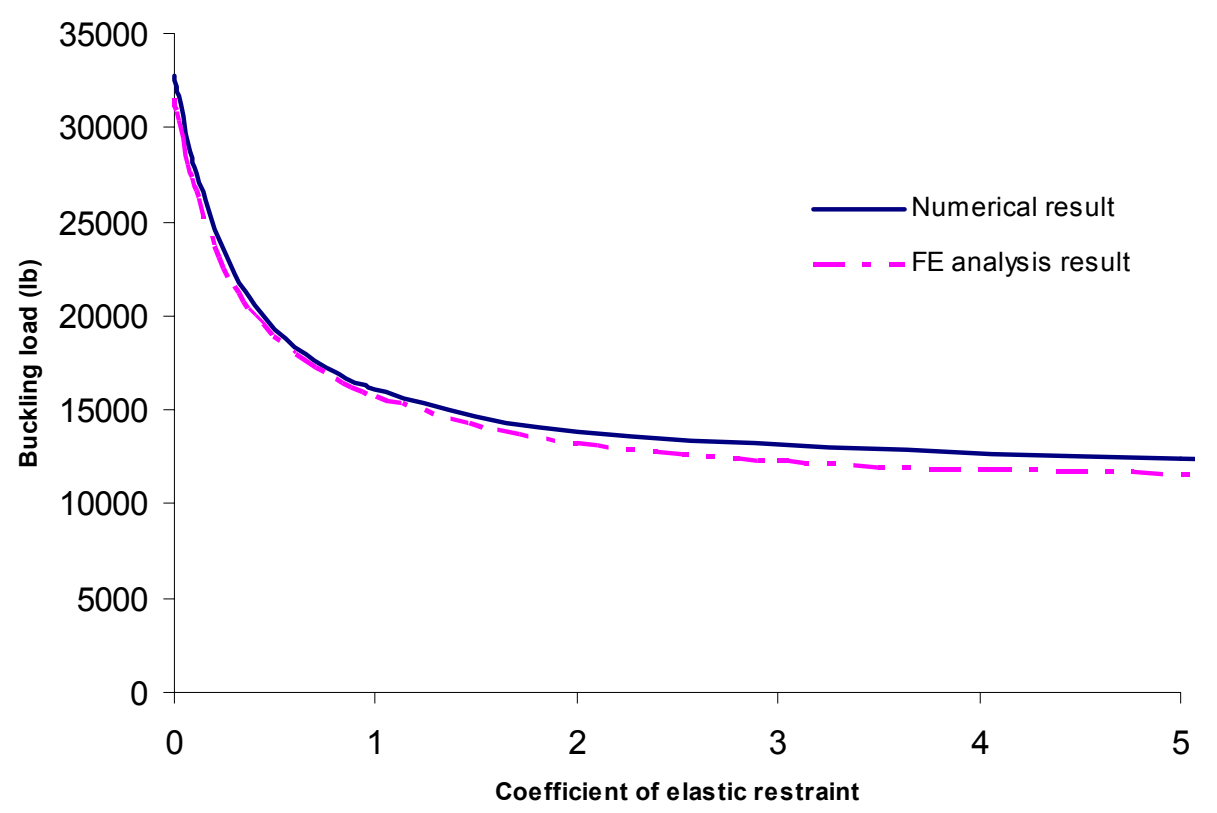

Figure 3.3 Buckling load vs. elastic restraint coefficient

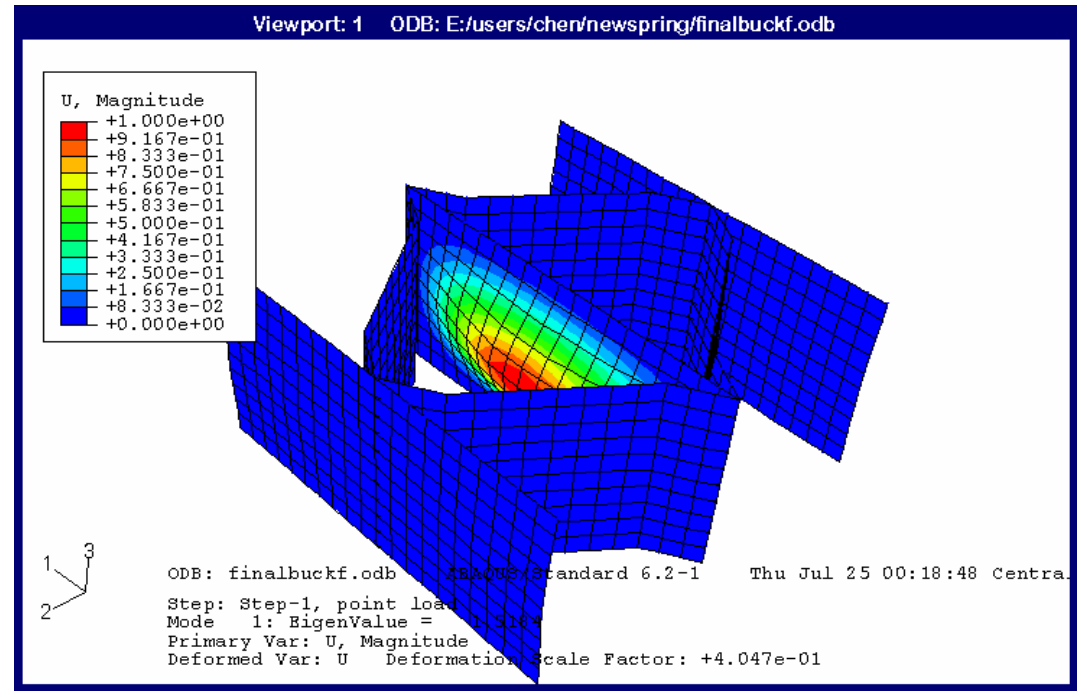

Figure 3.4 The first buckling mode for clamped condition 
In the FE analysis, the CER introduced in section 3.2.2.1 is adopted to account for the bonding layer effect, and a spring model is used to simulate the elastic constraint. Each node between the facesheet and the core is duplicated, and six spring elements, representing the constraints in six directions are placed in between. The normal spring stiffness is set to be a very large value. This dummy value prevents the core from detaching from the facesheet. The rotational stiffness is varied to represent the relative constraining condition, corresponding to a particular elastic restraint coefficient. An eigenvalue analysis is carried out, where the load corresponding to the first buckling mode is considered as the buckling load. It is shown that the buckling load is dependent on CER, and by varying CER $\zeta$, we can plot the buckling load as shown in Fig. 3.3, with the first buckling mode from the FE analysis illustrated in Fig. 3.4.

Solving for Eq. (3.24), we can obtain the buckling load $N_{y}$ for the flat panel in the cell, which is 4 " wide and 2" deep. If the compressive stress is assumed to be evenly distributed for the whole structure, multiplying $N_{y}$ by the total length of all the core walls, we can plot the buckling load versus elastic restraint coefficient in Fig. 3.3, from which it is shown that the analytical model fits the FE result quite well. When the coefficient of elastic restraint is assumed to be very large, which approaches a hinged connection, Eq. (3.24) gives the result of $\mu^{2}=3.125$, and substituting this value into Eq. (3.6), $N_{y}$ can be calculated as

$$
N_{y}=2 \mu^{2} \frac{\pi^{2} D_{22}}{a^{2}}=\frac{6.25 \pi^{2} D_{22}}{a^{2}}
$$


which corresponds to the solution given by Reddy (1999) for a plate under in-plane compression with four sides simply supported, leading to the solution

$$
N_{c r}=\frac{\pi^{2} D_{22}}{a^{2}}\left(\frac{a}{h}+\frac{h}{a}\right)^{2}=\frac{6.25 \pi^{2} D_{22}}{a^{2}}
$$

which is identical to Eq. (3.25), thus indirectly verifying the accuracy of the above formulation.

Table 3.2 Comparison between FE and analytical result

\begin{tabular}{|c|c|c|c|c|}
\hline & $F_{c r}^{\infty}(\mathrm{lb})$ & $F_{c r}^{0}(\mathrm{lb})$ & $\mathrm{p}$ & $\mathrm{q}$ \\
\hline FE result & 10,198 & 31,550 & 2.94 & 1.01 \\
\hline Analytical result & 11,249 & 32,737 & 3.48 & 1.07 \\
\hline
\end{tabular}

Table 3.3 Comparison between FE and analytical result for multi-cell panel

\begin{tabular}{|c|c|c|c|}
\hline & & $F_{c r}^{\infty}(\mathrm{lb})$ & $F_{c r}^{0}(\mathrm{lb})$ \\
\hline \multirow{2}{*}{$\begin{array}{c}2 \times 2 \text { cells } \\
(8 " x 8 ")\end{array}$} & FE result & 42,020 & 121,762 \\
\cline { 2 - 4 } & Analytical result & 41,204 & 119,902 \\
\hline $3 \times 3$ cells & FE result & 89,431 & 245,917 \\
\cline { 2 - 4 }$(12 " x 12 ")$ & Analytical result & 89,741 & 261,146 \\
\hline
\end{tabular}

For a given CER $\zeta$, we can get the buckling load correspondingly from the curves shown in Fig. 3.3. To simplify this procedure, we provide an explicit expression to predict the buckling load, which can also act as a design equation. Previous investigations (Qiao 
et al., 2001) showed that the buckling load vs. $\zeta$ curve shown in Fig. 3.3 can be fitted using the following equation

$$
\frac{F_{c r}-F_{c r}^{\infty}}{F_{c r}^{0}-F_{c r}^{\infty}}=\frac{1}{p \zeta^{q}+1}
$$

where $F_{c r}^{\infty}$ and $F_{c r}^{0}$ are critical loads corresponding to the hinged $(\zeta=\infty)$ and clamped $(\zeta=0)$ boundary conditions, respectively. They can be obtained from the analytical solution and FE analysis and are listed in Table 3.2. The parameters $p$ and $q$ can be determined from Eq. (3.24) by a regression technique, and the results from both the FE and analytical solutions are given in Table 3.2.

To further verify the analytical solution, panels composed of $2 \times 2=4$ ( 8 " $\times 8$ ") and $3 \times 3=9$ (12"x12") cells with the same core height are analyzed under compressive load. For simplicity, only the two extreme cases of hinged and clamped conditions are illustrated. The results given in Table 3.3 show that the analytical solution correlates well with FE results. 


\subsection{Experimental Investigation}

To further study the behavior of sandwich panels under out-of-plane compression, experimental investigation was carried out; two types of test, stabilized and bare compression test were conducted in order to achieve the pure compression and buckling failure as described in Section 3.2.

\subsubsection{Naming Conventions}

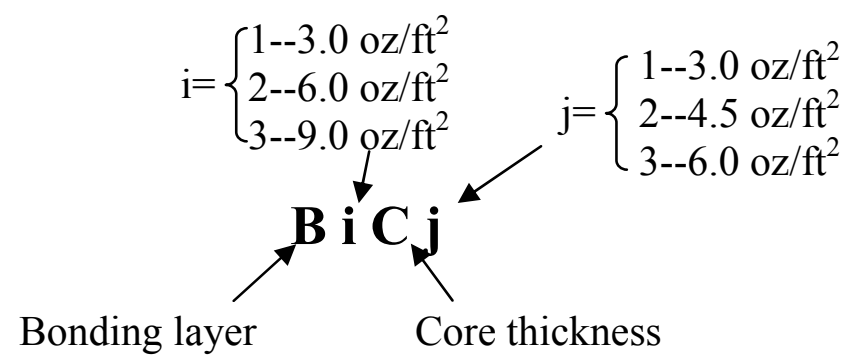

\section{Figure 3.5 Naming conventions}

Throughout this study, the naming conventions are defined in Fig. 3.5, where B and $\mathrm{C}$ represent Chopped Strand Mat (ChSM) Bonding layer numbers and Core thickness, respectively, and different value for $\mathrm{i}$ and $\mathrm{j}$ corresponds to different nominal weight of the ChSM. 


\subsubsection{Test Description}

The specimen is a typical single cell cut from the sandwich structure, which represents the weakest part of the structure when under compression. It is 4" by 4 " square and 2" deep, as shown in Fig. 3.2. To assess the effect of bonding layers and minimize the influence of the other layers of the facesheet, only three layers are selected for the facesheet, as shown in Fig. 3.6. The thickness of bonding layers is varied from one bonding layer to three bonding layers, and the core thickness is varied from one to two core thickness for different type of specimens. The constituent materials of the facesheet are given in Fig. 3.6. The properties of the constituent materials are provided in Table 3.4, and Table 3.5 lists the properties of each component material.

Table 3.4 Properties of constituent materials

\begin{tabular}{|c|c|c|c|c|}
\hline Material & $\mathrm{E}\left(\mathrm{x} 10^{6} \mathrm{psi}\right)$ & $\mathrm{G}\left(\mathrm{x} 10^{6} \mathrm{psi}\right)$ & $v$ & $\rho\left(\mathrm{lb} / \mathrm{in}^{3}\right)$ \\
\hline E-glass fiber & 10.5 & 4.18 & 0.255 & 0.092 \\
\hline Polyester resin & 0.734 & 0.237 & 0.3 & 0.041 \\
\hline
\end{tabular}

Table 3.5 Layer properties of face laminate and core materials

\begin{tabular}{|c|c|c|c|c|}
\hline Ply name & Ply type & Nominal weight $\left(\mathrm{oz} / \mathrm{ft}^{2}\right)$ & Thickness (in.) & $\mathrm{V}_{\mathrm{f}}$ \\
\hline Bonding layer & ChopSM & 3.0 & 0.082 & 0.1726 \\
\hline UM1810 & $0^{\circ}$ & 2.0 & 0.025 & 0.3774 \\
\hline & ContSM & 1.0 & 0.0132 & 0.3582 \\
\hline Core & ChopSM & 4.5 & 0.09 & 0.2289 \\
\hline
\end{tabular}




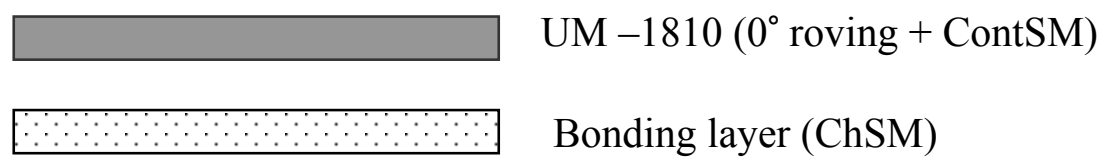

Figure 3.6 Lay-up of facesheet

Two cases of compression tests were carried out. For the first case of test, an elastic pad was placed between the loading block and the specimen; this method is known as bare compression test. For the second case of test, the specimen was bonded to top and bottom steel plates, and the load was applied directly over the steel plate; this method is called stabilized compression test. The bare compression test is more representative of actual patch loading conditions. The stabilized compression test is intended to minimize buckling effect and induce primarily compression failure.

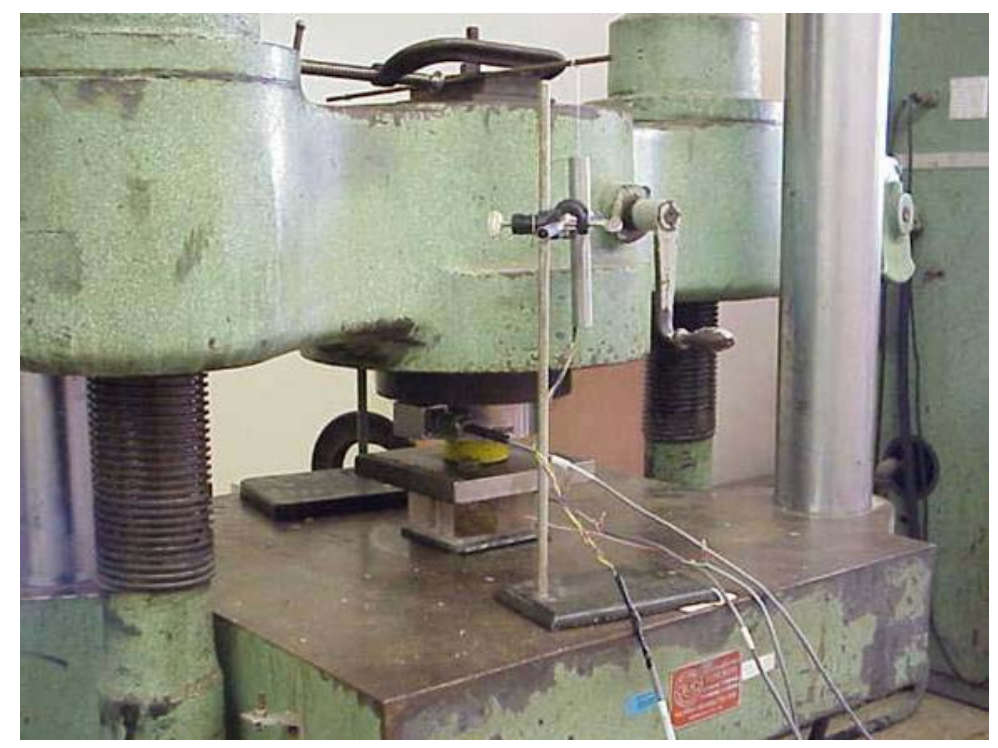

Figure 3.7 Compression test setup 
All tests were carried out according to ASTM standards (see Fig. 3.7). They were performed in a universal testing machine with a 200,000 lb capacity. A load cell was placed between the loading block and the specimen to record the load, and LVDTs were used to record the displacements. Four strain gages were bonded at the mid-height of the core to obtain compressive strains, two on the sinusoidal wave panel and two on the side flat panel (Fig. 3.2). The load was controlled at such a rate that the failure occurred within 3 to 6 minutes.

\subsubsection{Test Results and Discussion}

\subsubsection{Bare Compression Test}

When the load is applied to the specimen, both side flat panels bend outwards, and this deformation can be interpreted as a geometric imperfection. As the load increases, the specimens with distinct bonding layers display different behaviors. For $\mathrm{B} 1 \mathrm{C} 2$, the side panels buckle and delaminate from the specimen well before ultimate failure occurs. While for other types, the side panels do not delaminate. For all specimen types, upon sudden crushing of the side panel, the specimen does not fail immediately but continues to carry load for several event failures, until collapse of the specimen. A typical failure mode is shown in Fig. 3.8. 


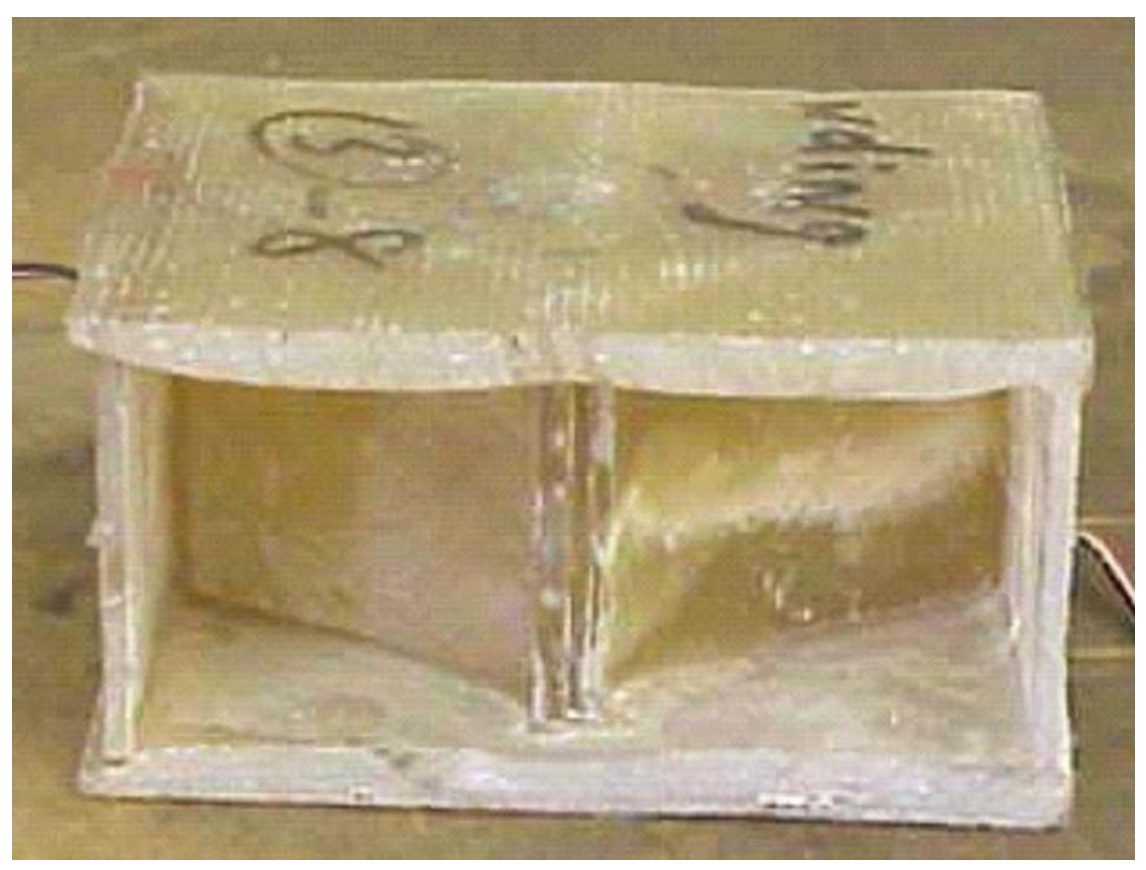

Figure 3.8 Bare compression test specimen

Table 3.6 Average value and standard deviation of failure load for bare compression tests

\begin{tabular}{|c|c|c|c|c|}
\hline & B1C2 & B2C2 & B3C2 & B3C1 \\
\hline Average value (lb) & 16,770 & 21,010 & 22,900 & 7,135 \\
\hline Standard deviation (lb) & 875 & 1,905 & 2,120 & 775 \\
\hline
\end{tabular}




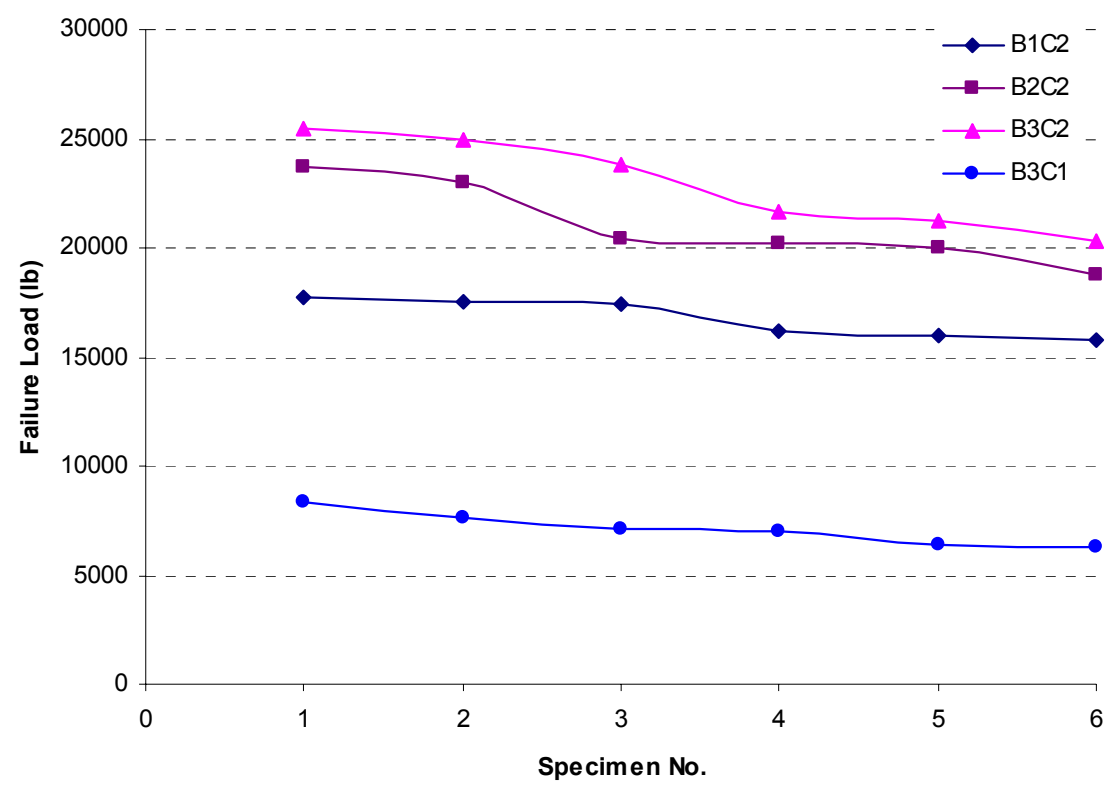

Figure 3.9 Failure load for bare compression test

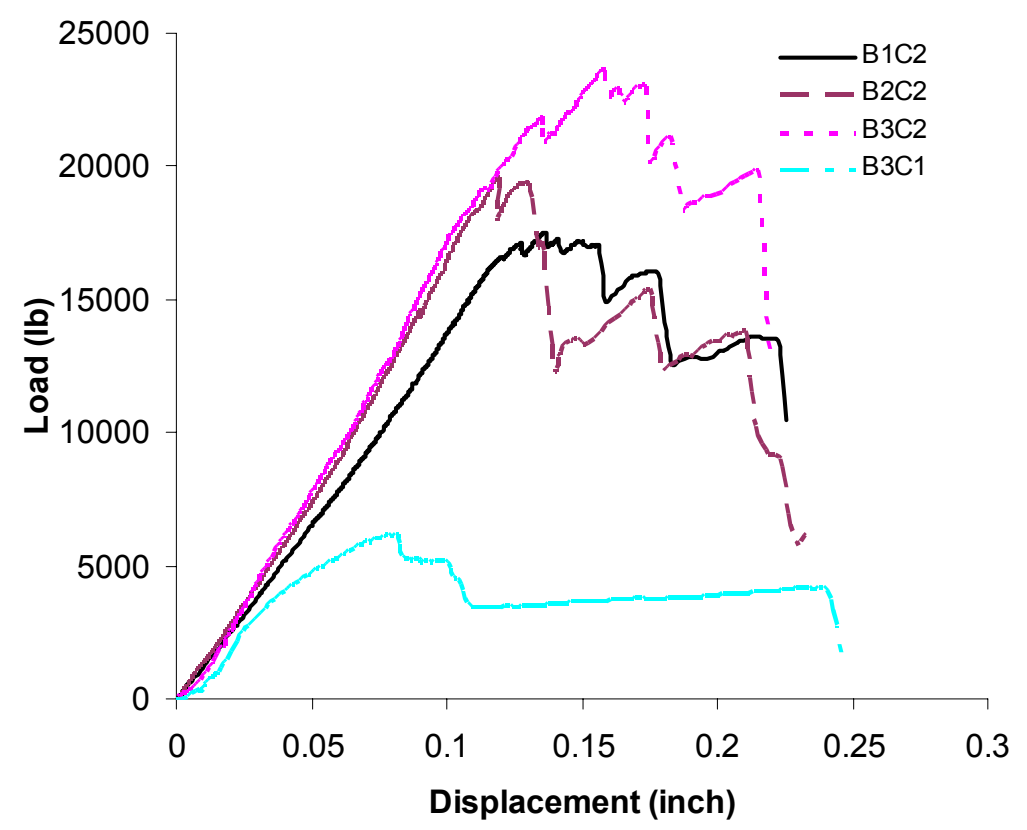

Figure 3.10 Load-displacement curve for bare compression test 


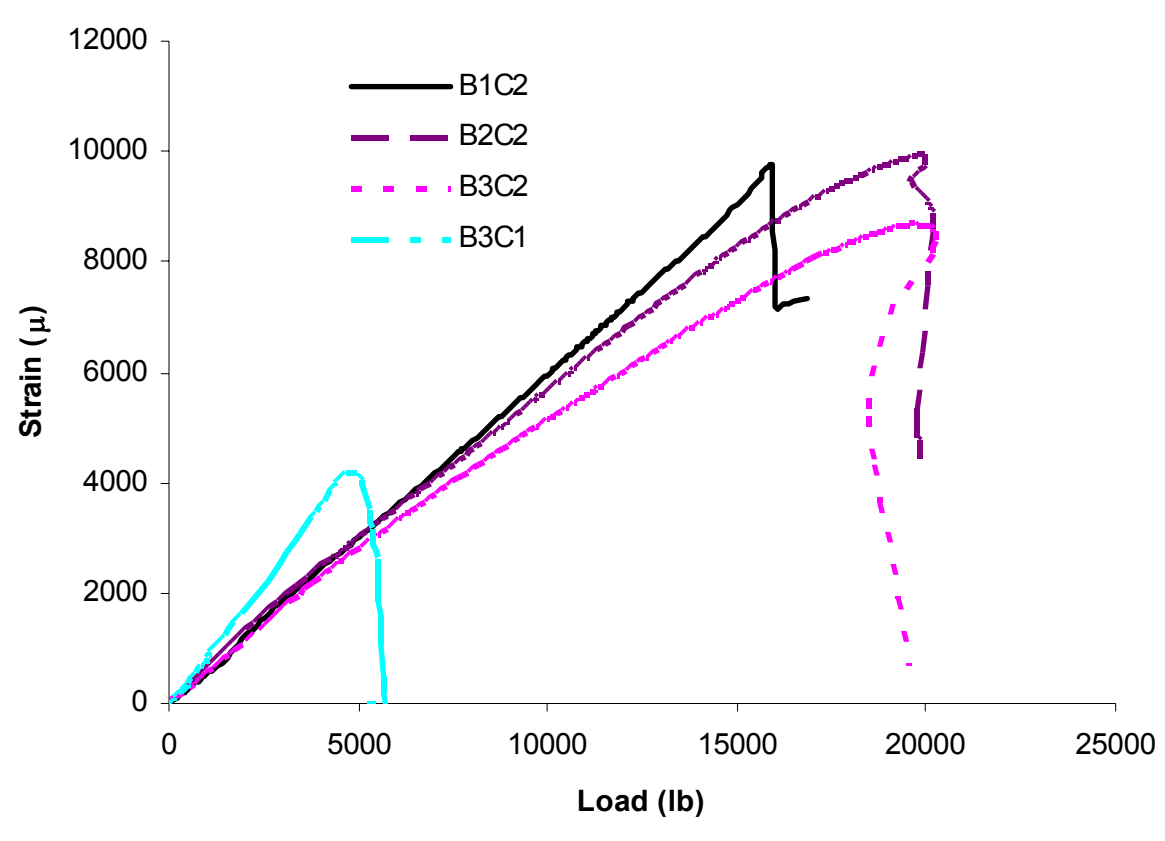

Figure 3.11 Strain-load curve for bare compression test

The maximum loads for specimens with distinct bonding layers are shown in Fig. 3.9, and the average value and standard deviation for six samples each are given in Table 3.6, which shows that the magnitudes of failure loads are in the same order as the number of bonding layers and core thickness; i.e., the specimen with three bonding layers is much stronger than that with one bonding layer, and the specimen with two core thickness is stronger than that with one core thickness, clearly showing that the bonding layer effect and core thickness play an important role on the failure load. Fig. 3.10 shows the loaddisplacement curve. Fig. 3.11 shows the transverse strain versus load curve for the sinusoidal panel. As the elastic pad is placed between the loading block and the specimen, this displacement does not represent the actual deformation of the specimen. However, from these figures we can conclude that the specimen exhibits an approximate linear behavior up to failure. 


\subsubsection{Stabilized Compression Test}

In this test, all three types of samples show the same failure mode. They all fail by crushing of the core panels. The sinusoidal wave panel fails first, followed by the crushing of the remaining components of the core, where the failure mode is shown in Fig. 3.12. No apparent damage can be observed prior to ultimate failure.

The failure loads for three specimens each are given in Table 3.7, which shows much higher values compared with what we obtained for the bare compression tests. Fig. 3.13 shows a typical load-displacement curve for the inside sinusoidal wave panels, and Fig. 3.14 gives the strain-load curve. Again we can see that the specimens follow a nearly linear behavior until failure occurs.

Table 3.7 Average value of failure load for stabilized compression test

\begin{tabular}{|c|c|c|c|}
\hline & B1C2 & B2C2 & B3C2 \\
\hline Average value (lb) & 34,965 & 36,660 & 39,840 \\
\hline Range (lb) & $33,435-36,500$ & $35,230-38,630$ & $35,550-45,320$ \\
\hline
\end{tabular}




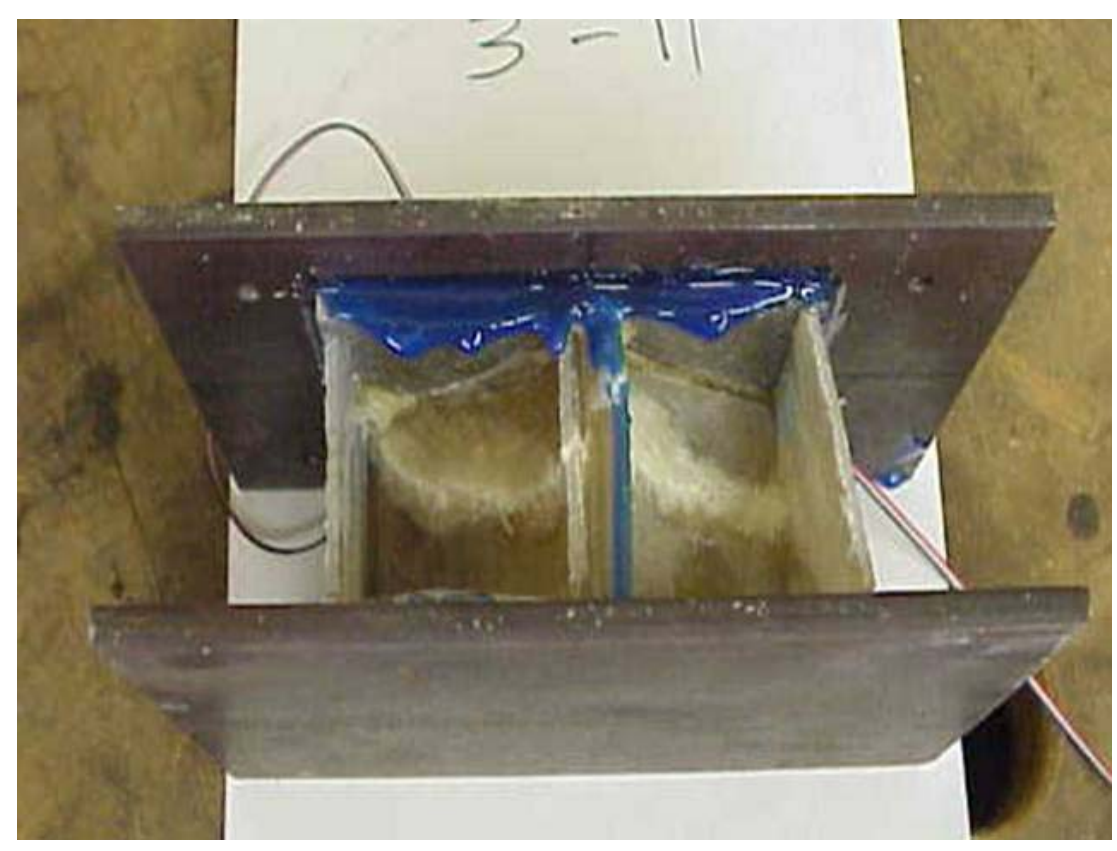

Figure 3.12 Stabilized compression test specimen

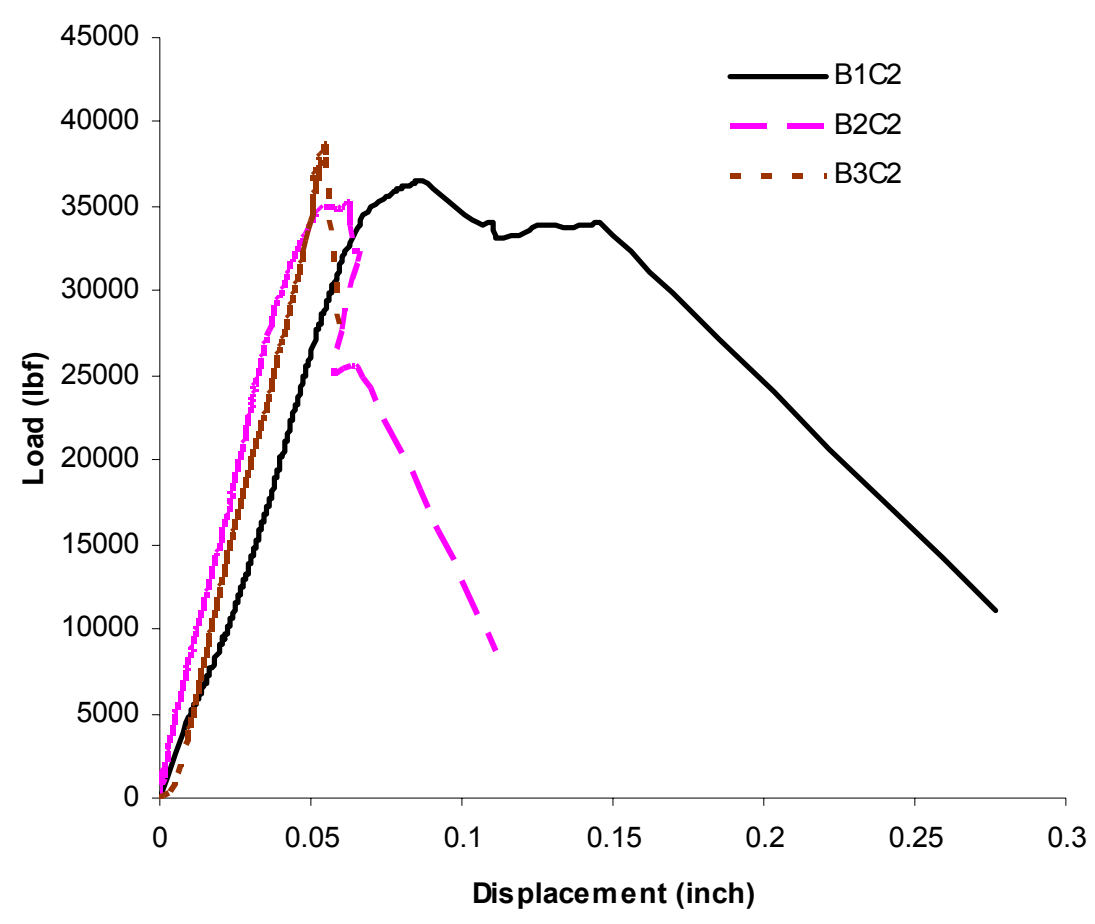

Figure 3.13 Load-displacement curve for stabilized compression test 


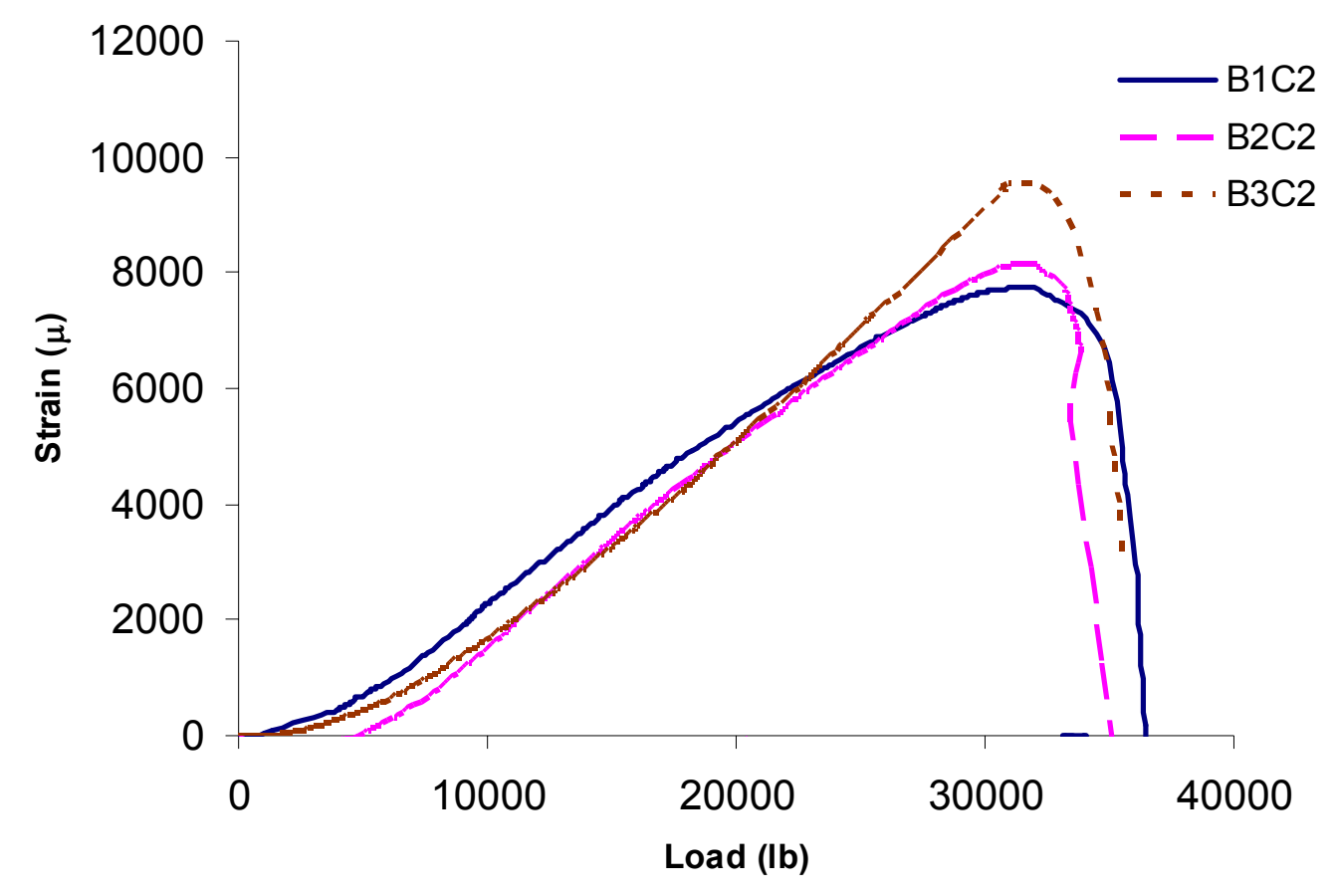

Figure 3.14 Strain-load curve for stabilized compression test

\subsubsection{Discussion of Experimental Results}

From previous studies, we can estimate the compressive strength for the ChSM to be about $22.2 \mathrm{ksi}$ for the present fiber volume fraction (Barbero et al., 1999). From the same test method as will be described in Chapter 5, the compressive strength is found to be 21.5 kips, as shown in Appendix B. Halpin and Kardos (1978) suggested a model to predict the compressive strength for ChSM using a pseudo-isotropic lamination method. Following his method, if the compression failure strains for the equivalent unidirectional composite is assumed to be $\varepsilon_{1 \mathrm{c}}=0.015$ and $\varepsilon_{2 \mathrm{c}}=0.006$, we can obtain the stress-strain curve to failure as given in Fig. 3.15. Therefore, it is reasonable to assume the compressive 
strength for ChSM material to be $21.5 \mathrm{ksi}$. The total in-plane area of the core walls with two core thickness is $2.13 \mathrm{in}^{2}$. Then, the nominal failure load can be calculated as

$$
F_{c}=f_{c} \times A_{c}=21.5 \times 2.13=45.8 \quad \text { kips }
$$

where $f_{c}$ is the compressive strength of ChSM and $A_{c}$ is the total in-plane area of the core walls.

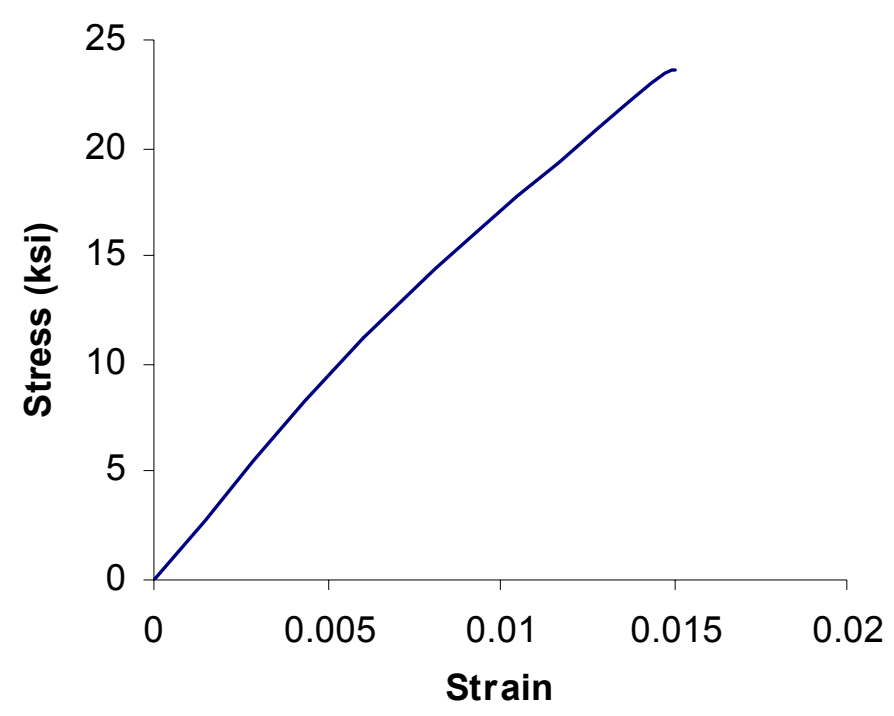

Figure 3.15 Stress-strain curve for ChSM

The stabilized test gives the failure load ranging from $33,435 \mathrm{lb}$ to $45,320 \mathrm{lb}$. If the unevenly distributed load effect is considered, we can conclude that the stabilized compression test results in a typical compression failure. For the bare compression test, the failure load is much lower than the nominal compressive load. This indicates that local buckling probably occurs before the structure gains its maximum compressive strength. Once the local buckling occurs, the buckled parts of the specimen lose their 
function and the compressive load is redistributed among the other parts. Finally the structure fails in compression or a combination of bending and compression.

The two types of tests resulted in two distinct failure modes. Buckling occurred for the bare compression test, while the stabilized compression test induced material compression failure. As a matter of interest, the two failure modes were the same as those reported by Zhang and Ashby (1992) under out-of-plane compression. As expected, the failure loads of stabilized compression tests are much higher than those for the bare compression tests.

\subsection{FE Analysis}

The same technique as described in Section 3.2 is used to carry out the FE analysis to correlate with test results. As discussed in the previous section, the stabilized compression test leads to compression failure, and the bare compression test is initiated by local buckling. Therefore, two types of analyses are carried out, namely static analysis and buckling analysis.

\subsubsection{Load-strain Curve}

A linear static analysis is used for the stabilized compression test and a buckling analysis is carried out for the bare compression test. As the bending of the side panels is observed in the bare compression test, geometric imperfection is included in the model for bare compression test to account for this deformation. The core-wall thickness is used as scale factor for geometric imperfection: $0.5 \mathrm{t}$ for the side panels. After extracting the $4^{\text {th }}$ eigenmode (Fig 3.16), the modified Riks method is used in the analysis (ABAQUS, 
2002). As the compressive load in the test is applied through a rigid loading block, the facesheet should displace downward at the same rate. Thus, Multiple Point Constraint (MPC) condition is used to allow the nodes in the same horizontal plane to move at the same displacement. Fig. 3.17 and Fig. 3.18 show comparisons of FE analysis results and test results for strain-load responses, showing good correlation between the two results.

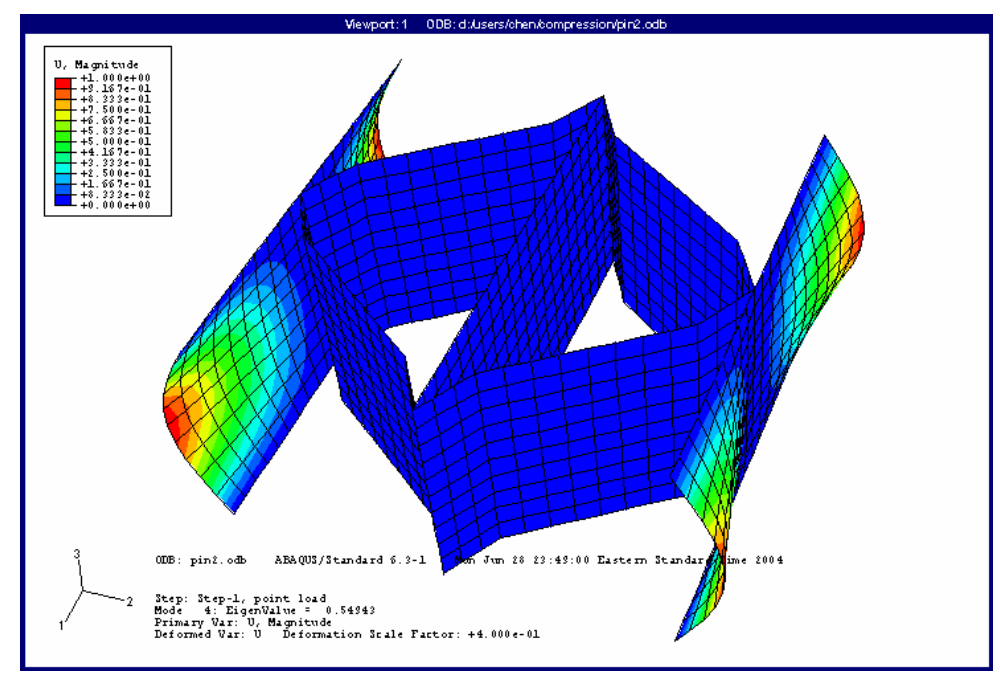

Figure 3.16 Imperfection mode

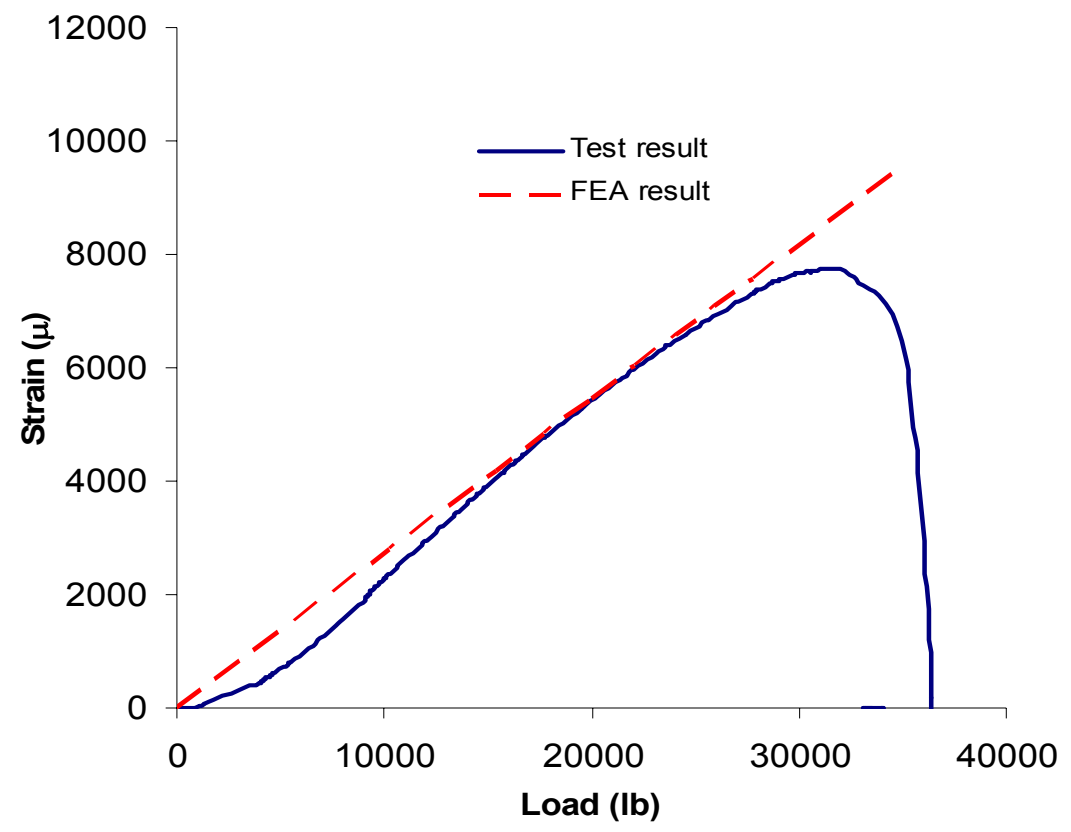

Figure 3.17 Load-strain curves for stabilized compression test 


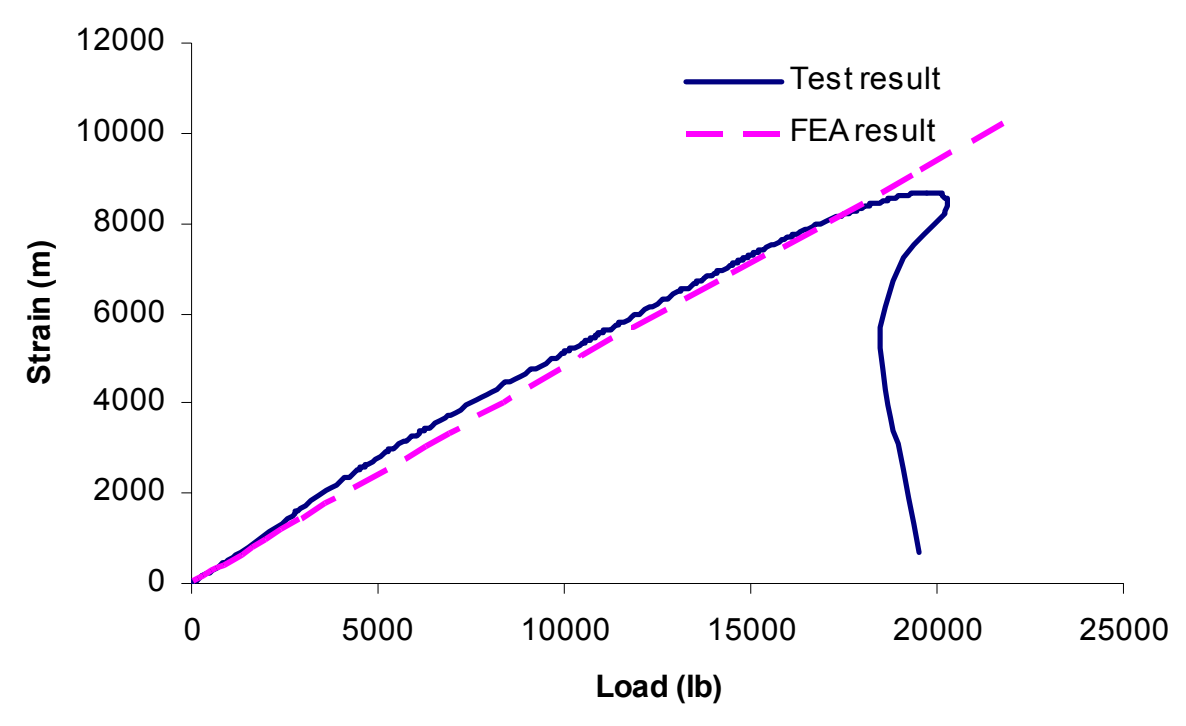

Figure 3.18 Load-strain curves for bare compression test

\subsubsection{Analysis Results and Discussion}

The FE results indicate that the buckling load is $30,368 \mathrm{lb}$ for the clamped condition and 10,821 $\mathrm{lb}$ for the hinged condition. The failure load of bare compression tests falls within this range, which indicates that the actual connection lies between simply supported and fully restrained conditions.

CER is dependent on the constrain element between the facesheet and core, such as the bonding layer thickness and core stiffness. If this coefficient can be determined, the local buckling strength can be computed. Therefore, a necessary step is to independently define the elastic restrain coefficient, which will be given in Section 3.5 through a cantilever plate test. 


\subsection{Determination of the Coefficient of Elastic Restraint}

As pointed out earlier, the facesheet and core are not rigidly connected, and therefore, CER is defined to quantify the degree of connectivity at the interface. To determine this coefficient, a testing method is developed in this section. The test setup is schematically shown in Fig. 3.19, with the core wall embedded into the facesheet. Fig. 3.20 displays a photograph of the test setup.

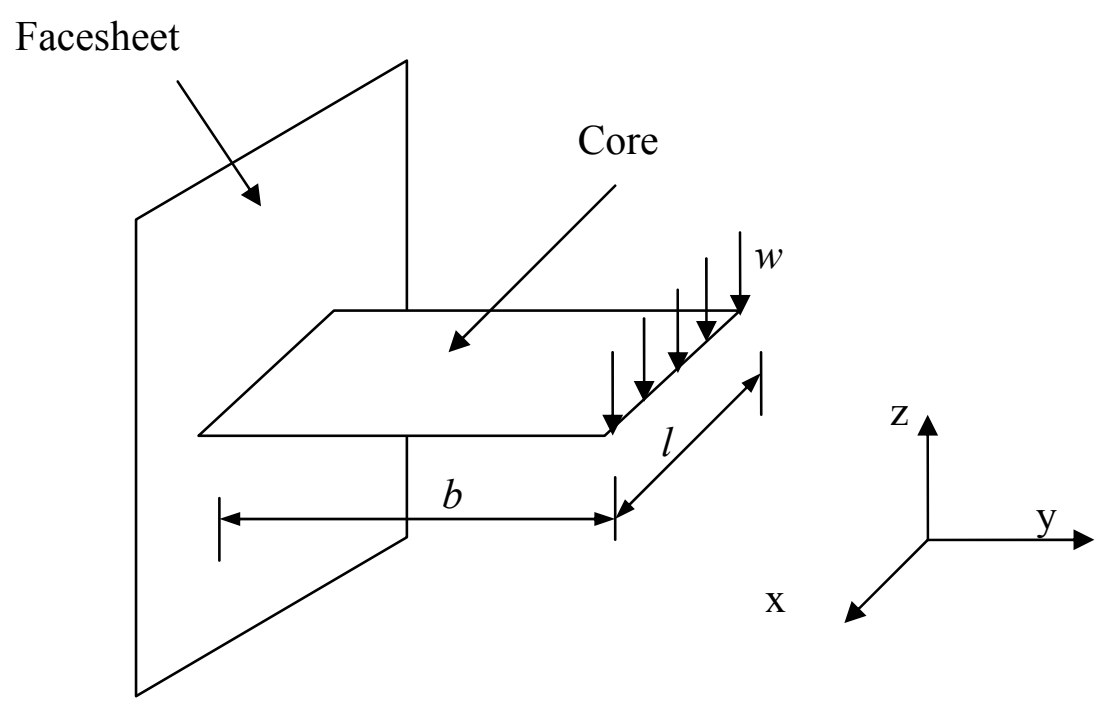

Figure 3.19 Test setup to determine the elastic restraint coefficient 


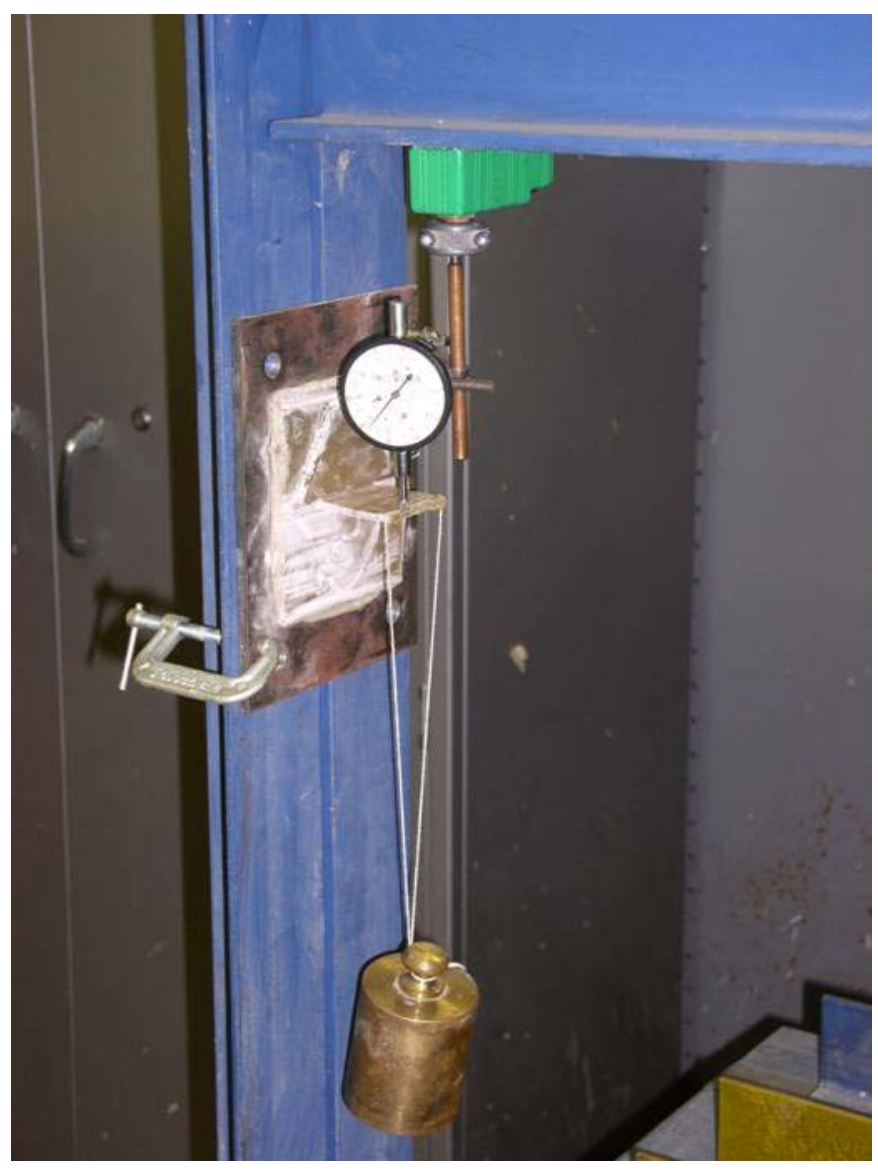

Figure 3.20 Photo of test setup

If the connection is rigid, considering the line load acting at the end of this cantilever plate and neglecting the shear deformation of the thin plate, the deflection at the end for rigid boundary condition is given as

$$
\Delta_{1}=\frac{w b^{3}}{3 D_{22}}
$$


where $w$ is the distributed line load acting at the end of the plate. However, as the core element is not rigidly connected, there is a rotation at the connection, which can be calculated as

$$
\bar{\varphi}=\frac{M}{\bar{\zeta}}=\frac{w b}{\bar{\zeta}}
$$

The relative deflection at the end of the plate corresponding only to this rotation is

$$
\Delta_{2}=\bar{\varphi} b
$$

where $b$ is the length of the panel, as shown in Fig. 3.19.

Then the total deflection becomes

$$
\Delta=\Delta_{1}+\Delta_{2}=\frac{w b^{3}}{3 D_{22}}+\bar{\varphi} b
$$

Following the same procedure, if $w$ is acting at the mid-span of the plate, the deflections at the end of the plate, for rigid connection and due to the relative rotation, can be respectively calculated as

$$
\Delta_{1}{ }^{\prime}=\frac{5 w b^{3}}{48 D_{22}}
$$




$$
\begin{aligned}
& \Delta_{2}{ }^{\prime}=\frac{w b}{2 \bar{\zeta}}=\frac{\bar{\varphi} b}{2} \\
& \Delta^{\prime}=\Delta_{1}{ }^{\prime}+\Delta_{2}{ }^{\prime}=\frac{5 w b^{3}}{48 D_{22}}+\frac{\bar{\varphi} b}{2}
\end{aligned}
$$

Solving simultaneously for Eq. (3.32) and Eq. (3.35), we obtain

$$
\bar{\varphi}=\frac{3.2 \Delta^{\prime}-\Delta}{0.6 b}
$$

Substituting Eq. (3.36) into Eq. (3.30), using Eq. (3.32), and based on the definition of $\zeta$ in Eq. (3.21), we can obtain the coefficient of restraint $\zeta$ through some simple transformations as

$$
\zeta=\frac{16 \Delta^{\prime}-5 \Delta}{12 \Delta-24 \Delta^{\prime}}
$$

Eq. (3.37) shows that this coefficient is only related to the two deflections, irrespective of the dimensions of the plate and the applied load. Thus, the accuracy of this testing method depends only on the measurement of tip displacements for the two load cases. We can test the validity of Eq. (3.37) by considering two extreme cases. If the connection is completely rigid, only the deflection corresponding to a rigid end is present as $\Delta=3.2 \Delta^{\prime}$, resulting in $\zeta=0$. While for a hinged connection, the flexural deflection is negligible compared with the tip displacement, due to the hinge rotation, which becomes $\Delta=2 \Delta^{\prime}$, 
and results in $\zeta=\infty$. These results correspond to the range of values defined previously for $\zeta$.

Table 3.8 Average value of CER with $b=2$ "'

\begin{tabular}{|c|c|c|c|}
\hline & One bonding layer & Two bonding layers & Three bonding layers \\
\hline Average value & 0.84 & 0.41 & 0.29 \\
\hline Range & $0.81-0.87$ & $0.40-0.41$ & $0.27-0.33$ \\
\hline
\end{tabular}

Tests were carried out for the three cases of distinct bonding layer thickness. The specimens were cut from the same samples as used in the compression tests described above, with $l$ and $b$ (Fig. 3.19) both equal to 2 ". A standard weight of $2 \mathrm{~kg}$ was used to apply the load both at the end and mid-span of the plate, and a dial gage with a precision of 0.0001 " was used to measure the displacement at the end of the plate. The test results are listed in Table 3.8 . 


\subsection{Comparisons of Test Results with Analytical and FE Predictions}

Table 3.9 Comparison of analytical, FE and test results for buckling load

\begin{tabular}{|c|c|c|c|}
\hline & B1C2 & B2C2 & B3C2 \\
\hline Analytical result (lb) & 16,780 & 20,451 & 22,438 \\
\hline FE result (lb) & 16,358 & 19,918 & 21,776 \\
\hline Test result (lb) & 16,770 & 21,010 & 22,900 \\
\hline
\end{tabular}

Using the CER value obtained from the cantilever plate test described above into Eq. (3.24), we can predict the buckling load. Two sets of $p$ and $q$ are used, one from the analytical solution and the other from the FE analysis, and their corresponding critical loads for hinged and fixed conditions, as listed in Table 3.2. The results are summarized in Table 3.9, showing good correlations of test results with analytical and FE predictions.

\subsection{Parametric Study}

In practice, it is common to vary the core height to meet the requirement for the panel depth. Using the analytical model derived and the CER obtained, we can carry out a parametric study by varying the core height for the specimen studied. The critical buckling stress vs. core height curve is illustrated in Fig. 3.21 for a=4". From which it can be seen that the buckling stress is quite sensitive to variation of cell height up to about 4", and within this range there is a notable difference among the buckling stresses for different number of bonding layers. The buckling stress decreases as the core height increases, and the stress reaches nearly a plateau when the core height is higher than 8 ". Beyond this limit point the bonding layer thickness does not affect the result much. The 
reason for this behavior is that when the aspect ratio of height over width is less than one, the number of the half waves parallel to the loading direction is one, and therefore, the boundary condition will affect the buckling load. But as core height increases, more half waves along the loaded direction will result, and in this case, the buckling load will be determined by the wave-length in between the two loaded edges. As a result, the boundary condition does not affect the buckling load much. If we keep the height fixed, we can find the relationship between the buckling stress and length of the flat panel, as shown in Fig. 3.22. The buckling stress increases as the length increases, and it is anticipated that when the length increases infinity, i.e., the aspect ratio approaches zero, the plate will not buckle. Clearly, Fig. 3.21 and 3.22 also illustrates the relationship between the critical buckling stress and the aspect ratio of the core wall.

Multiply the buckling stress by the total core wall length, the buckling load vs. core height curve is given in Fig. 3.23 for $\mathrm{a}=4$ " case for different bonding layers. For a given height, we can easily find the buckling load for a single cell from these curves. 


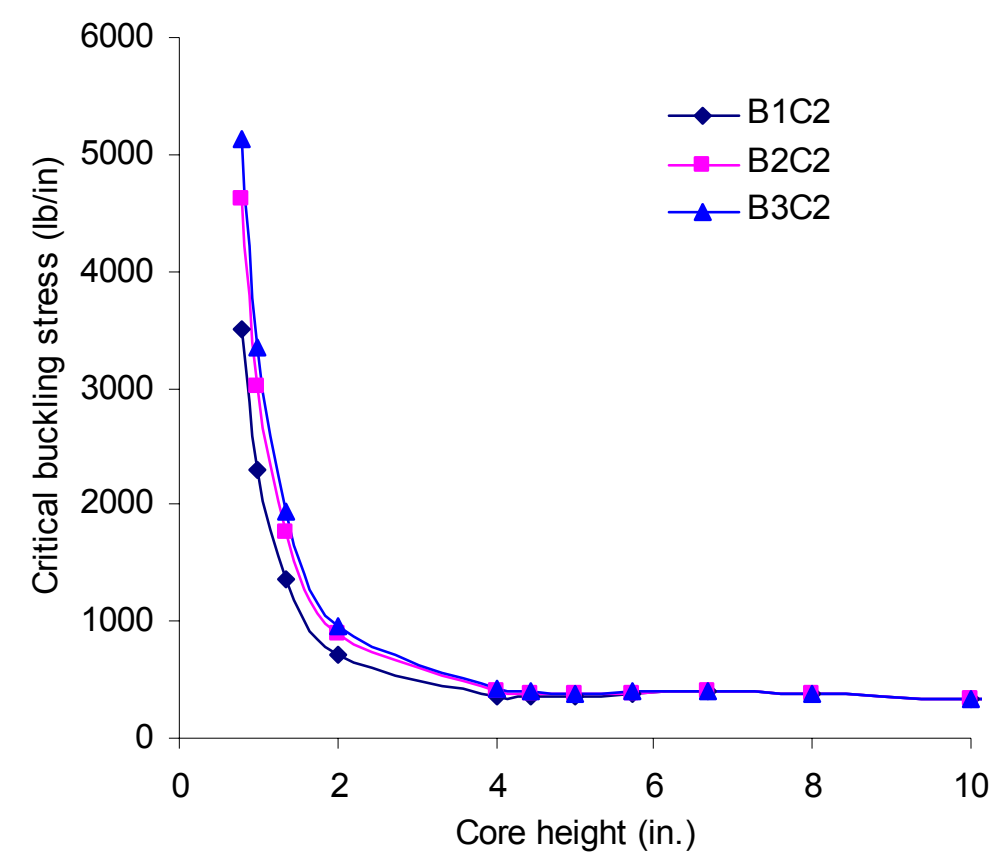

Figure 3.21 Critical buckling stress vs. core height

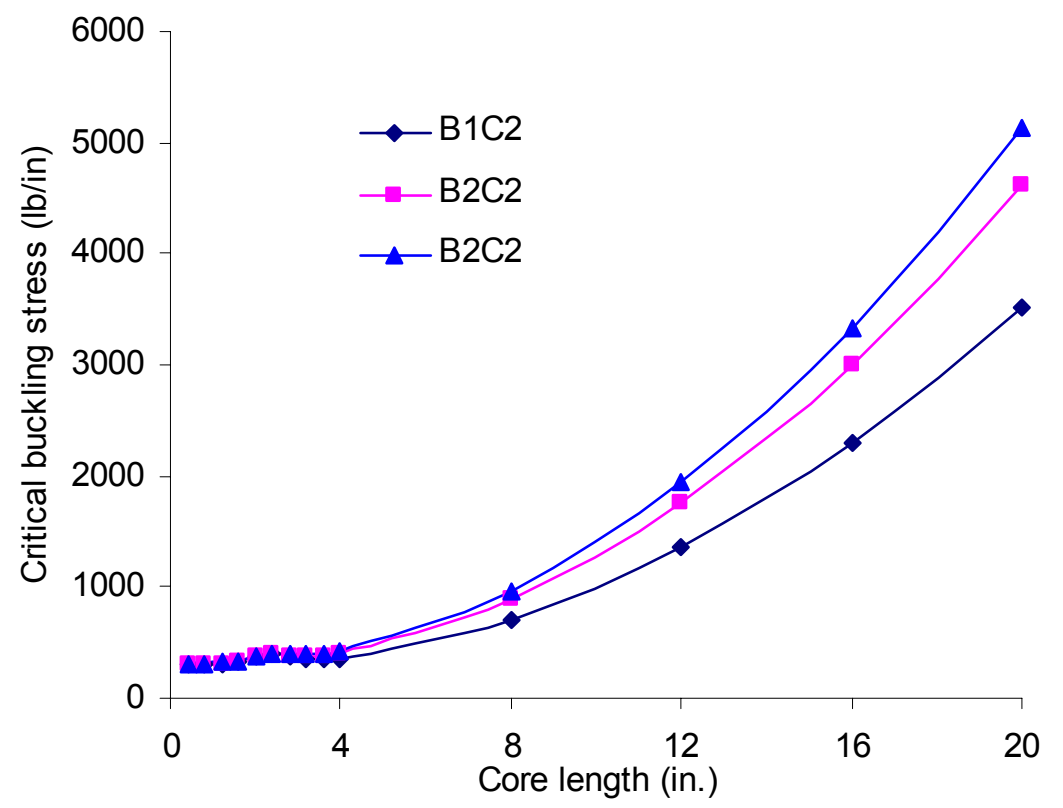

Figure 3.22 Critical buckling stress vs. length of flat panel 


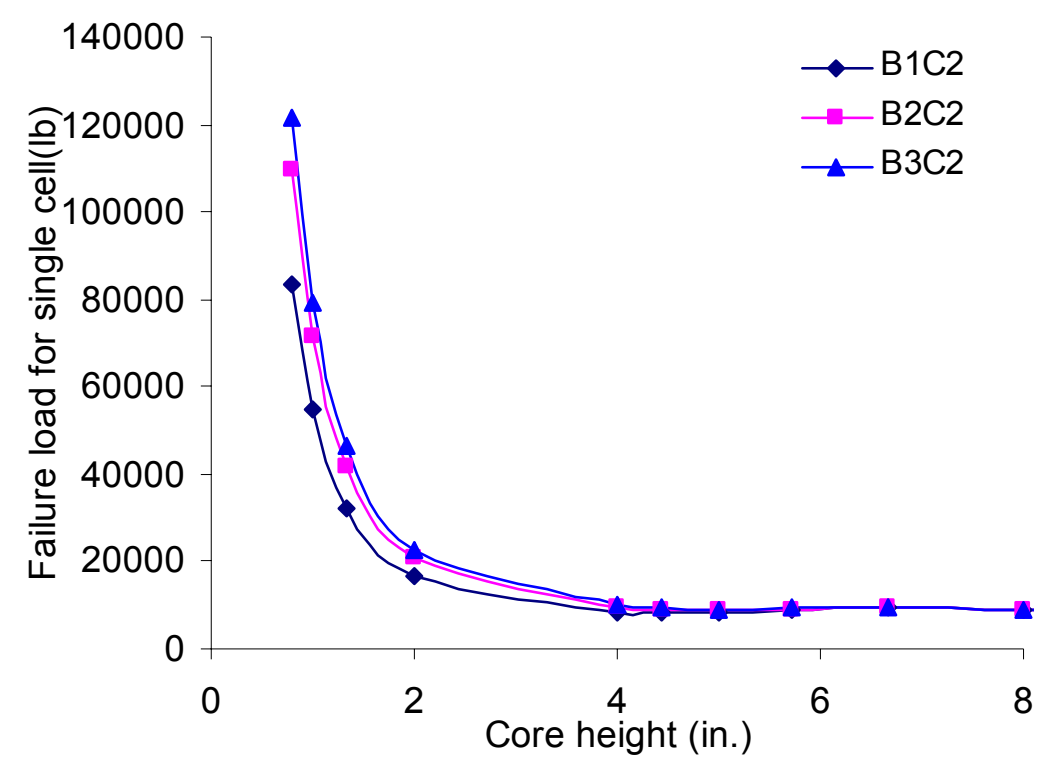

Figure 3.23 Buckling load vs. core height

\subsection{Design Equations}

Design equations can be developed based on the analytical model derived above.

Only two core thickness case, i.e., $t=0.09$ ”, which is most commonly used, is considered, while other core thickness can be constructed following the same manner.

Considering buckling failure, the three curves shown in Fig. 3.23 can be fitted using the following equation

$$
F=A_{1} e^{\left(-h / t_{1}\right)}+A_{2} e^{\left(-h / t_{2}\right)}+F_{0}
$$


The parameters corresponding to each bonding layer are listed in Table 3.10. It is noted that Eq. (3.38) gives the failure load for a single cell, and if it is divided by the inplane area for a single cell, which is $4 \times 4=16 \mathrm{in}^{2}$ for this case, the buckling compressive strength can be obtained.

Either Eq. (3.1) or the stabilized compression test can be used to find the failure load corresponding to core crushing, where the average value of $F_{c}=37,000 \mathrm{lb}$ from the test results is adopted herein. Based on the failure modes of core crushing and buckling, we can propose a design equation as shown in Fig. 3.24, where $\mathrm{h}_{\mathrm{T}}$ is the height where the failure mode transits from core crushing to core buckling, as listed in Table 3.11.

Table 3.10 Parameters for design equation

\begin{tabular}{|c|c|c|c|c|c|}
\hline & $\mathrm{A}_{1}$ & $\mathrm{t}_{1}$ & $\mathrm{~A}_{2}$ & $\mathrm{t}_{2}$ & $\mathrm{~F}_{0}$ \\
\hline One bonding layer & 957,515 & 0.2363 & 124,742 & 0.7464 & 8,081 \\
\hline Two bonding layers & 87,639 & 1.0105 & 95,4711 & 0.2917 & 8,136 \\
\hline Three bonding layers & 103,8189 & 0.2985 & 88,384 & 1.0765 & 8,152 \\
\hline
\end{tabular}

Table 3.11 Transition height

\begin{tabular}{|c|c|c|c|}
\hline & One bonding layer & Two bonding layer & Three bonding layer \\
\hline $\mathrm{h}_{\mathrm{T}}$ & $1.25 ”$ & $1.42 \%$ & $1.5 \%$ \\
\hline
\end{tabular}




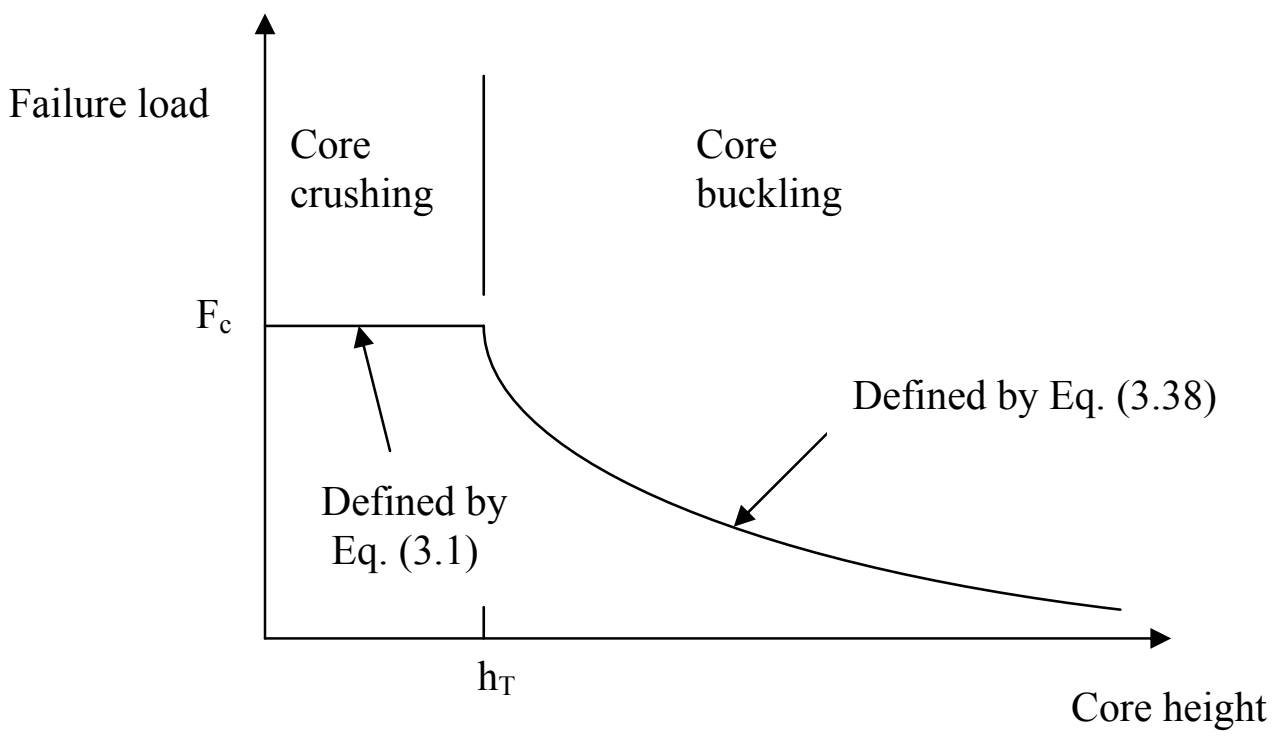

Figure 3.24 Design diagram

\subsection{Concluding Remarks}

Two analytical models, corresponding to pure compression and elastic buckling failure, respectively, are provided for panels subject to out-of-plane compression. A combined analytical and experimental study of elastic buckling analysis is given for FRP panels with elastic restraint at the loaded edges. By solving a transcendental equation, the critical compression buckling stresses are obtained. An elastic restrain coefficient is employed to quantify the elastic restraint effect, namely, the bonding layer effect. Buckling loads are calculated in terms of the elastic restraint coefficient. The analytical predictions are verified by FE analysis. The compression test is carried out to study the behavior of sandwich panels under out-of-plane compression. A cantilever plate test is conducted to capture the coefficient of elastic restraint. Both the closed form solution and 
FE analysis is used to predict the buckling load for the given test samples, and the results are in good correlation. A parametric study is carried out to study the aspect ratio effect on the buckling load. Based on the study shown in this chapter, the following conclusions can be drawn:

1. The closed form solution derived in this chapter can predict the buckling strength of a plate with partially restrained loaded edges. Unlike existing solutions for eigenvalue analysis, where the number of half waves should be predefined when calculating the buckling load, this solution can give the minimum buckling load and the corresponding number of half waves. The accuracy is verified by FE analysis and experimental results.

2. Typically there are two failure modes for HFPR core under out-planecompression, buckling and pure compression failure. The buckling load is sensitive to the bonding layer effect. Specimens with three bonding layers fail at a higher load than those with one bonding layer. While for pure compression failure, the failure loads are not affected much by the number of bonding layers.

3. Bonding layer effect can be interpreted through a coefficient of elastic restraint (CER). It plays an important role on the buckling behavior. However, rigid connection is commonly used in the analysis of sandwich structures, corresponding to $\zeta=0$ in this study. It is shown that a significant error may occur if the aspect ratio is within a certain limit.

4. CER can be predicted using the testing method provided. Only two deflections are required to calculate this coefficient. Thus, the accuracy is increased. This method 
together with interface shear test and interface tension test can be act as the criteria to define the bonding quality of a given connection.

5. A parametric study is carried out by varying the core height. The result indicates that, if the core height is relatively low, there is a notable difference of the buckling stress for different number of bonding layers. The buckling stress decreases as the core height increases and reaches nearly a constant value once the core height reaches a certain limit. The buckling load is no longer sensitive to the bonding layer effect at this stage.

6. Practical design equations are provided to calculate the compressive strength, which can be easily implemented.

Bonding layer effect not only affects the buckling load, but also influences the behavior of the sandwich panels under out-of-plane shear. This will be presented in the following chapter. The method described in this chapter can be further applied to other structures with elastic restraint at the loaded edges, like the web buckling in the steel girder. 


\section{CHAPTER 4}

\section{OUT-OF-PLANE SHEAR}

\subsection{Introduction}

A combined analytical and experimental study of FRP sandwich panel under outof-plane shear is presented in this chapter. Analytical models, including delamination considering skin effect, shear crushing, and shear buckling are provided. Two factors are addressed that contribute to the skin effect: shear warping and bending warping. A closed-form solution, based on proper description of displacement field at the interface, is derived considering shear warping. The accuracy of this method is verified by FE results. The FE model is then applied to study bending warping effect. The stiffness and the stress distribution subject to skin effect are presented. Critical parts are identified and suggestions for future design considerations are given. Based on the stress distribution, design formulas for delamination and shear failure are presented. Rayleigh-Ritz method is employed to study the shear buckling of core panels with two sides elastically restrained. Four-point bending tests were carried out according to ASTM standards to study shear strength and shear stiffness of the core materials. The number of these bonding layers and core thickness were varied to study their effect on strength. Two types 
of beam samples were manufactured by orienting the sinusoidal wave: (1) along the length ("longitudinal"), and (2) along the width ("transverse"). Different failure modes were observed for different type of specimen. Design equations are used to predict the failure load due to different failure modes and good correlations are obtained.

\subsection{Analytical Model including Skin Effect}

\subsubsection{Origin of Skin Effect}

As shown in the literature review, the only work that can be found on the study of shear stiffness for this sinusoidal core was by Xu, Qiao and Davalos (2001), where the lower bound of the transverse shear stiffness was provided neglecting skin effect. There is no study on accurate description of the transverse shear stiffness and stress distribution, partly due to the complex displacement field, especially for curved panels, such as the sinusoidal panel in this study. Chen and Davalos (2004) pointed out that the displacement field in cell walls for sandwich core can be described by two distinct modes: 1) directly at the face-core interface, if facesheet is assumed to be rigid, which is reasonable considering the stiffness ratio between the facesheet and core, it is defined by displacement compatibility, where strain transformation can be used to find the relationship between local and global strain; and 2) at a position sufficiently far away from the interface, i.e., such as at the mid-depth where the effect of rigid facesheet dissipates, it is defined by force equilibrium. Therefore, the purpose of the analysis is to find a displacement field that can accurately describe these two distinct modes and the transition field in between. In order to achieve this, the displacement field at the interface has to be properly described first. A basic assumption for all previous studies on the 
equivalent properties of sandwich core is that the cell walls predominantly carry load through membrane strain, and that the bending forces in the cell walls are neglected. However, the bending effect should play a role when defining the shear stiffness and shear distribution, especially when the core height is low. Therefore, we believe that shear and bending warping effects are better descriptors of these phenomena, where shear warping corresponds to the assumption adopted in the previous studies, and the bending warping describes the additional bending effect offered by the skin. Furthermore, it is found out that shear warping corresponds to cases with hinge connection between facesheet and core, and when both warping effects are considered, it corresponds to a rigid connection. The actual cases usually lie in between these two conditions. Detailed descriptions of skin effect are as follows.

\subsubsection{Skin Effect}

A unit cell of honeycomb sandwich panel and its dimensions is shown in Fig. 4.1 and Fig. 4.2, respectively. Two factors may contribute to skin effect: shear and bending warping. 


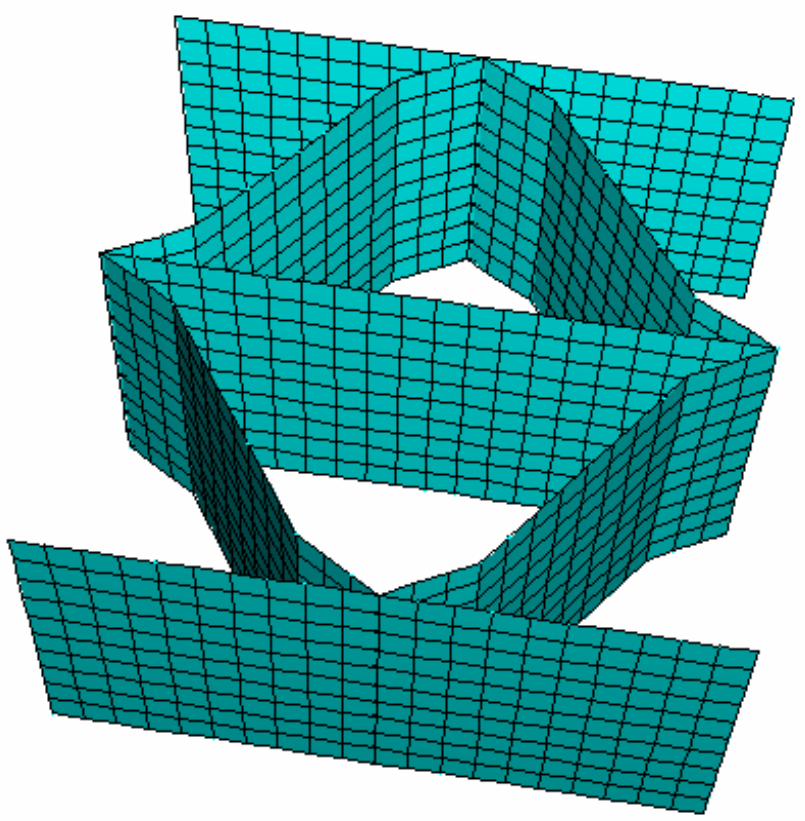

Figure 4.1 Unit cell

Flat panel

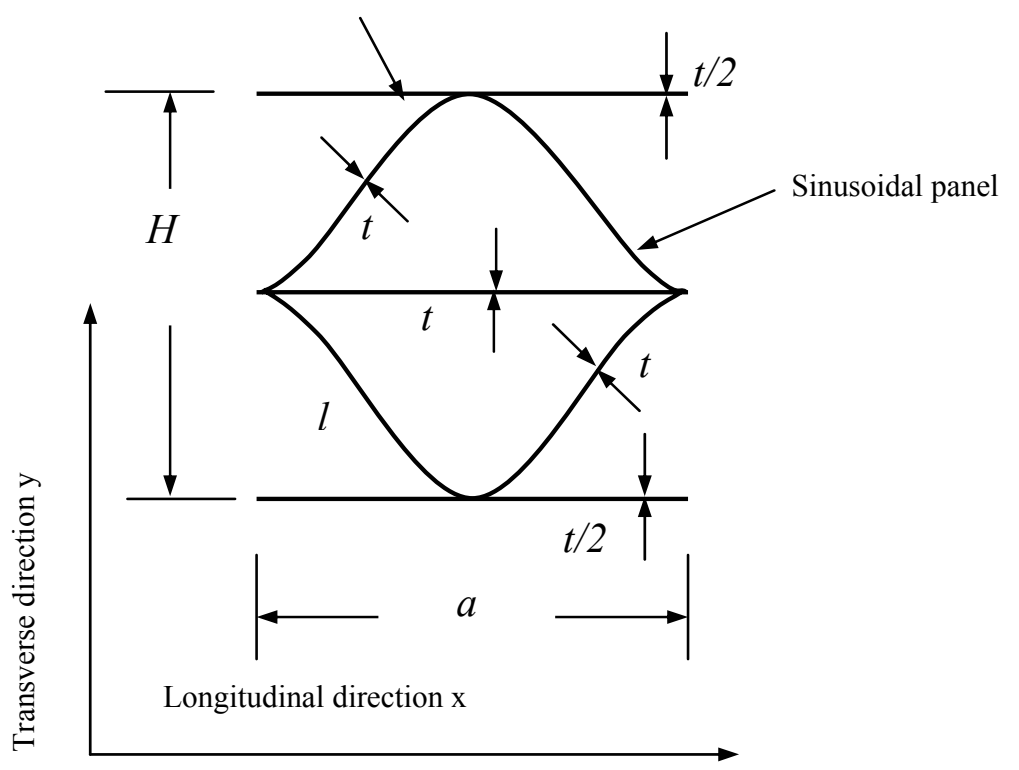

Figure 4.2 Dimensions of a unit cell 


\subsubsection{Shear Warping}

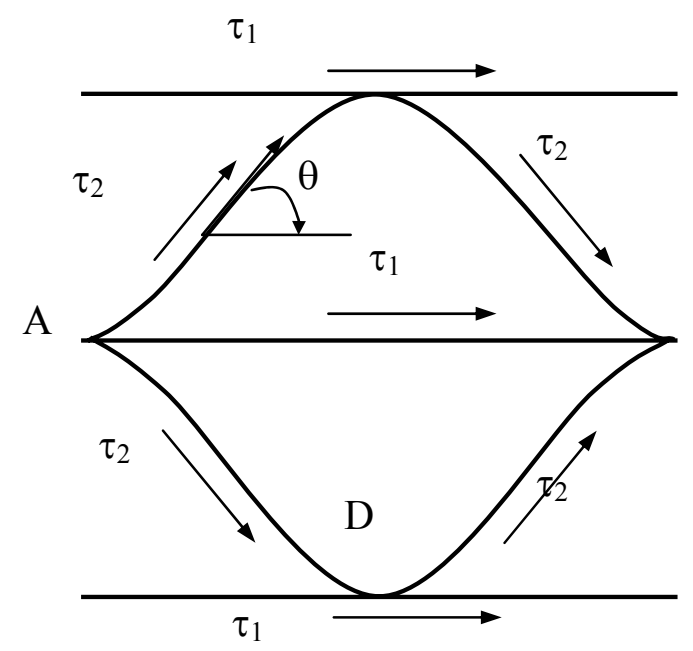

Figure 4.3 Shear flow in the unit cell
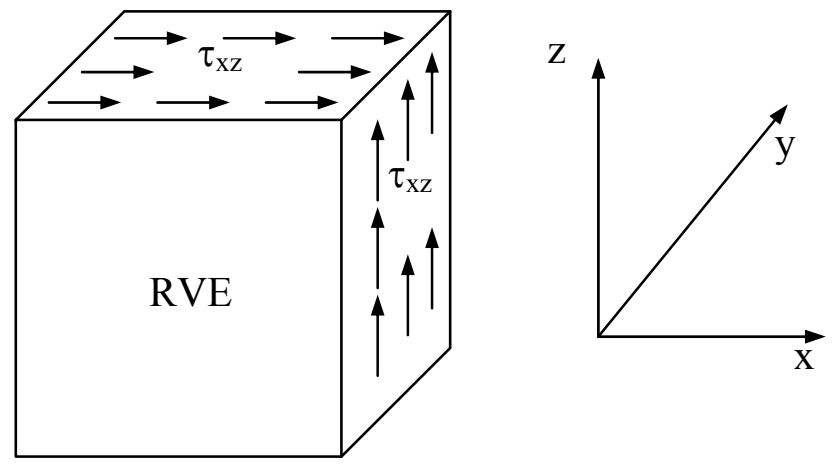

Figure 4.4 Shear flow in the RVE 


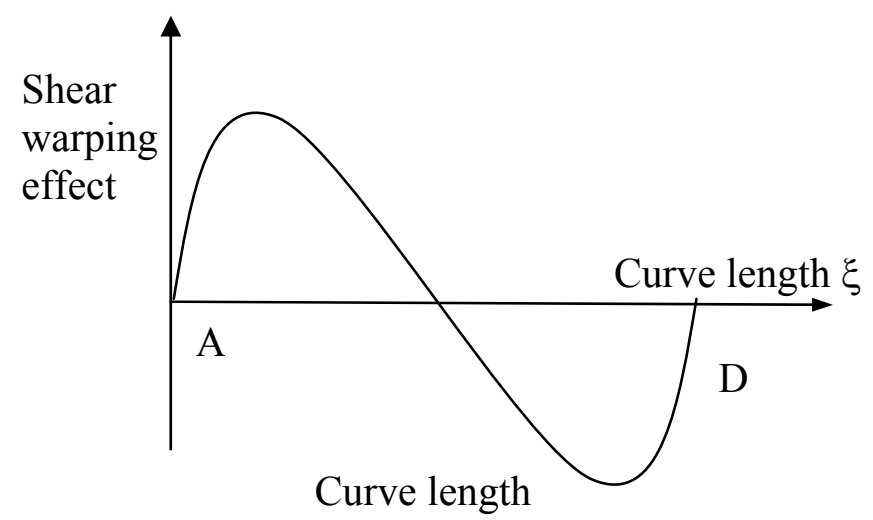

Figure 4.5 Shear warping

(Plan view)

The resulting distributed shear flow for a typical cell and its Representative Volume Element (RVE) are shown in Fig. 4.3 and Fig. 4.4. The equilibrium equation and compatibility condition for a longitudinal wave configuration without considering skin effect can be written as

$$
\begin{aligned}
& 4 t \int_{0}^{l} \tau_{2} \cos \theta d s+2 \operatorname{ta} \tau_{1}=H a \tau_{x z} \\
& 2 \int_{0}^{l} \tau_{2} d s / G_{12}=\tau_{1} a / G_{12}
\end{aligned}
$$

where $2 \int_{0}^{l} \cos \theta d s=a, G_{12}$ is the material shear modulus, $l$ is the curved panel length, and $t, a$, and $H$ are defined in Fig. 4.2. Solving for Eqs. (4.1) and (4.2), we have 


$$
\begin{aligned}
& \tau_{1}=\frac{H \int_{0}^{l} d s}{a t+2 t \int_{0}^{l} d s} \tau_{x z} \\
& \tau_{2}=\frac{H}{2 t+4 t \int_{0}^{l} d s / a} \tau_{x z}
\end{aligned}
$$

From Eqs. (4.3) and (4.4), we have

$$
\tau_{1} / \tau_{2}=2 \int_{0}^{l} d s / a
$$

and correspondingly, we can obtain

$$
\gamma_{1} / \gamma_{2}=2 \int_{0}^{l} d s / a
$$

where $\gamma_{1}$ and $\gamma_{2}$ are the shear strains in the flat and curved panels, respectively. Apparently, the flat panel will deform along a straight line, while the curved panel deforms along a curved shape as shown in Fig. 4.5 (only half of the top curve is shown). However, in most practical cases, the face and the core are constrained so that they remain essentially plane during deformation. Therefore, to compensate the deformation shown in Fig. 4.5, the shear warping occurs at the top and bottom of a curved panel. The expression of shear warping will be given in Section 4.2.4. 


\subsubsection{Bending Warping}

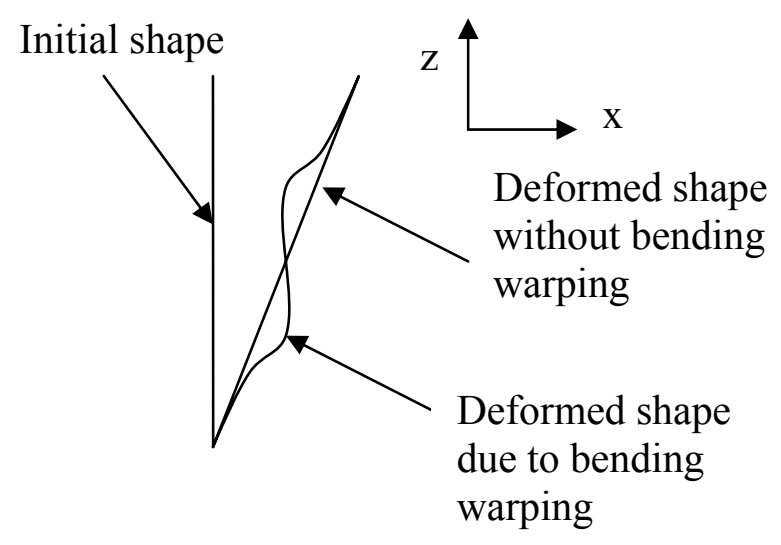

Figure 4.6 Bending warping

(Elevation view)

Pure shear strain in the curved wall will induce a displacement in the $\mathrm{x}$ direction, as shown in Fig. 4.6. However, if we assume core-facesheet is rigidly connected, the rotation at the top and bottom of the core is constrained, resulting in a deformed shape as shown in Fig. 4.6. This phenomenon can be termed as bending warping. Apparently, an additional moment at both top and bottom will result due to this effect.

It should be noted that, although both effects are local, they can significantly affect the stress distribution at both the top and bottom of the core, i.e., the interfacial stresses, as discussed below. 


\subsubsection{Theoretical Analysis}

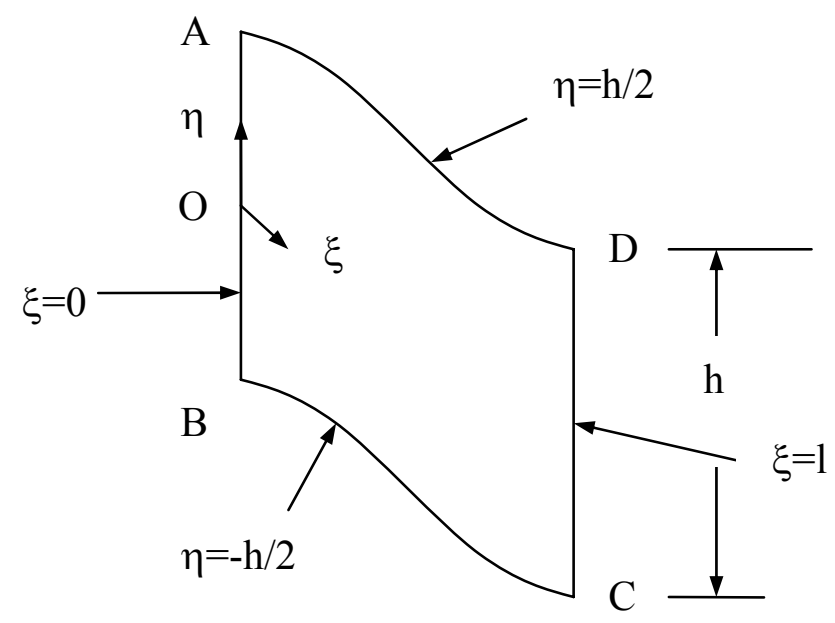

\section{Figure 4.7 Model used for theoretical analysis}

Consider the element ABCD in Fig. 4.7, which is cut from the unit cell shown in

Fig. 4.2, subject to a shear strain $\gamma$. The equilibrium equation for the stresses acting on the $\xi \eta$ plane in the absence of body forces is

$$
\begin{aligned}
& \partial \sigma_{\xi} / \partial \xi+\partial \sigma_{\xi \eta} / \partial \eta=0 \\
& \partial \sigma_{\xi \eta} / \partial \xi+\partial \sigma_{\eta} / \partial \eta=0
\end{aligned}
$$

Considering the stress-strain relationship, Chen and Davalos (2004) further reduced Eqs.

(4.7) and (4.8) into the form (see Appendix C) 


$$
G\left(\partial^{2} v / \partial \xi^{2}\right)+E^{\prime}\left(\partial^{2} v / \partial \eta^{2}\right)=0
$$

where $E^{\prime}=E /\left(1-v^{2}\right)$. The stress components can be defined as

$$
\begin{gathered}
\sigma_{\eta}=E^{\prime}(\partial v / \partial \eta) \\
\tau_{\xi \eta}=G(\partial v / \partial \xi) \\
\sigma_{\xi}=v E^{\prime}(\partial v / \partial \eta)
\end{gathered}
$$

Eqs. (4.9)-(4.12) act as the basis for this analytical study. The boundary conditions considering shear warping are:

$$
\begin{aligned}
& v(0, \eta)=v(l, \eta)=0 \\
& v(\xi, \eta)=v(\xi,-\eta) \\
& v(\xi, h / 2)=\varphi(\xi)
\end{aligned}
$$

where $l$ is the curved panel length and $h$ is the height. Then, $\varphi(\xi)$, caused by shear warping as shown in Fig. 4.5, can be defined as

$$
\varphi(\xi)=\gamma_{1} x(\xi)-\gamma_{2} s(\xi)
$$

where, $x(\xi)=$ length of flat panel, and $s(\xi)=$ length of curved panel. 
The solution of Eq. (4.9) can be described using Fourier Series as

$$
v(\xi, \eta)=\sum_{n=1}^{\infty}\left[\frac{2 / l}{\cosh \left(\frac{n \pi h}{2 l \mu}\right)} \varphi_{n} \cosh \left(\frac{n \pi \eta}{l \mu}\right) \sin \left(\frac{n \pi \xi}{l}\right)\right]
$$

where

$$
\begin{aligned}
& \mu=\sqrt{E^{\prime} / G} \\
& \varphi_{n}=\int_{0}^{l} \varphi(\xi) \sin \frac{n \pi \xi}{l} d \xi
\end{aligned}
$$

The normal stress $\sigma_{\eta}$ can be obtained using Eq. (4.10) as

$$
\sigma_{\eta}(\xi, \eta)=E^{\prime}(\partial v / \partial \eta)=E^{\prime} \sum_{n=1}^{\infty}\left[\frac{2 n \pi}{l^{2} \mu \cosh \left(\frac{n \pi h}{2 l \mu}\right)} \varphi_{n} \sinh \left(\frac{n \pi \eta}{l \mu}\right) \sin \left(\frac{n \pi \xi}{l}\right)\right]
$$

Eq. (4.11) gives

$$
\tau(\xi, \eta)=G(\partial v / \partial \xi)=G \sum_{n=1}^{\infty}\left[\frac{2 n \pi}{l^{2} \cosh \left(\frac{n \pi h}{2 l \mu}\right)} \varphi_{n} \sinh \left(\frac{n \pi \eta}{l \mu}\right) \cos \left(\frac{n \pi \xi}{l}\right)\right]
$$

The normal stress $\sigma_{\xi}$ can be obtained using Eq. (4.12). 
Next, the total strain energy is defined as

$$
U=\int \frac{\tau^{2}}{2 G_{12}} d V+\int \frac{\sigma^{2}}{2 E} d V
$$

in order to obtain equivalent shear modulus $G_{x z}$

$$
G_{x z}=\frac{2 U}{V \gamma^{2}}
$$

where $U$ is total strain energy, $V$ is the volume corresponding to the RVE, $\gamma$ is the shear strain applied to the structure, which is equal to $\gamma_{l}$ in value. The above equations can be incorporated into any mathematical software such as MATHCAD. 


\subsubsection{Description of FE Model}

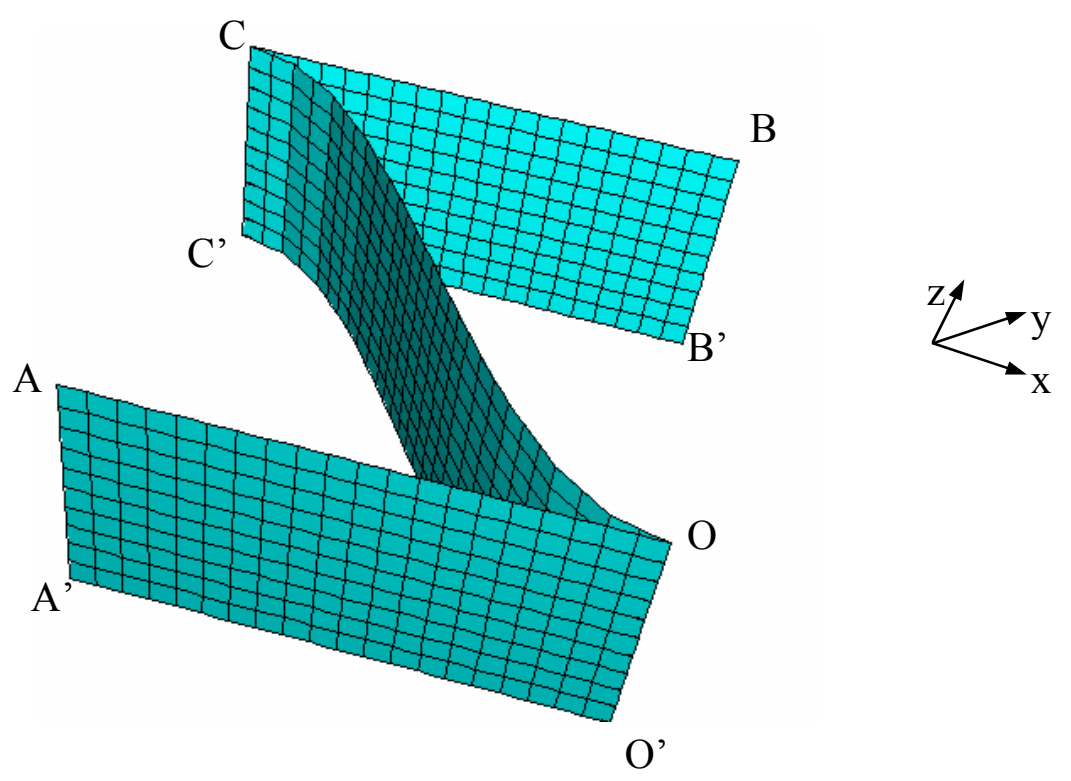

Figure 4.8 Model used for FE analysis

FE method is employed to verify the analytical model derived in Section 4.2.4. A unit cell of honeycomb sandwich panel and its dimensions is shown in Fig. 4.1. Due to the symmetric structure, we can further reduce the cell into a quarter cell, as shown in Fig. 4.8. This quarter cell will be used in the FE analysis. Based on symmetry, the thickness is $t / 2$ for the flat panels and $t$ for the sinusoidal panel. The height of the core is half of the unit cell dimensions. The dimensions and properties of the core materials are listed in Table 4.1.

In the FE analysis, all the nodes at the top face translate at a uniform displacement in the $\mathrm{x}$ direction. The shear force can be computed by summing up the reaction force along the $\mathrm{x}$ direction for all the nodes at the top. Thus, the equivalent shear stiffness $G_{x z}$ and shear stress distribution can be obtained. The boundary conditions are listed in Table 
4.2, where $\mathrm{CB} / \mathrm{AO}$ is defined according to the restraint condition assumed. In particular, free, pinned and fixed boundary conditions correspond to free, hinge and rigid connections.

Table 4.1 Properties of core mat

\begin{tabular}{|c|c|c|c|c|}
\hline $\mathrm{H}$ (in.) & $\mathrm{a}$ (in.) & $\mathrm{t}$ (in.) & $\mathrm{E}\left(\mathrm{x} 10^{6} \mathrm{psi}\right)$ & $\mathrm{v}$ \\
\hline 4 & 4 & 0.09 & 1.71 & 0.402 \\
\hline
\end{tabular}

Table 4.2 Boundary conditions of FE model

\begin{tabular}{|c|c|c|c|}
\hline & $\mathrm{u}_{\mathrm{x}}$ & $\mathrm{u}_{\mathrm{y}}$ & $\mathrm{u}_{\mathrm{z}}$ \\
\hline $\mathrm{OO}^{\prime} / \mathrm{BB}^{\prime}$ & Free & 0 & 0 \\
\hline $\mathrm{CC}^{\prime} / \mathrm{AA}^{\prime}$ & Free & 0 & 0 \\
\hline $\mathrm{CB} / \mathrm{AO}$ & Constant & - & - \\
\hline $\mathrm{C}^{\prime} \mathrm{B}^{\prime} / \mathrm{A}^{\prime} \mathrm{O}$, & 0 & 0 & Free \\
\hline
\end{tabular}

\subsubsection{Application}

\subsubsection{Equivalent Shear Stiffness}

Fig. 4.9 plots shear stiffness vs. aspect ratio, where aspect ratio is defined as $h / a$. The lower bound was given by Davalos et al. (2001) and $\mathrm{Xu}$ et al. (2001) without considering skin effect. From Fig. 4.9, we can observe that, the analytical solution, considering shear warping, corresponds to hinge connection. There is a significant difference between hinge and rigid cases when the aspect ratio is low, whereas all the solutions approach the lower bound value as aspect ratio increases. This proves that, as pointed out by several researchers ( $\mathrm{Xu}$ et al. 2001), the skin effect is localized, and its effect on stiffness, which is a global parameter, is negligible when the core is high 
enough. However, the skin effect does affect the stresses significantly, as will be discussed next.

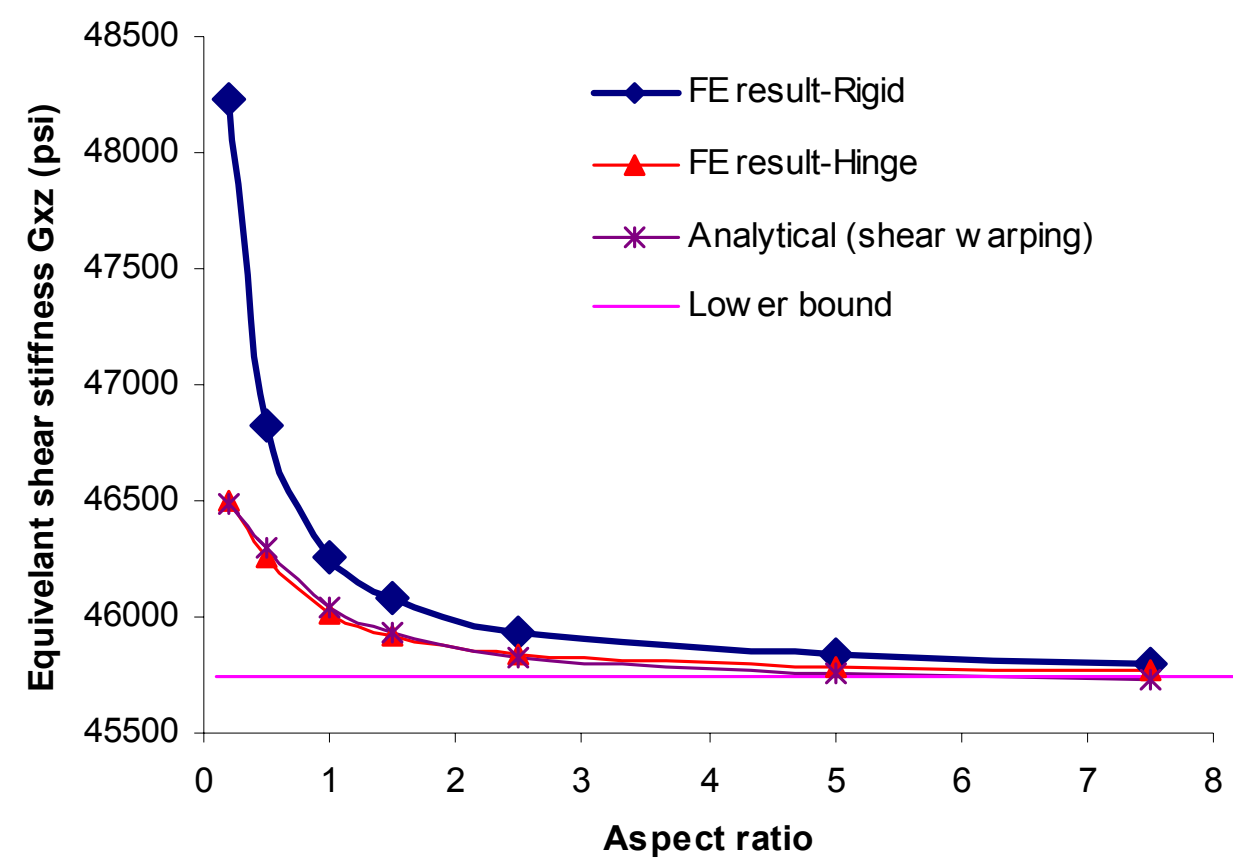

Figure 4.9 Stiffness vs. aspect ratio

Using regression technique, we can express the transverse shear stiffness as

$$
G_{x z}=45810+2477 e^{-\frac{R-0.1127}{0.2136}}+831 e^{-\frac{R-0.1127}{1.2393}}
$$

for rigid connection, where $\mathrm{R}$ is the aspect ratio, and

$$
G_{x z}=45709+747 e^{-\frac{R-0.1144}{0.6438}}+143 e^{-\frac{R-0.1144}{9}}
$$


for pinned connection from FE result, and

$$
G_{x z}=45524+758 e^{-\frac{R-0.1113}{0.7987}}+143 e^{-\frac{R-0.1113}{20}}
$$

for pinned connection from analytical result.

Therefore, the stiffness is a function of the aspect ratio $R$. Eq. (4.24) acts as a lower limit, and can be used in the analysis and design for safety reason.

It is also interesting to point out that, as concluded by Kelsey et al. (1958), the theory of minimum potential energy, a kinematically compatible uniform strain field, gives an upper bound; and the theory of complementary energy, a statically compatible uniform stress field, gives a lower bound, corresponding to infinitely large and zero skin effect, respectively. Voigt and Reuss (see Christensen, 1991) expressed this theory in parallel and series model as

$$
\begin{aligned}
& \frac{1}{2} \frac{\sigma_{i j}{ }^{2}}{C_{i j}} V \leq \sum_{k=1}^{n}\left(U_{b}+U_{s}+U_{a}\right)_{k} \\
& \frac{1}{2} C_{i j} \varepsilon_{i j}{ }^{2} V \leq \sum_{k=1}^{n}\left(U_{b}+U_{s}+U_{a}\right)_{k}
\end{aligned}
$$

where $k$ accounts for individual substructures in the RVE, and $U_{b}, U_{s}$, and $U_{a}$ are, respectively, the strain energies related to bending, shear, and axial responses. Eqs. (4.25) and (4.26) define, respectively, the conditions of lower and upper bounds for stiffness. Davalos et al. (2001) used these two equations to give an upper (47,580 psi) and lower 
bound $(45,750$ psi). Comparing these two values with the results shown in Fig. 4.9, we can note that the lower bound still applies, while the upper bound does not exist any more. This, once again, can be explained by the fact that bending warping was neglected in previous studies, and therefore, the stiffness was under-estimated.

\subsubsection{Stress Distribution}

Both analytical method and FE method are applied to a particular example; the core panel height is $\mathrm{h}=2$ ". The results are listed in Fig. 4.10 through Fig. 4.13.

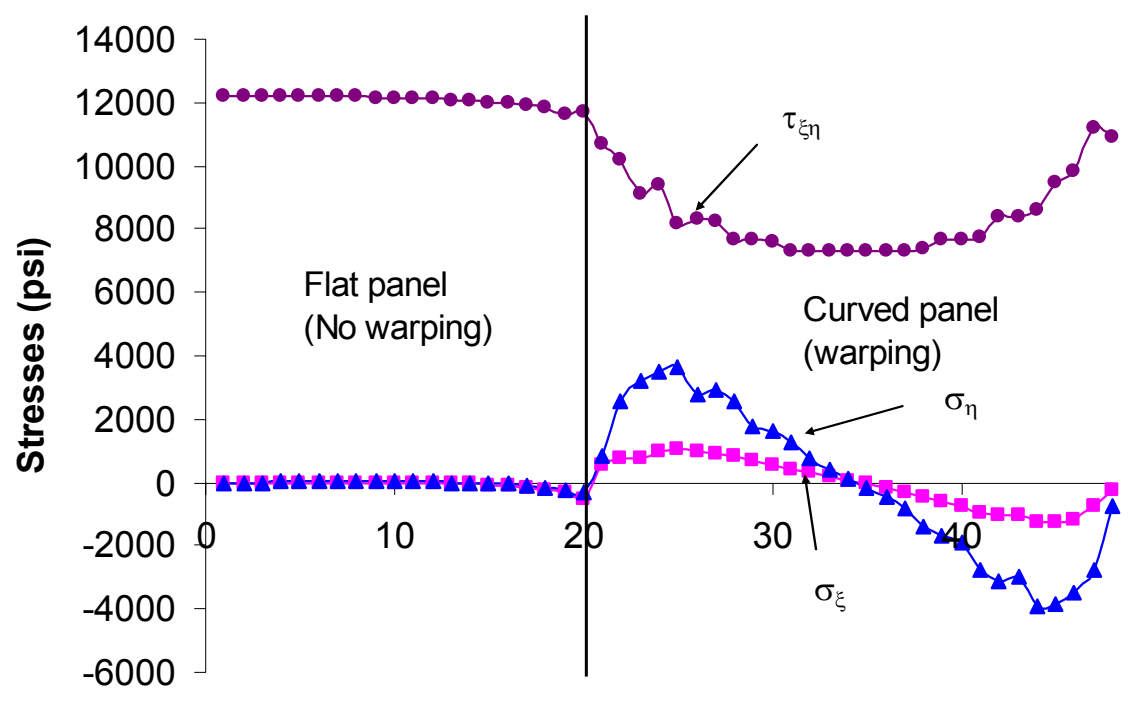

Element number

Figure 4.10 Stress distribution 


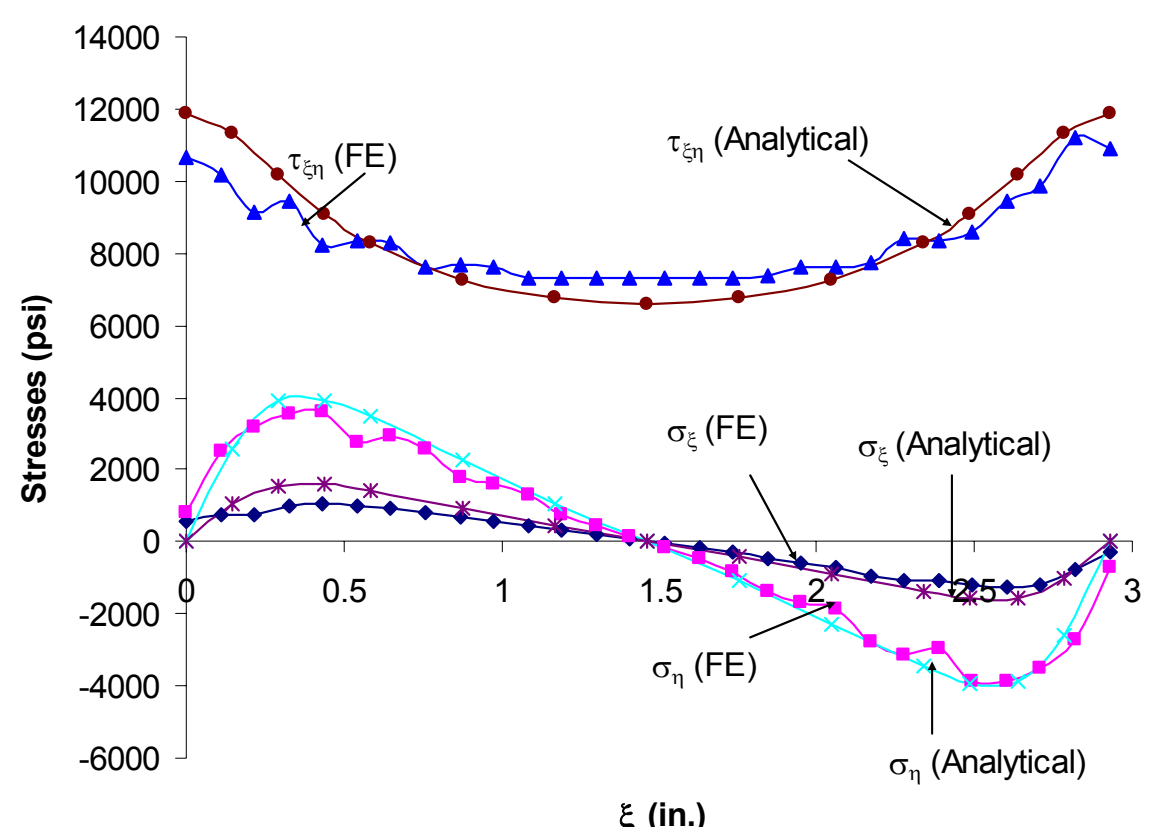

Figure 4.11 Comparisons between FE and analytical result for stress distribution (along length)

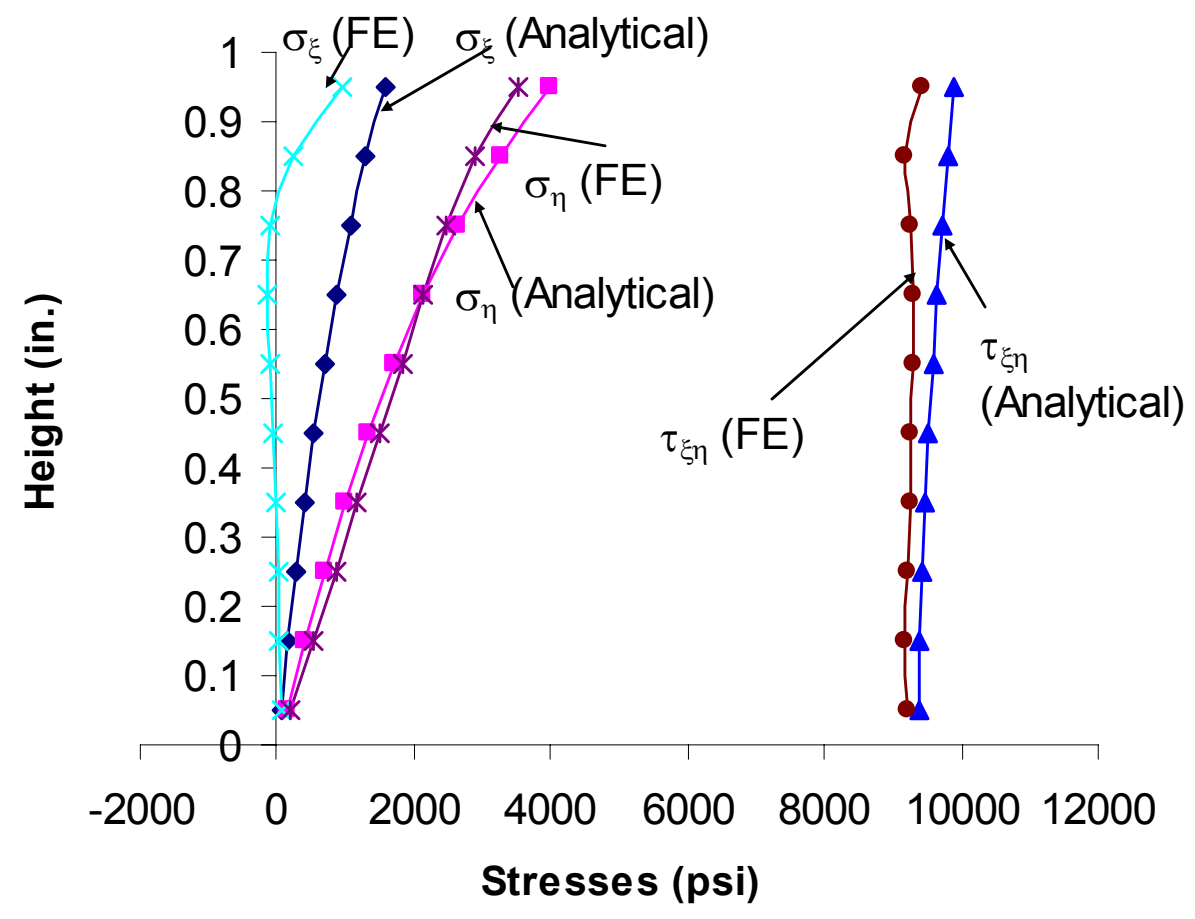

Figure 4.12 Comparisons between FE and analytical result for stress distribution (along height) 


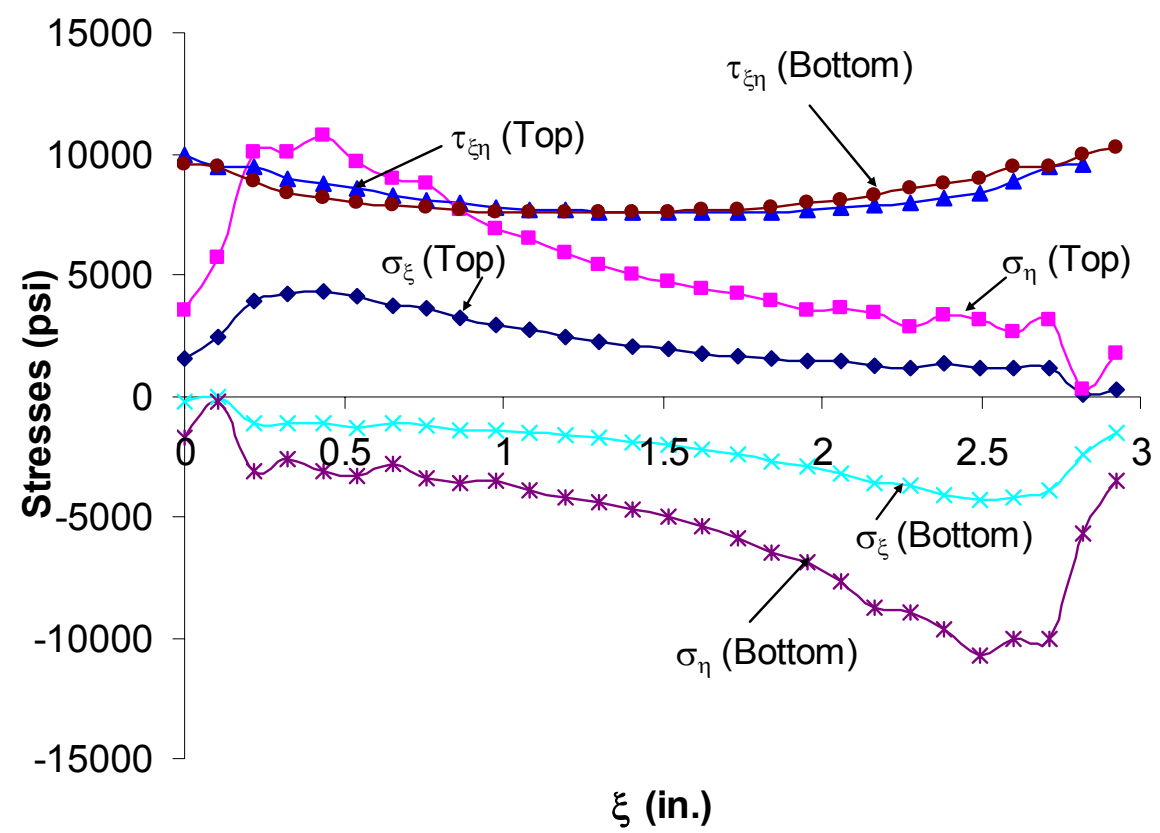

Figure 4.13 Stress distribution with bending warping based on the FE analysis

\section{Shear warping}

In order to illustrate the shear warping effect, all stresses are plotted in Fig. 4.10 for both flat and curved panels. From Fig. 4.10, we can note that, in the flat panel, the stress distribution is not affected, and the shear stress remains constant and the normal stress is essentially zero. While for the curved panel, the shear warping effect is significant; the minimum shear stress occurs at the center of the curved panel; and the distribution of normal stress is as shown in Fig. 4.10.

Fig. 4.11 plots the stress distributions along the top of the curved panel, as calculated both from analytical and FE results, showing good correlations. The same phenomenon can be observed for stress distribution along the height at the panel 
intersection, as shown in Fig. 4.12. This proves the accuracy of the analytical method for predicting the behavior of the curved panel under shear warping.

\section{Bending warping}

Fig. 4.13 shows the stress distributions for the curved panel assuming rigid connection between core and facesheet, from which we can note that, due to the bending effect explained above, the normal stress is no longer constant along the thickness $t$ of the core wall. Stresses, as positive at top and negative at bottom of the core wall section, result from the extra bending moment due to the rotation incompatibility. The shear stress distribution is also affected, the value of which decreases compared to the hinge connection, illustrating the benefits that rigid connection can offer.

\section{Discussion}

From Fig. 4.11, we observe that the ratio between the interfacial shear stress $(11,870 \mathrm{psi})$ and the interfacial tensile stress $(3,932)$ is approximately 3 . If bending warping is considered, the tensile stress can be even larger than the shear stress as shown in Fig. 4.13. Based on the results from the Flatwise Tension test and interfacial shear test, Wang (2004) pointed out that a typical interfacial shear strength $(1,750 \mathrm{psi})$ is $4-5$ times of the interfacial tensile strength (400 psi). Therefore, it is reasonably to assume that the delamination is caused by the tensile force at the interface (corresponding to Mode I facture). The tensile force can be used to predict the onset of the delamination. Once the crack occurs, there is a stress singularity at the crack tip, and facture mechanics method 
should be used to predict crack growth, using parameters such as facture toughness, crack length, J-Integral, etc.

\subsubsection{Parametric study}

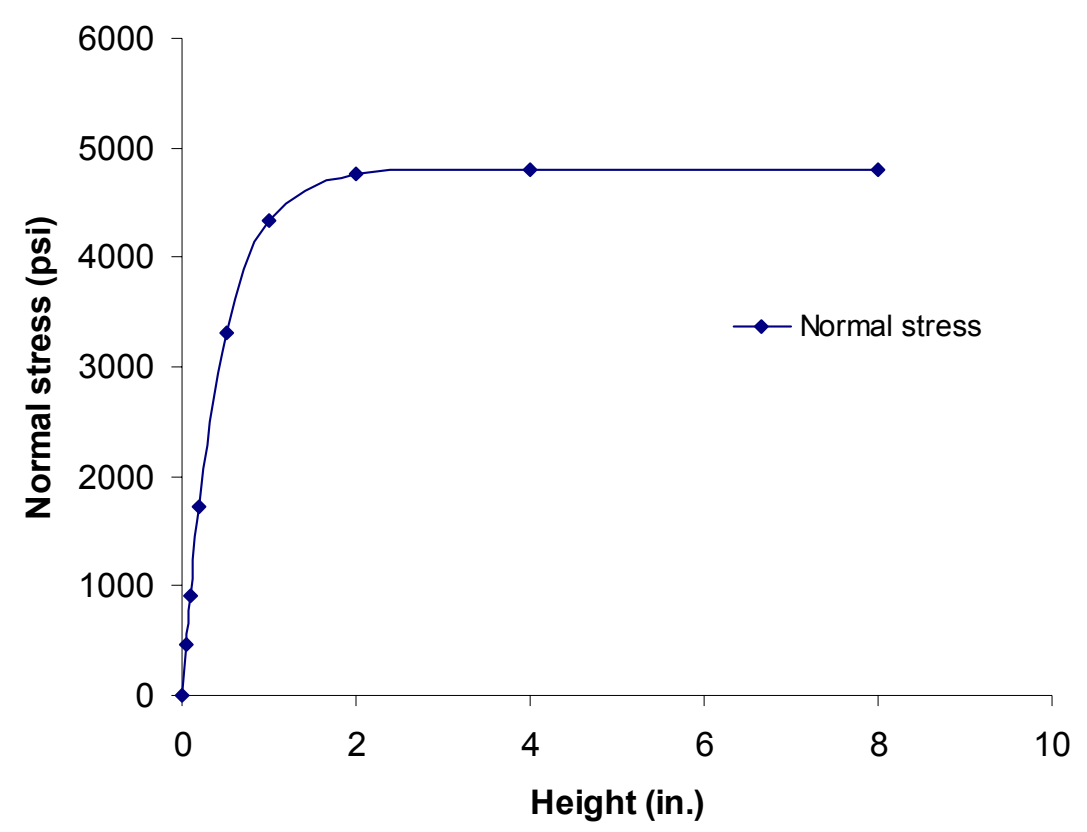

Figure 4.14 Normal interfacial stresses vs. height

Using the closed-form solution derived in this chapter, a parametric study is carried out for the interfacial normal stress, $S_{22}$, at the panel intersection under a shear strain of 0.02 , as shown in Fig. 4.14, from which we can observe that $S_{22}$ increases as the aspect ratio increases, and reaches a constant value beyond a certain limit, for instance, $h \approx 2^{\prime \prime}$ for this case. The curve shown in Fig 4.14 can be fitted using 


$$
S_{22}=4803-4803 e^{-(2.3172 h)^{1.0221}}
$$

Eq. (4.27) is based on a shear strain of 0.02. For a unit shear strain, Eq. (4.27) can be normalized as

$$
S_{22}=\gamma\left(240150-240150 e^{-(2.3172 h)^{1.0221}}\right)
$$

where $\gamma$ is the shear strain.

\subsubsection{Summary}

In this section, the skin effect, composed of shear and bending warping, on the behavior of HFRP sandwich sinusoidal core panels, is for the first time investigated. An analytical solution is given for shear warping and FE analyses are carried out for both shear and bending warping cases. It is concluded that:

1. The analytical solution can successfully predict the behavior of curved panels considering shear warping, which is verified by FE results.

2. Skin effect includes two parts: shear and bending warping. Shear warping corresponds to cases with hinge connection between facesheet and core, and when both warping effects are considered, it corresponds to a rigid connection. Actual cases lie between these two conditions.

3. The skin effect is a localized phenomenon. The lower bound of the equivalent stiffness can thereby be adopted if the aspect ratio is high enough. However, it can 
significantly affect interface stress distribution, yielding a coupled stress state for the curved panel, where the normal stress may even be larger than the shear stress. This indicates, unlike the common belief that only shear stress occurs when the structure is under pure shear force, that tensile force at the interface arises, making it a potentially critical component. Therefore, special considerations are suggested for design purposes.

4. The skin effects described herein only affect the stress distribution of the curved panel and has no effect on the flat panel. This effect on the stress distribution becomes less significant in the area away from the interface.

5. Practical formulas to calculate equivalent shear stiffness and interfacial normal stress are provided. Together with flatwise tension test results, they can be used for failure predictions, as will be shown in Section 4.7. 


\subsection{CER Effect on Shear Stiffness and Interfacial Shear Stress Distribution}

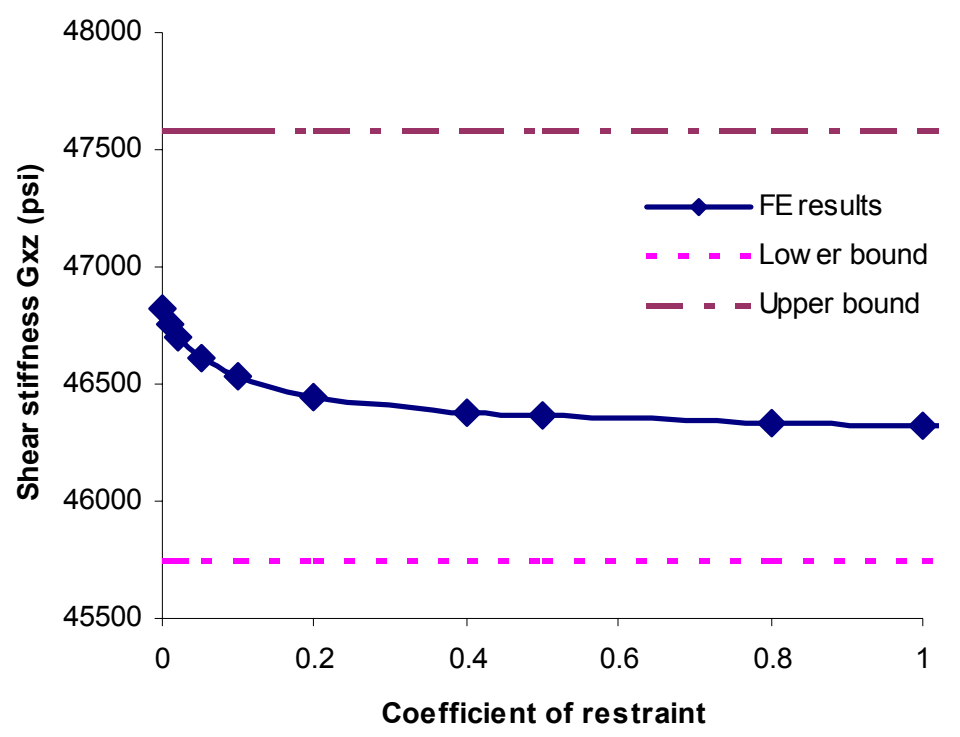

Figure 4.15 CER effect on transverse shear stiffness

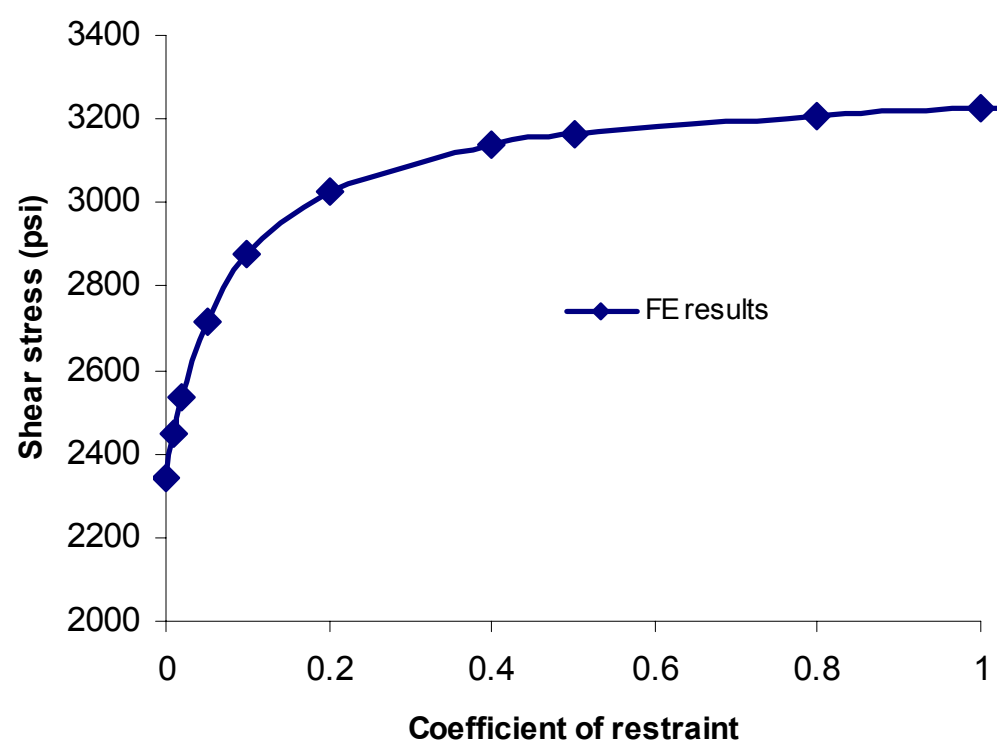

Figure 4.16 CER effect on interfacial shear stress 
In Chapter 3, we concluded that CER effect will greatly affect the buckling strength of core panels under out-of-plane compression. It is interesting to find out that this effect can also contribute to the shear stiffness and interfacial shear distribution. The same model shown in Section 4.2.5 is employed, and spring element, as described in Chapter 3 , is put at the interface to simulate the partially constrained condition.

Fig. 4.15 plots the FE results for $G_{x z}$ vs. CER curve, from which we can note that completely rigid boundary conditions $(\mathrm{CER}=0)$ correspond to the largest value of $G_{x z}$. The FE results fall within the range of the lower (45,750 psi) and upper bound (47,580 psi) solutions given by Davalos et al. (2001). However, the absolute maximum difference is $1.2 \%$, which is negligible.

From FE analysis, the shear stress contour indicates that the shear stress at top nodes is uniform except in the area adjacent to the connection of the flat and sinusoidal wave panel, where the shear stress decreases. Therefore, this nearly uniform stress can represent the interfacial shear stress. Fig. 4.16 displays the relationship between CER and interface shear stress, from which we can see that shear stress increases as CER increases, with maximum values for near hinged conditions $(\mathrm{CER} \geq 1.0)$. Therefore, the shear stress corresponding to hinged condition can be adopted to predict shear crushing failure for design purposes. 


\subsection{Shear buckling}

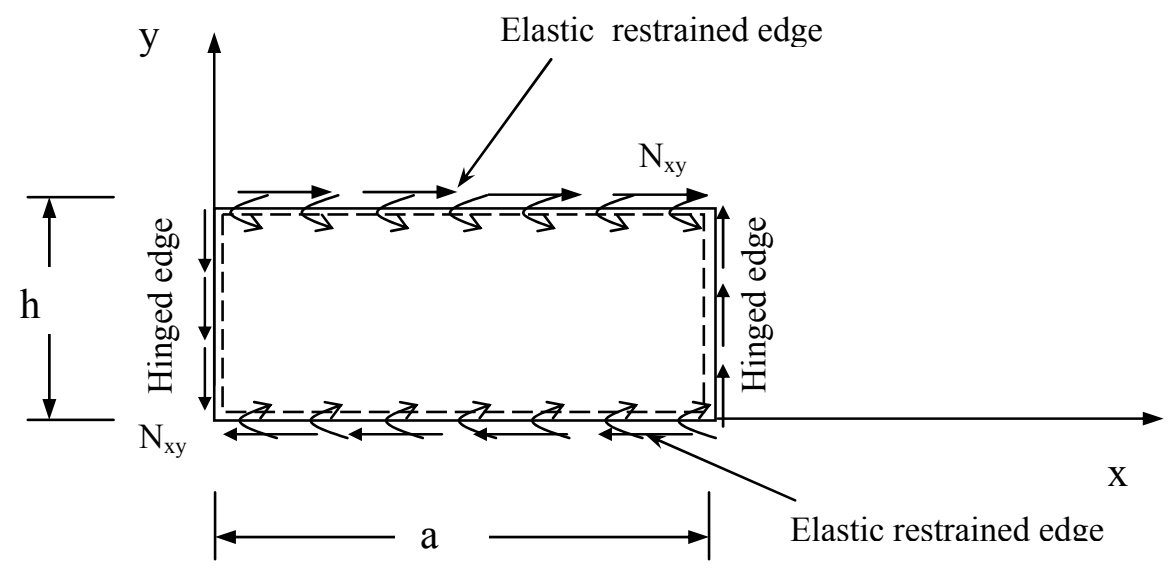

Figure 4.17 Boundary condition of FRP plate

The core may buckle due to shear loading if the core is deep and thin. The solution for shear buckling is provided. Following the approach given by Qiao et al. (2001) and considering the boundary conditions shown in Fig. 4.17, the following first variation of the total potential energy equation is used to define the problem,

$$
\begin{aligned}
& \int_{0}^{a} \int_{0}^{h}\left(D_{11} \frac{\partial^{2} w}{\partial x^{2}} \delta \frac{\partial^{2} w}{\partial x^{2}}+D_{12} \frac{\partial^{2} w}{\partial x^{2}} \delta \frac{\partial^{2} w}{\partial y^{2}}+D_{12} \delta \frac{\partial^{2} w}{\partial x^{2}} \frac{\partial^{2} w}{\partial y^{2}}\right. \\
& +D_{12} \frac{\partial^{2} w}{\partial x^{2}} \delta \frac{\partial^{2} w}{\partial y^{2}}+D_{22} \frac{\partial^{2} w}{\partial y^{2}} \delta \frac{\partial^{2} w}{\partial y^{2}}+4 D_{66} \frac{\partial^{2} w}{\partial x \partial y} \delta \frac{\partial^{2} w}{\partial x \partial y} \\
& \left.+N_{x y} \frac{\partial w}{\partial x} \delta \frac{\partial w}{\partial y}+N_{x y} \delta \frac{\partial w}{\partial x} \frac{\partial w}{\partial y}\right) d x d y \\
& +\int_{0}^{a}\left[\bar{\zeta}\left(\frac{\partial w}{\partial y}\right)_{y=0} \delta\left(\frac{\partial w}{\partial y}\right)_{y=0}+\bar{\zeta}\left(\frac{\partial w}{\partial y}\right)_{y=h} \delta\left(\frac{\partial w}{\partial y}\right)_{y=h}\right] d x=0
\end{aligned}
$$


Using the Rayleigh-Ritz method, the displacement $w(x, y)$ that satisfies the boundary conditions (excluding the case when the boundary conditions are clamped, i.e., $\bar{\zeta}=\infty$ ) can be defined as

$$
w=\sum_{i=1}^{m} \sum_{j=1}^{n} A_{i j} \sin \frac{i \pi x}{a} \sin \frac{j \pi y}{h}
$$

Substituting Eq. (4.30) into Eq. (4.29), a typical eigenvalue problem results. The results of the eigenvalues are in the form of pairs of \pm quantities, which means there is no direction requirement for the shear stress. The smallest eigenvalue can be taken as the critical shear stress resultant. Fig. 4.18 shows the critical shear stress of shear buckling for one bonding layer and two core thickness. An asymptotic value can be assumed for the aspect ratio $h / a>5$, when a sufficient number of terms (e.g., $\mathrm{m}=\mathrm{n}=6$ ) is included (Qiao et al., 2001). The critical buckling stress for different bonding layers are shown in Fig. 4.19, from which we can observe that the difference for the bonding layers effect on shear buckling capacity is negligible. 


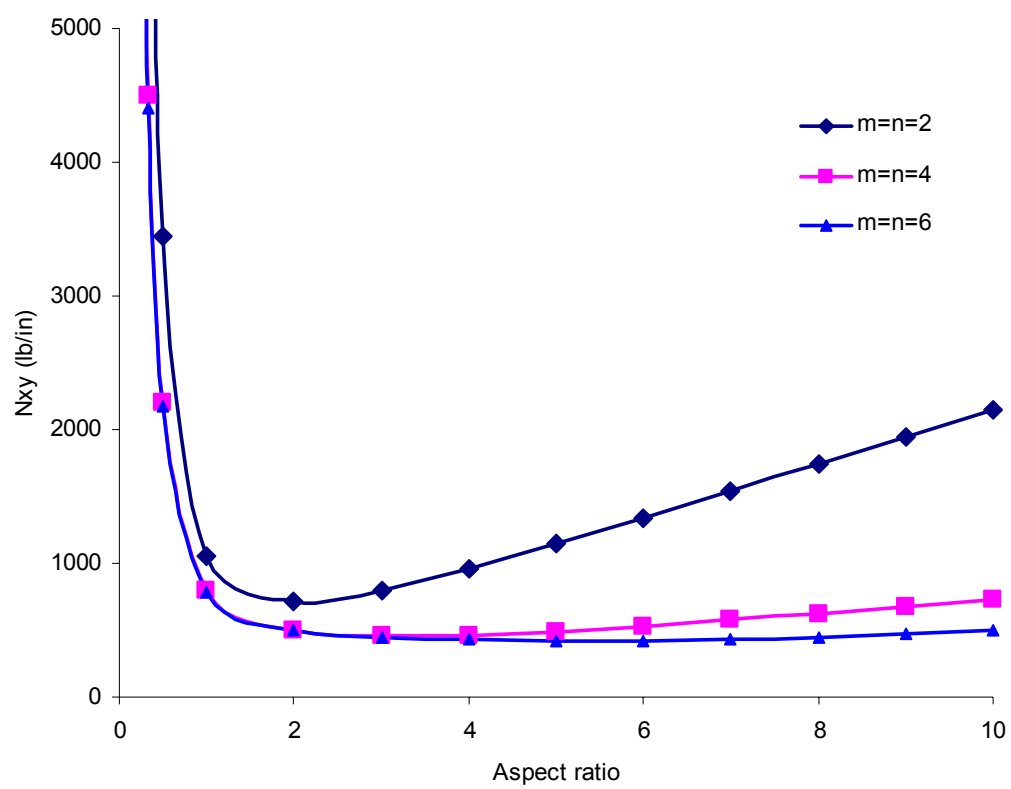

Figure 4.18 Critical shear stress vs. aspect ratio for one bonding layer

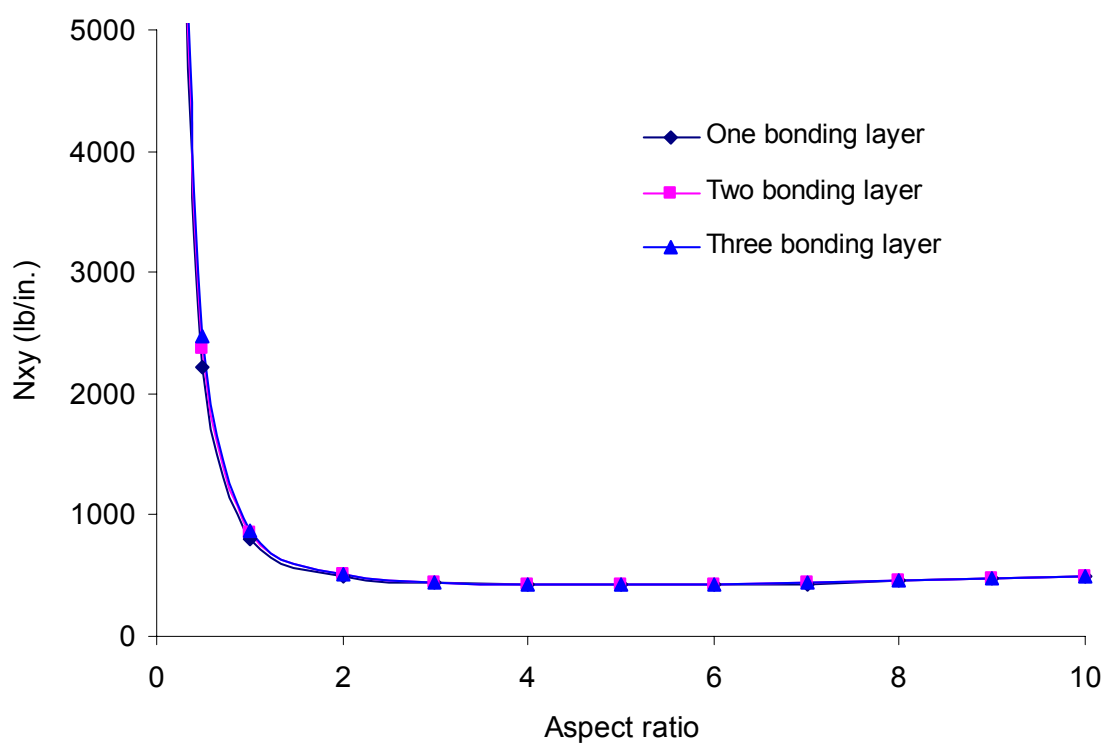

Figure 4.19 Critical shear stress vs. aspect ratio for different bonding layers 
The curves shown in Fig. 4.19 can be fitted using

$$
N=A_{1} e^{-\frac{R}{t_{1}}}+A_{2} e^{-\frac{R}{t_{2}}}+N_{0}
$$

where $N$ is the critical shear stress and $R$ is the aspect ratio. The parameters corresponding to different bonding layers are listed in Table 4.3. The shear stress can be expressed as

$$
\tau=\frac{N}{t}=\frac{A_{1}}{t} e^{-\frac{R}{t_{1}}}+\frac{A_{2}}{t} e^{-\frac{R}{t_{2}}}+\frac{N_{0}}{t}
$$

where $t$ is the core wall thickness.

Table 4.3 Parameters for design equation

\begin{tabular}{|c|c|c|c|c|c|}
\hline & $\mathrm{A}_{1}$ & $\mathrm{t}_{1}$ & $\mathrm{~A}_{2}$ & $\mathrm{t}_{2}$ & $\mathrm{~N}_{0}$ \\
\hline One bonding layer & 2103 & 0.5326 & 34611 & 0.1388 & 448 \\
\hline Two bonding layers & 2661 & 0.5097 & 37093 & 0.1355 & 449 \\
\hline Three bonding layers & 3015 & 0.4970 & 38734 & 0.1339 & 450 \\
\hline
\end{tabular}




\subsection{Proposed Method to Predict Failure Load}

It is shown (Caprino and Langella, 2000) that if the core Young's modulus is negligible with respect to the facing elastic modulus, and the facing thickness is small compared to the height of the core, the transverse shear stress field in the core is practically uniform. Therefore, the following basic assumptions are adopted in this model:

1) Transverse shear stress is carried by the core only;

2) Transverse shear stress is uniformly distributed along the core height;

3) The structure is considered to fail once the transverse shear stress exceeds the critical shear strength, either shear strength of the material or buckling strength.

\subsubsection{Core-face Delamination}

From discussion above, we can observe that when under pure shear force, tensile force at the interface arises, making it a potentially critical component. Therefore, special considerations are suggested for design purposes. Based on the analytical model derived in this section, we can propose the following design guidelines using the failure criterion of maximum stress:

1) For a given loading condition, calculate shear strain based on the equivalent shear modulus by Eq. (4.24);

2) Find the interfacial tensile stress from Eq. (4.28) using shear strain calculated from Step 1; 
3) Compare this interfacial tensile stress with the interfacial tensile strength from flatwise tension test.

This method will provide a conservative result since 1) The shear stiffness corresponding to the hinged connection between core and facesheet is adopted; and 2) it is shown (Wang, 2004) that a crack is initiated when the interface traction attain the interfacial strength, and the crack is advanced when the work of traction equal to the material's resistance to crack propagation. Therefore, a nominal interfacial tensile strength will be used in order to propose a more reasonable criterion. The validity of the proposed method will be discussed through the correlation with four-point bending test results as will be shown in Section 4.7.

\subsubsection{Core Shear Failure and Shear Buckling}

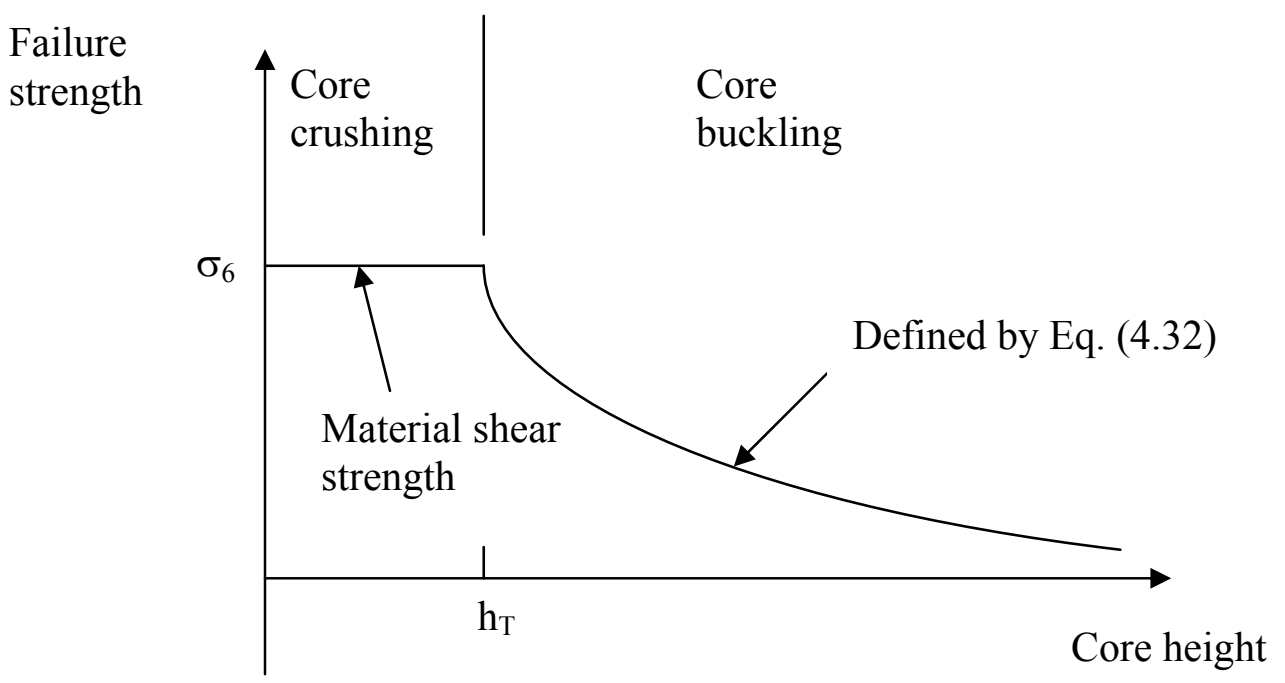

Figure 4.20 Design diagram 
Table 4.4 Transition height

\begin{tabular}{|c|c|c|c|}
\hline & One bonding layer & Two bonding layer & Three bonding layer \\
\hline $\mathrm{h}_{\mathrm{T}}$ & $3.48 ”$ & $3.72{ }^{\prime}$, & $3.84 ”$ \\
\hline
\end{tabular}

From the analysis shown above, it is found that the shear stress in the flat panel is higher than that in the curved panel. Therefore, the flat panel is more critical when considering pure shear failure and shear buckling. Following the same method for compressive strength, we can propose a design equation as shown in Fig. 4.20, where $\mathrm{h}_{\mathrm{T}}$ is the height where the failure mode transits from core crushing to core buckling, as listed in Table 4.4.

The material shear strength can be obtained from V-notched test (Iosipescu test, ASTM D5379-98), as shown in Appendix B. The average value of five specimens give $\sigma_{6}=10,239$ psi.

Following the same approach described in Section 3.8, the following design guidelines are proposed for the shear capacity of flat panel:

1) For a given loading condition, calculate shear strain based on the equivalent shear modulus by Eq. (4.24);

2) Calculate shear stress in the flat panel;

3) Compare the shear stress with the strength obtained from Fig. 4.20.

This method will be illustrated in Section 4.7. 


\subsection{Experimental Investigation}

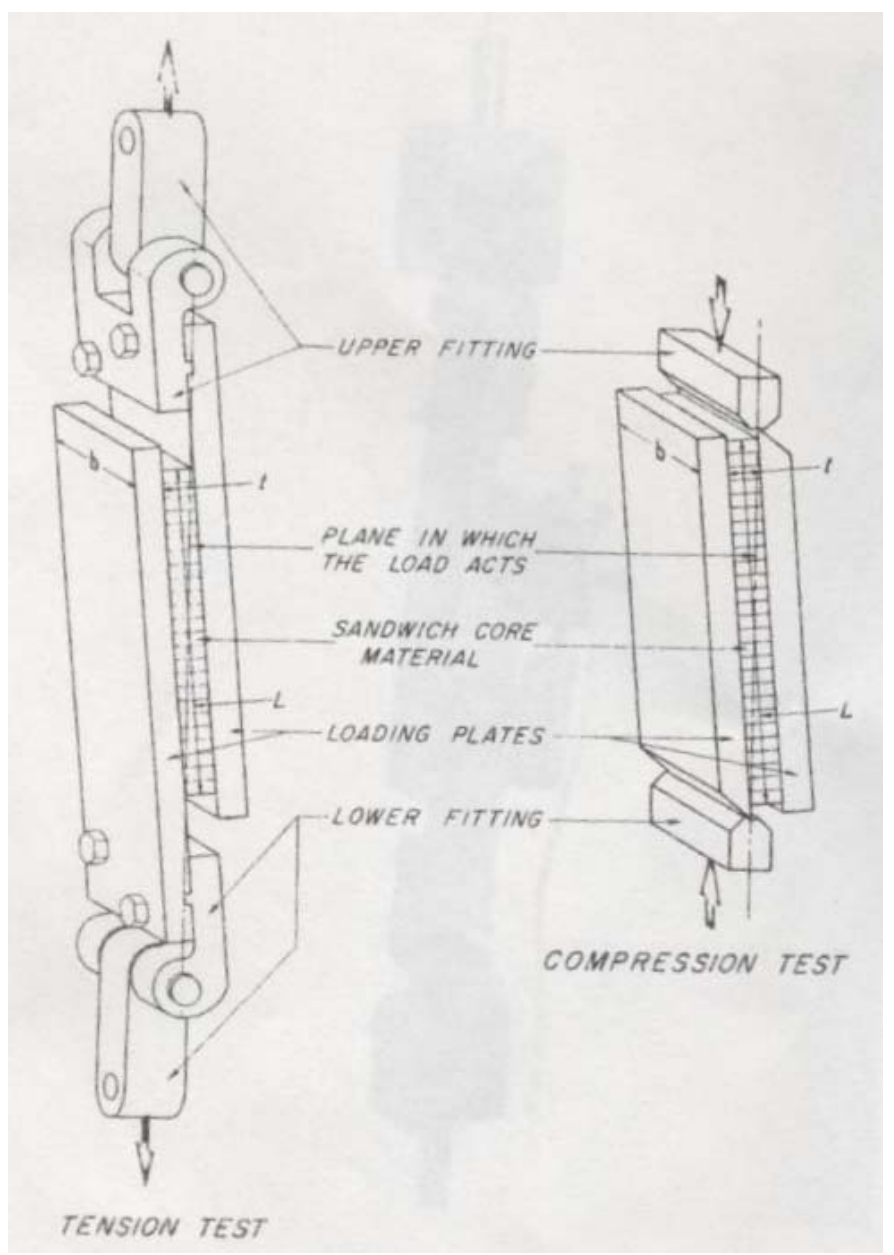

Figure 4.21 Plate shear specimens

It is recommended that ASTM C273-00 be used for shear properties of sandwich core materials, as shown in Fig. 4.21, which was also initially adopted in this study. It was finally abandoned because it was found from trial tests that, due to high shear strength of the core material, the delamination in the facesheet, i.e., intra-laminate delamination, occurred well before the shear failure of the core material can be achieved. Fortunately, another method, four-point bending test (ASTM C393-00), is also recommended by ASTM for the study of core shear strength and shear modulus, which 
was used by a lot of researchers as shown in the literature review, and is also adopted herein.

\subsubsection{Test Description}
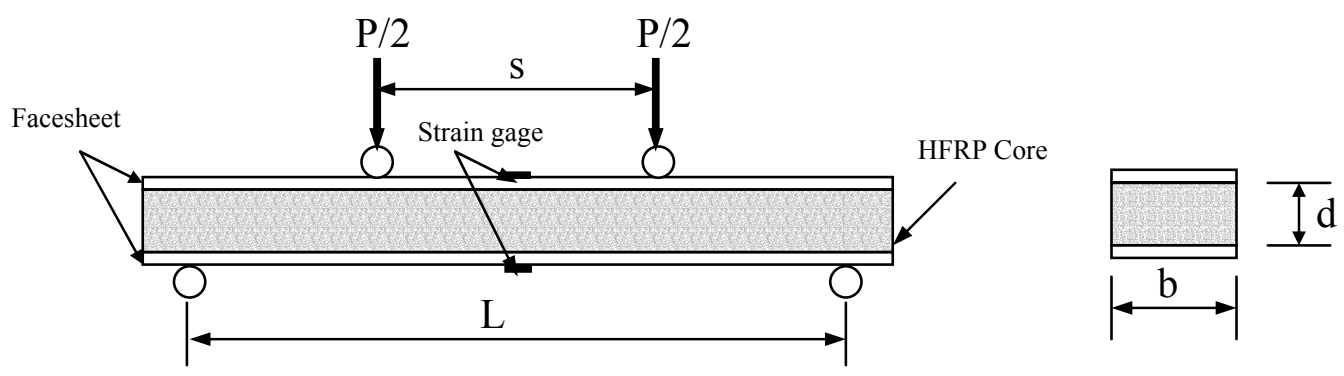

Figure 4.22 Test setup

The dimensions of the specimen were 28 " long by 4 " wide by 2 " deep. There were seven single cells either along the longitudinal or transverse direction, as shown in Fig. 4.22. To minimize the influence of the layers of the facesheet other than the bonding layer on the strength of the specimen, only a combined $0 \%$ ContSM layer is placed over the ChSM bonding layer, as shown in Fig. 3.6. The constituent materials of the facesheet are given in Fig. 3.6, and their properties are provided in Table 3.4, with the properties of each component material given in Table 3.5.

The core of the sandwich panels was "embedded" into the facesheet using a ChSM contact layer and resin. The number of these bonding layers was varied from one to three to study their effect on strength. Two types of beam samples were manufactured by orienting the sinusoidal wave: (1) along the length ("longitudinal"), and (2) along the width ("transverse"). All tests were carried out in accordance to ASTM standards. Fig. 4.22 displays the test setup, where $\mathrm{L}=24$ " and $\mathrm{s}=12$ ”. An external load cell was placed 
between the loading block and the specimen to record the load, and LVDTs were used to record the displacements. Two strain gages on the top and two on the bottom facesheets were bonded at the mid-span of the beam (Fig. 4.22). The test was performed at a displacement rate of $0.06 \% / \mathrm{min}$. A photo of test set up is shown in Fig. 4.23.

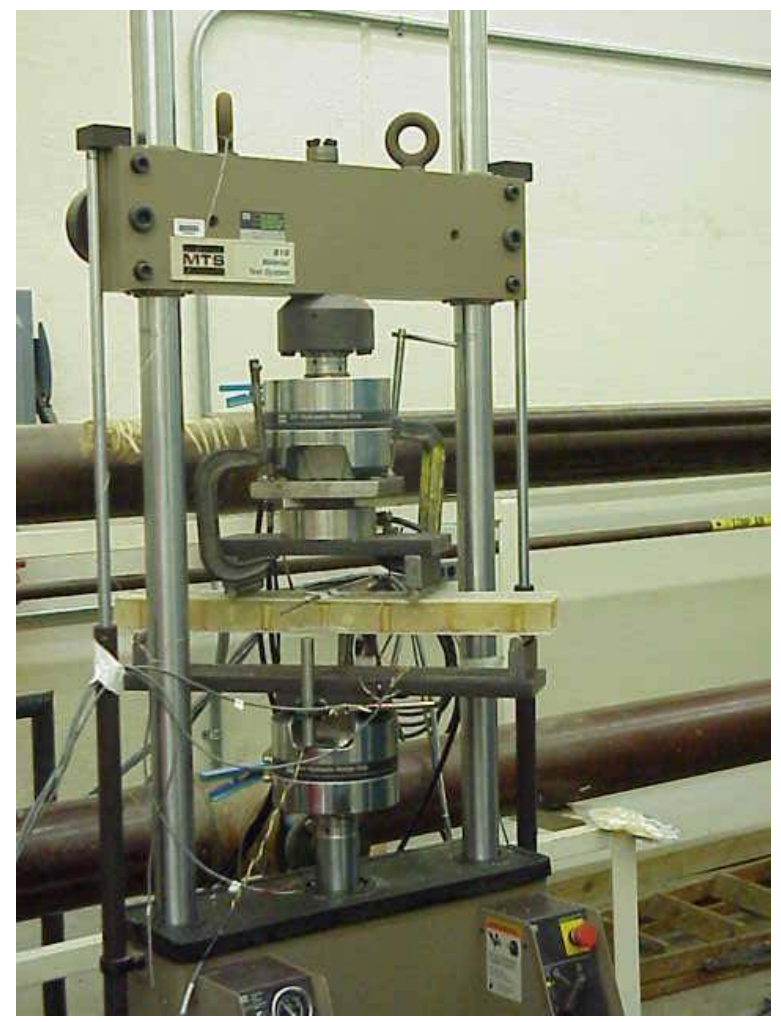

Figure 4.23 Photo of test setup 


\subsubsection{Test Results and Discussion}

\subsubsection{Longitudinal Test}

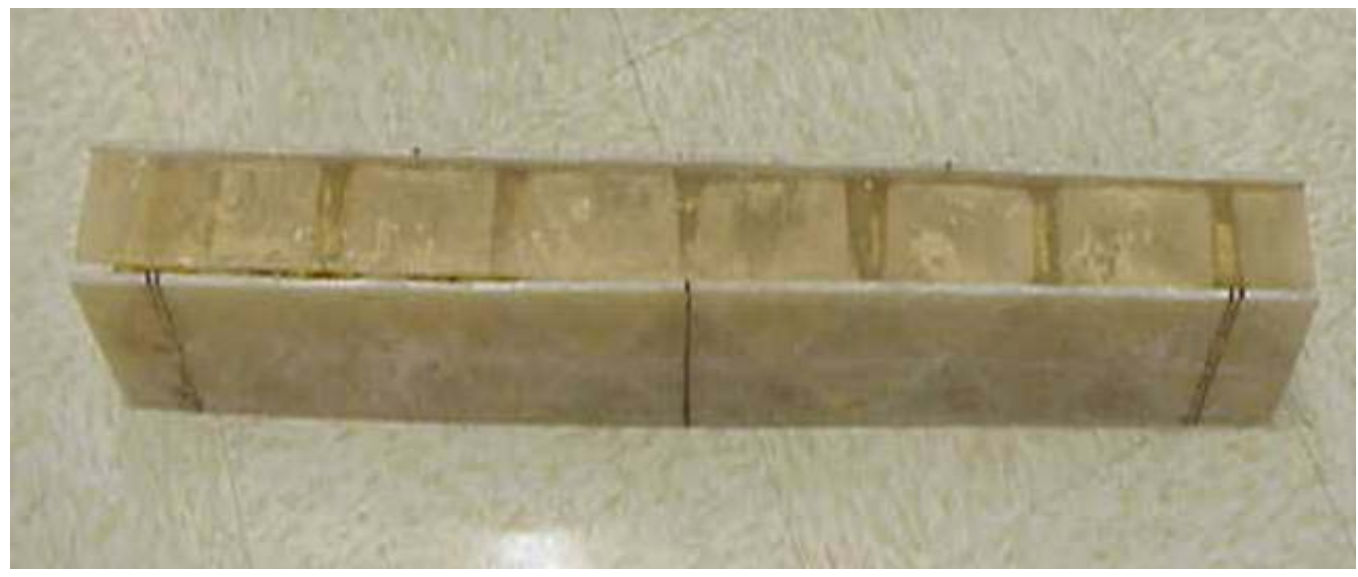

Figure 4.24 Failure due to delamination

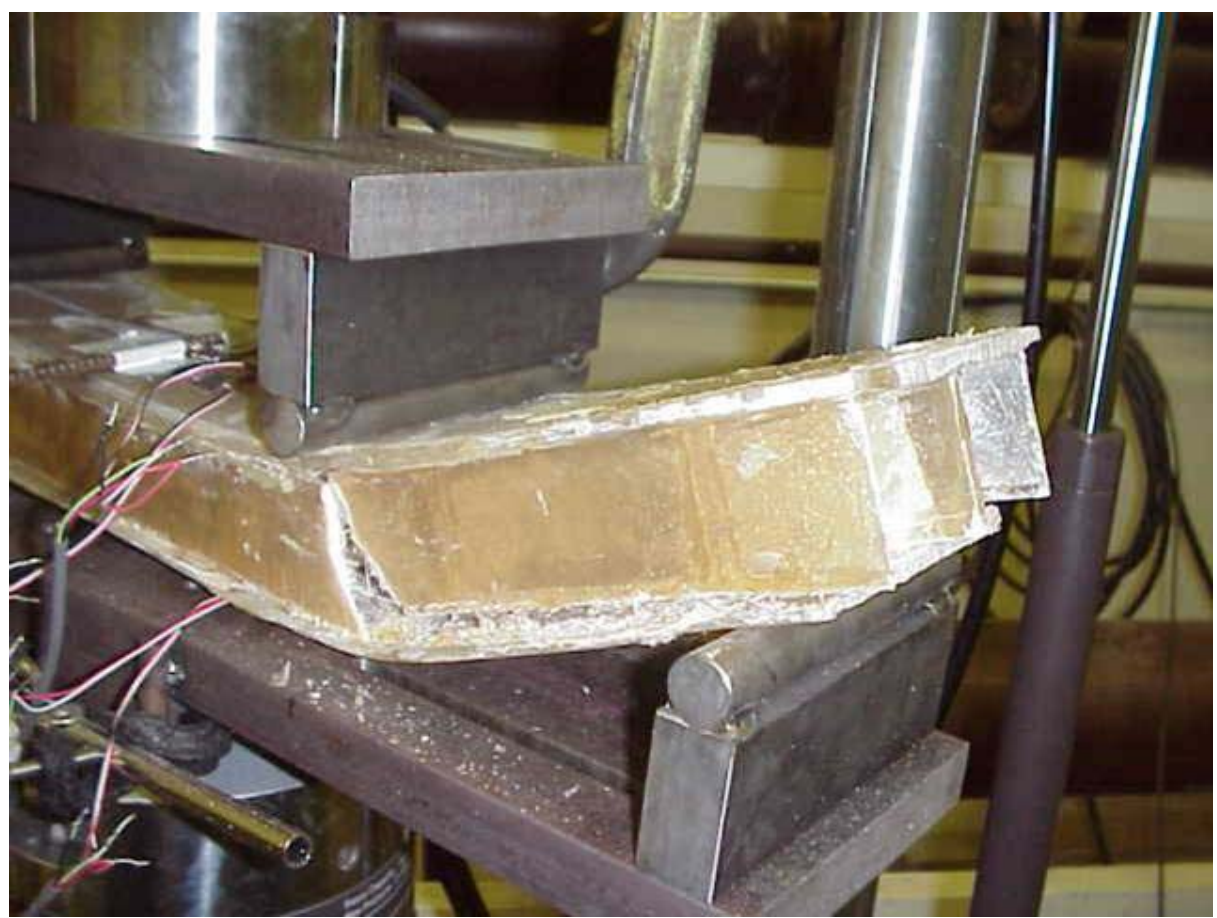

Figure 4.25 Shear crushing of the core 
Table 4.5 Average value of failure load for longitudinal samples

\begin{tabular}{|c|c|c|c|c|c|c|c|c|}
\hline & B1C2 & B2C2 & B3C2 & B2C1 & B2C3 & B3C1 & B3C3 & $\begin{array}{c}\text { Excessive } \\
\text { bonding } \\
\text { layers }\end{array}$ \\
\hline Average Value (1b) & 3750 & 5435 & 6780 & 3965 & 5285 & 5310 & 9310 & 15,840 \\
\hline Standard deviation (lb) & 200 & 490 & 840 & 375 & 800 & 410 & 740 & 3,985 \\
\hline
\end{tabular}

The beams under static loadings showed nearly linear-elastic behavior up to failure. The number of bonding layers affects the mechanical behavior of the specimens. For the one to three bonding layers, the failure of the specimens was due to a sudden debonding between the facesheet and the core material, as shown in Fig. 4.24. The energy stored in the specimen was released in a relatively short time resulting in a loud failure. For the excessive bonding layers, the facesheet did not delaminate from the core, and a typical shear failure of the core under the loading point occurred instead, as shown in Fig. 4.25 .

The average value of the maximum load of three specimens for excessive bonding layers and five specimens for each other type is given in Table 4.5, which shows that the magnitudes of failure loads are in the same relation as the number of bonding layers and core thickness; i.e., the specimen with three bonding layers is much stronger than that with one bonding layer, and specimen with three core thickness is much stronger than that with one core thickness, clearly showing that the effect of the number of bonding layers and core thickness plays an important role on the failure load. This is due to the fact that, by increasing the number of the bonding layer and the core thickness, larger fillets of excess adhesive are formed at honeycomb interface, and hence, increases the bonding area. Fig. 4.26a and Fig. 4.26b show the displacement at mid-span versus load 
curves for two bonding layers and two core thickness specimens. Fig. 4.27a and Fig. 4.27b show the load-strain curves for two bonding layers and two core thickness specimens. From these figures we can conclude that specimen exhibited an approximate linear behavior up to failure.

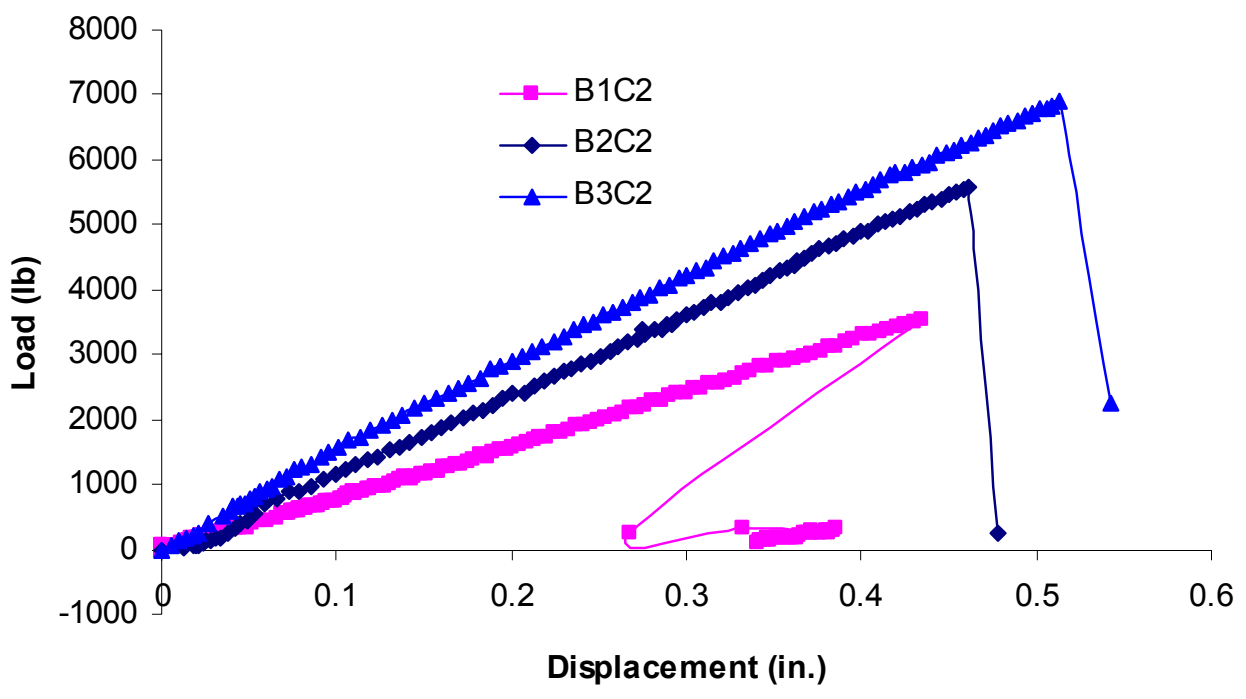

(a) Two core thickness

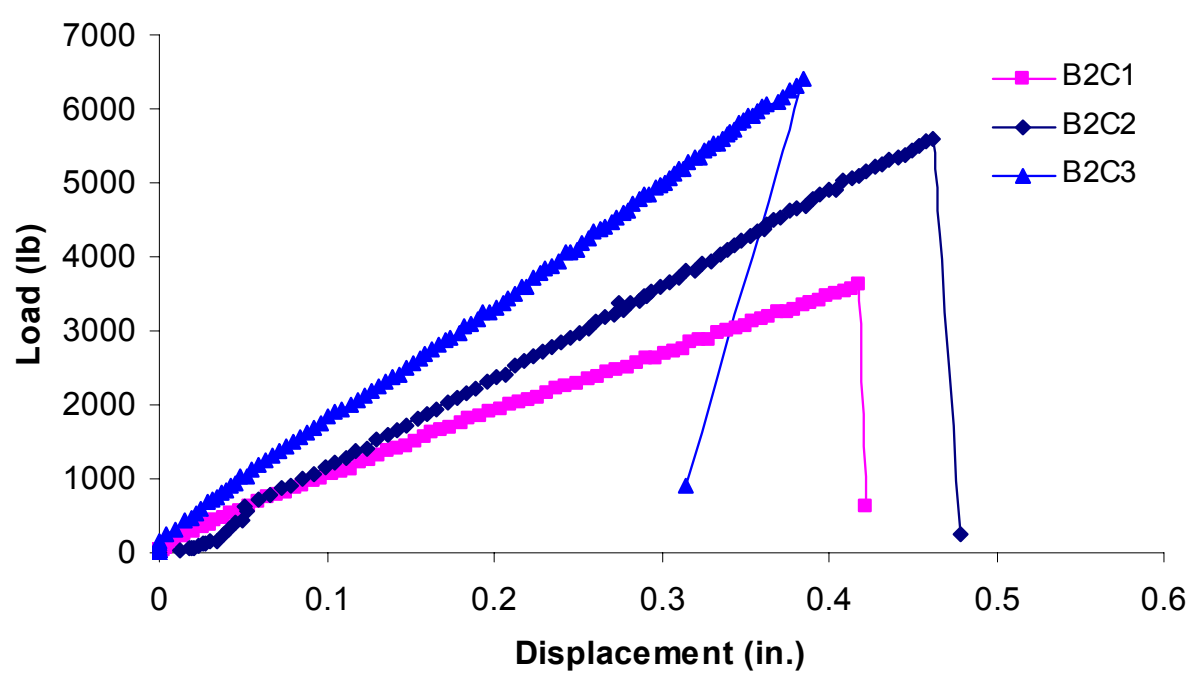

(b) Two bonding layers

Figure 4.26 Load-displacement curve for longitudinal test 


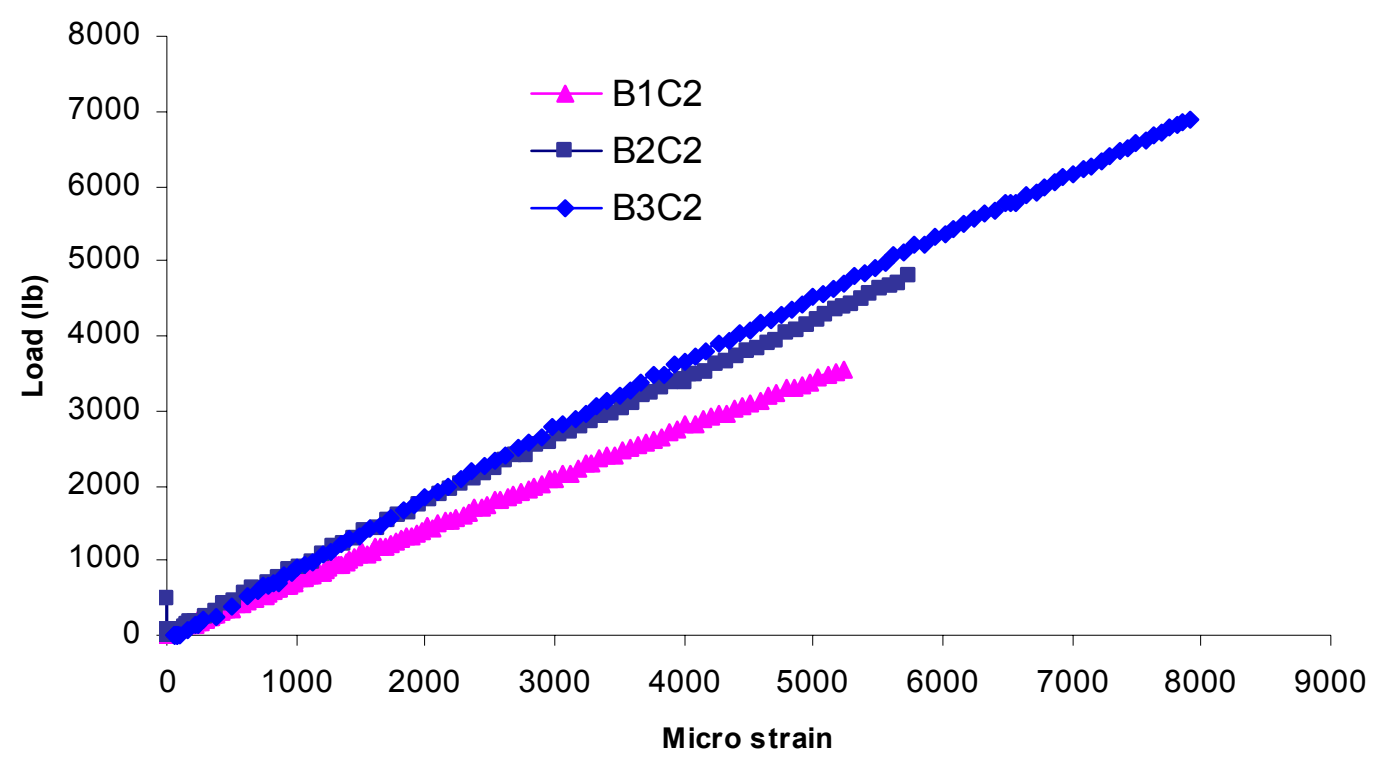

(a) Two core thickness

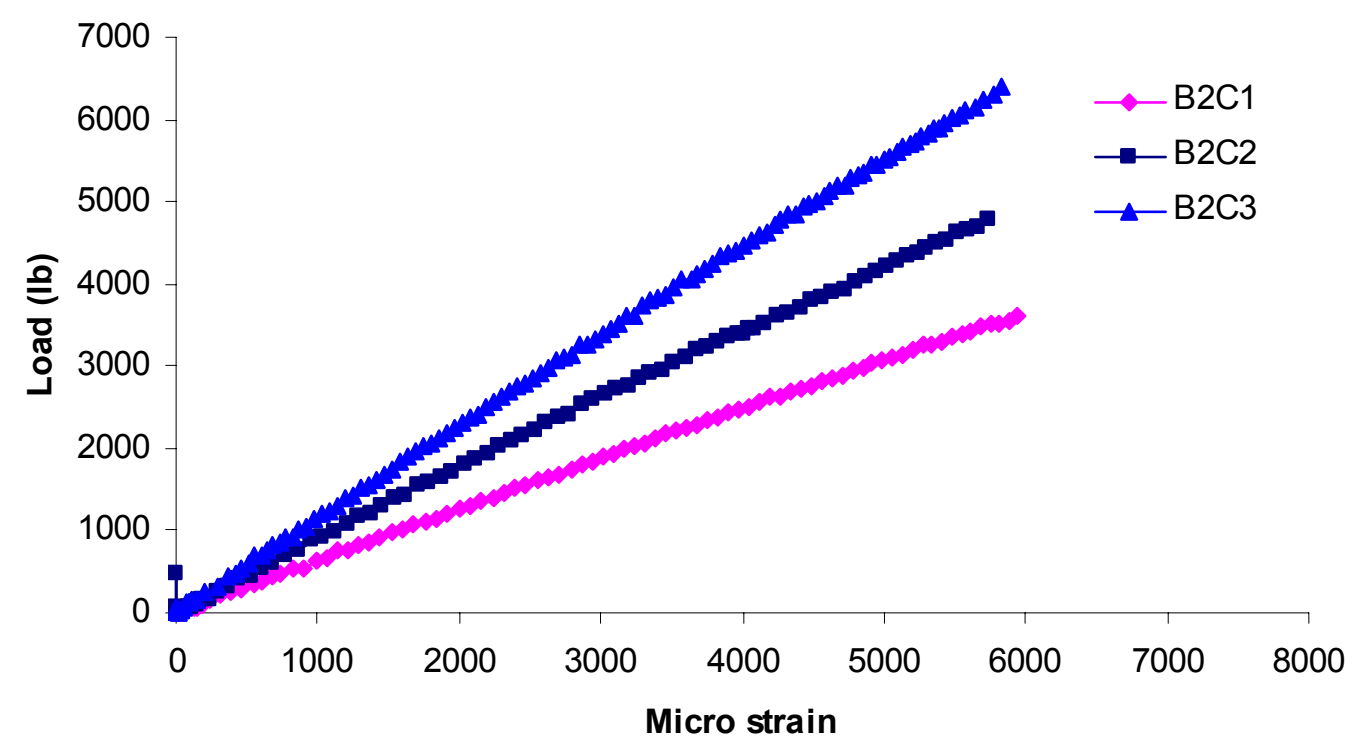

(b) Two bonding layers

Figure 4.27 Load-strain curve for longitudinal test 


\subsubsection{Transverse Test}

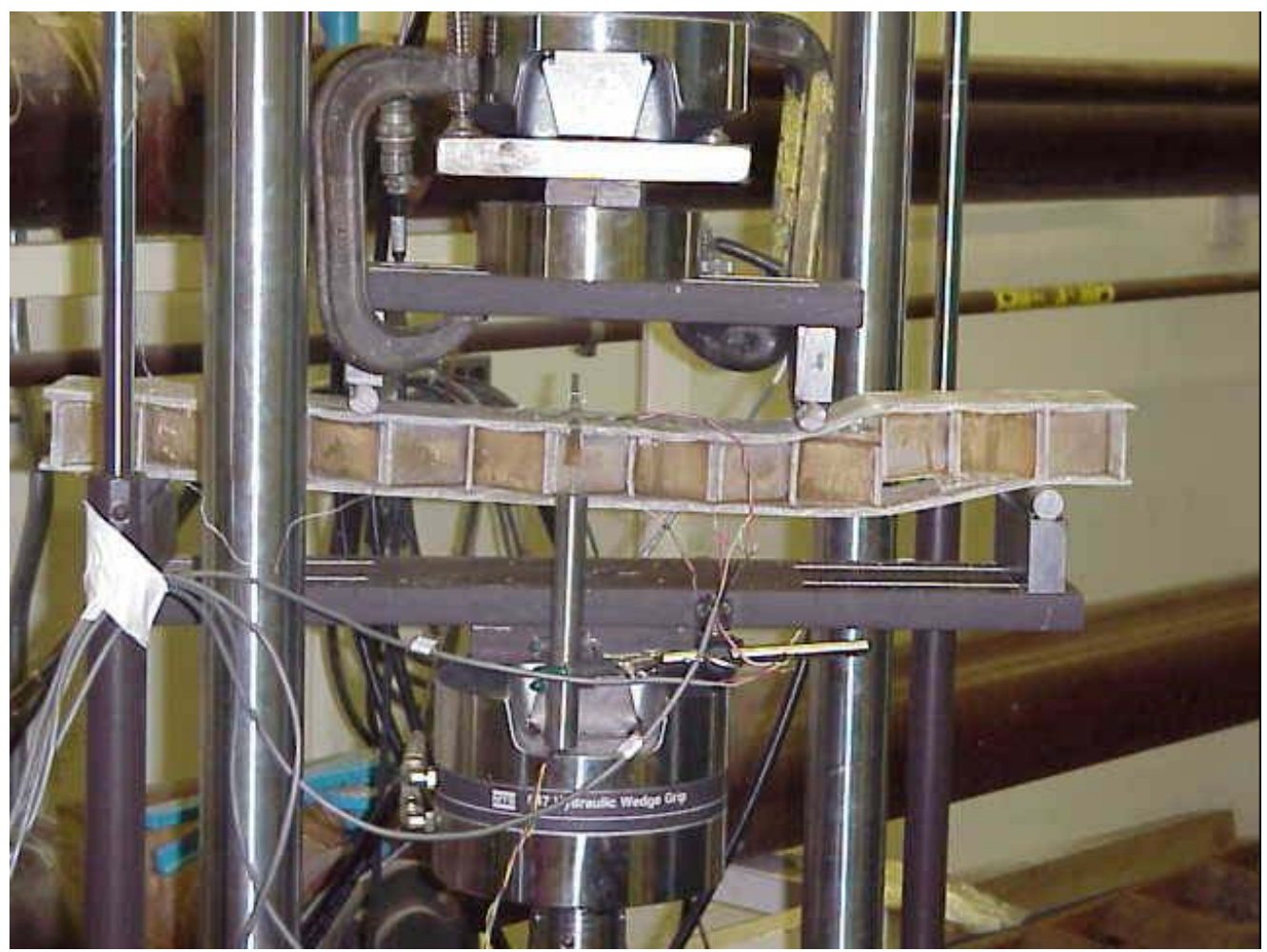

Figure 4.28 Core separation

All types of specimens displayed the same failure mode. The failure in the core was initiated by debonding at the contact area between the sinusoidal panel and flat panel, as shown in Fig. 4.28. The specimens continued to carry some load until the delamination between the facesheet and core material occurred. Unlike longitudinal specimens, the failure was not as sudden, and several rises and drops of load were observed during the test. 


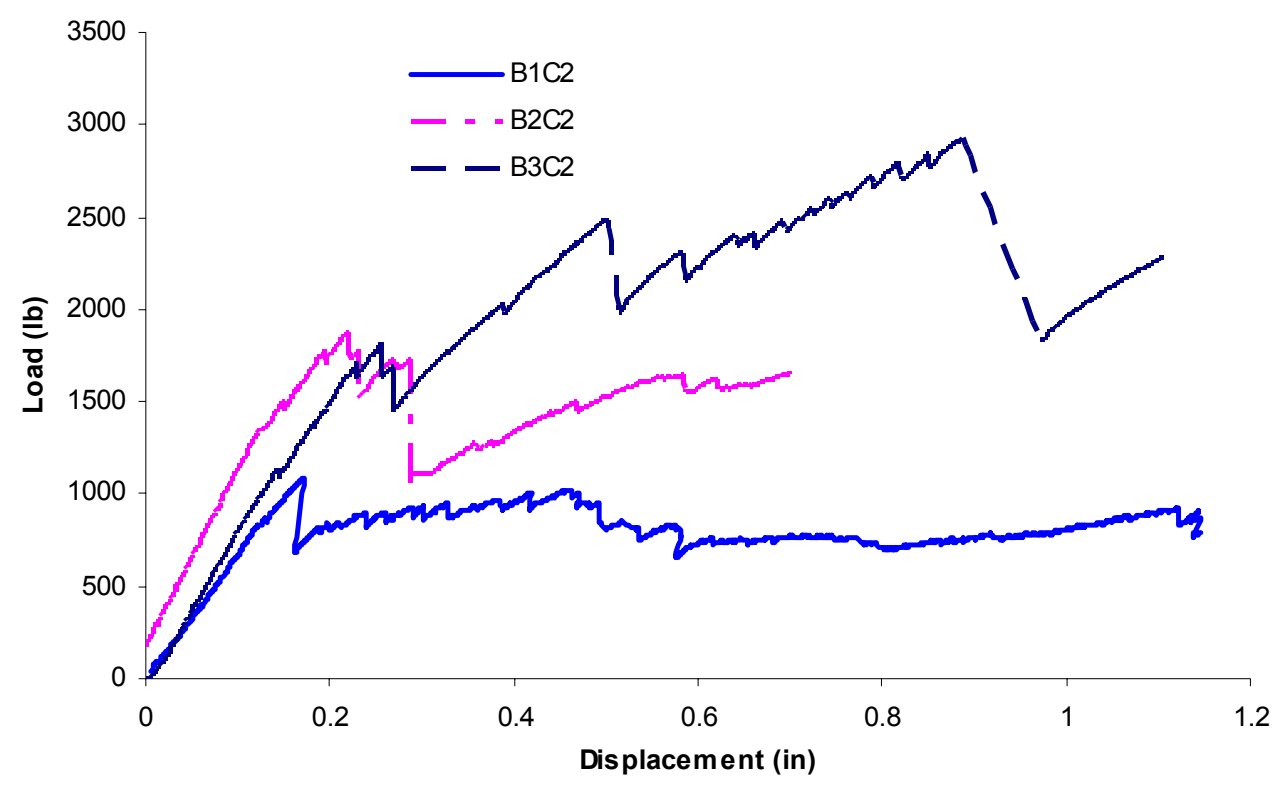

(a) Two core thickness

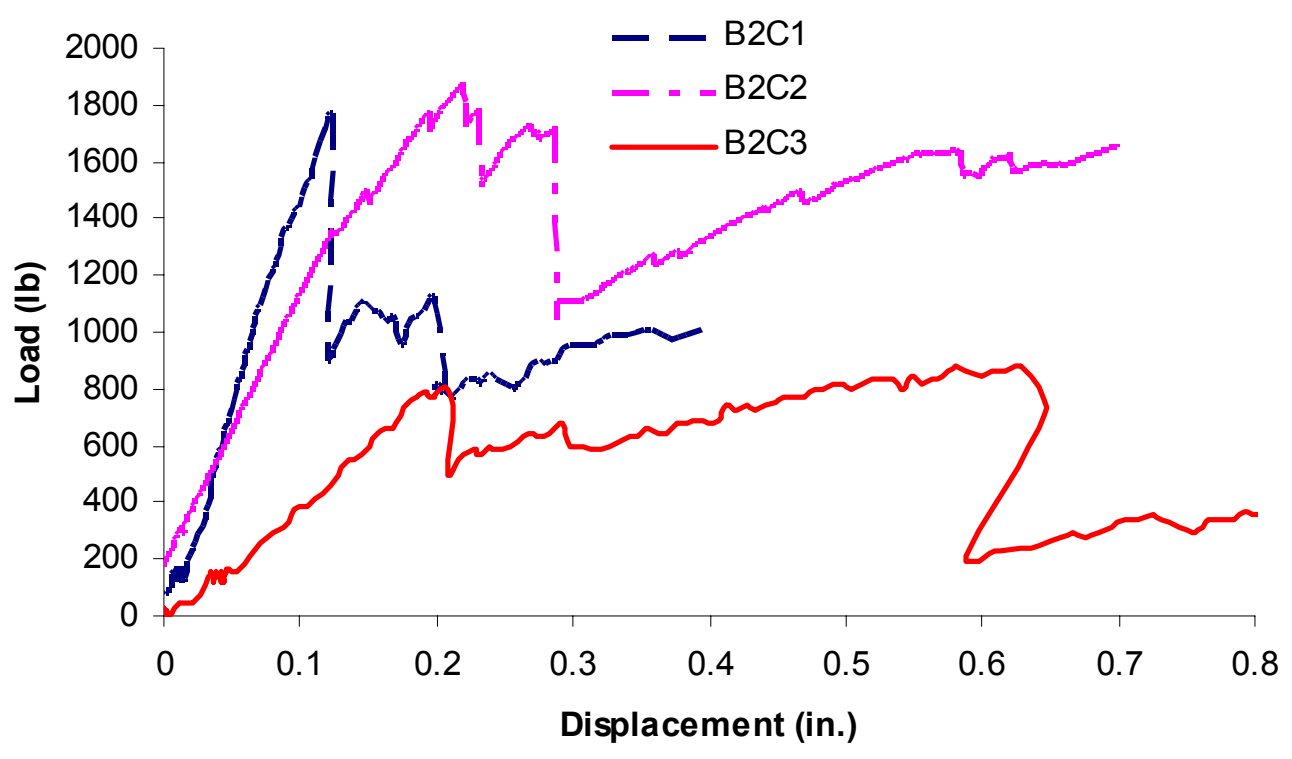

(b) Two bonding layers

Figure 4.29 Load-displacement curve for transverse test 


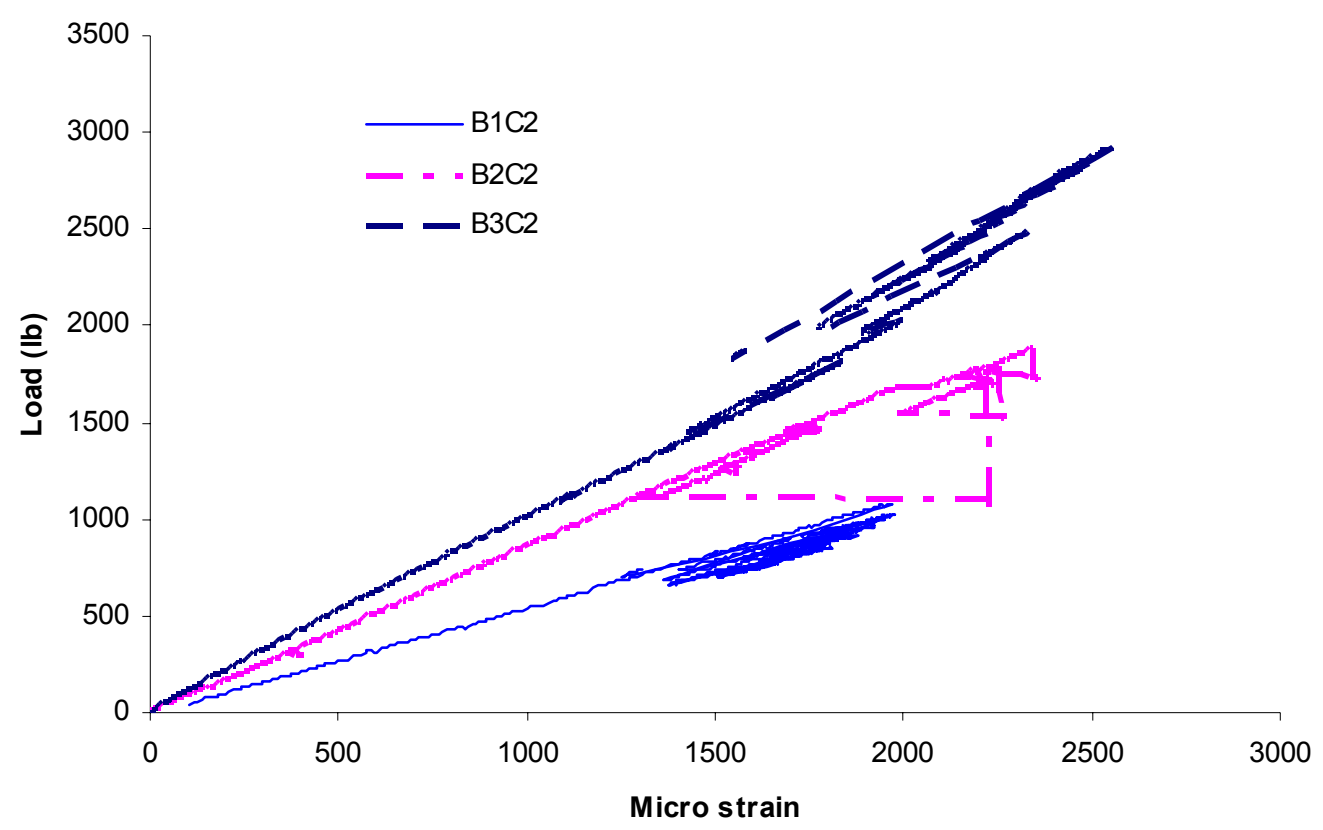

(a) Two core thickness

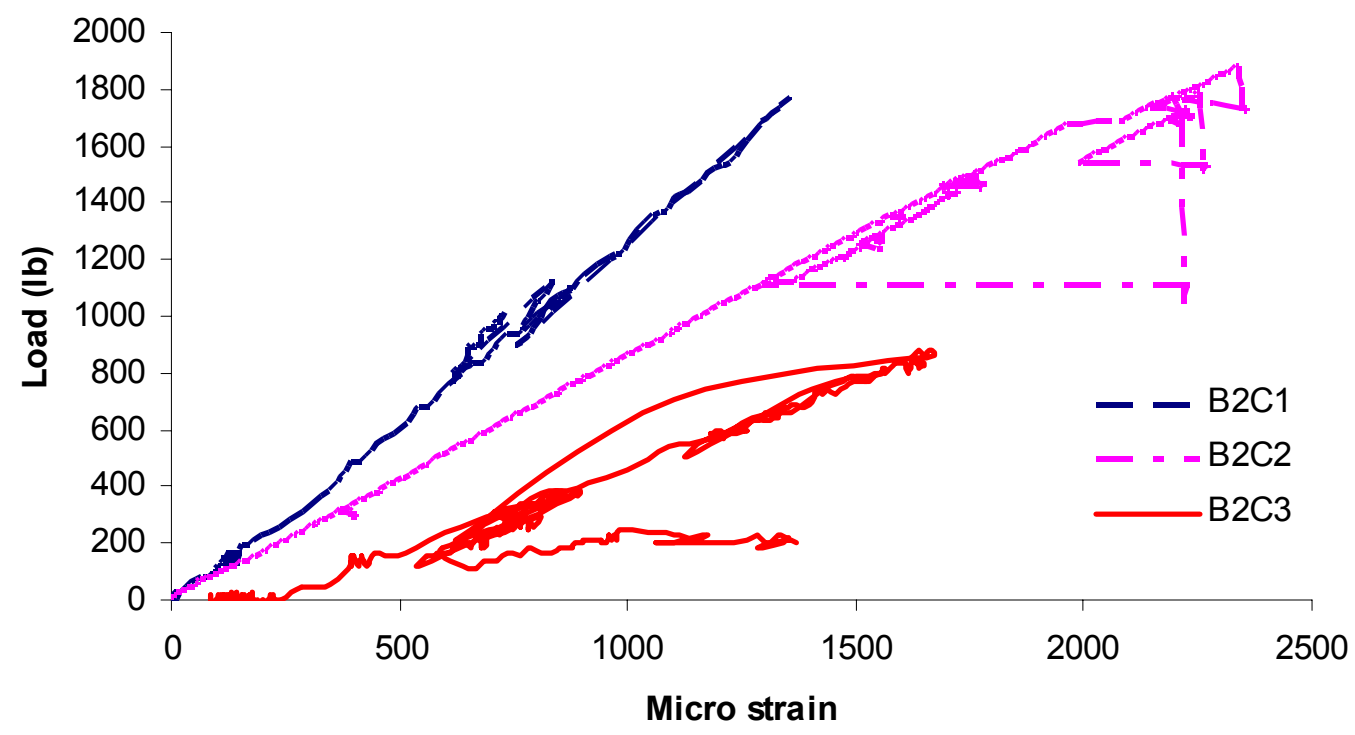

(b) Two bonding layers

Figure 4.30 Load-strain curve for transverse test 
Table 4.6 Average value of failure load for transverse samples

\begin{tabular}{|c|c|c|c|c|c|c|c|}
\hline & B1C2 & B2C2 & B3C2 & B2C1 & B2C3 & B3C1 & B3C3 \\
\hline Average Value (lb) & 1180 & 1570 & 2515 & 1190 & 1610 & 1525 & 2480 \\
\hline Standard deviation (lb) & 160 & 285 & 600 & 75 & 170 & 195 & 410 \\
\hline
\end{tabular}

The failure loads for five specimens each are given in Table 4.6, which shows much lower values compared with what we obtained for the longitudinal samples. Therefore, the transverse specimens should not be used when high shear stresses are expected. Fig. 4.29a and Fig. 4.29b show typical load-displacement curves for two bonding layers and two core thickness. Fig. 4.30a and Fig. 4.30b show typical load-strain curves for two bonding layer and two core thickness specimens. We can observe that the specimens follow a nearly linear behavior until first failure occurred.

\subsubsection{Summary}

An investigation on the strength properties of HFRP specimen in bending is conducted through four-point bending tests. In particular, the influence of facesheet-core interface bonding effect is examined by varying the bonding layers of the specimen. Two cases of bending tests are carried out: longitudinal and transverse bending test. It is found that:

1. All specimens followed an approximate linear behavior prior to failure in bending. The failure load for the longitudinal specimens is much higher than that for the transverse specimens. For longitudinal samples, the excessive bonding 
layers specimens failed in shear, and the other specimen types failed by debonding. All of the transverse specimens failed by debonding. Transverse-type beams should be avoided when high shear stresses are expected.

2. The failure load is sensitive to the bonding layer effect and core thickness effect. Specimens with more bonding layers and core thickness failed at a higher load than those with less bonding layer and core thickness. The failure load may vary for the same type of specimen due to the variability of bonding quality, which indicates the importance of quality control during manufacturing of the panels.

From the test result, we can observe, as expected, that the longitudinal samples are much stronger in shear than the transverse samples. The number of bonding layers and core thickness correspond clearly to the maximum strengths achieved. However, there is variability in results even for specimens with the same number of bonding layers, especially for the type with excessive bonding layer. One of the factors that may contribute to this variability is the bonding quality. For some specimens, the fillets are not well formed at the core-facesheet interface, resulting in minor cracks. This indicates the importance of quality control during the manufacturing process. 


\subsection{Correlations between Test Results and Prediction from Design Equations}

For longitudinal specimens, two types of failure modes were observed, pure shear failure and delamination. In this section, the models derived in Section 4.5 are used to predict the failure strength corresponding to these two distinct failure modes.

\subsubsection{Shear failure of Flat Panel}

Table 4.7 Parameters for sandwich beam specimen with excessive bonding layers

\begin{tabular}{|c|c|c|c|c|}
\hline $\mathrm{E}_{\mathrm{f}}\left(\mathrm{x} 10^{6} \mathrm{psi}\right)$ & $\mathrm{E}_{\mathrm{c}}\left(\mathrm{x} 10^{6} \mathrm{psi}\right)$ & $\mathrm{b}(\mathrm{in})$ & $\mathrm{d}(\mathrm{in})$ & $\mathrm{t}_{\mathrm{f}}(\mathrm{in})$ \\
\hline 1.94 & 0.077 & 4.5 & 2 & 0.2842 \\
\hline
\end{tabular}

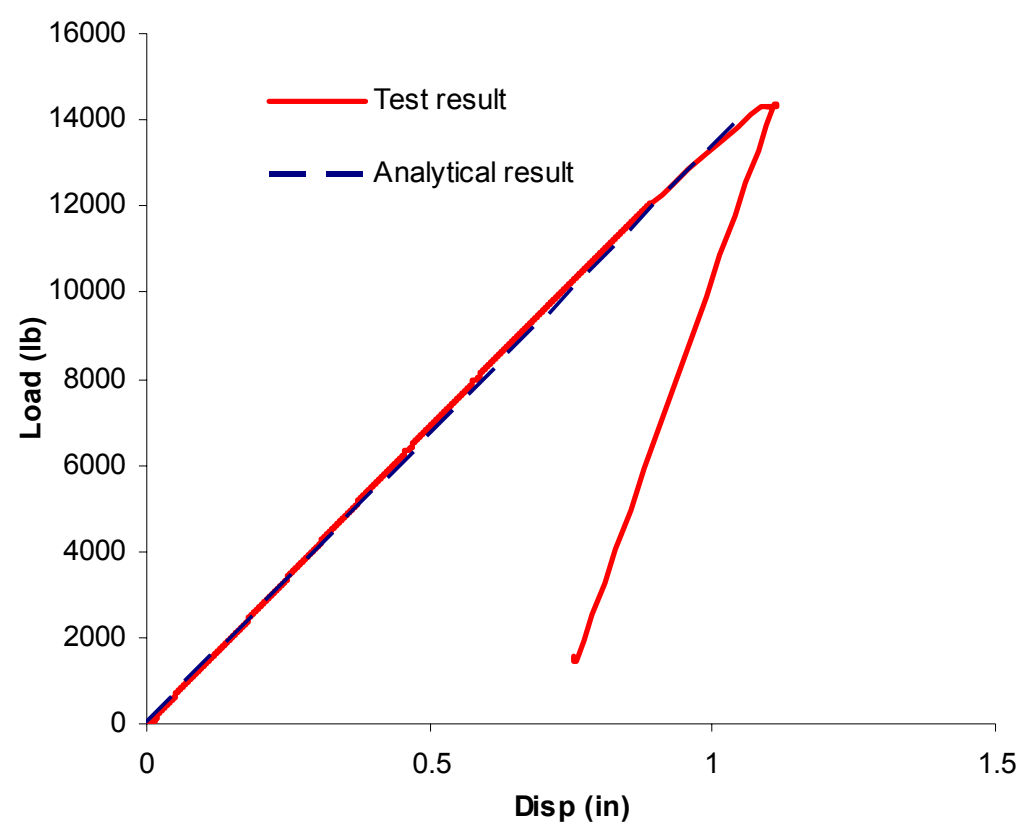

Figure 4.31 Load-displacement curve 
The model described in Section 4.5 is applied to the longitudinal specimen with excessive bonding layers. Based on basic assumptions, we have

$$
\gamma=\frac{P}{2 G_{x z} b d}
$$

where $b$ and $d$ are given in Table 4.7, and $G_{x z}$ is the equivalent shear modulus given by Eq. (4.24). For $d=2 ”$, we have

$$
G_{x z}=46,130 \quad p s i
$$

Since the shear strain of the flat panel is the same as the global shear strain, the shear stress in the flat panel can be calculated as

$$
\tau=\gamma G_{12}
$$

where $G_{12}$ is the material shear modulus from Table 3.1. The transition height $\mathrm{h}_{\mathrm{T}}$ equals to 3.84" for three bonding layers, therefore, $h<h_{T}$, and shear crushing controls. Substituting $\tau=\sigma_{6}=10,239$ psi into Eqs. (4.35) and (4.33), we can obtain the failure load as $\mathrm{P}=13,940 \mathrm{lb}$, which is in good correlation with the load from the test, $\mathrm{P}=15,840$ lb.

To further predict the response of the specimen, the following equation is employed to calculate the maximum mid-span deflections for four-point bending (Davalos et al., 2001): 


$$
\delta=\frac{23 P L^{3}}{1296 D}+\frac{P L}{6\left(\kappa G_{x z} b d\right)}
$$

where $\kappa$ is the shear correction factor and is approximately 1.0 for this study, $G_{x z}$ is the equivalent core shear stiffness, $P$ is the applied load, $L$ is the span length, and the bending stiffness $D$ is defined as

$$
D=b\left[\frac{\left(d-t_{f}\right)^{2} t_{f}}{2} E_{f}+\frac{\left(d-2 t_{f}\right)^{3}}{12} E_{c}\right]
$$

and $E_{f}, E_{c}, b, d$ and $t_{f}$ denote, respectively, facesheet bending stiffness (CADEC, Barbero, 1999), equivalent core bending stiffness (Davalos et al., 2001), beam width, beam depth and face thickness, as listed in Table 4.7.

Substituting all the values into Eq. (4.37), we can obtain

$$
\delta=7.50 \times 10^{-5} \mathrm{P}
$$

The result is illustrated in Fig. 4.31, up to $\mathrm{P}_{\max }=13,940 \mathrm{lb}$.

\subsubsection{Delamination}

Based on the discussion in Section 4.2.6.2, the design equation proposed in Section 4.5 is adopted to predict the onset of the delamination. The Flatwise Tension Test (FWT) 
(ASTM C297-94) is a standard method to measure interfacial tensile strength of honeycomb sandwich structures. A series of FWT tests were carried out by Wang (2004) and the test results are summarized in Table 4.8 .

Table 4.8 Interfacial tensile strength (from Wang, 2004)

\begin{tabular}{|c|c|}
\hline Specimen type & Interfacial tensile stress (psi) \\
\hline B1C2 & 908 \\
\hline B2C1 & 670 \\
\hline B2C2 & 714 \\
\hline B2C3 & 585 \\
\hline B3C1 & 855 \\
\hline B3C2 & 839 \\
\hline
\end{tabular}

It is expected that the specimen with more bonding layers should result in higher interfacial tensile strength, while this is not the case as observed from Table 4.8. They are somehow randomly distributed, probably due to the variance in the manufacturing process. However, it can be reasonably concluded that the interfacial tensile strength falls into the range of $500-1000$ psi.

Substituting this lower and upper bounds of interfacial tensile strength into Eqs. (4.28) and (4.33), and using the proposed method as described in Section 4.5, we can get the lowest and highest failure load for the specimen under four-point bending test, as described in Section 4.7, to be 1,549 $\mathrm{lb}$ and 3,098 lb. When comparing with the test data, with a lowest value of $3,750 \mathrm{lb}$ and a highest value of 9,310 $\mathrm{lb}$, we note that the safety factor is from 2.4 to 3.0. Therefore, the method presented in Section 4.5 provides a lower bound of the failure load. The reason for this difference is as explained in Section 4.5. 
Since the results are too conservative for design, we may, however, predict the nominal interfacial tensile strength based on the four-point bending test, as shown in Table 4.9.

Table 4.9 Nominal interfacial tensile strength

\begin{tabular}{|c|c|c|c|c|c|c|c|}
\hline & $\mathrm{B} 1 \mathrm{C} 2$ & $\mathrm{~B} 2 \mathrm{C} 2$ & $\mathrm{~B} 3 \mathrm{C} 2$ & $\mathrm{~B} 2 \mathrm{C} 1$ & $\mathrm{~B} 2 \mathrm{C} 3$ & $\mathrm{~B} 3 \mathrm{C} 1$ & $\mathrm{~B} 3 \mathrm{C} 3$ \\
\hline $\begin{array}{c}\text { Nominal interfacial } \\
\text { tensile strength }\end{array}$ & 1210 & 1754 & 2188 & 1279 & 1705 & 1713 & 3004 \\
\hline
\end{tabular}

\subsection{FE Simulation}

As concluded from the experimental results, debonding is a typical failure mode for specimens under four-point bending, where the concept of fracture mechanics should be used for FE modeling. Wang (2004) successfully developed a user-defined element using Cohesive Zone Model (CZM) and applied it to four-point bending test. To the best of the author's knowledge, this is the only work done for the analysis of HFRP sandwich structures and is listed here for completeness.

FE modeling of the four-point bending test is performed applying CZM with the mixed-mode linear-exponential constitutive law. The interfacial properties for the cohesive interface element, as listed in Table 4.10, are based on previous experimental measurements. Without experimental data for fracture toughness of Mode II and Mode III, it is assumed that $G_{c I I}=G_{c I I I}=3 G_{c I}$.

Table 4.10 Fracture toughness and interfacial strength for the four-point bending test.

\begin{tabular}{|c|c|c|c|}
\hline$G_{c \mathrm{I}}$ & $G_{c \mathrm{II}}=G_{c \mathrm{III}}$ & $\sigma_{c 3}$ & $\sigma_{c 1}=\sigma_{c 2}$ \\
\hline $25 \mathrm{lb} / \mathrm{in}$. & $75 \mathrm{lb} / \mathrm{in}$. & $800 \mathrm{psi}$ & $1500 \mathrm{psi}$ \\
\hline
\end{tabular}


A 3-D finite element model is created with ABAQUS. Due to symmetry, only half of the sandwich beam is modeled. The facesheets are modeled with shell elements, and the core is modeled entirely with solid elements. Material degradation within the facesheet-core interfaces during delamination propagation is modeled by embedding cohesive interface elements between the facesheet shell elements and core solid elements.

With resorting to $\mathrm{CZMs}$, crack initiation and growth could be successfully predicted. As shown in Fig. 4.32, the delaminated region is found to be located in the shear loading section of the beam which is consistent with the observation in the experiments. In Fig. 4.33, the finite element result of midspan deflection versus applied load is compared to experimental data of the four-point bending test. We can observe that the failure load due to facesheet delamination is accurately predicted. In the numerical simulation, severe snapback is induced right after delamination initiation, which could not be captured in the experiment when delamination propagated very quickly leading to catastrophic sudden collapse of the specimen. Because of the lack of more sophisticated numerical solution methods, the finite element analysis was terminated prematurely, since the global response was successfully captured. 


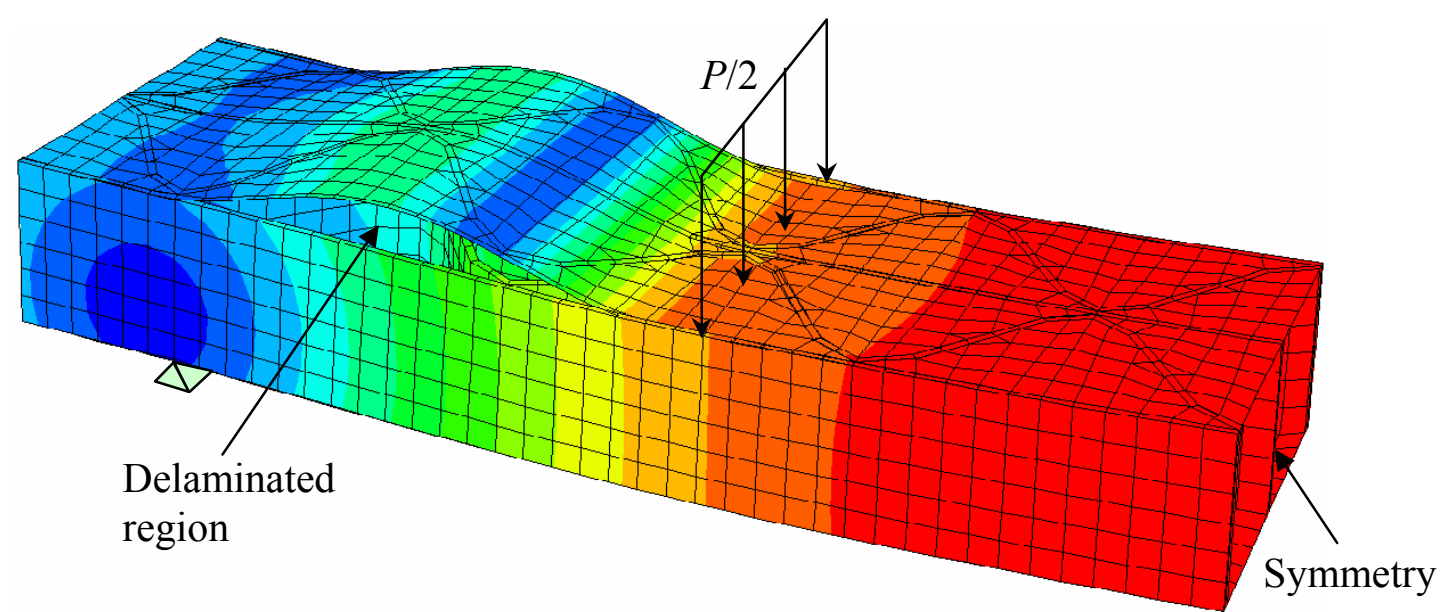

Figure 4.32 Finite element model of the four-point bending test of an HFRP sandwich panel with sinusoidal wave core configuration (from Wang, 2004).

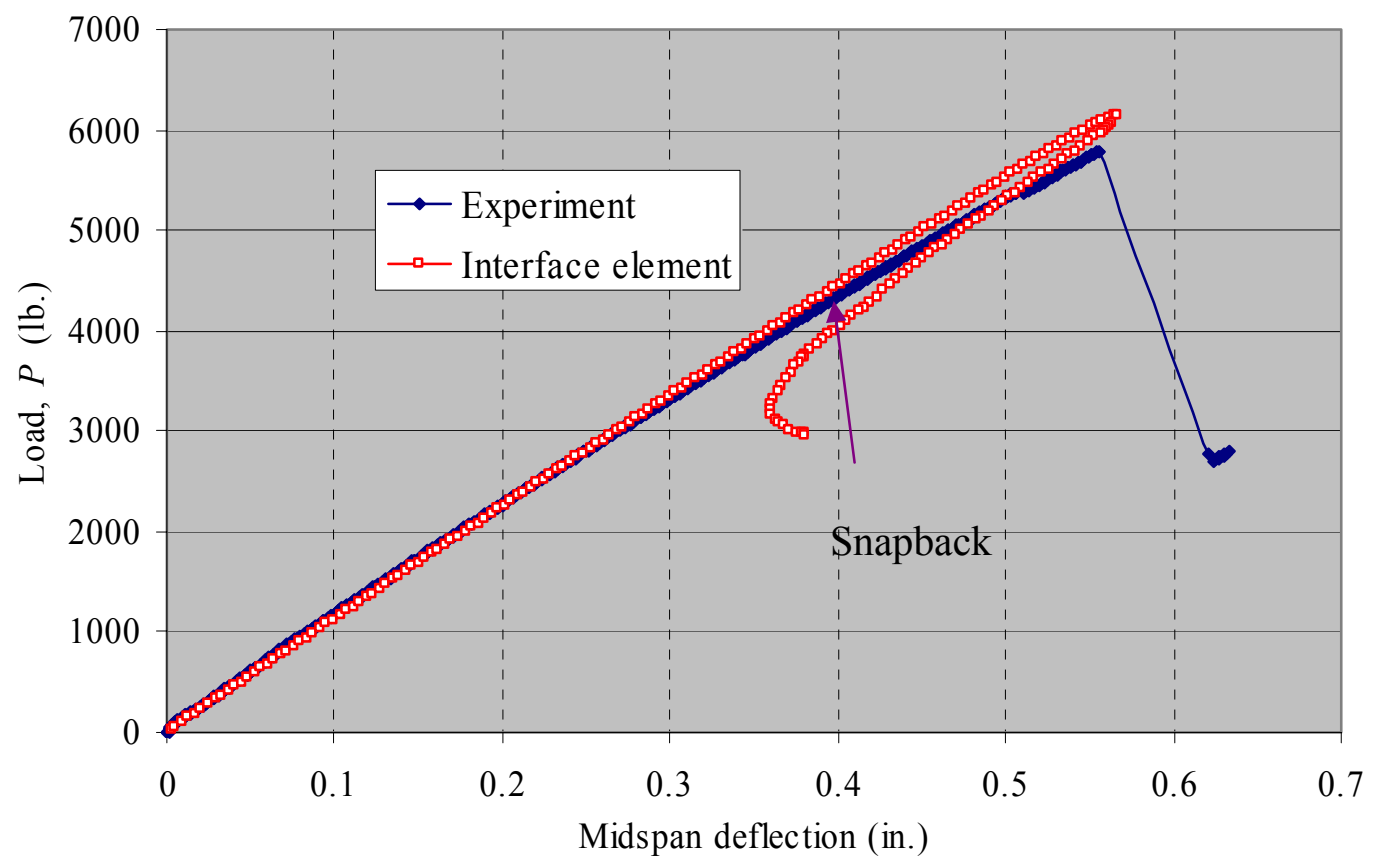

Figure 4.33 Finite element results compared to experimental data of the four-point bending test (from Wang, 2004). 


\subsection{Conclusions}

A combined analytical and experimental study of FRP sandwich panel under outof-plane shear is presented in this chapter. Analytical models, including delamination considering skin effect, shear crushing, and shear buckling are provided.

Two factors are addressed that contribute to the skin effect: shear warping and bending warping. A closed-form solution, based on proper description of displacement field at the interface, is derived considering shear warping. The accuracy of this method is verified by FE results. The FE model is then applied to study bending warping effect. The stiffness and the stress distribution subject to skin effect are presented. Critical parts are identified and suggestions for future design considerations are given. Major finding are summarized in Section 4.2.

Rayleigh-Ritz method is employed to study the shear buckling of core panels with two sides elastically restrained. Based on the analytical models, design equations are provided considering delamination, shear crushing of the core, and shear buckling.

Four-point bending tests were carried out according to ASTM standards to study shear strength and shear stiffness of the core materials. In particular, the influence of facesheet-core interface bonding effect is examined by varying the bonding layers of the specimen. Two cases of bending tests are carried out: longitudinal bending test and transverse bending test. Different failure modes were observed for different type of specimen. Design equations are used to predict the failure load due to different failure modes and good correlations are obtained. 


\section{CHAPTER 5}

\section{FACESHEET STUDY}

\subsection{Introduction}

This chapter is aimed to study the strength properties of the facesheet and to develop an optimized facesheet configuration. A progressive failure model is developed using FE method to predict the behavior of laminated composite plates up to failure, where the failure criteria are introduced through prescribed user-defined subroutines. The accuracy of the model is verified through correlations between FE results and existing experimental data. This model is then applied to carry out a parametric study on facesheet. Three variables are included: material properties, including bidirectional stitched fabrics, unidirectional layer of fiber roving and chopped strand mat; layer thickness; and layer sequences. The quality of each alternative is evaluated based on stiffness and strength performance. In order to further investigate the behavior of facesheet experimentally, coupon samples on selected configurations to evaluate compressive and bending strengths were tested in accordance with ASTM standards. The strength properties both in the longitudinal and transverse directions were evaluated. The dimensions of the coupon specimens vary for the different types of tests. The test results 
are also used to validate the progress failure model developed in this study. Through this combined experimental and analytical study, the strength properties of facesheet are obtained, which permit the optimization of facesheet design.

\subsection{Progressive Failure Model}

\subsubsection{Failure Criteria}

Various failure criteria for isotropic or composite materials have been proposed. A review of failure criteria of fibrous composite materials was given in Chapter 2. In general, the failure criteria are categorized into two groups: independent and polynomial failure criteria. The maximum stress and strain criterion belong to the first category, they are simple to apply and can tell the mode of failure, but they neglect the stress interaction. An interactive criterion such as Tsai-Wu, Hoffman, or Hill, includes stress interaction in the failure mechanism, but it does not tell the mode of failure, and it requires some efforts to determine parameters such as $F_{12}$ in Tsai-Wu criterion. Among others, Hashin (1980) provided a three dimensional failure criterion, which includes fiber tension, fiber compression, matrix tension, and matrix compression. This criterion not only considers the stress interaction, but provides the failure mode. Therefore, it is widely used (Kroll and Hufenbach, 1997; Spottswood and Palazotto, 2001) and is adopted in this study. However, Hashin (1980) did not specify the delamination criterion, which becomes significant when the laminate fails due to interlaminar shear failure, as will be shown in Section 5.3. This issue was recently addressed by Elawadly (2003). Fortunately, Lee (1982) further proposed a delamination mode in his 3-D analysis, and is adopted in this study as an addition to Hashin's failure criterion, as shown below. 
For a plane stress problem, when considering the transverse shear components, the failure criteria take the form (Hashin, 1980; Lee, 1982):

Tensile fiber mode:

$$
\left(\frac{\sigma_{11}}{X_{T}}\right)^{2}+\left(\frac{\sigma_{12}^{2}+\sigma_{13}^{2}}{S_{12}^{2}}\right)^{2}=1 \quad \sigma_{11}>0
$$

Compressive fiber mode:

$$
\sigma_{11}=X_{C} \quad \sigma_{11}<0
$$

Tensile matrix mode:

$$
\left(\frac{\sigma_{22}}{Y_{T}}\right)^{2}+\frac{\sigma_{23}^{2}}{S_{23}^{2}}+\left(\frac{\sigma_{12}^{2}+\sigma_{13}^{2}}{S_{12}^{2}}\right)=1 \quad \sigma_{22}>0
$$

Compressive matrix mode:

$$
\left[\left(\frac{X_{c}}{2 S_{23}}\right)^{2}-1\right] \frac{\sigma_{22}}{Y_{C}}+\left(\frac{\sigma_{22}}{2 S_{23}}\right)^{2}+\frac{\sigma_{23}^{2}}{S_{23}^{2}}+\frac{\sigma_{12}^{2}+\sigma_{13}^{2}}{S_{12}^{2}}=1 \quad \sigma_{22}<0
$$


Transverse shear mode:

$$
\frac{\sigma_{13}^{2}+\sigma_{23}^{2}}{S_{D S}^{2}}=1
$$

where

$\sigma_{i j}=$ Stress tensor; $X_{T}=$ tensile failure stress in fiber direction; $X_{C}=$ compressive failure stress in fiber direction; $Y_{T}=$ tensile failure stress transverse to fiber direction; $Y_{C}=$ compressive failure stress transverse to fiber direction; $S_{12}=$ axial failure shear; $S_{23}$ $=$ transverse failure shear; and $S_{D S}=$ interlaminar failure shear.

The material state corresponding to each type of damage is listed in Table 5.1.

Table 5.1 Material state

\begin{tabular}{|c|c|c|c|c|c|c|}
\hline Material State & \multicolumn{6}{|c|}{ Elastic properties } \\
\hline No failure & $\mathrm{E}_{\mathrm{x}}$ & $\mathrm{E}_{\mathrm{y}}$ & $v_{\mathrm{xy}}$ & $\mathrm{G}_{\mathrm{xy}}$ & $\mathrm{G}_{\mathrm{xz}}$ & $\mathrm{G}_{\mathrm{yz}}$ \\
\hline Matrix failure & $\mathrm{E}_{\mathrm{x}}$ & 0 & 0 & 0 & 0 & 0 \\
\hline Fiber failure & 0 & 0 & 0 & 0 & 0 & 0 \\
\hline Matrix/fiber failure & 0 & 0 & 0 & 0 & 0 & 0 \\
\hline Transverse shear damage & $\mathrm{E}_{\mathrm{x}}$ & $\mathrm{E}_{\mathrm{y}}$ & $v_{\mathrm{xy}}$ & $\mathrm{G}_{\mathrm{xy}}$ & 0 & 0 \\
\hline Matrix failure/shear damage & $\mathrm{E}_{\mathrm{x}}$ & 0 & 0 & 0 & 0 & 0 \\
\hline Fiber failure/shear damage & 0 & 0 & 0 & 0 & 0 & 0 \\
\hline All damage modes & 0 & 0 & 0 & 0 & 0 & 0 \\
\hline
\end{tabular}

\subsubsection{Progressive Failure Analysis}

A lot of research has been done in the area of progressive failure analysis. Mostly commonly used are 2-D (Kim et al., 1996) and 3-D analyses (Reddy and Reddy, 1993). For 2-D analysis, based on plane stress assumption, the transverse shear stresses, $\sigma_{13}$ and 
$\sigma_{23}$, and normal stress, $\sigma_{33}$, are neglected. As a result, the failure mode of delamination cannot be considered. 3-D progressive failure analysis was successfully developed by several researchers. However, the disadvantage is apparent. Take a 32-layer laminate as an example, the element will be 32 times of that in 2-D modeling, resulting in a cumbersome work both for modeling and computation, which hampers its use for a parametric study. Therefore, it is the objective of this study to develop a model that uses 2-D element and can still predict the delamination failure. Since $\sigma_{33}$ is negligible considering the thickness-to-length ratio for each layer, only $\sigma_{13}$ and $\sigma_{23}$ should be considered for delamination. In ABAQUS (2002), transverse shear stresses are not readily available in the output stress components for a shell element. Instead, they are stored in the result file as TSHR13 and TSHR23. Therefore, a user-defined subroutine is firstly employed to retrieve the transverse shear stresses from the result file. Combining with another subroutine to implement the failure criterion displayed in the previous section, the progressive failure analysis can be carried out.

Due to the nonlinearity after the first-ply failure, displacement control is adopted with the following algorithm:

(1) Obtain stresses for each material point from previous increment, and retrieve the transverse shear components from the result file;

(2) Use Hashin's failure criterion to calculate failure index;

(3) Update the field variable according to Table 5.1;

(4) Increase the displacement by a given time step;

(5) Repeat steps 1 through 4 until ultimate failure is reached. 


\subsection{Verification Study ${ }^{*}$}

Grief and Chapon (1993) conducted three-point bending tests of composite beams made of AS4/3502 graphite-epoxy pre-preg tape; the material properties and strength parameters of the test-specimens are listed in Table 5.2. Five different laminate types were tested, with two specimens for each type, and the test beam specifications are given in Table 5.3. The reliability of the results was proved by Kim, Davalos and Barbero (1996). Therefore, it is adopted herein for verification purpose.

First of all, the convergence study is carried out and then the mesh is defined as 30x6 elements. Shell element (S4) in ABAQUS is employed. The predicted vs. experimental load-displacement diagrams for selected graphite-epoxy laminates are shown in Fig. 5.1, where good agreement can be observed, although some discrepancies for post failure paths can be noticed. Figure 5.2 plots the curves of load and displacement from FE prediction and test results, illustrating a good correlation.

It is worthy to point out that, as concluded by Greif and Chapon (1993), beam type B failed due to delamination, which can be easily understood through a free edge analysis, and other types followed a roughly progressive failure manner. As interlaminar shear strength, $\mathrm{S}_{\mathrm{DS}}$, is not available in the literature, a value of $0.011 \mathrm{GPa}$ is assumed, as shown in Table 5.2. From the FE analysis, a higher shear stress results at the $0^{\circ} / 90^{\circ}$ interfaces. The failure load of type $\mathrm{B}$ is highly dependent on the value of $\mathrm{S}_{\mathrm{DS}}$, while other types of laminate are not affected too much, which corroborates the accuracy of the FE model developed in this study.

\footnotetext{
${ }^{*}$ For verification purpose, metric unit is used in this section.
} 
Table 5.2 Material properties and strength parameters

\begin{tabular}{|c|c|}
\hline Material Properties & Elastic properties \\
\hline $\mathrm{E}_{1}=141.2 \mathrm{GPa}$ & $\mathrm{X}_{\mathrm{T}}=2.343 \mathrm{GPa}$ \\
$\mathrm{E}_{2}=11.5 \mathrm{GPa}$ & $\mathrm{X}_{\mathrm{c}}=1.723 \mathrm{GPa}$ \\
$\mathrm{G}_{12}=6.0 \mathrm{GPa}$ & $\mathrm{Y}_{\mathrm{T}}=0.051 \mathrm{GPa}$ \\
$\mathrm{v}_{12}=0.3$ & $\mathrm{Y}_{\mathrm{c}}=0.223 \mathrm{GPa}$ \\
& $\mathrm{S}_{12}=2.343 \mathrm{GPa}$ \\
& $\mathrm{S}_{\mathrm{DS}}=0.011 \mathrm{GPa}$ (assumed) \\
\hline
\end{tabular}

Table 5.3 Beam specifications

\begin{tabular}{|c|c|c|c|c|c|}
\hline Laminate & Lay-up & $\begin{array}{c}\text { No. of } \\
\text { Plies }\end{array}$ & $\begin{array}{c}\text { Length } \\
(\mathbf{m m})\end{array}$ & $\begin{array}{c}\text { Width } \\
(\mathbf{m m})\end{array}$ & $\begin{array}{c}\text { Thickness } \\
(\mathbf{m m})\end{array}$ \\
\hline A1 & {$\left[90_{8} / 0_{8}\right]_{\mathrm{s}}$} & 32 & 139.7 & 25.84 & 4.468 \\
\hline A2 & {$\left[90_{8} / 0_{8}\right]_{\mathrm{s}}$} & 32 & 152.4 & 25.65 & 4.547 \\
\hline B1 & {$\left[0_{8} / 90_{8}\right]_{\mathrm{s}}$} & 32 & 127.0 & 24.13 & 4.597 \\
\hline B2 & {$\left[0_{8} / 90_{8}\right]_{\mathrm{s}}$} & 32 & 152.4 & 24.69 & 4.674 \\
\hline C1 & {$\left[(0 / 90)_{8}\right]_{\mathrm{s}}$} & 32 & 152.4 & 25.65 & 4.470 \\
\hline C2 & {$\left[(0 / 90)_{8}\right]_{\mathrm{s}}$} & 32 & 152.4 & 24.33 & 4.470 \\
\hline D1 & {$\left[(45 / 0 /-45)_{5}\right]_{\mathrm{s}}$} & 30 & 152.4 & 24.26 & 4.166 \\
\hline D2 & {$\left[(45 / 0 /-45)_{5}\right]_{\mathrm{s}}$} & 30 & 152.4 & 24.26 & 4.166 \\
\hline E1 & {$\left[(0 / 45 / 0 /-45)_{3} / 90 / 0 / 0_{1 / 2}\right]_{\mathrm{s}}$} & 29 & 152.4 & 24.49 & 4.039 \\
\hline E2 & {$\left[(0 / 45 / 0 /-45)_{3} / 90 / 0 / 0_{1 / 2}\right]_{\mathrm{s}}$} & 29 & 152.4 & 25.30 & 4.039 \\
\hline
\end{tabular}




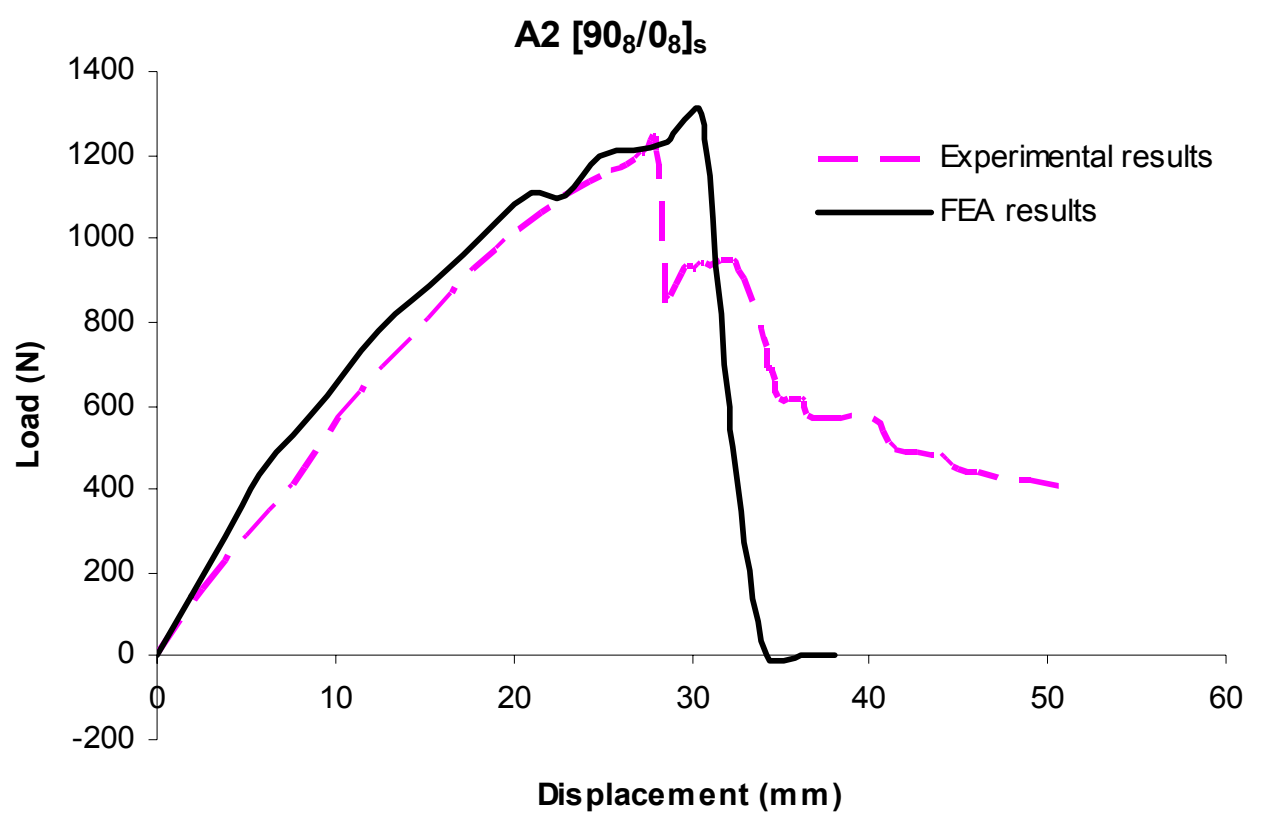

(a) Laminate A2

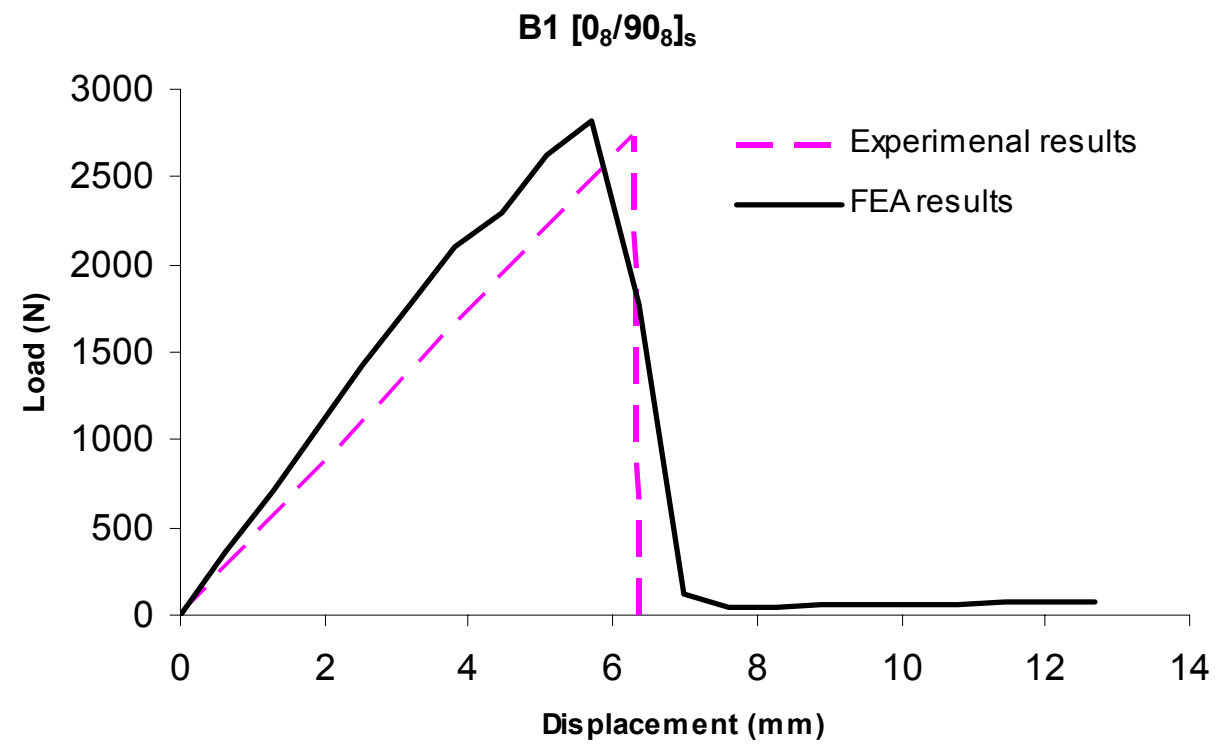

(b) Laminate B1 


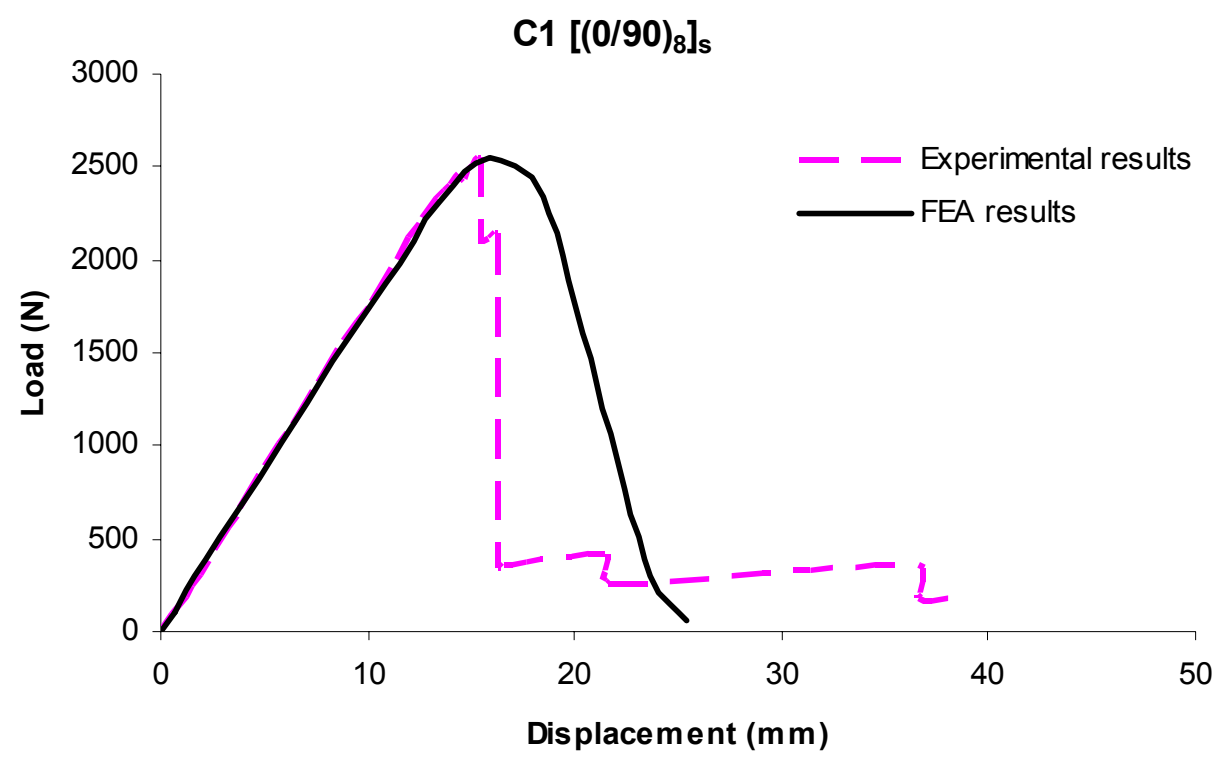

(c) Laminate C1

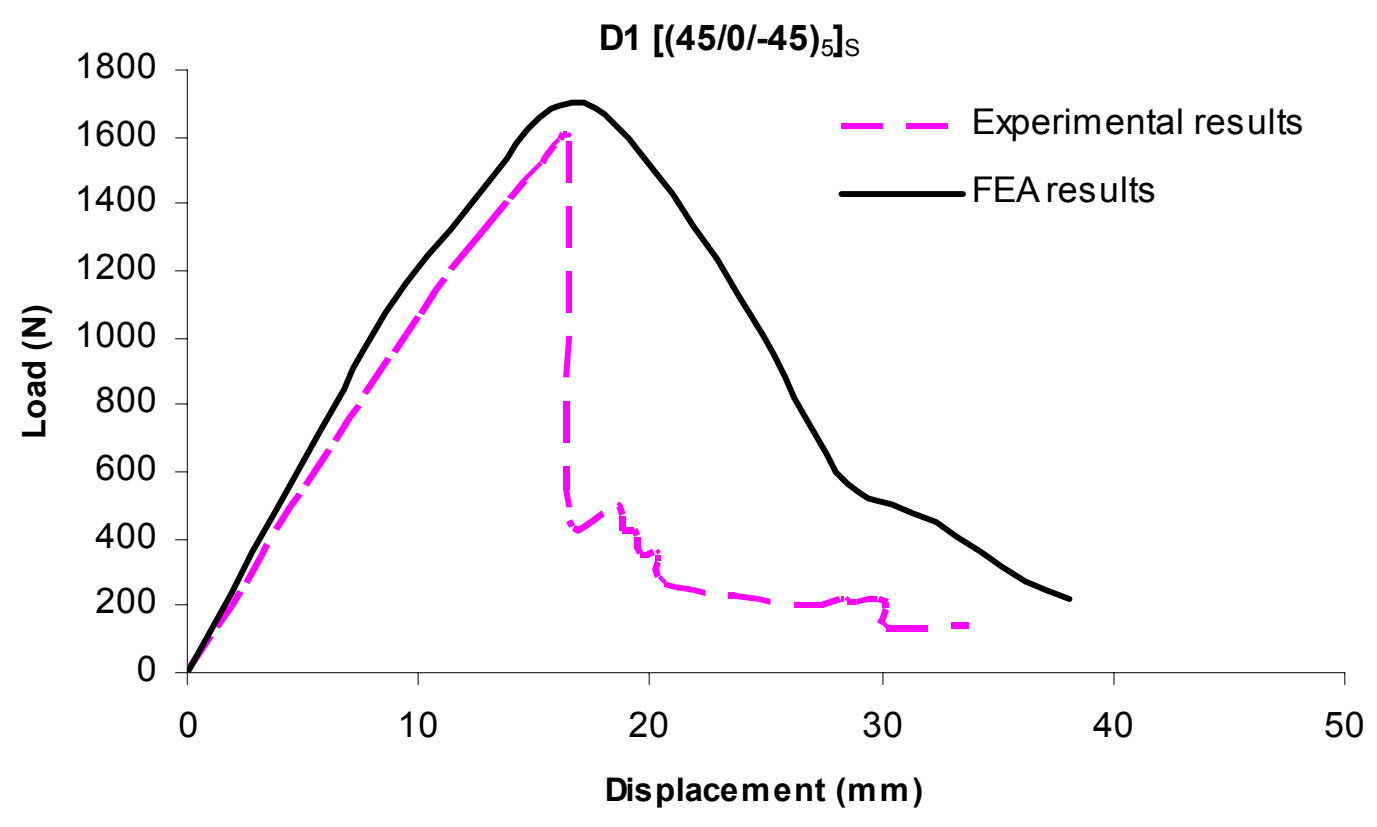

(d) Laminate D1

Figure 5.1 Load-displacement paths 


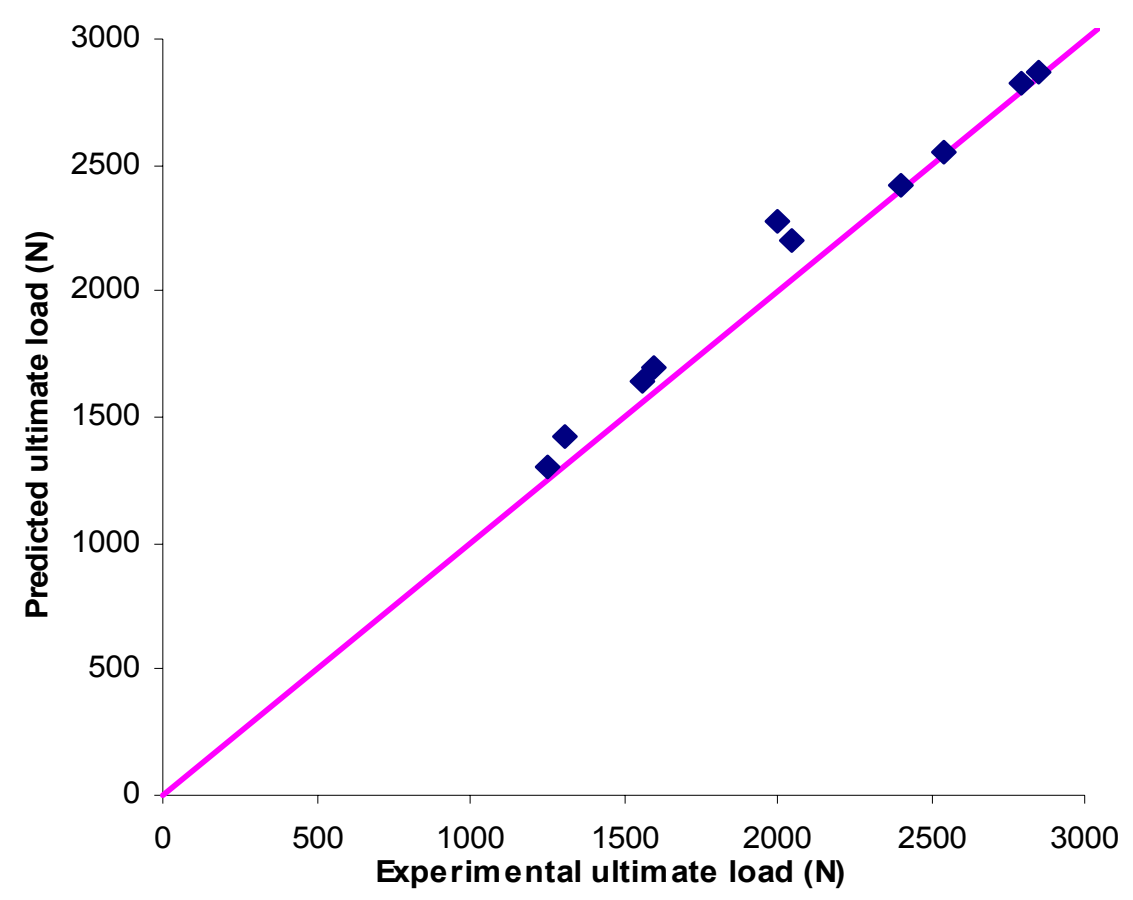

(a) Ultimate load

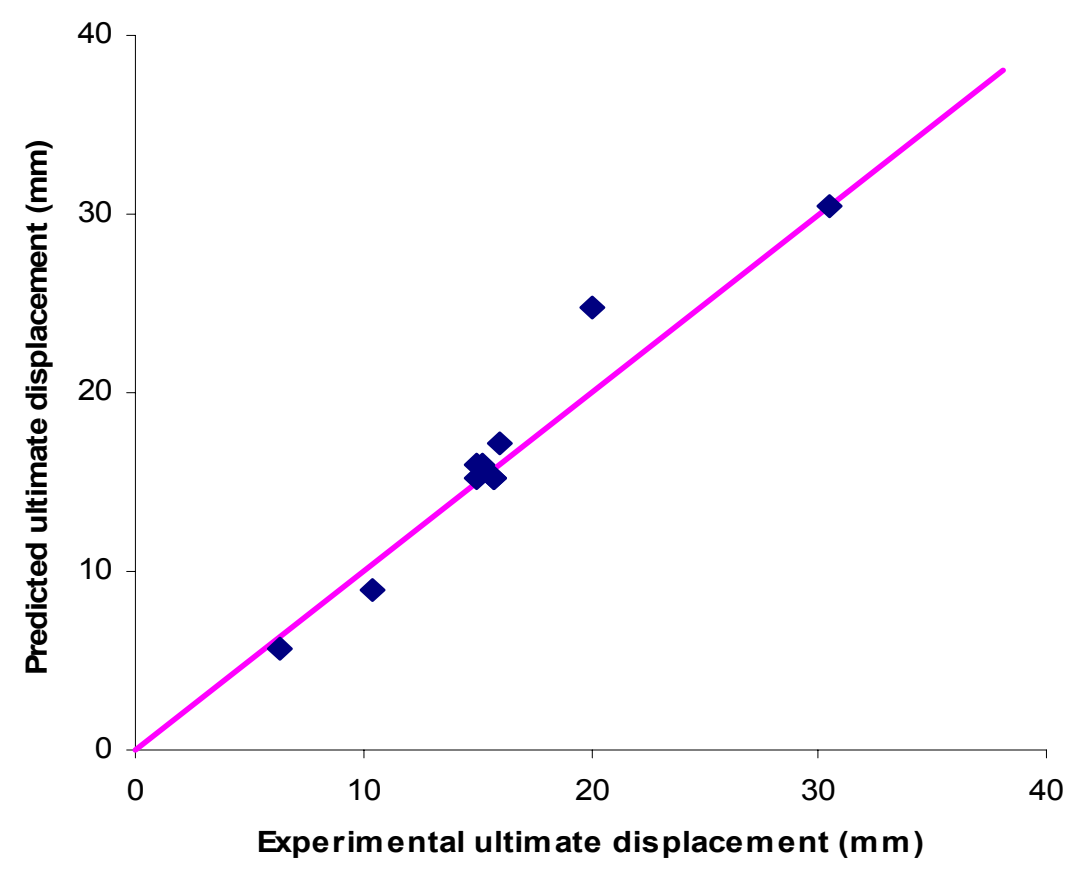

(b) Ultimate displacement

Figure 5.2 Comparison of ultimate load and displacement 


\subsection{Parametric Study on Facesheet}

In a sandwich panel, the two stiff facesheets carry the membrane force and the light weight core resists the out-of-plane shear. As composite action is not considered for an FRP sandwich bridge deck panel, the top and bottom facesheet are respectively subjected to compression and tension in equal magnitude, or vice versa, depending on whether the panel is in positive or negative bending region. Therefore, the in-plane force is the major concern when designing a facesheet. Since the facesheet can be characterized by longitudinal and transverse direction, four combinations should be considered, namely, tension along longitudinal direction (TL), compression along longitudinal direction (CL), tension along transverse direction (TT), and compression along transverse direction (CT).

Three variables are included in the parametric study: material properties, including bidirectional stitched fabrics, unidirectional layer of fiber roving and chopped strand mat; layer thickness; and layer sequences, as listed Table in 5.4. It is noted that laminate \#7 is the facesheet being used in industry. The material properties given in Table 5.5 are obtained from a previous study by Davalos et al. (2001). The strength parameters given in Table 5.6 are calculated using CADEC (Barbero, 1999). As delamination is not a concern for all laminates, $\mathrm{S}_{\mathrm{DS}}=\mathrm{S}_{12}$ is assumed for all the calculations. 
Table 5.4 Laminate configuration

\begin{tabular}{|c|c|c|c|c|c|c|c|}
\hline Laminate & $\# 1$ & $\# 2$ & $\# \mathbf{3}$ & $\# 4$ & $\# 5$ & \#6 & $\# 7$ \\
\hline & $1-\mathrm{Bi}^{\mathrm{a}}$ & $1-\mathrm{Bi}$ & 1-Bi & $1-\mathrm{Bi}$ & 6-Bi & 1-Bi & 1-Bi \\
\hline & 2-Uni ${ }^{\mathrm{b}}$ & 1-ChSM & 2-ChSM & 1-ChSM & 4-ChSM & 6-Uni & 8-Uni \\
\hline & 1-Bi & 1-Bi & 1-Bi & 4-Uni & & 1-Bi & 1-Bi \\
\hline & 2-Uni & 1-ChSM & 2-ChSM & 1-ChSM & & 2-ChSM & 2-ChSM \\
\hline & 1-Bi & 1-Bi & 1-Bi & 1-Bi & & & \\
\hline & 4-ChSM ${ }^{\mathrm{c}}$ & 1-ChSM & 4-ChSM & 4-ChSM & & & \\
\hline & & 1-Bi & & & & & \\
\hline & & 4-ChSM & & & & & \\
\hline Thickness (in.) & 0.49 & 0.52 & 0.51 & 0.52 & 0.52 & 0.43 & 0.51 \\
\hline
\end{tabular}

${ }^{\mathrm{a} B i}$ CM 3205;

${ }^{b}$ Uni: UM 1810;

c1-ChSM: nominal weight $=1.5 \mathrm{oz} / \mathrm{ft}^{2}$.

Table 5.5 Material properties

\begin{tabular}{|c|c|c|c|c|c|c|}
\hline Type & $\begin{array}{c}E_{1} \\
\left(x_{10}{ }^{6} \text { psi) }\right.\end{array}$ & $\begin{array}{c}E_{2} \\
\left(\times 10^{6} \text { psi) }\right.\end{array}$ & $\begin{array}{c}G_{12} \\
\left(x^{6} 0^{6} \text { psi) }\right.\end{array}$ & $\begin{array}{c}\mathbf{G}_{23} \\
\left(\mathbf{x 1 0}^{6} \mathrm{psi}\right)\end{array}$ & $v_{12}$ & $v_{23}$ \\
\hline $\begin{array}{c}\text { CM } 3205 \\
0^{\circ} / 90^{\circ}\end{array}$ & 4.025 & 1.160 & 0.447 & 0.418 & 0.295 & 0.39 \\
\hline CM 3205 CSM & 1.710 & 1.710 & 0.611 & 0.342 & 0.402 & 0.4 \\
\hline UM $18100^{\circ}$ & 4.360 & 1.240 & 0.479 & 0.447 & 0.293 & 0.386 \\
\hline UM 1810 CSM & 2.310 & 2.31 & 0.819 & 0.429 & 0.409 & 0.388 \\
\hline $\begin{array}{c}\text { Bond layer } \\
\text { ChSM }\end{array}$ & 1.41 & 1.41 & 0.507 & 0.307 & 0.394 & 0.401 \\
\hline
\end{tabular}


Table 5.6 Strength parameters (psi)

\begin{tabular}{|c|c|c|c|c|c|c|}
\hline Type & $\mathbf{X}_{\mathbf{T}}$ & $\mathbf{X}_{\mathbf{C}}$ & $\mathbf{Y}_{\mathbf{T}}$ & $\mathbf{Y}_{\mathbf{C}}$ & $\mathbf{S}_{\mathbf{1 2}}$ & $\mathbf{S}_{\mathbf{2 3}}$ \\
\hline $\begin{array}{c}\text { CM 3205 } \\
0^{\circ} / 90^{\circ}\end{array}$ & 194496 & 58595 & 6672 & 9572 & 6672 & 6672 \\
\hline $\mathrm{CM} 3205 \mathrm{CSM}^{\circ}$ & 22046 & 22046 & 22046 & 22046 & 11023 & 12038 \\
\hline UM 1810 0 & 210595 & 59320 & 6672 & 9427 & 6672 & 6672 \\
\hline UM 1810 CSM & 23061 & 23061 & 23061 & 23061 & 11458 & 12038 \\
\hline $\begin{array}{c}\text { Bond layer } \\
\text { ChSM }\end{array}$ & 21321 & 21321 & 21321 & 21321 & 10588 & 12038 \\
\hline
\end{tabular}

Either tensile or compressive loads, acting either along longitudinal or transverse directions, are applied to simulated specimens of 8 "x2" of laminates with different configurations. Typical curves for a balanced laminate (\#3) and an unbalanced laminate (\#7) are respectively shown in Fig. 5.3 and Fig. 5.4, where we can see that the compressive load is more critical for both cases. Apparently \#7 is not optimized as the tensile strength along the longitudinal direction is much higher than the compressive strength, whereas the compressive load controls the final design.

Since the axial load is mainly carried out along the longitudinal direction, CL case is further considered for all configurations. Load-displacement curves are illustrated in Fig. 5.5. CL strength for \#7 is 31,873 psi and the normalized strength based on \#7 is shown in Fig. 5.6. To illustrate the change of stiffness for each laminate, a static analysis is carried out for a patch load of 20 kips acting at the center of an 8'x8' sandwich panel with 8 " thick core. The deflection of \#7 is 0.1 " and the normalized defection based on \#7 is shown in Fig. 5.7. 
From the analysis above, it is shown that when ChSM is introduced into the facesheet, the strength is not affected much while the stiffness drops a lot. Consider \#3 as an example, the strength is $9 \%$ lower and the deflection is $36 \%$ higher than those of $\# 7$. However, the deflection for \#3, which is $\mathrm{L} / 700$, where $\mathrm{L}$ is the span of the deck, is still in the acceptable range.

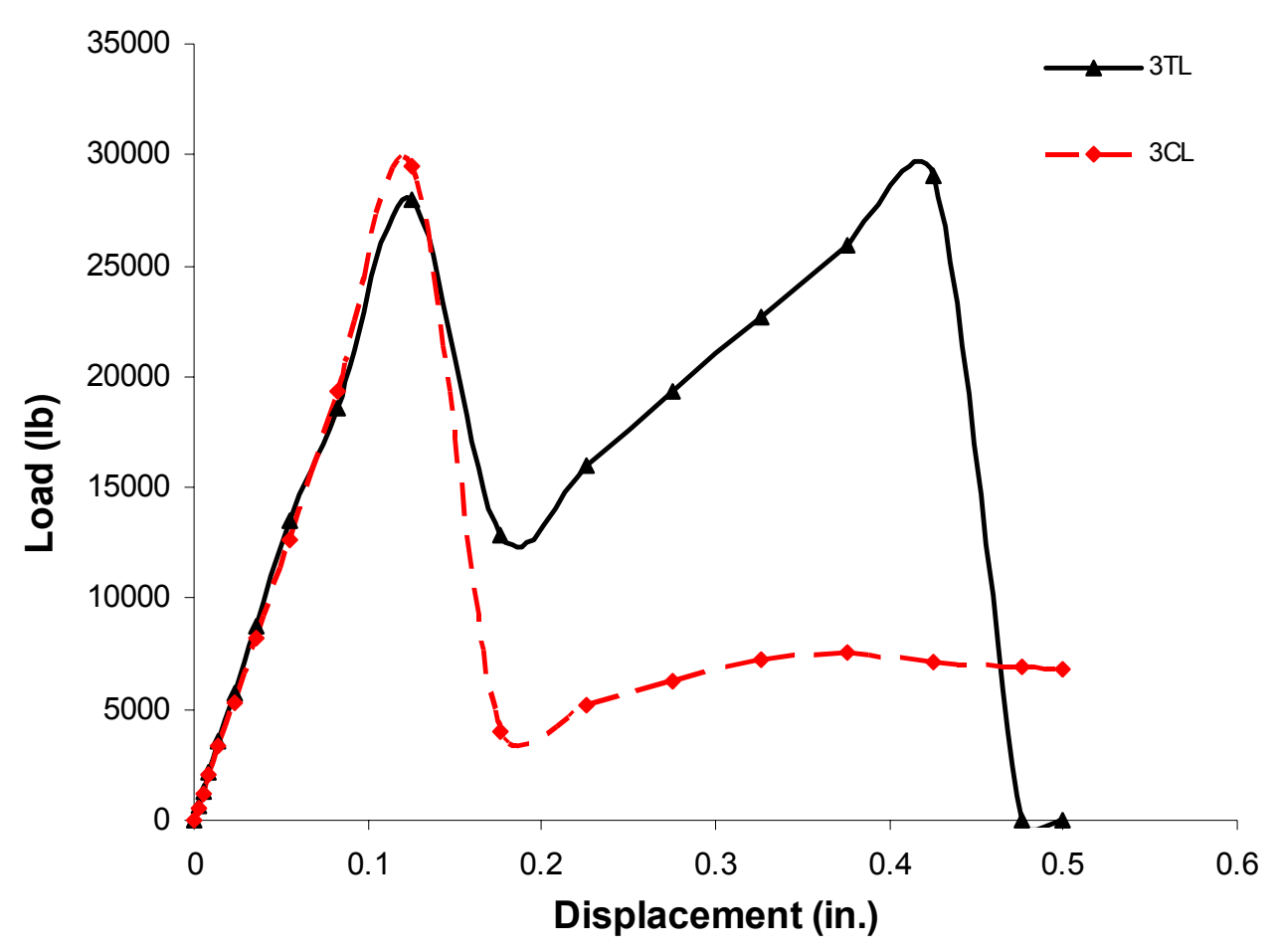

Figure 5.3 Load-displacement curve for \#3 


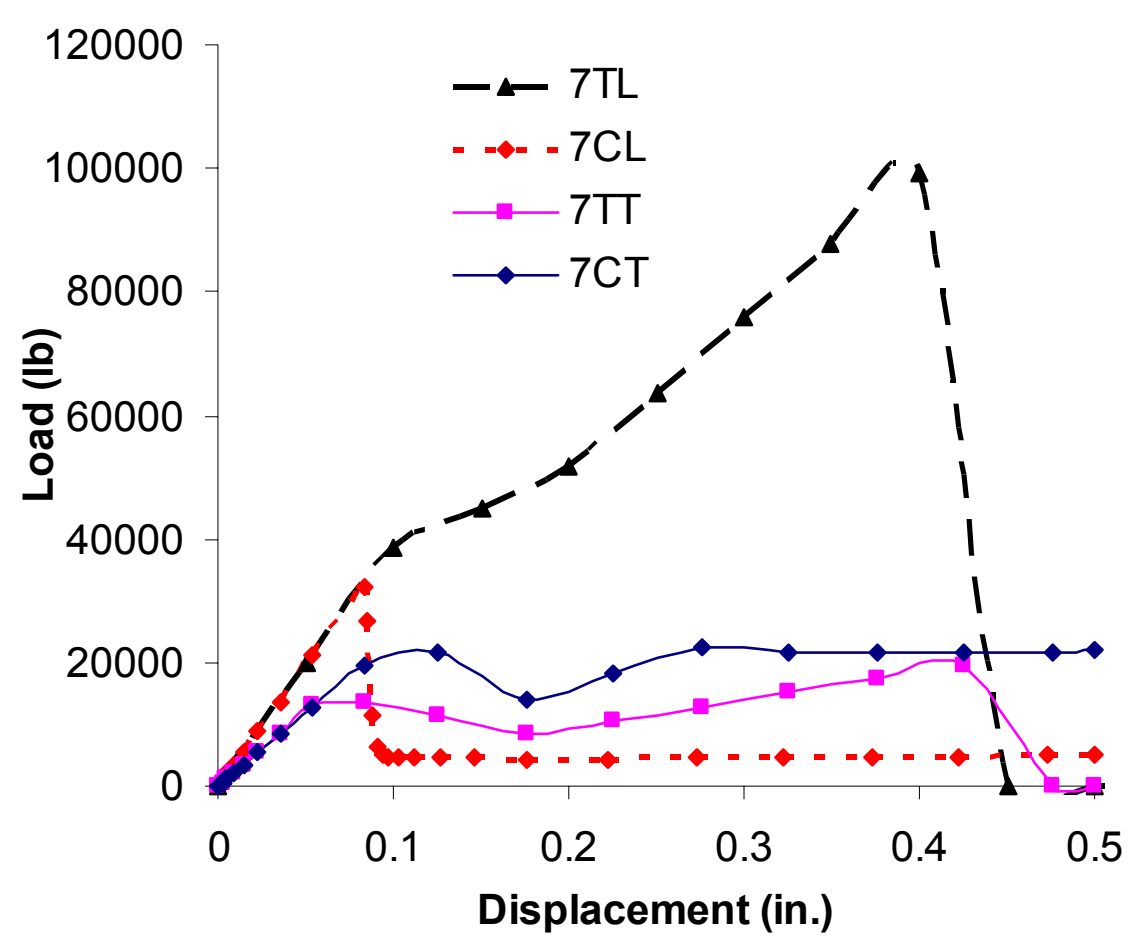

Figure 5.4 Load-displacement curve for \#7 


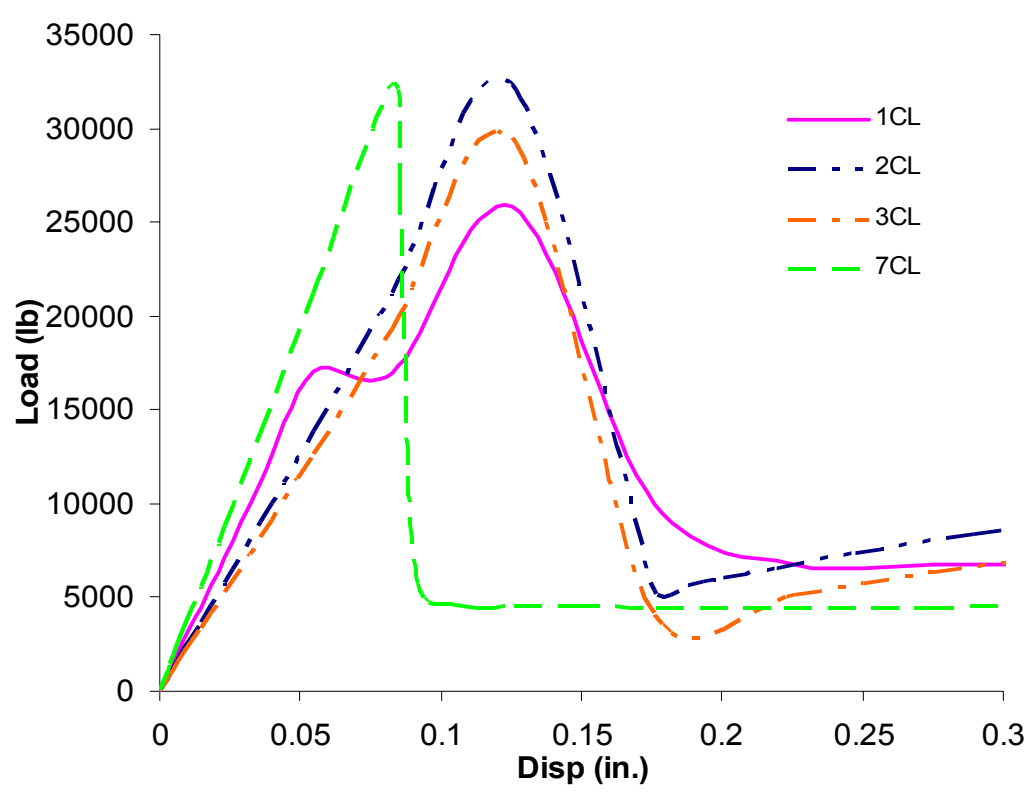

(a)

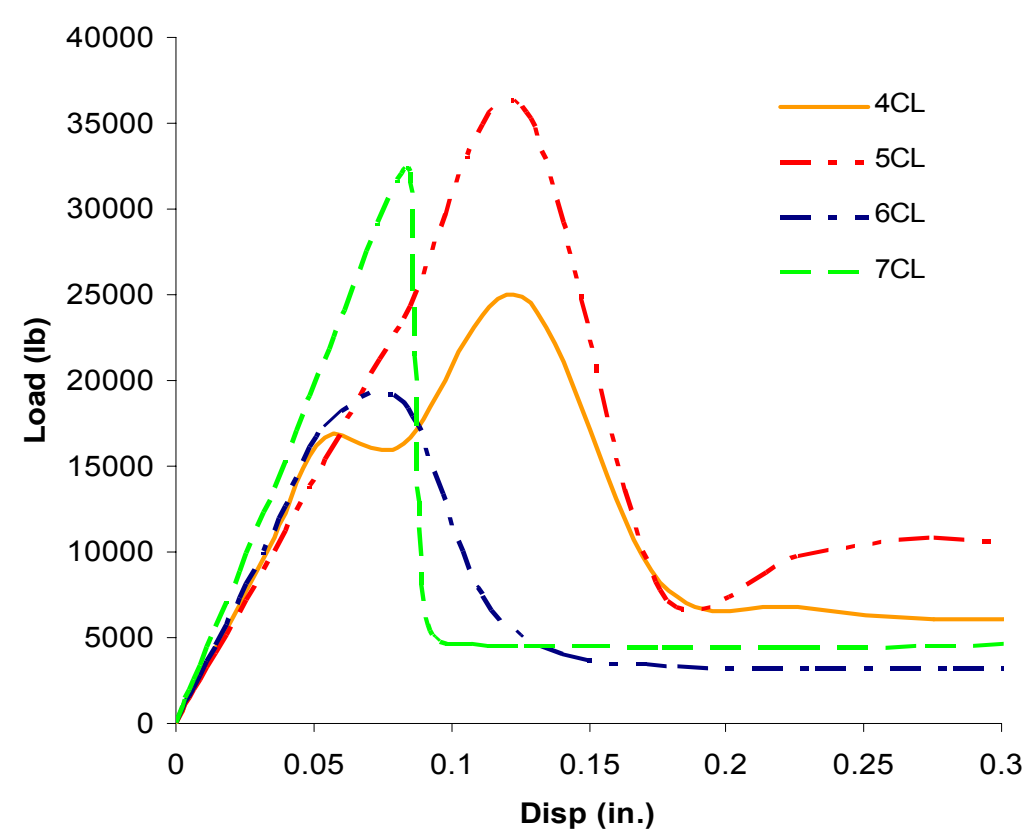

(b)

Figure 5.5 Load-displacement curves for CL 


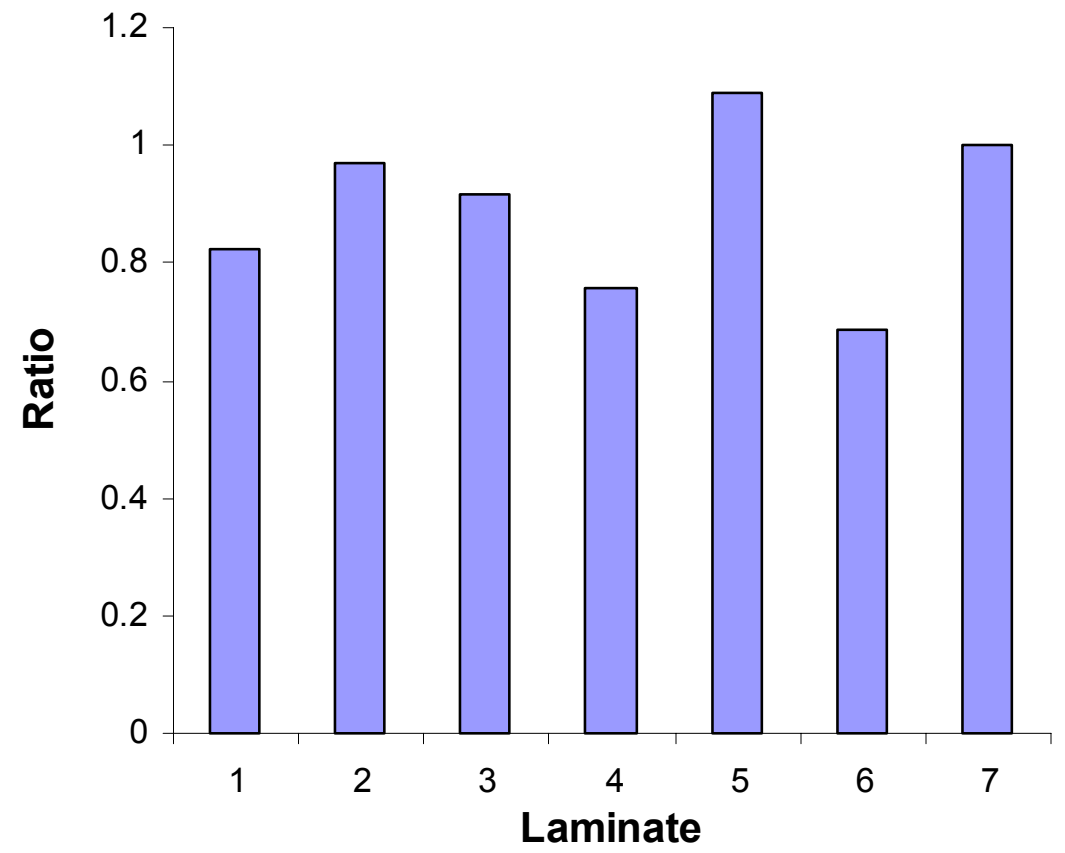

Figure 5.6 Normalized failure strength

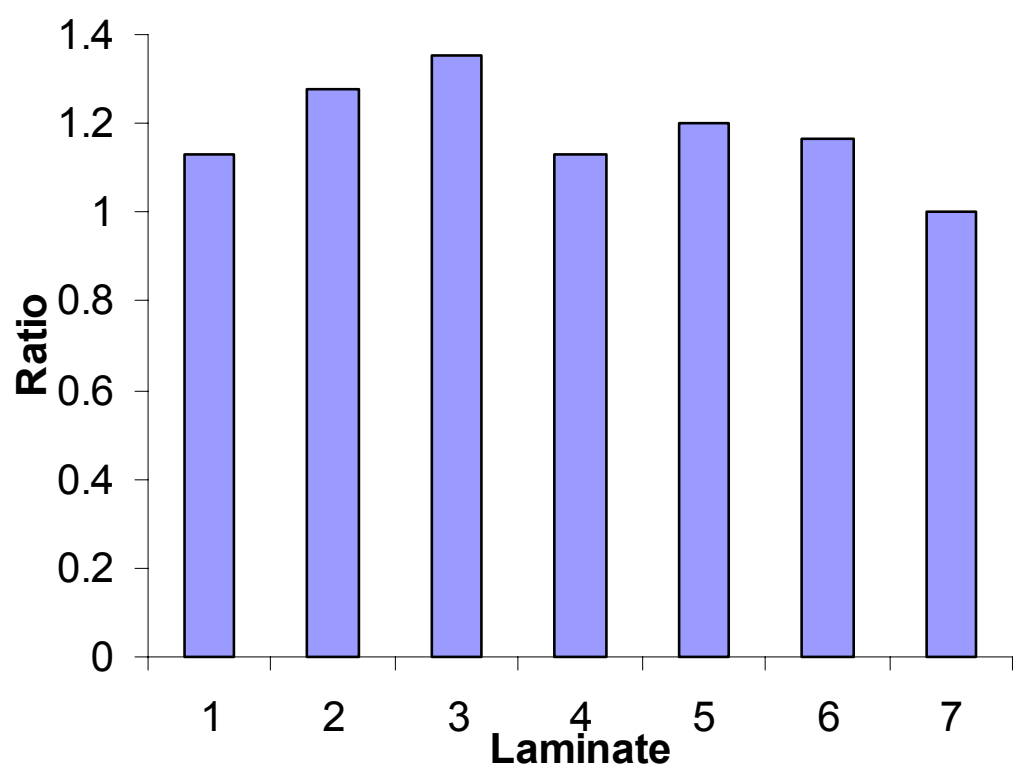

Figure 5.7 Normalized deflection 


\subsection{Experimental Investigation}

Table 5.7 Plate configurations

\begin{tabular}{|c|c|c|c|}
\hline & $\begin{array}{c}\text { Laminate } 1(\# 7)^{*} \\
\text { (current) }\end{array}$ & Laminate $2(\# 3)^{*}$ & Laminate $3(\# 5)^{*}$ \\
\hline & $\begin{array}{c}2 \text { layers } 3.0 \text { oz. ChSM } \\
1 \text { layer Bi-axial } \\
9 \text { layers Uni-axial } \\
1 \text { layer Bi-axial }\end{array}$ & $\begin{array}{c}2 \text { layers } 3.0 \mathrm{oz} . \mathrm{ChSM} \\
1 \text { layer Bi-axial } \\
1 \text { layer } 3.0 \mathrm{oz} . \mathrm{ChSM} \\
1 \text { layer Bi-axial } \\
1 \text { layer } 3.0 \mathrm{oz} . \mathrm{ChSM} \\
1 \text { layer Bi-axial } \\
1 \text { layer } 3.0 \mathrm{oz} . \mathrm{ChSM} \\
1 \text { layer Bi-axial } \\
1 \text { layer } 3.0 \mathrm{oz} . \mathrm{ChSM}\end{array}$ & $\begin{array}{c}2 \text { layers } 3.0 \text { oz. ChSM } \\
8 \text { layers Bi-axial }\end{array}$ \\
\hline Thickness & $0.59 "$ & $0.65^{\prime \prime}$ & $0.56 "$ \\
\hline
\end{tabular}

*: The number used in parametric study.

Note: Bi-axial: $\quad$ CDM 3208

Uni-axial: $\quad$ CM 1708

ChSM: $\quad$ Chopped Strand Mat

Based on the results from parametric study, three configurations are selected to further study the strength behavior of facesheet, as shown in Table 5.7. Three-point bending and compression tests are carried out. Since Laminate 1 is not balanced, the tests are carried out both along longitudinal and transverse directions, resulting in four different types, labeled as 1L, 1T, 2 and 3. For completeness, shear test results and stiffness for facesheet laminates are provided in Appendix D and E, respectively. 


\subsubsection{Three-Point Bending Test}

\subsubsection{Experimental Setup}

Three-point bending test was chosen for the following reasons: (1) the testing apparatus has a simple test setup, no complicated hardware or equipment required, and (2) the results are relatively easy to interpret. As pointed out by Greif and Chapson (1993), three-point bending test usually yields good results for material characterization of composites, such as laminate moduli of elasticity, laminate stresses, etc. The test setup is shown in Fig. 5.8, which consists of a simply supported beam between two supports with the load applied at the mid span. The photo of the setup is shown in Fig. 5.9. The dimension of the specimen is $15 "$ long and 2" wide. According to ASTM standards (ASTM D790-99), the span is chosen to be 12". Four different types were tested, with five specimens for each type. The tests were carried out in an MTS machine. Strain gage was bonded at the bottom of mid-span, the displacement and load were recorded using the internal displacement transducer and load cell. The loading rate was controlled at $0.33 \% / \mathrm{min}$. 


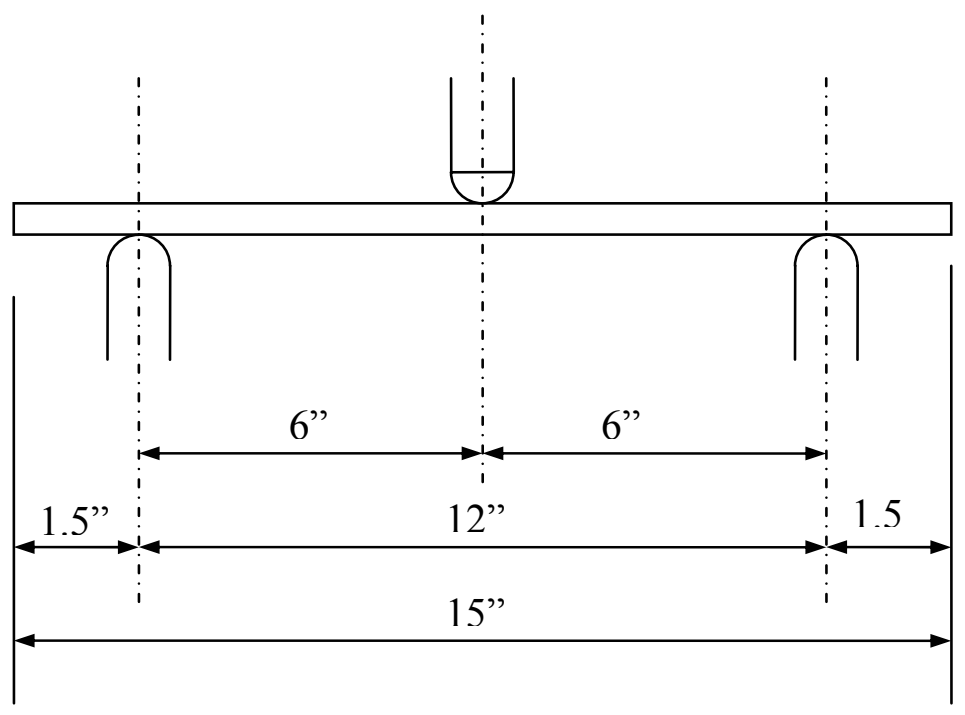

Figure 5.8 Three-point bending test setup

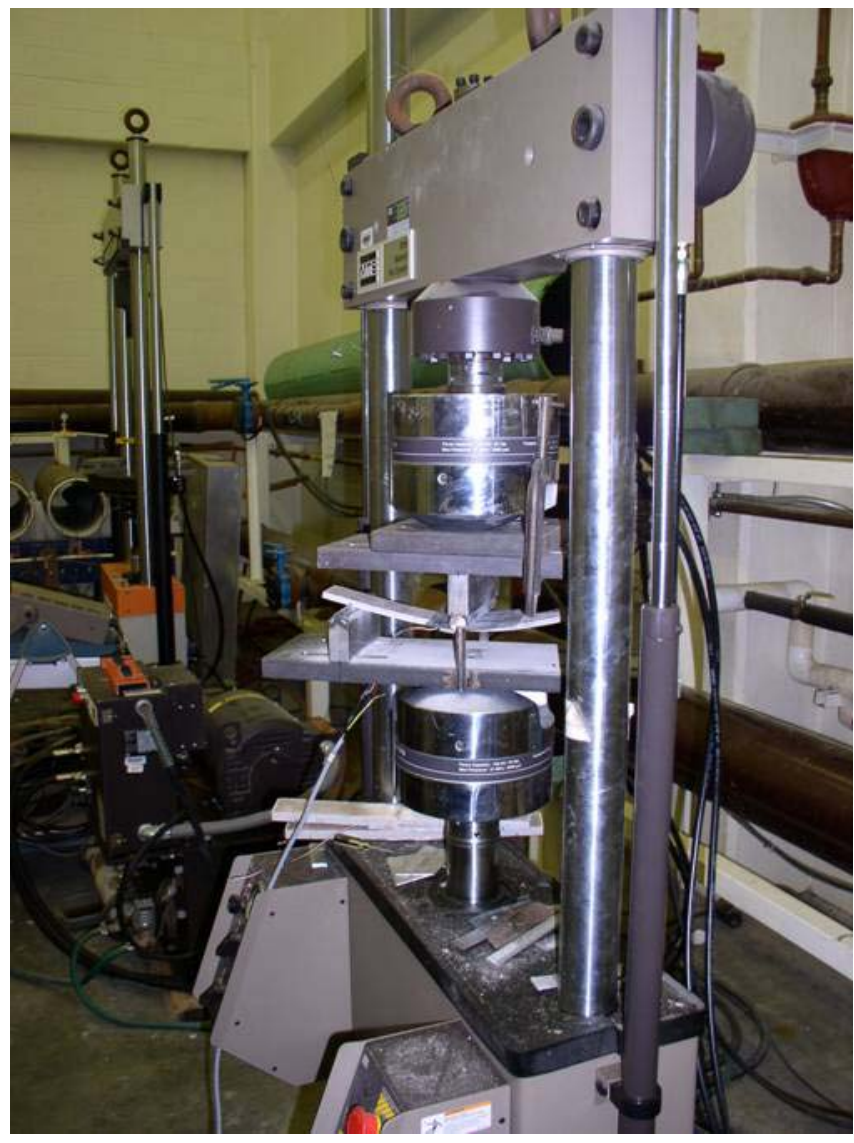

Figure 5.9 Photo of test setup 


\subsubsection{Experimental Results}

All results are given in terms of applied load vs. displacement at mid-span and applied load vs. strain at mid-span. In each case, there is good correlation in the results, and the failure mechanism observations are reproducible. Therefore, only one plot is shown for each sample. Since there is a ChSM layer at the bottom of the specimen in each configuration, which is the weakest layer, the failure always initiated from this layer and ended by crushing of top face, as shown in Fig. 5.10.

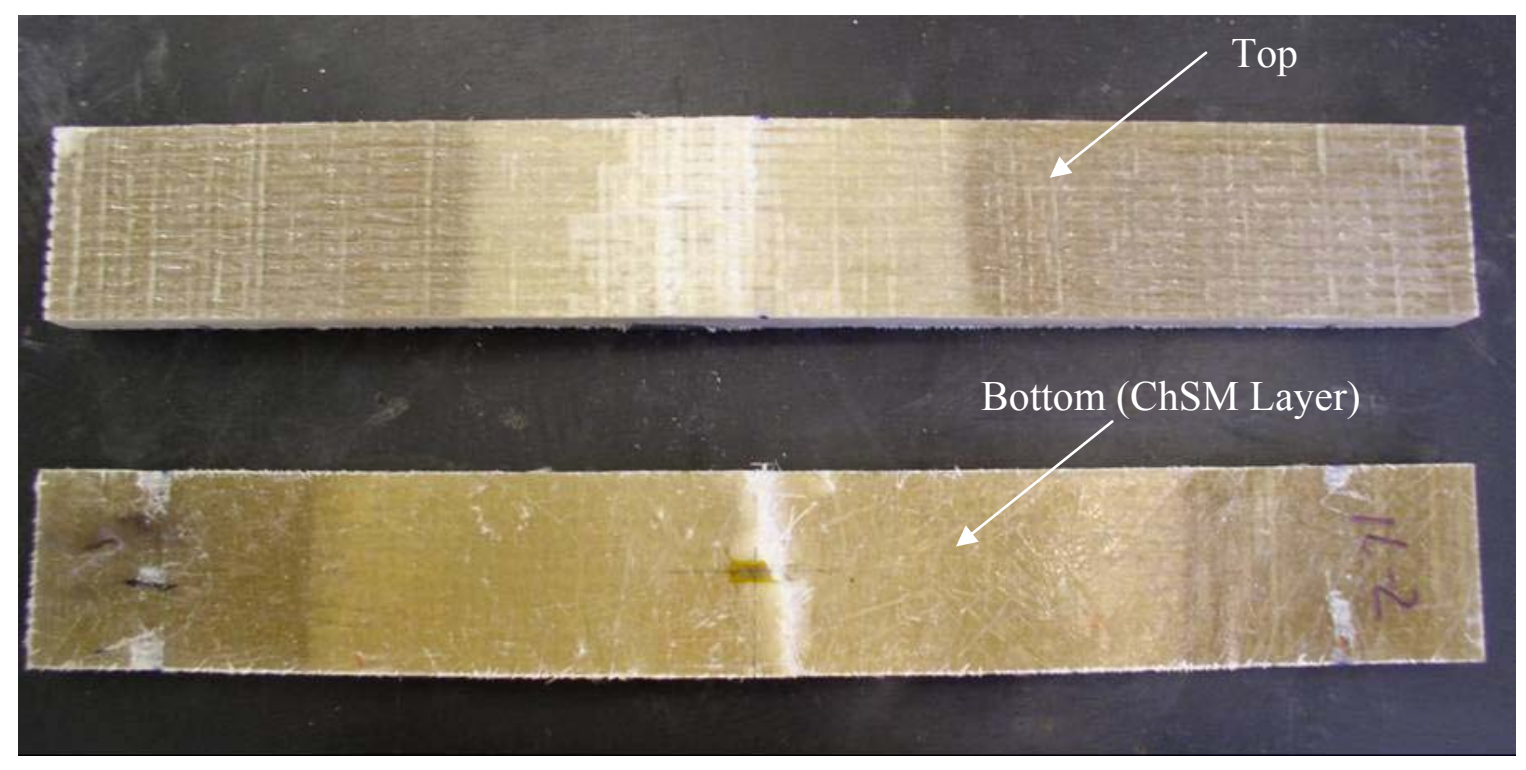

Figure 5.10 Failure mode

Table 5.8 Experimental results for bending test

\begin{tabular}{|c|c|c|c|c|}
\hline & Laminate 1L & Laminate 1T & Laminate 2 & Laminate 3 \\
\hline Failure load (lb) & 1630 & 1047 & 1609 & 1475 \\
\hline Standard deviation (lb) & 105 & 66 & 77 & 56 \\
\hline
\end{tabular}


The failure load is summarized in Table 5.8, where we can see that Laminate 1L is the strongest, followed by Laminate 2, and Laminate $1 \mathrm{~T}$ has the lowest failure load. It can be seen that although ChSM layers are introduced into laminate, the strength is not affected much. For Laminate 1L, 2, and 3, the laminates under static loadings showed nearly linear-elastic behavior up to failure, as shown in Fig. 5.11. A loud failure of ChSM layer could be observed with a sudden drop of load. Then the load was redistributed among other layers, the specimen regained some load up to a value which was slightly less than the previous peak load, and then followed by an abrupt failure. For Laminate 1T Specimens, after the ChSM layer failed, it cannot regain any load, which indicated that most of the layers failed roughly at the same time. The post-failure path was due to the residual stiffness.

From the above observation, it is concluded that all specimens assume a successive failure mode. The peak load is always associated with the failure of ChSM, which can act as the failure strength of the laminates under bending. 


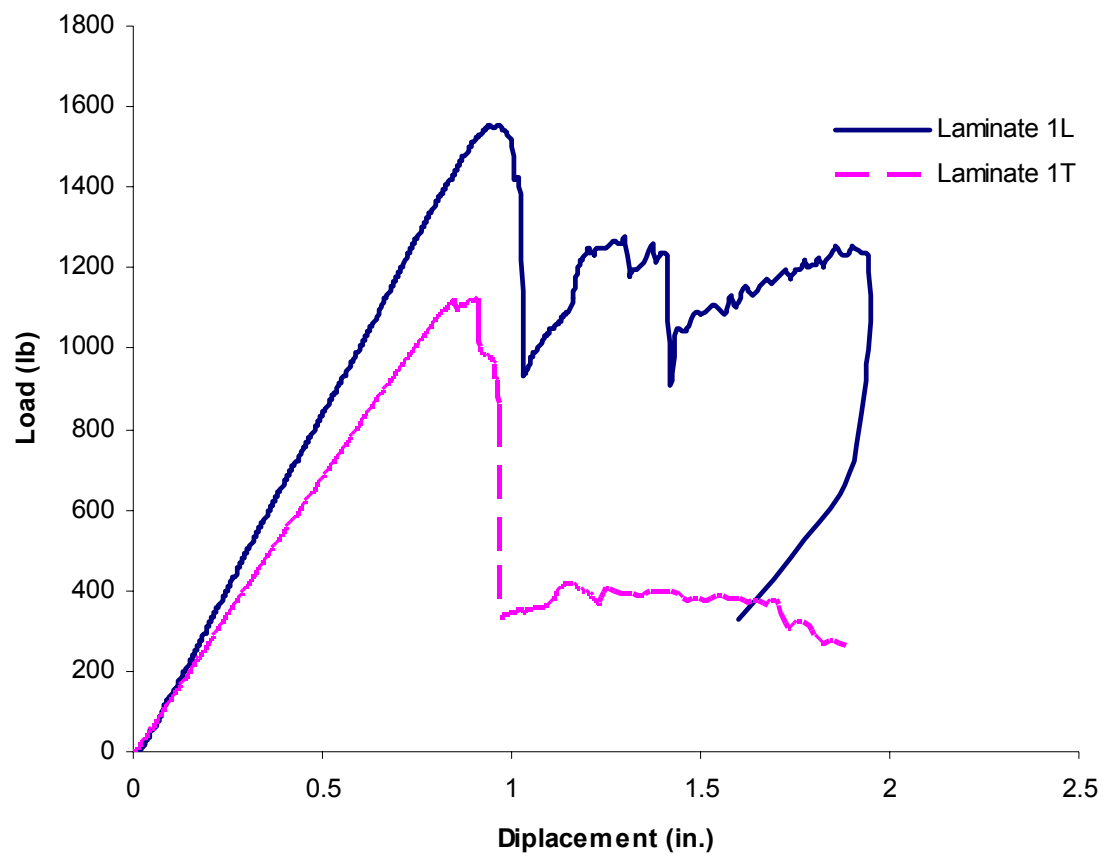

(a)

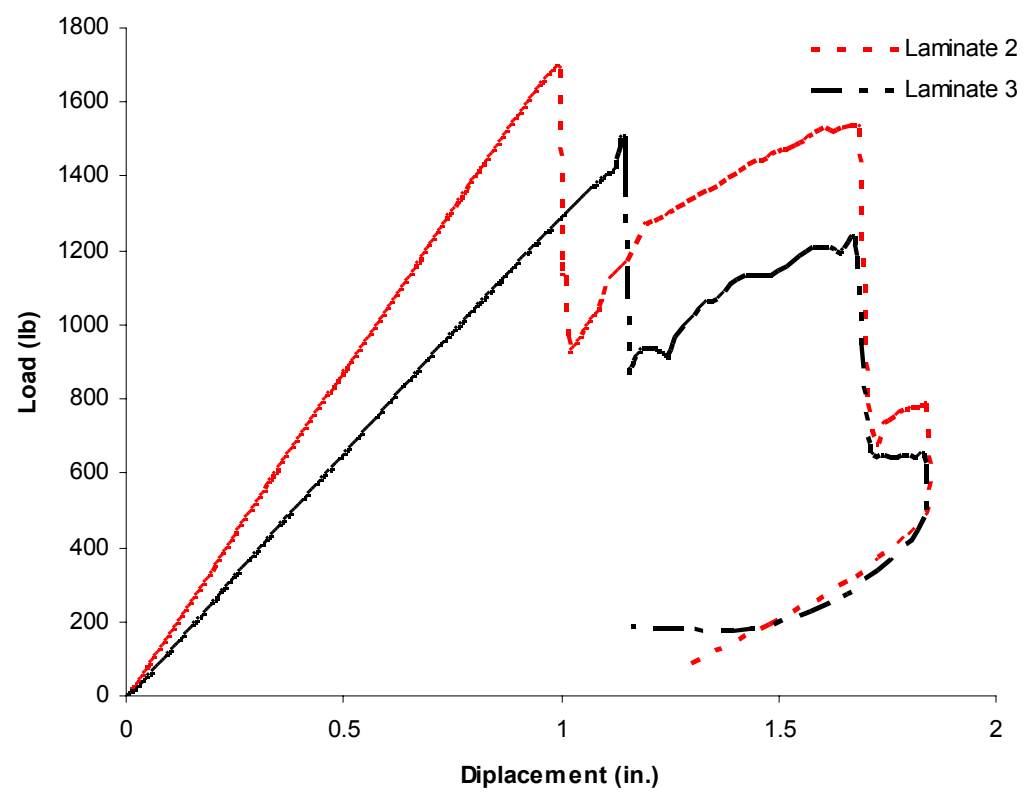

(b)

Figure 5.11 Load vs. displacement 


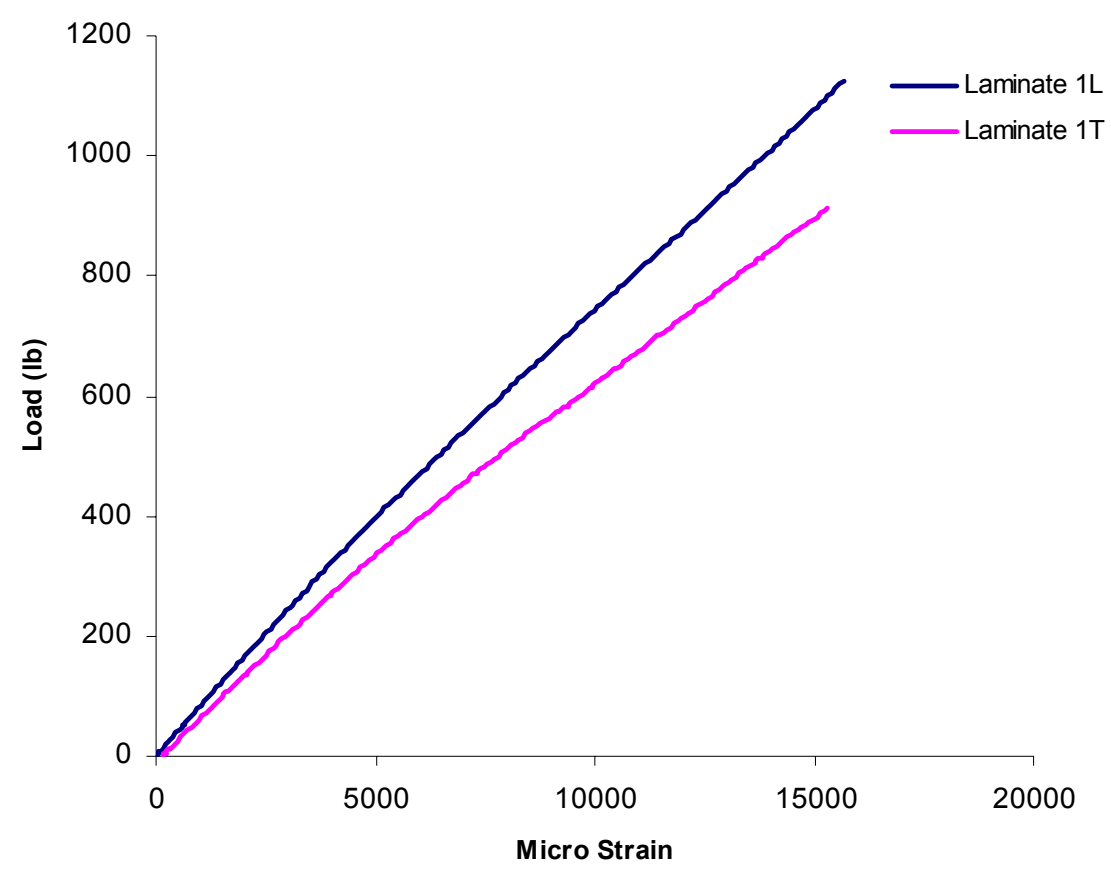

(a)

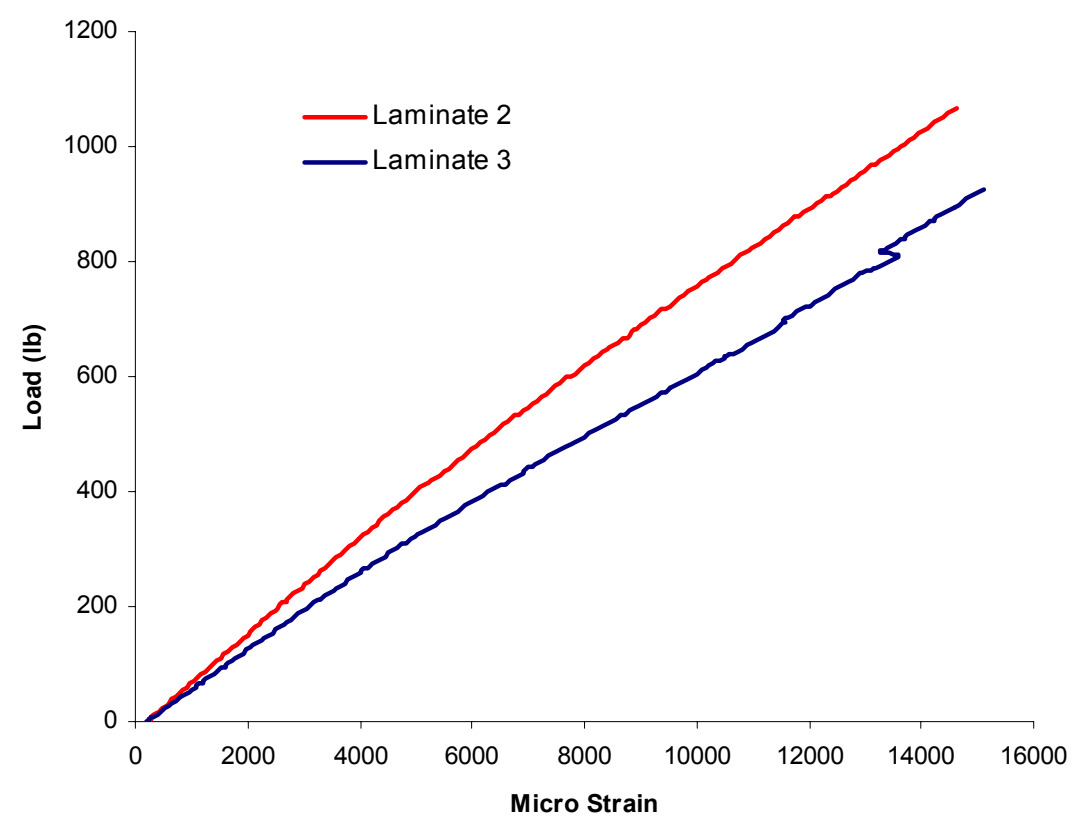

(b)

Figure 5.12 Load vs. strain 


\subsubsection{Compression Test}

Unlike other materials, such as concrete, compressive strength of composite materials is more difficult to measure due to brooming at both ends, causing premature failure, and thus, the result cannot represent the actual compressive strength. Therefore, a lot of effort has been devoted to develop appropriate compression test fixture in order to provide proper boundary conditions. ASTM specifies two methods for compression test: for specimens thinner than $1 / 8$ ", a support jig is recommended to prevent buckling; and for those thicker than $1 / 8$ ", the specimen can be tested without any support, which applies to this study. Apparently, this method cannot avoid brooming, as will be shown in this section. To solve this problem, Barbero et al. (1999) developed a novel fixture. This fixture has been used successfully to determine compressive strength of composites (Makkapati, 1994; Tomblin, 1994) and is also adopted herein (see Fig. 5.13 and Fig. 5.14). The specimens were cut into the dimension of 2 "x 1 ".

\subsubsection{Experimental Setup}

Each half of the compression fixture has two identical 5"x5" square plates, one of which has a rectangular opening in it, so that the specimen can be positioned in the grips using the side support shims which fit in these openings and are at the sides of the specimen. Once the specimen is in position, the specimen's movement is locked by using screw which moves the side support shims onto the specimen. The top grip of the fixture can slide on four guiding posts, which gives a perfect positioning and parallelism of the top grip with respect to the bottom grip. Thus, brooming of the ends is avoided by a 
restraint around the cross section of the sample on the surface of contact with the plate only. Detailed description of the fixture was given by Makkapati (1994).

All specimens were tested in a Baldwin Universal Testing machine, as shown in Fig. 5.15. The fixture uses a cylindrical loading rod, two rectangular guiding plates, which keep the ends of the specimen intact while loading. When the machine is set for loading, the loading rod pushes the upper half of the compression fixture onto the specimen, thus, the specimen is loaded. LVDTs were used to measure the movement of the loading block. Strain gages were put at the mid-height of the specimens to measure the compressive strain. 


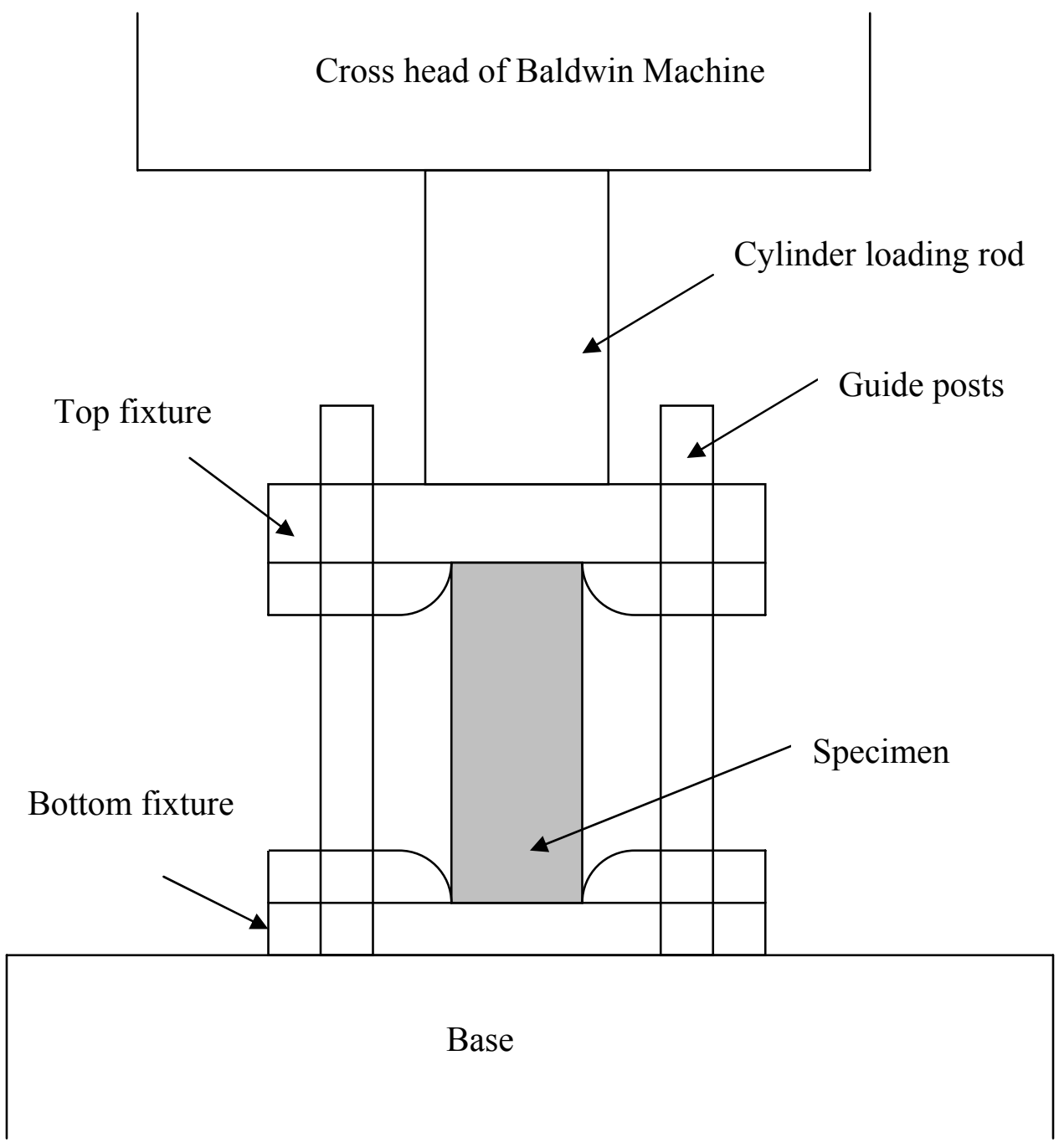

Figure 5.13 Experimental setup for compression test 

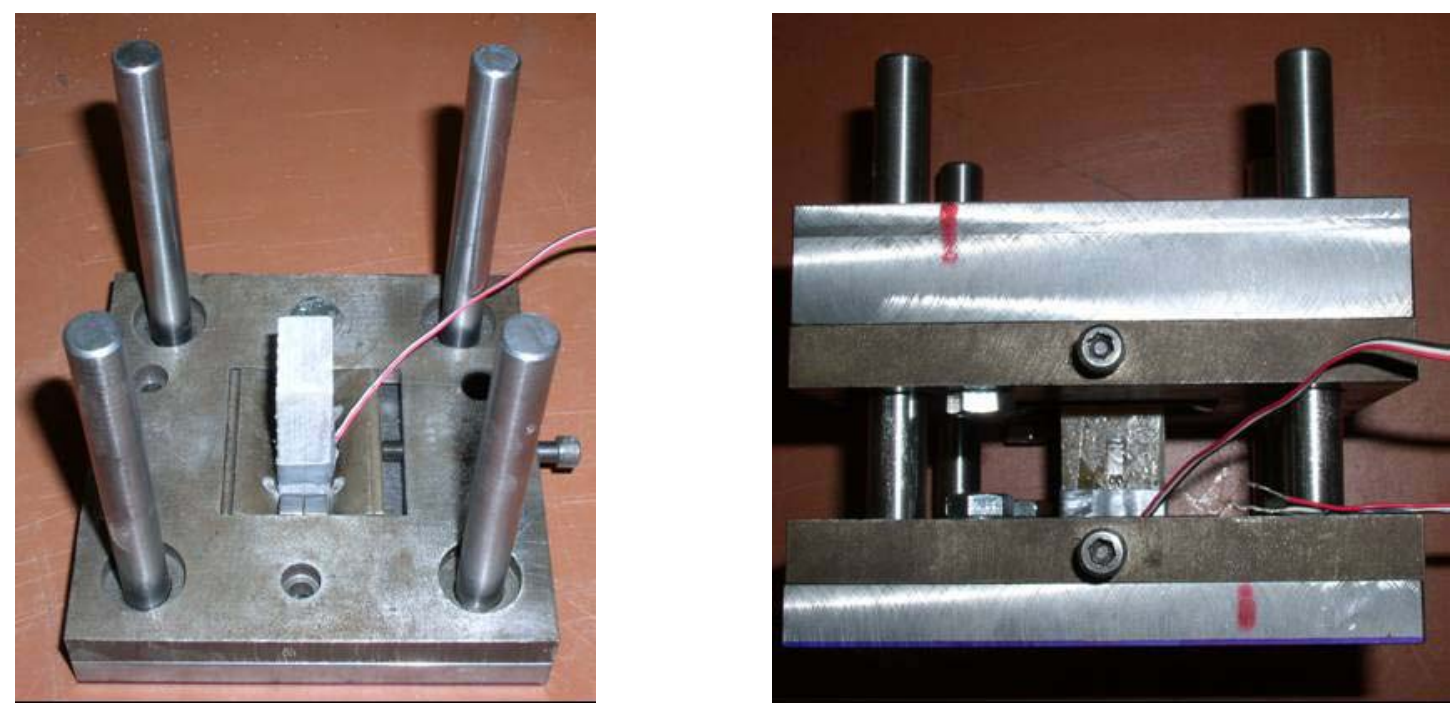

Figure 5.14 Close shot of compression fixture

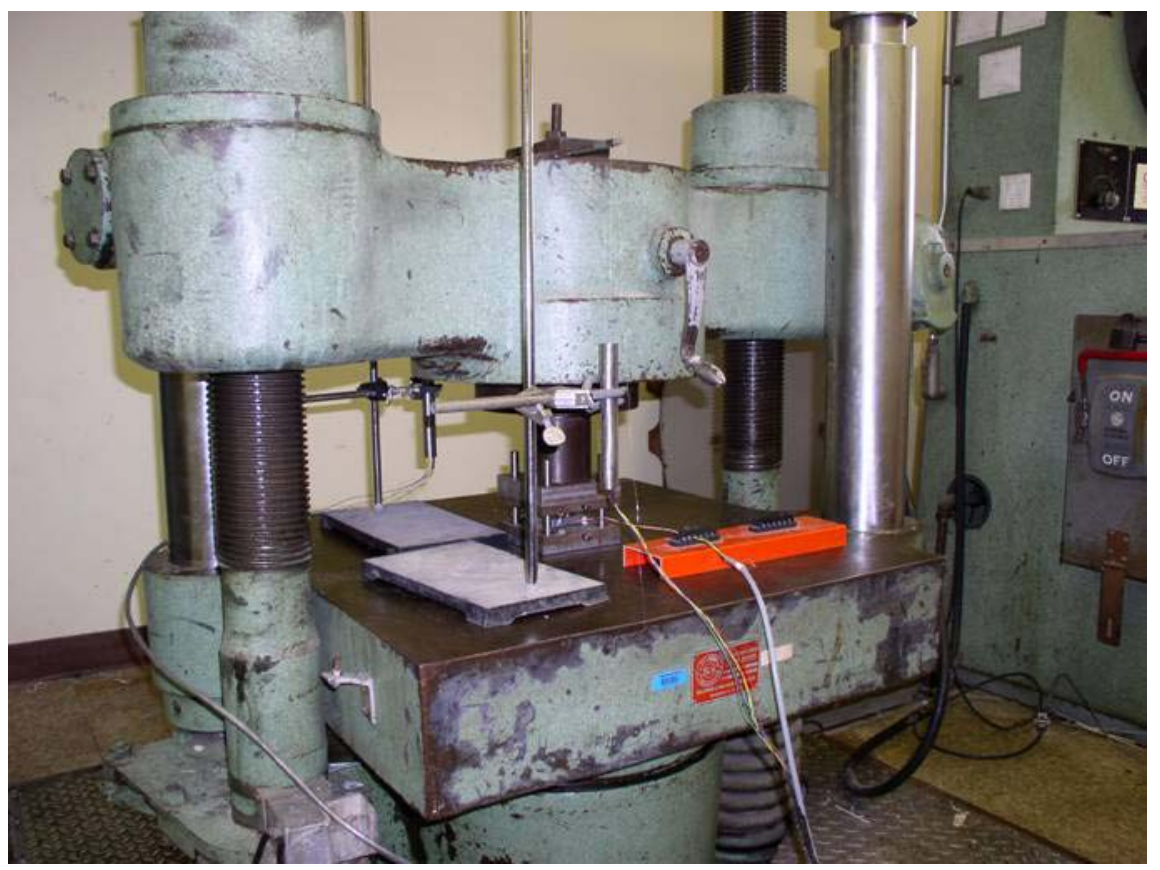

Figure 5.15 Test setup 


\subsubsection{Experimental Results}

Table 5.9 Experimental results for compression test

\begin{tabular}{|c|c|c|c|c|}
\hline & Laminate 1L & Laminate 1T & Laminate 2 & Laminate 3 \\
\hline Failure load (lb) & 26,670 & 13,931 & 23,391 & 21,903 \\
\hline Standard deviation (lb) & 1,443 & 1,213 & 1,538 & 1,114 \\
\hline
\end{tabular}

Table 5.9 gives the average failure load and standard deviation for five specimens of each type. It shows that the results obtained from the experimental program are fairly consistent and the standard deviation is within $10 \%$ of the strength of the specimens. As expected, Laminate 1L and 1T correspond to the highest and lowest failure load, respectively, with Laminate 2 and 3 in between. This corroborates the conclusions drawn from Section 5.2, that the strength is not affected much when ChSM is introduced into face laminates.

During the test, the specimen was intact until the maximum load was reached. It failed with a loud sound and a sudden drop of the load. Fig. 5.16 and Fig. 5.17 plot load vs. displacement and load vs. strain at mid-span, respectively, showing a typical linear behaviour up to failure except for Laminate 3, where some nonlinear behaviour can be observed. A typical failure mode is shown in Fig. 5.18, indicating the compressive failure for a laminate achieved.

As a matter of interest, three specimens were tested according to ASTM standards, i.e., without the end support offered by the compression fixture. The comparison between these two failure modes is shown in Fig. 5.19, where we can see an 
apparent end brooming of the unconstrained specimen and a premature failure by the laminate separation. As a result, the specimen failed at a much lower load of 19,575 $\mathrm{lb}$.

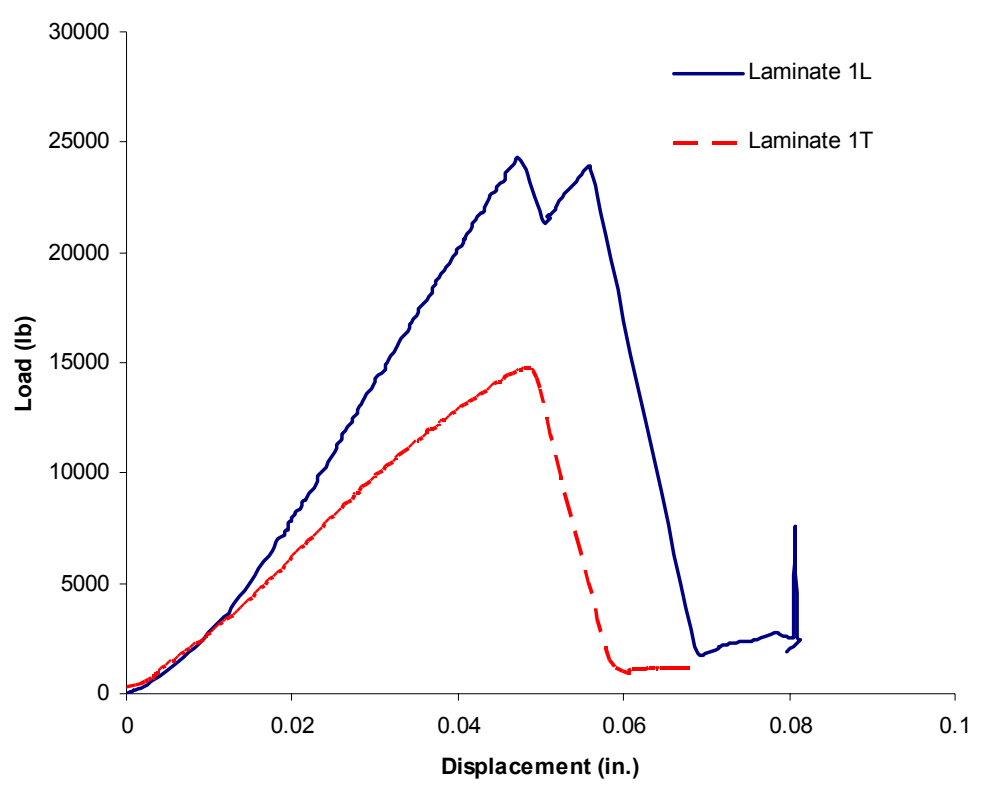

(a)

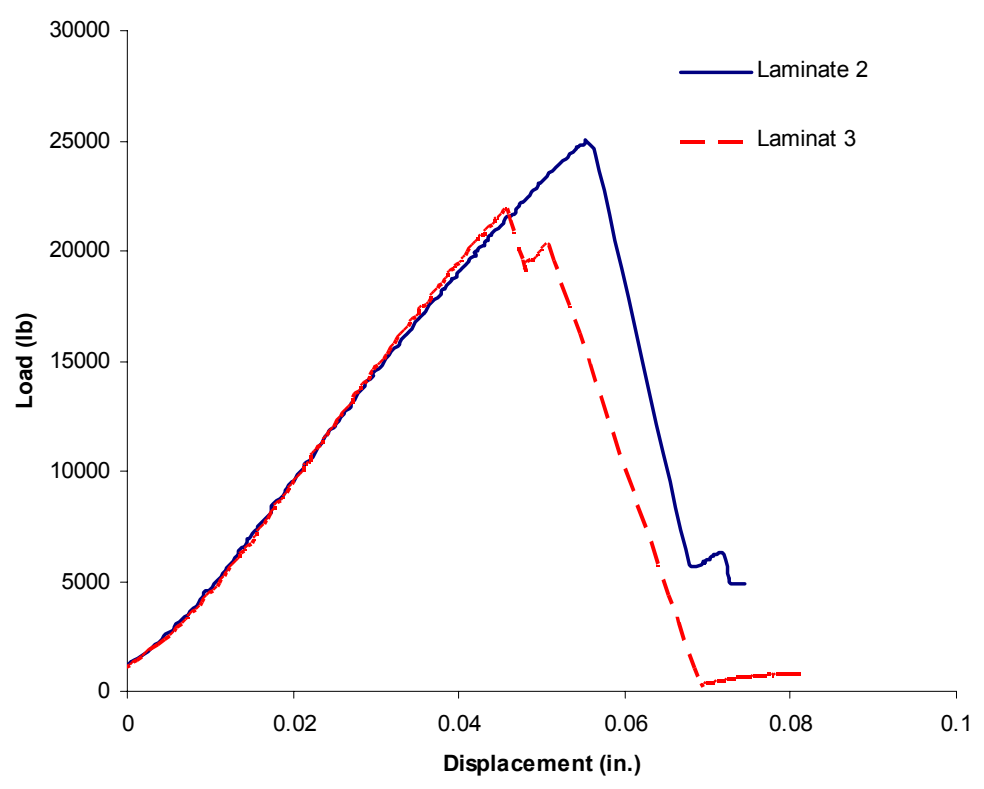

(b)

Figure 5.16 Load-displacement curve 


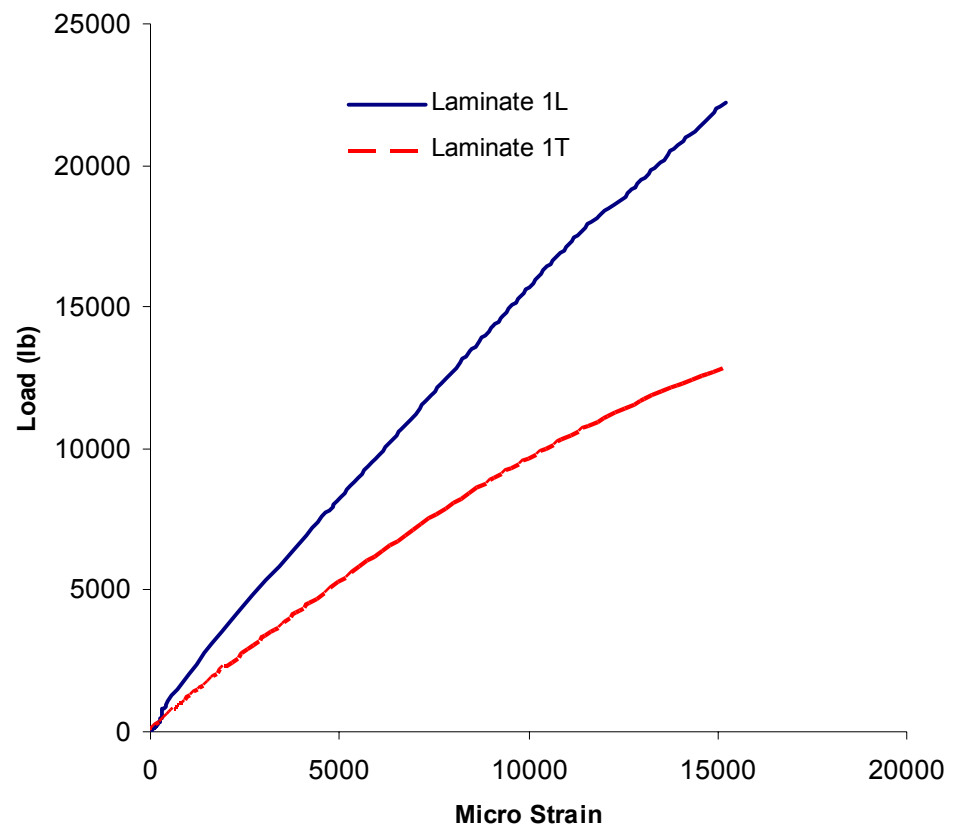

(a)

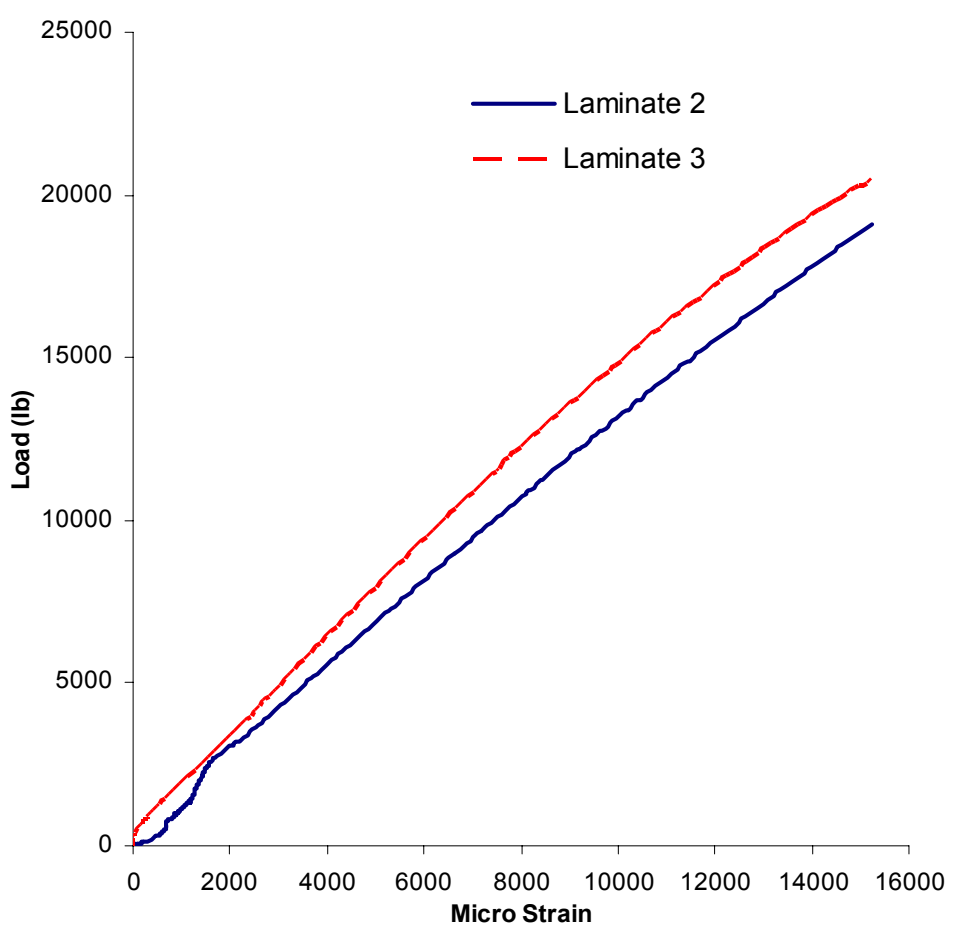

(b)

Figure 5.17 Load-strain curve 


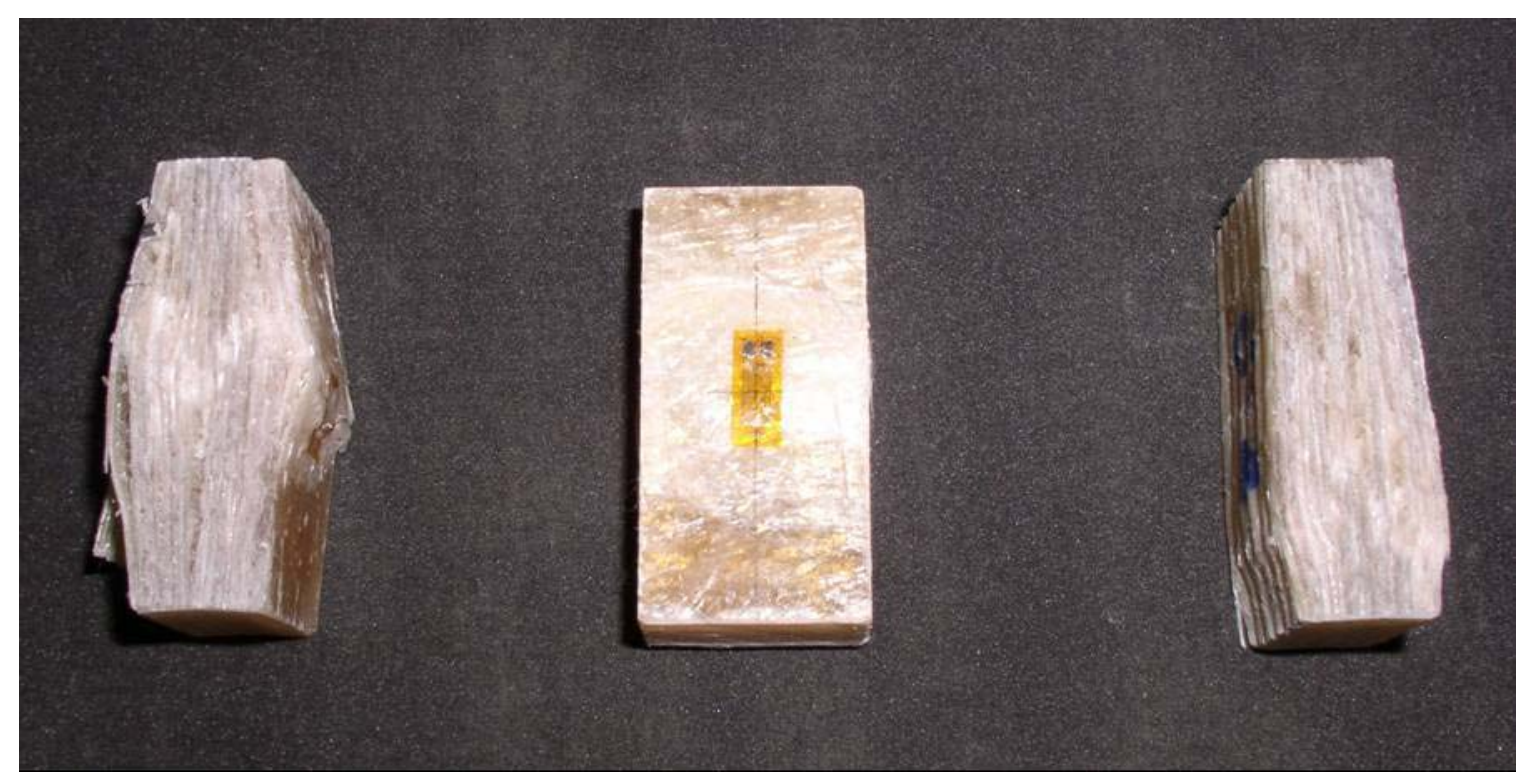

Figure 5.18 Failed specimen

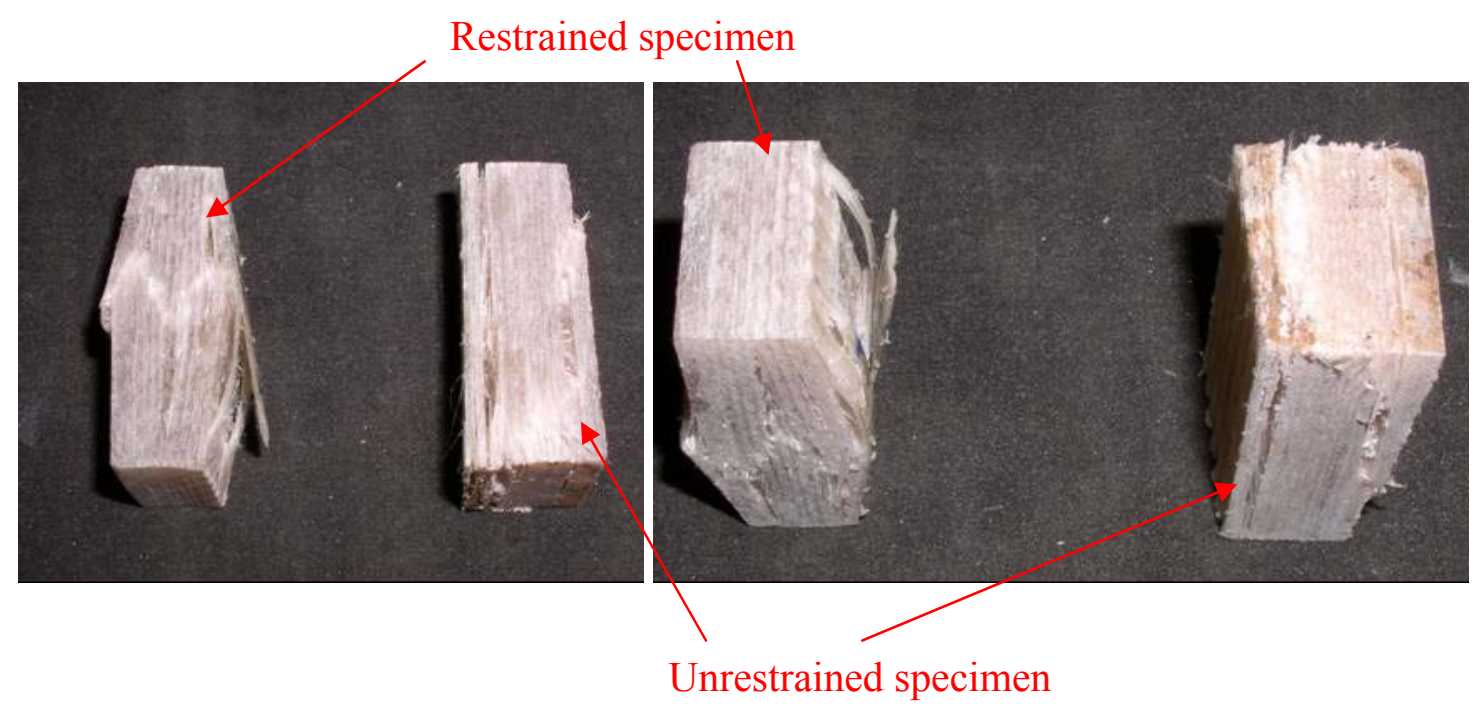

(a) Side view

(b) Top view

Figure 5.19 Failure mode comparison 


\subsection{Correlation between FE and Experimental Results}

Table 5.10 Properties of constituent materials

\begin{tabular}{|c|c|c|c|c|}
\hline Material & $\mathbf{E}\left(\mathbf{x 1 0 ^ { 6 }} \mathbf{p s i}\right)$ & $\mathbf{G}\left(\mathbf{x 1 0}^{\mathbf{6}} \mathbf{p s i}\right)$ & $\boldsymbol{v}$ & $\boldsymbol{\rho}, \mathbf{o z} / \mathbf{i n}^{\mathbf{3}}$ \\
\hline E-Glass Fiber & 10.5 & 4.3 & 0.22 & 1.480 \\
\hline Polyester (Isophthalic) Resin & 0.530 & 0.192 & 0.38 & 0.636 \\
\hline
\end{tabular}

Table 5.11 Layer properties of face laminates

\begin{tabular}{|c|c|c|c|c|}
\hline Ply name & Ply type & $\begin{array}{c}\text { Nominal weight } \\
\left(\mathrm{oz} / \mathbf{f t}^{2}\right)\end{array}$ & $\begin{array}{l}\text { Thickness } \\
\text { (in.) }\end{array}$ & $\mathbf{V}_{\mathbf{f}}$ \\
\hline CDM 3208 & $\begin{array}{c}0^{\circ} \\
90^{\circ} \\
\mathrm{ChSM}\end{array}$ & $\begin{array}{l}1.74 \\
1.97 \\
0.84\end{array}$ & $\begin{array}{c}0.0193 \\
0.0217 \\
0.01\end{array}$ & $\begin{array}{l}0.4241 \\
0.4251 \\
0.3962\end{array}$ \\
\hline CM 1708 & $\begin{array}{c}0^{\circ} \\
\mathrm{ChSM}\end{array}$ & $\begin{array}{l}1.71 \\
0.84\end{array}$ & $\begin{array}{c}0.027 \\
0.01\end{array}$ & $\begin{array}{l}0.2947 \\
0.3962\end{array}$ \\
\hline Bonding layer & ChSM & 3 & 0.075 & 0.1877 \\
\hline
\end{tabular}


Table 5.12 Material properties

\begin{tabular}{|c|c|c|c|c|c|c|}
\hline Type & $\begin{array}{c}\mathbf{E}_{1} \\
\left(\mathrm{x} 10^{6} \mathrm{psi}\right)\end{array}$ & $\begin{array}{c}E_{2} \\
\left(\times 10^{6} \mathrm{psi}\right)\end{array}$ & $\begin{array}{c}\mathbf{G}_{12} \\
\left(\times 10^{6} \mathrm{psi}\right)\end{array}$ & $\begin{array}{c}\mathbf{G}_{23} \\
\left(\times 10^{6} \mathrm{psi}\right)\end{array}$ & $v_{12}$ & $v_{23}$ \\
\hline $\mathrm{CDM} 32080^{\circ} / 90^{\circ}$ & 5.206 & 1.610 & 0.481 & 0.440 & 0.305 & 0.599 \\
\hline CDM 3208 CSM & 2.527 & 2.527 & 0.899 & 0.899 & 0.406 & 0.406 \\
\hline $\mathrm{CM} 17080^{\circ}$ & 3.468 & 1.072 & 0.334 & 0.313 & 0.333 & 0.599 \\
\hline CM 1708 CSM & 2.527 & 2.527 & 0.899 & 0.899 & 0.406 & 0.406 \\
\hline Bonding layer & 1.424 & 1.424 & 0.509 & 0.509 & 0.397 & 0.397 \\
\hline
\end{tabular}

Table 5.13 Strength parameters (psi)

\begin{tabular}{|c|c|c|c|c|c|c|}
\hline Type & $\mathbf{X}_{\mathbf{T}}$ & $\mathbf{X}_{\mathbf{C}}$ & $\mathbf{Y}_{\mathbf{T}}$ & $\mathbf{Y}_{\mathbf{C}}$ & $\mathbf{S}_{\mathbf{1 2}}$ & $\mathbf{S}_{\mathbf{2 3}}$ \\
\hline $\mathrm{CM} 32050^{\circ} / 90^{\circ}$ & 226800 & 80693 & 7350 & 9817 & 6362 & 6362 \\
\hline $\mathrm{CM} 3205 \mathrm{ChSM}$ & 40432 & 40432 & 40432 & 40432 & 20216 & 20216 \\
\hline $\mathrm{UM} \mathrm{1810} \mathrm{0^{ \circ }}$ & 165300 & 49650 & 7284 & 9947 & 6447 & 6447 \\
\hline UM 1810 ChSM & 40432 & 40432 & 40432 & 40432 & 20216 & 20216 \\
\hline Bond layer ChSM & 22784 & 22784 & 22784 & 22784 & 11392 & 11392 \\
\hline
\end{tabular}

As the manufacturing company has adopted new laminas into their facesheet configurations, the materials properties should be updated. The properties of constituent materials are listed in Table 5.10. The stiffness of properties of composite materials depends on the relative volume of fiber $\left(V_{f}\right)$ and matrix used. For a fiber mat with nominal weight $(\omega), V_{f}$ can be determined from 


$$
V_{f}=\frac{\omega}{\rho \cdot t}
$$

where $t$ is the thickness of the layer and $\rho$ is the density of E-glass fibers. For the face laminates given, the fiber volume fraction for each layer is computed from Eq. (5.6) and shown in Table 5.11. The stiffness of each ply can be predicted from micromechanics models (Luciano and Barbero, 1994) and listed in Table 5.12. The strength parameters shown in Table 5.13 are calculated using CADEC (Barbero, 1999).

\subsubsection{Three-Point Bending}

Table 5.14 Comparison of failure load for three-point bending test

\begin{tabular}{|c|c|c|c|c|}
\hline & Laminate 1L & Laminate 1T & Laminate 2 & Laminate 3 \\
\hline Test result (lb) & 1631 & 1047 & 1609 & 1475 \\
\hline FE result (lb) & 1751 & 1117 & 1721 & 1360 \\
\hline Difference (\%) & 7.4 & 6.7 & 6.9 & 7.8 \\
\hline
\end{tabular}




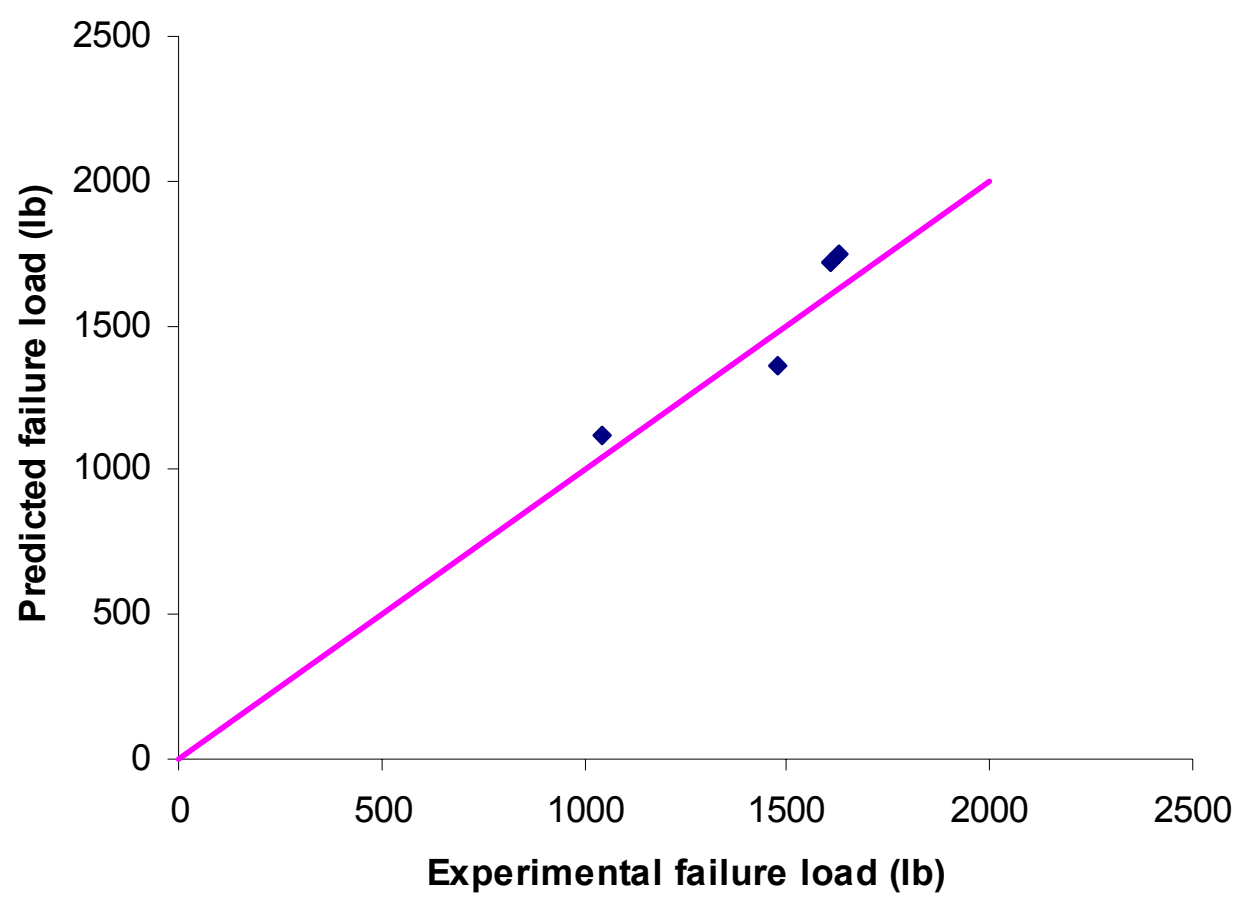

Figure 5.20 Comparison of failure load

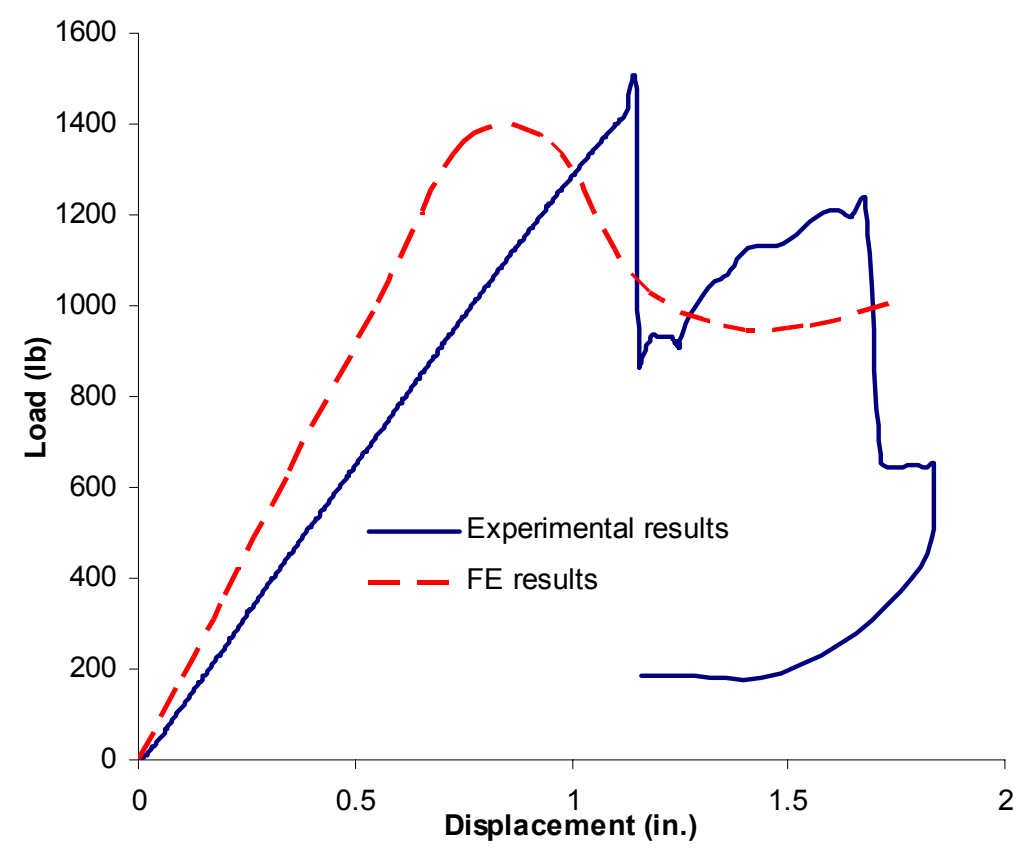

Figure 5.21 Load-displacement paths for Laminate 3 


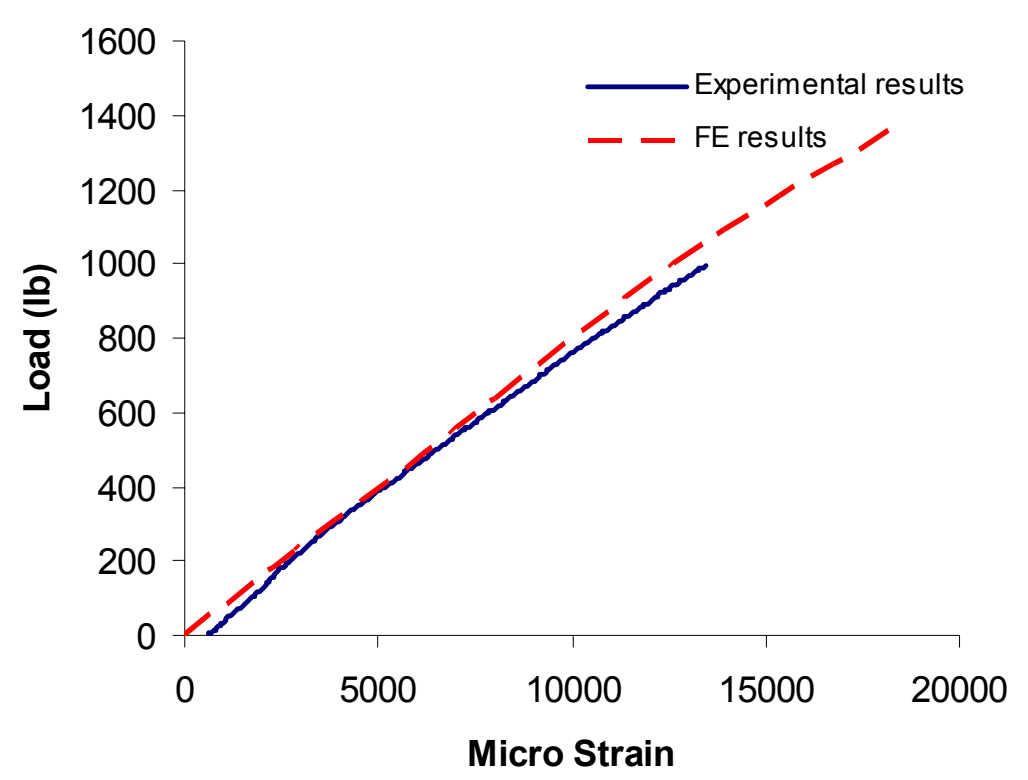

Figure 5.22 Load-strain paths for Laminate 3

Using the progressive failure model developed in this chapter, predictions of the compressive strength may be determined, as shown in Table 5.14, from which we can see that predictions from the FE model closely approximates the experimentally obtained results, with a maximum difference of $7.7 \%$. Fig. 5.20 compares maximum load from FE prediction and test results, illustrating a good correlation.

The predicted vs. experimental load-displacement and load-strain curves for selected Laminate 3 are shown in Fig. 5.21 and Fig. 5.22, where good agreement can be observed, although some discrepancies for post failure paths can be noticed. It is noted that load-strain curve correlates better than load-displacement curve, due to the fact that the displacement recorded is the movement of the loading head, and therefore, it cannot represent the actual deflection of the specimen. The diagrams for other types of laminate are listed in Fig. 5.28 through Fig. 5.33 at the end of this chapter. 


\subsubsection{Compression Test}

Following the same approach as described for three-point bending, predictions of the compressive strength are listed in Table 5.15 together with those from tests. Once again, predictions from the FE model closely approximate the experimentally results obtained except Laminate $1 \mathrm{~T}$, with a percentage difference of $24.0 \%$. Two factors may contribute to this difference: 1) some nonlinearity was observed during the test; and 2) the compressive strength along the transverse direction is very difficult to predict for a lamina. Figure 5.23 compares maximum load from FE prediction and test results, illustrating a good correlation.

The predicted vs. experimental load-displacement and load-strain diagrams for selected Laminate 3 are shown in Fig. 5.24 and Fig. 5.25, where good agreement can be observed. The diagrams for other types of laminate are listed in Fig. 5.34 through Fig. 5.39 at the end of this chapter.

Table 5.15 Comparison of failure load for compression test

\begin{tabular}{|c|c|c|c|c|}
\hline & Laminate 1L & Laminate 1T & Laminate 2 & Laminate 3 \\
\hline Test result (lb) & 26670 & 13931 & 23391 & 21903 \\
\hline FE result (lb) & 26168 & 17274 & 21212 & 19722 \\
\hline Difference (\%) & 1.9 & 24.0 & 9.3 & 9.9 \\
\hline
\end{tabular}




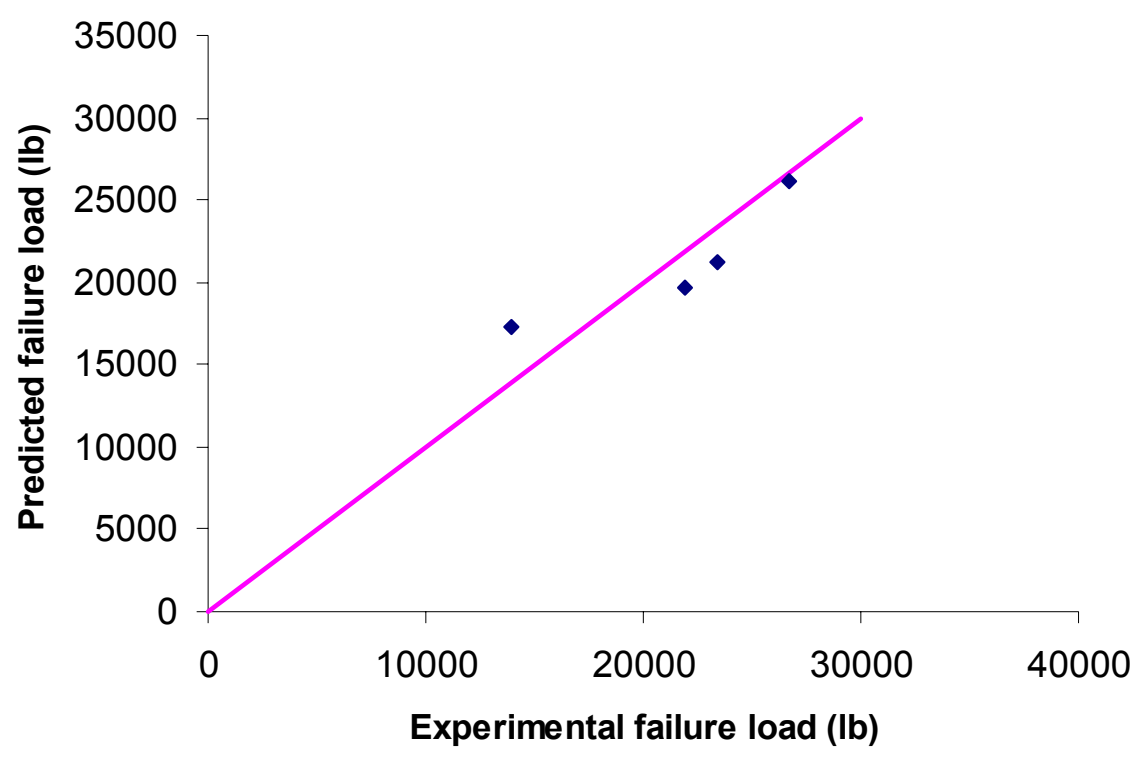

Figure 5.23 Comparison of failure load

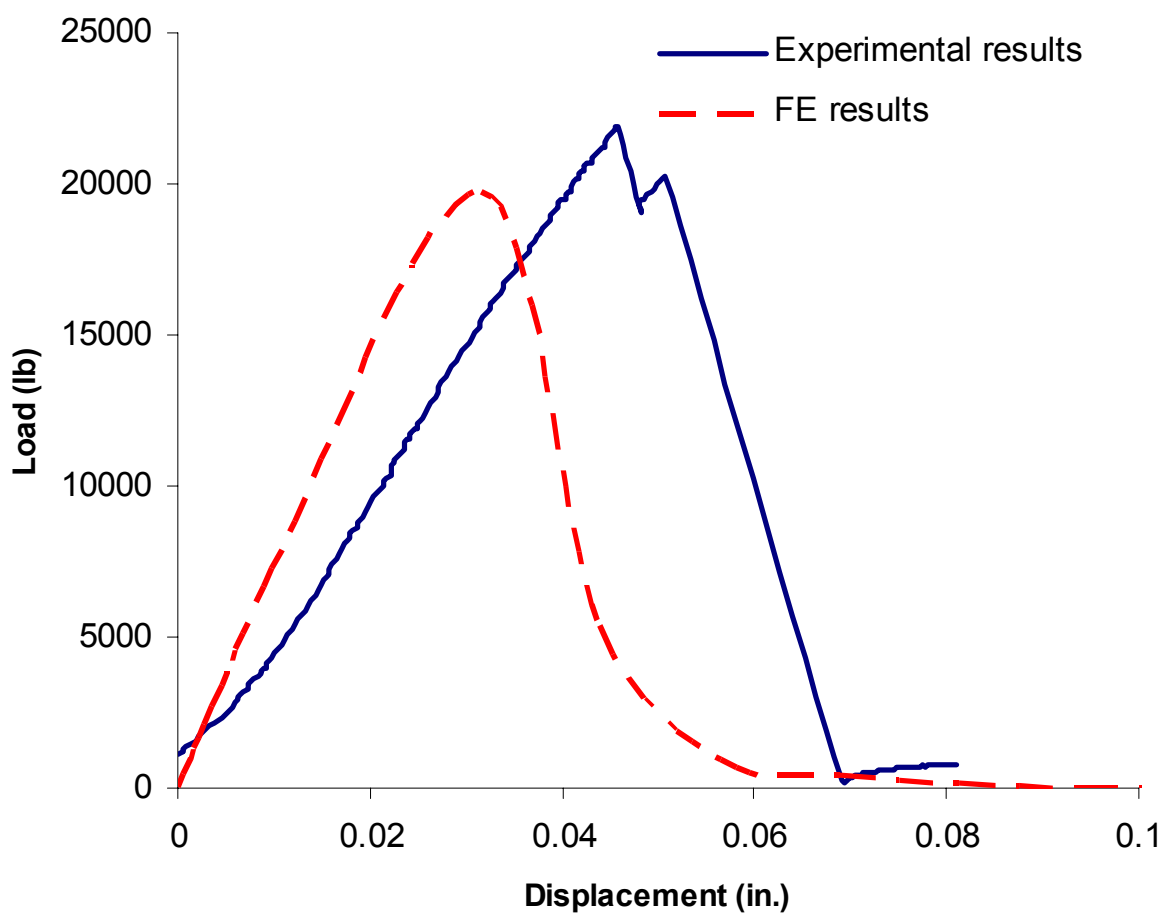

Figure 5.24 Load-displacement paths for Laminate 3 


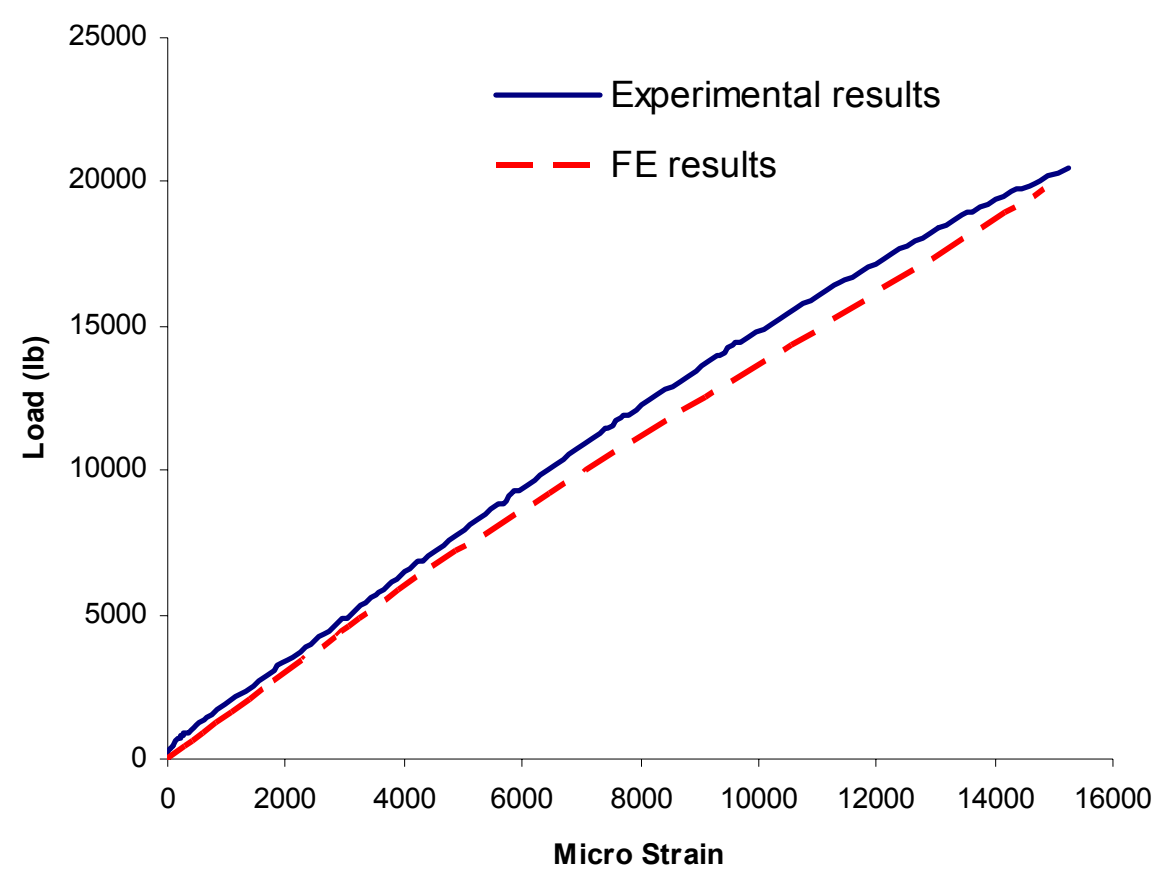

Figure 5.25 Load-strain paths for Laminate 3

\subsection{Discussions}

To illustrate the change of stiffness for each laminate shown in Section 4.5, using the updated material properties, the same static analysis, as described in Section 4.4, is carried out for a patch load of 20 kips acting at the center of an 8'x8' sandwich panel with 8 " thick core. The results are shown in Table 5.16 and the normalized deflection based on Laminate 1L is shown in Fig. 5.26. Using the compression test data, the normalized compressive strength based on Laminate 1L is shown in Fig. 5.27.

From Fig. 5.26 and Fig. 5.27, we can observe that for Laminate 2, the strength is $13 \%$ lower and the deflection is $10 \%$ higher; and for Laminate 3 , the strength is $18 \%$ lower and the deflection is $4 \%$ higher than Laminate $1 \mathrm{~L}$. However, the deflection for Laminate 2, which is $\mathrm{L} / 960$, where $\mathrm{L}$ is the span of the deck, is still in the acceptable 
range. If other factors are also considered, such as the cost and manufacturing process, Laminate 2 is recommended for the facesheet of sandwich bridge deck panels. Manufactures can make their own decisions based on the stiffness and strength comparisons provided in Fig. 5.26 and 5.27.

Table 5.16 Deflection under patch load for 8' $x 8^{\prime}$ plate

\begin{tabular}{|c|c|c|c|c|}
\hline Laminate & $1 \mathrm{~L}$ & $1 \mathrm{~T}$ & 2 & 3 \\
\hline Deflection & $0.0909 ”$ & $0.1174 ”$ & $0.1000 \%$ & $0.0942 \%$ \\
\hline
\end{tabular}

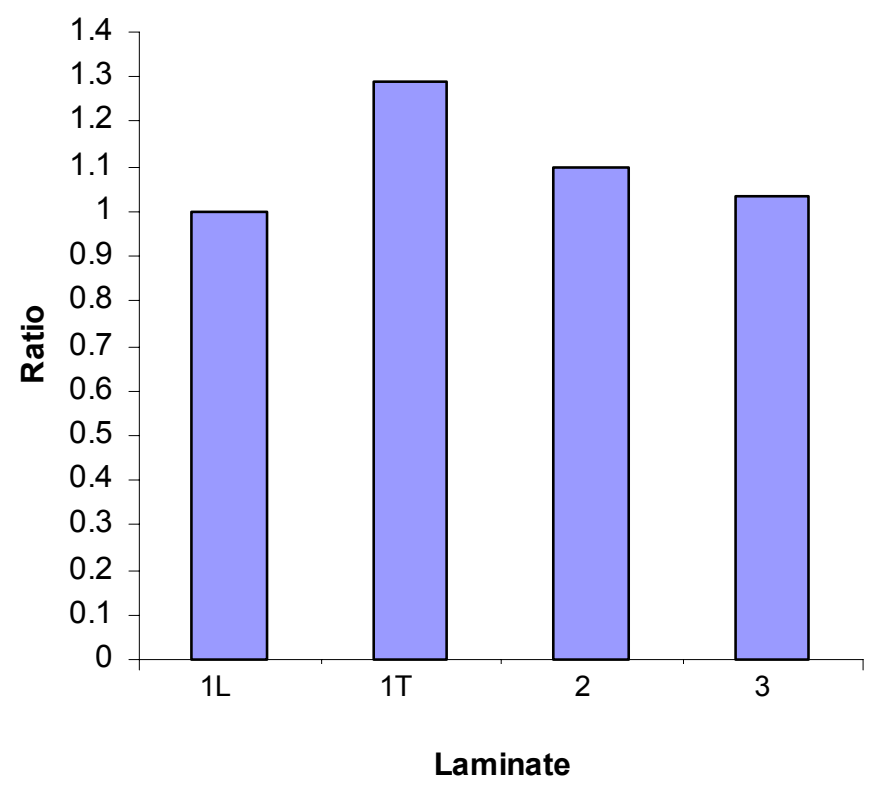

Figure 5.26 Normalized deflection under patch load 


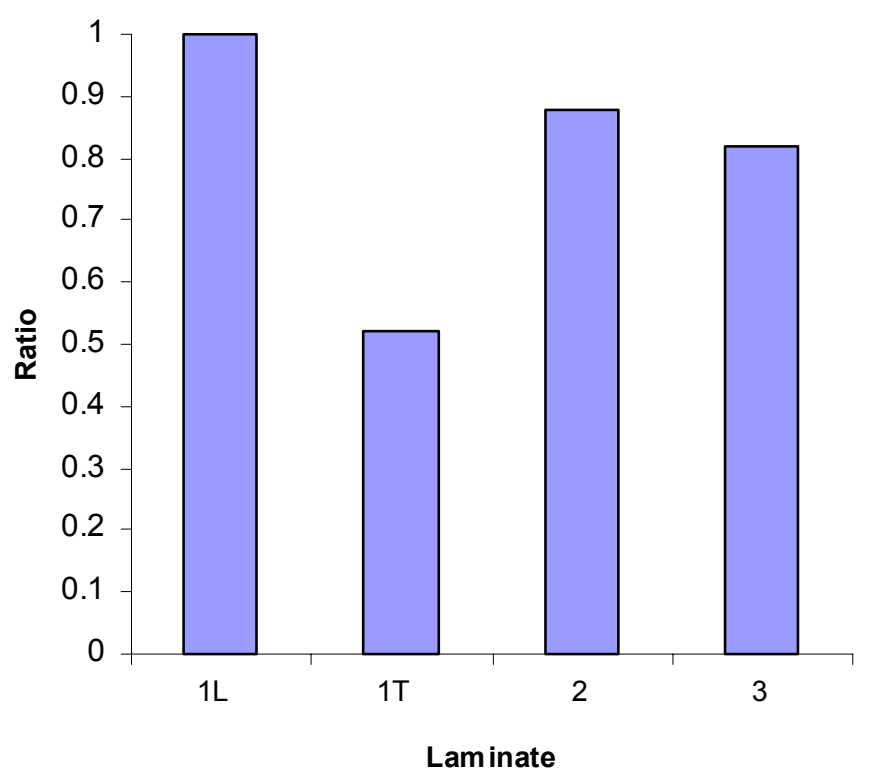

Figure 5.27 Normalized strength

\subsection{Conclusions}

A progressive failure model is developed using FE method to predict the behavior of laminated composite plates up to failure. A parametric study is carried out on strength properties of the facesheet for a sandwich panel using this model. Compressive and bending tests are carried out on selected configurations. From this study, the following conclusions can be drawn:

- The progressive failure model developed in this chapter can be successfully used to predict the behaviour of laminated composite plates, as proved from the correlation between FE results and existing experimental data. It is much more efficient compared to 3-D model and offers great potential for a parametric study; 
- Interlaminar shear strength should be carefully considered when the delamination occurs prior to other failure modes;

- From the parametric study, it is shown that, for a composite laminate, the compressive strength is lower than the tensile strength. If it is used for the facesheet of a sandwich bridge deck panel, as the top and bottom facesheet are in compression or tension with equal magnitude, the compressive strength of the facesheet is more critical and controls the design;

- If Chopped Strand Mat layer is introduced into the facesheet, the strength is not affected much, while the stiffness is reduced, resulting in a larger deflection under the same loading condition;

- Three-point bending tests were conducted where a progressive failure mode was observed. Compression tests were carried out on a novel fixture, where the endbrooming is avoided and the true compressive strength is obtained. The results from the bending and compression tests on selected configurations further validate the progressive failure model derived;

- The existing facesheet in industry is too conservative. Based on the results from this study, an optimized Laminate 2 is recommended for future applications. 


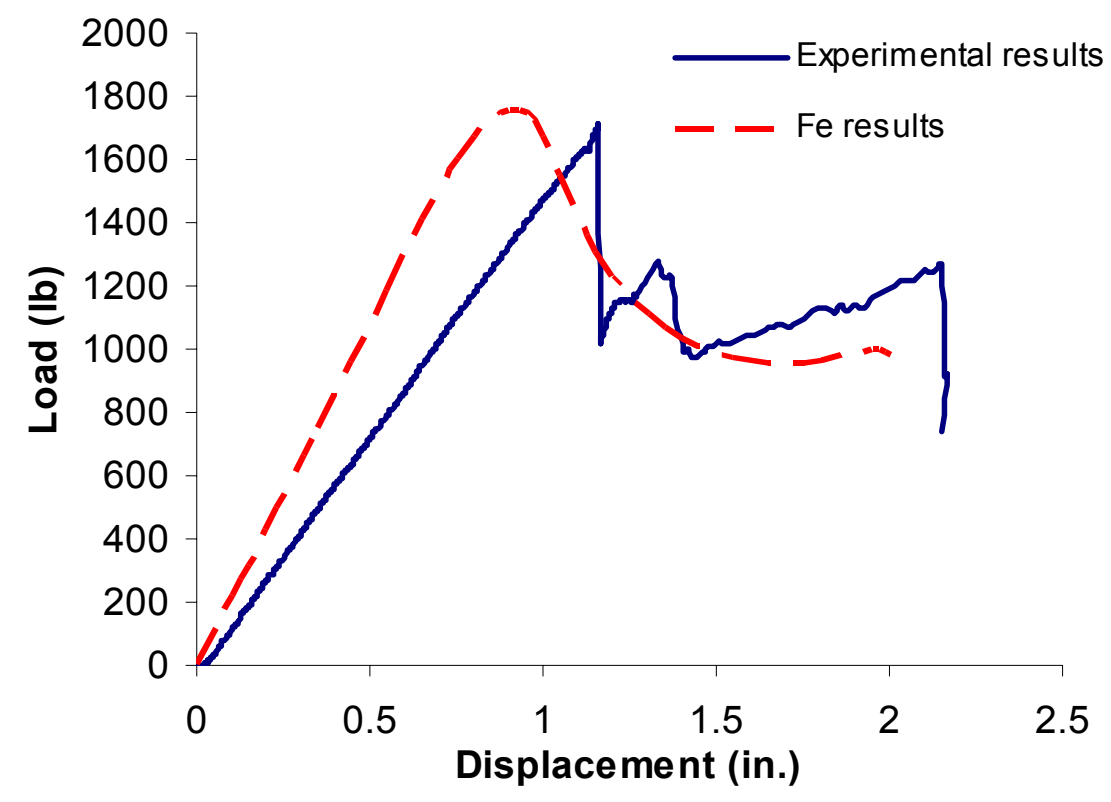

Figure 5.28 Load-displacement paths for Laminate 1L under bending

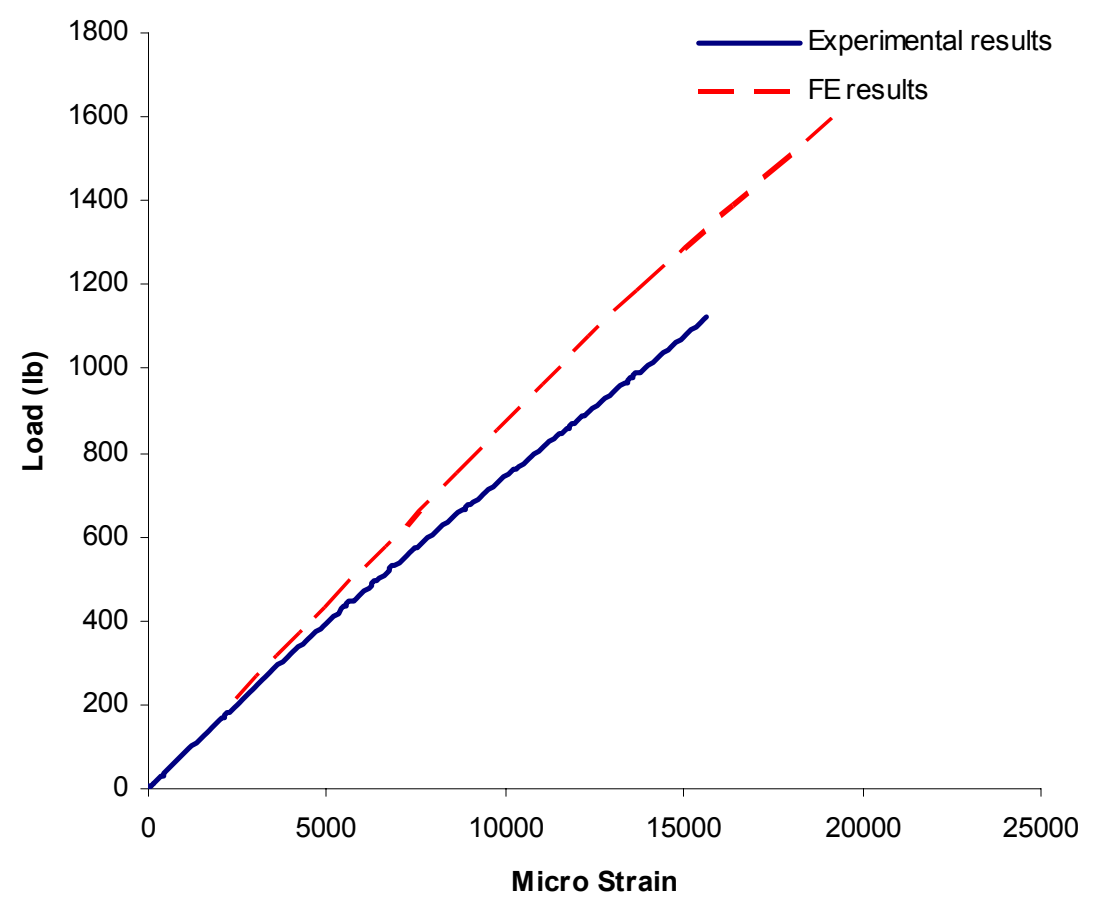

Figure 5.29 Load-strain paths for Laminate 1L under bending 


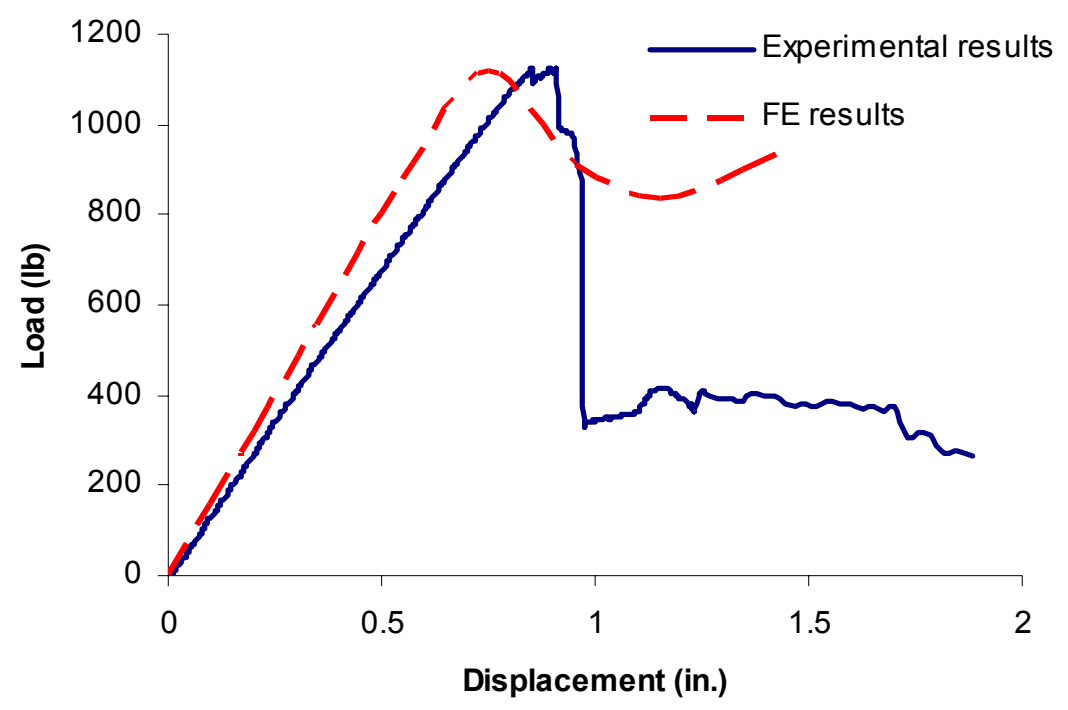

Figure 5.30 Load-displacement paths for Laminate 1T under bending

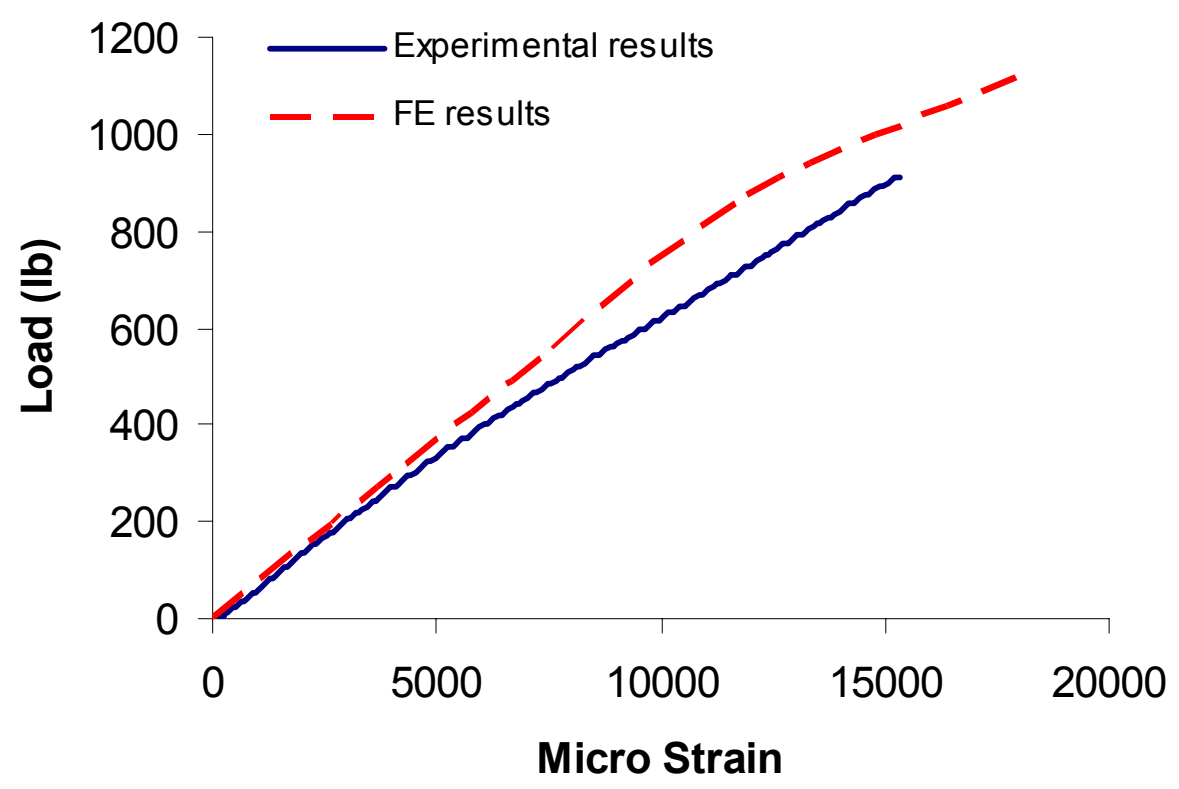

Figure 5.31 Load-strain paths for Laminate 1T under bending 


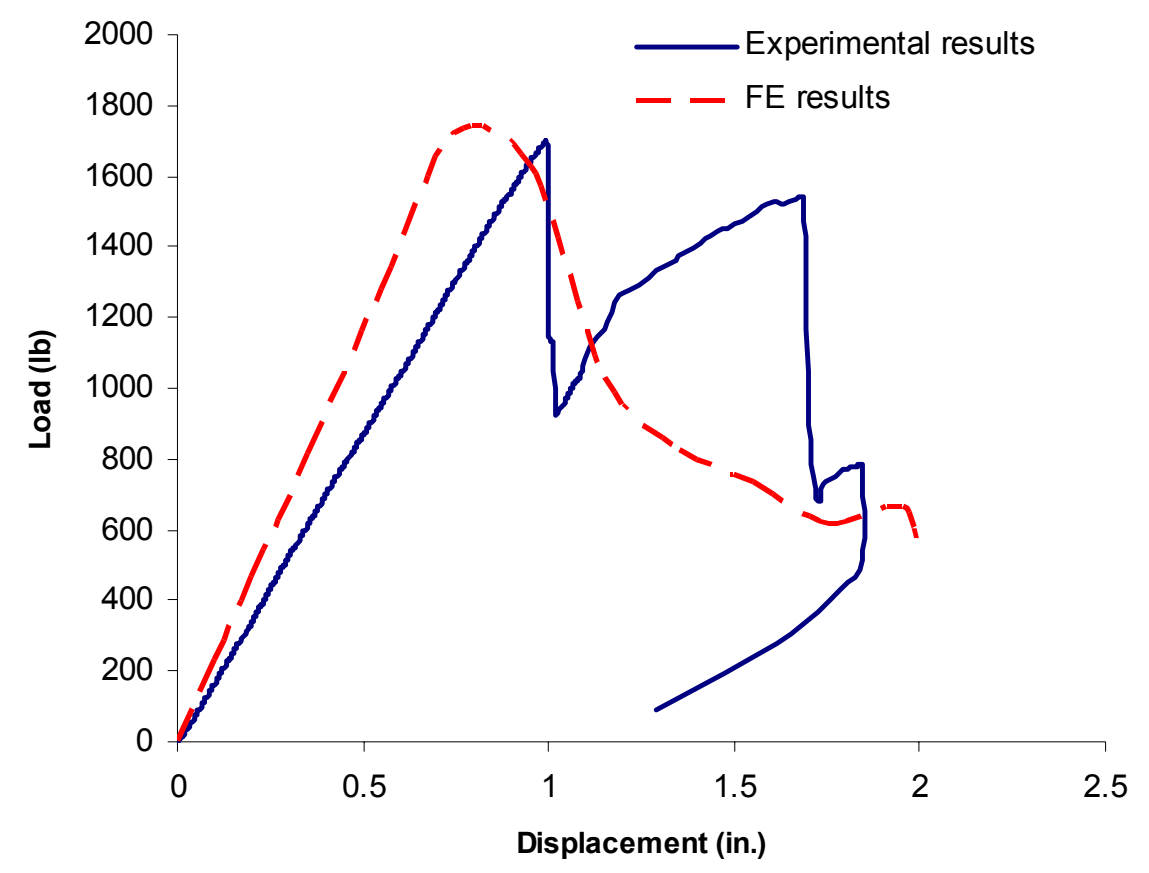

Figure 5.32 Load-displacement paths for Laminate 2 under bending

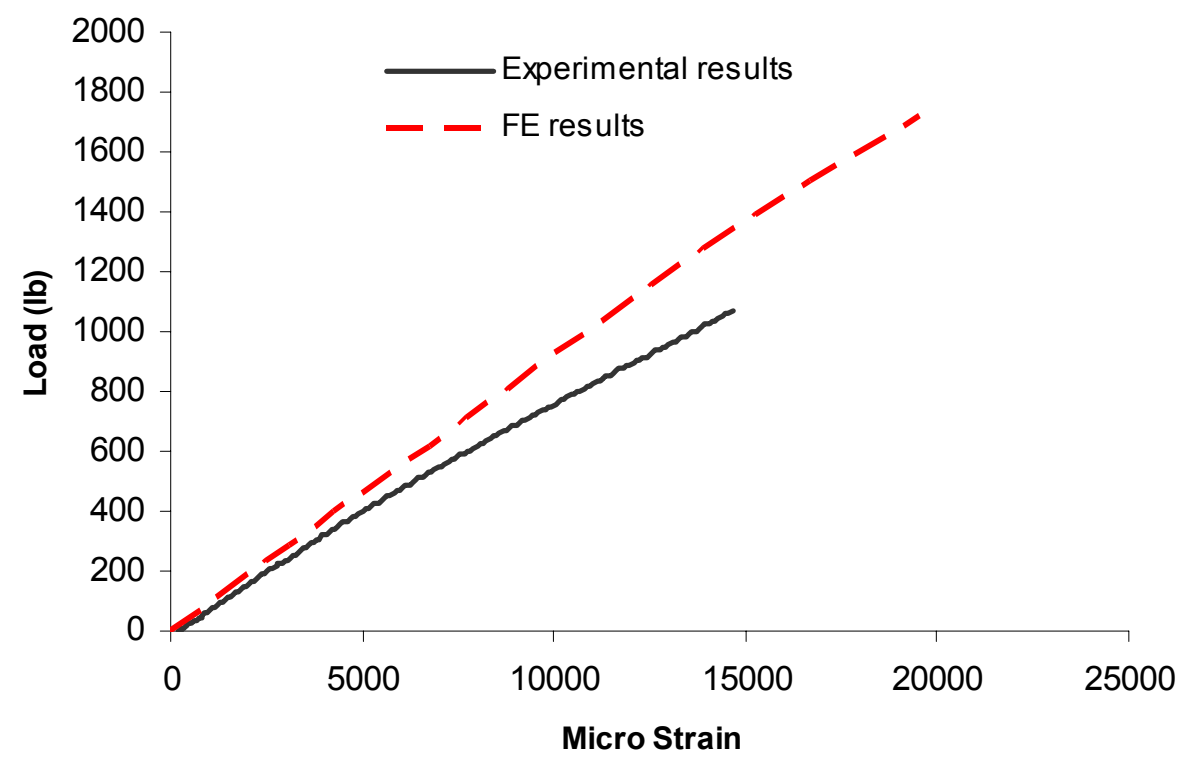

Figure 5.33 Load-strain paths for Laminate 2 under bending 


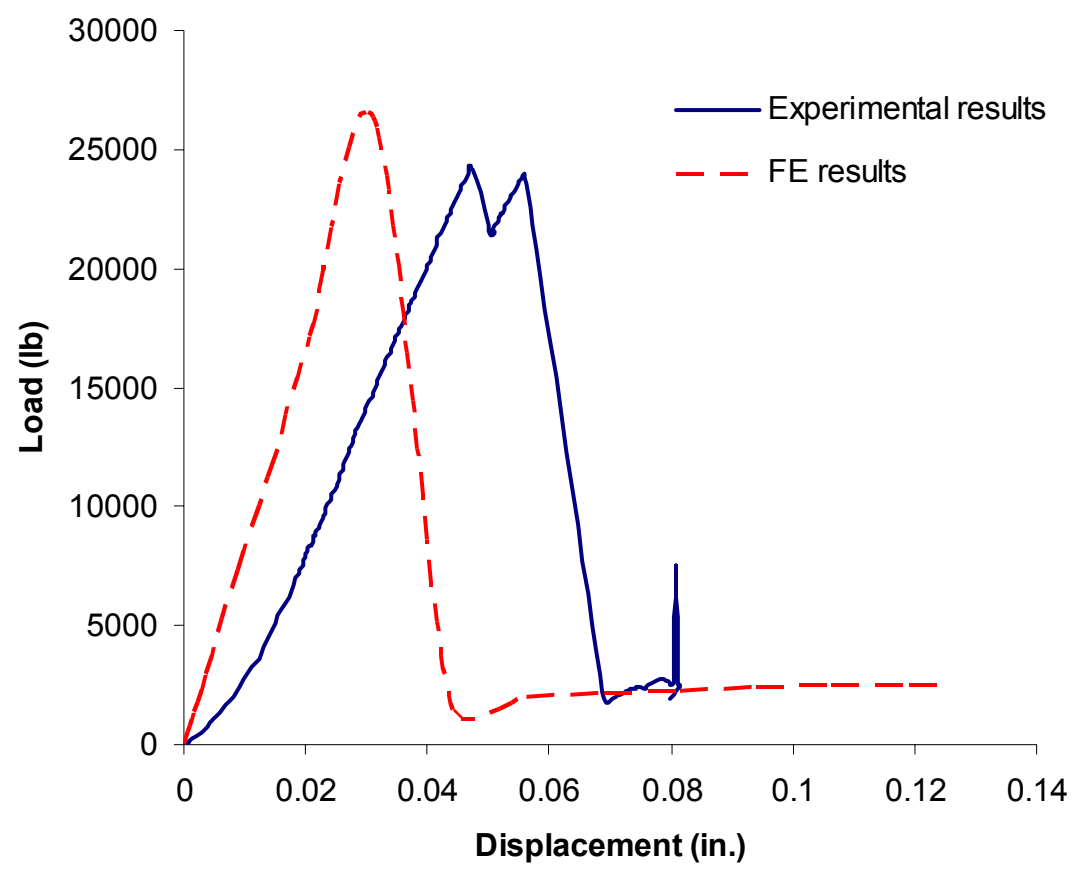

Figure 5.34 Load-displacement paths for Laminate 1L under compression

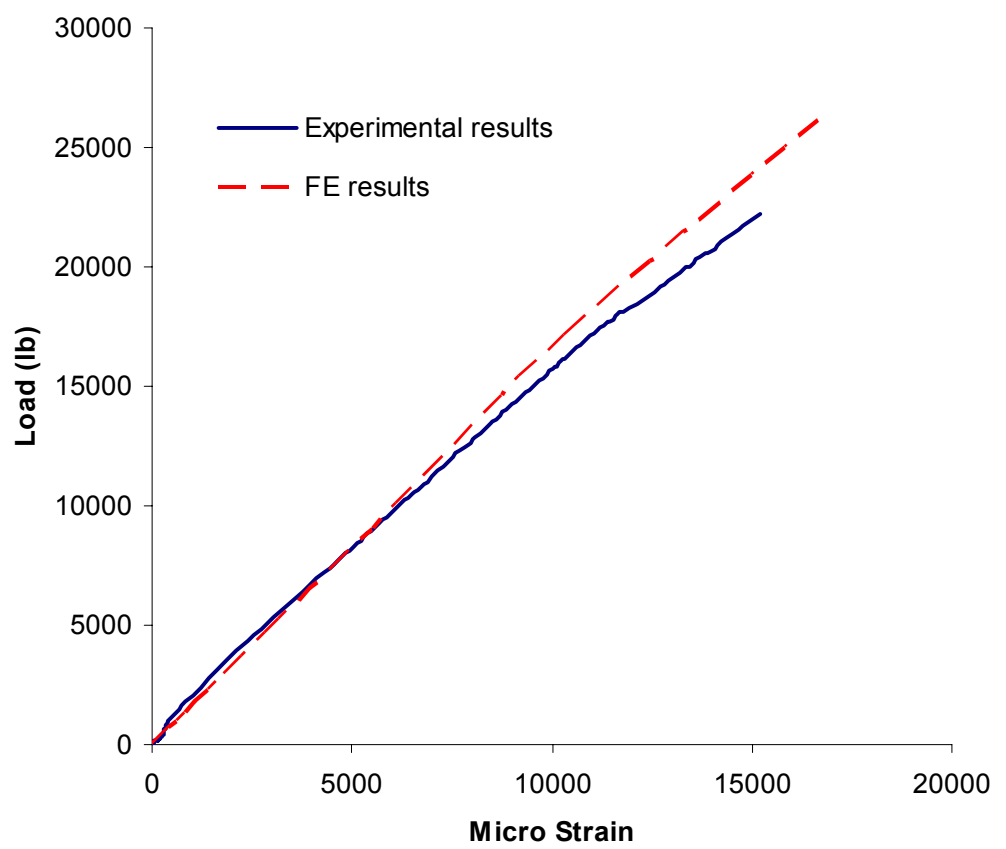

Figure 5.35 Load-strain paths for Laminate 1L under compression 


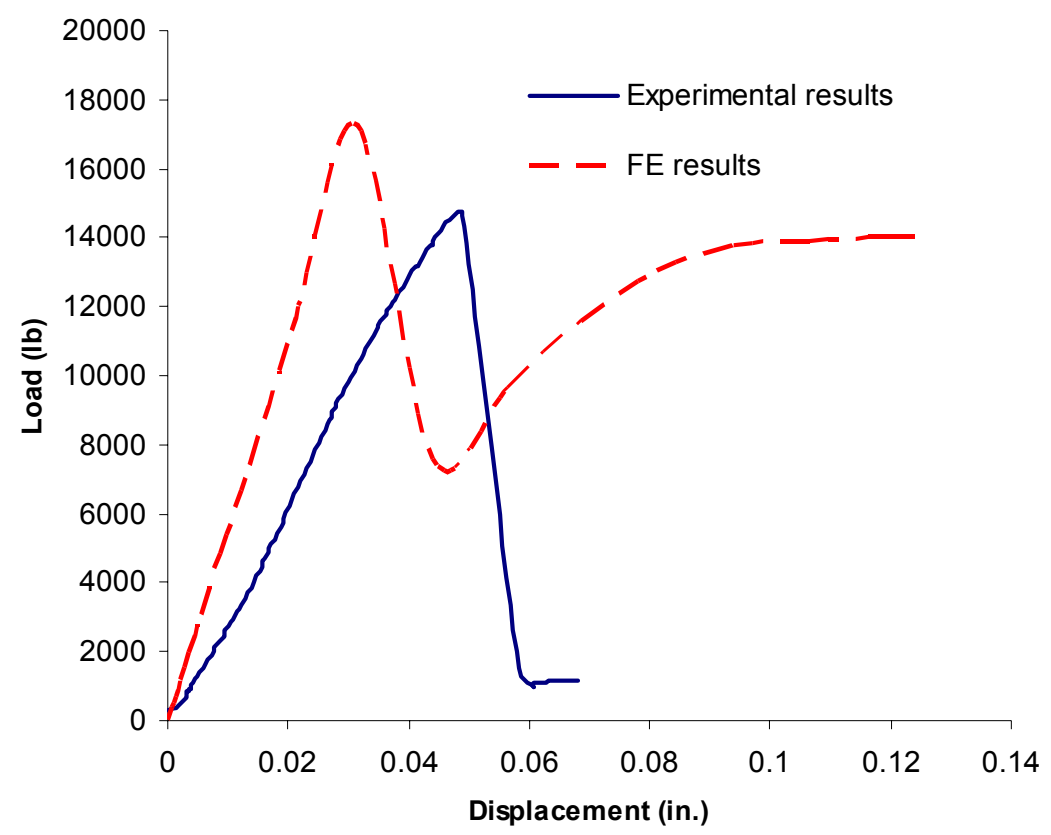

Figure 5.36 Load-displacement paths for Laminate 1T under compression

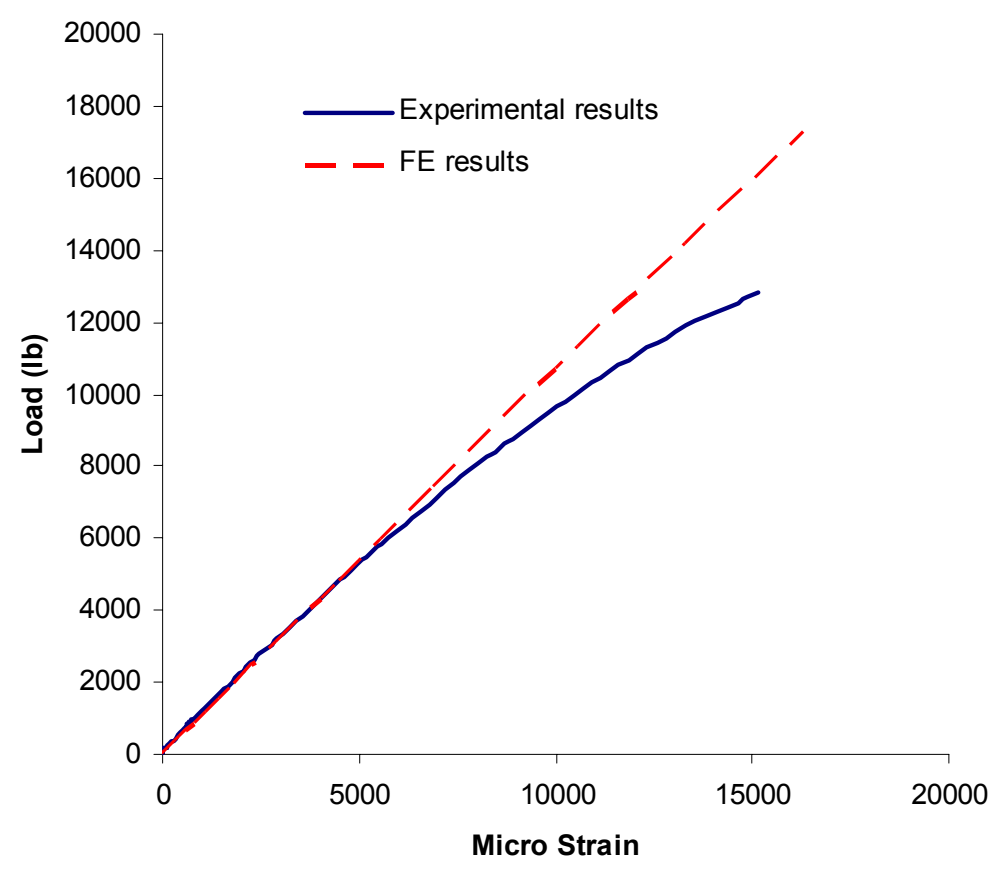

Figure 5.37 Load-strain paths for Laminate 1T under compression 


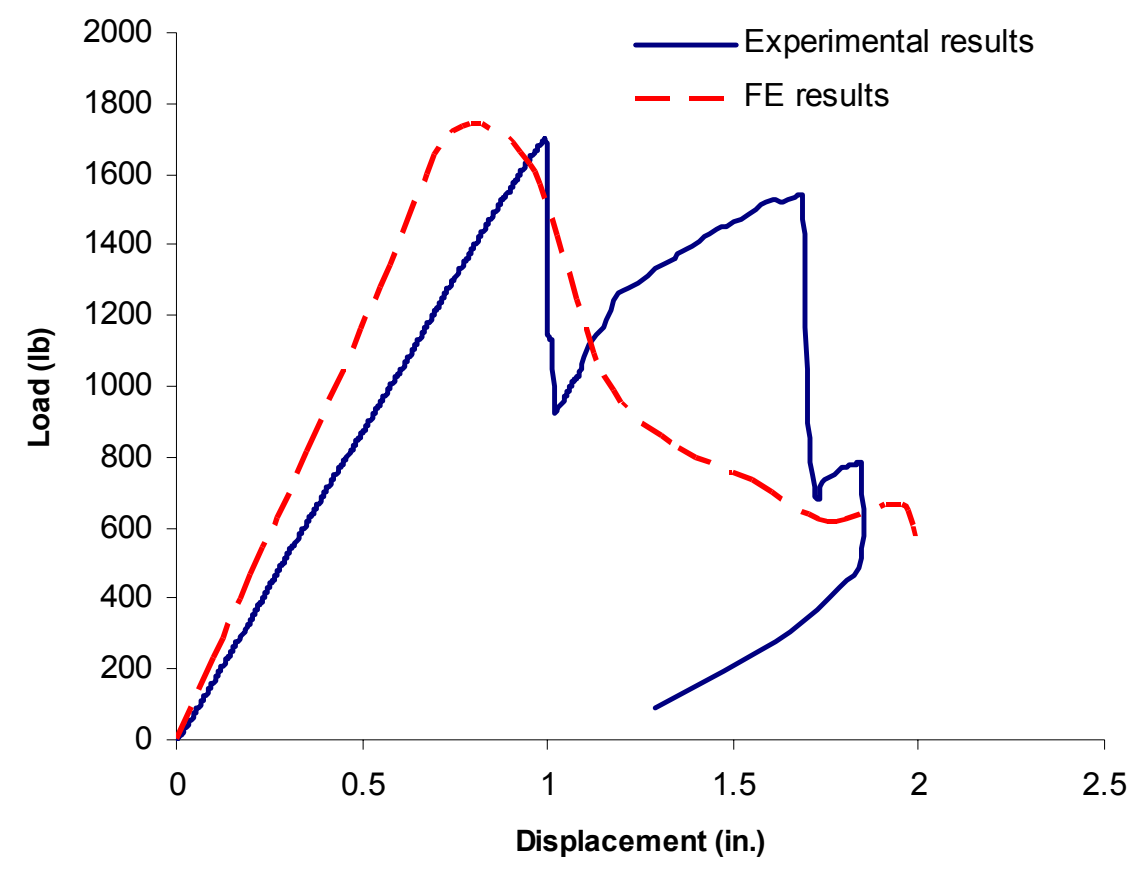

Figure 5.38 Load-displacement paths for Laminate 2 under compression

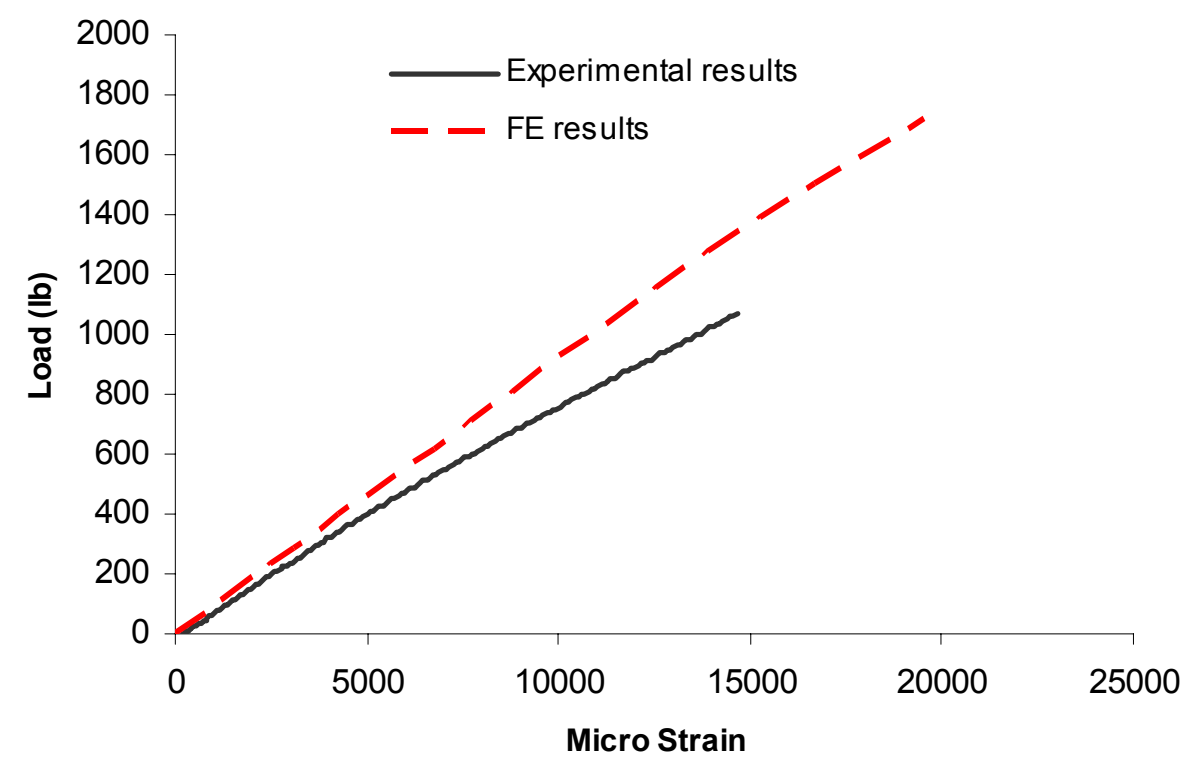

Figure 5.39 Load-strain paths for Laminate 2 under compression 


\section{CHAPTER 6}

\section{DESIGN GUIDELINES AND RECOMMENDED IMPROVEMENTS}

\subsection{Step-by-step Design Guidelines ${ }^{*}$}

\subsubsection{Out-of-plane compression}

Fig. 6.1 can be used to predict compression failure load. The following method is proposed:

1) Compare the height of the panel $h$ with transition height $h_{T}$, as shown in Table 6.1. If $h<h_{T}$, the failure mode is pure compression failure, and go to step 2 to calculate controlling strength; otherwise, buckling dominates the failure, and use strength provided in step 3;

2) The compressive strength corresponding to pure compression failure can be calculated using

$$
\sigma_{c}=\frac{F_{c}}{A}=\frac{f_{c} \times A_{c}}{A}
$$

\footnotetext{
* The units used in this chapter are $\mathrm{lb}$, inch, psi, and in-lb unless otherwise mentioned.
} 
where $f_{c}$ is the material compressive strength, $A_{c}$ is the total in-plane area of the core walls in a single cell, and $A$ is the in-plane area for a single cell. $F_{c}$ can also be determined from stabilized compression test, as demonstrated in this dissertation.

3) The buckling strength can be calculated using

$$
\sigma=\frac{A_{1} e^{\left(-h / t_{1}\right)}+A_{2} e^{\left(-h / t_{2}\right)}+F_{0}}{A}
$$

where $h$ is the height of the panel, and all the other parameters are listed in Table 6.2 .

4) Calculate the compressive stress based on the worst loading condition, and compare this stress with the compressive strength obtained from previous steps to find the safety factor.

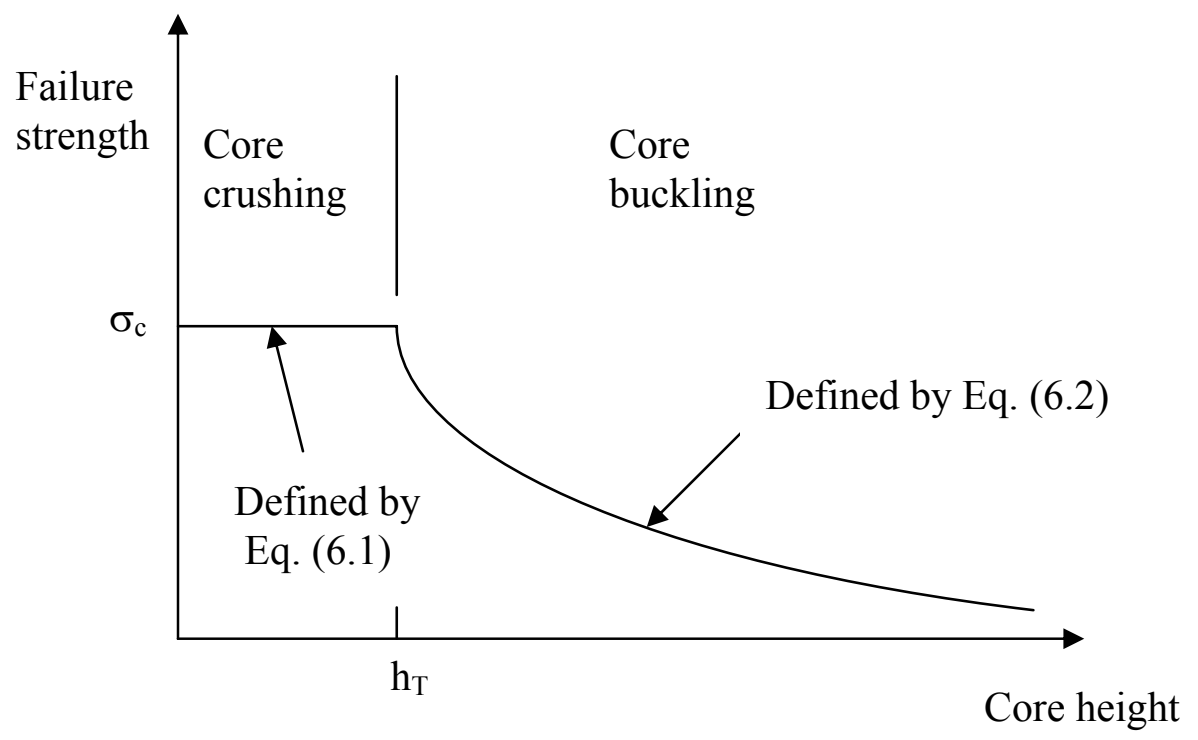

Figure 6.1 Design diagram 
Table 6.1 Transition height

\begin{tabular}{|c|c|c|c|}
\hline & One bonding layer & Two bonding layer & Three bonding layer \\
\hline $\mathrm{h}_{\mathrm{T}}$ & $1.25 \%$ & $1.42 ”$ & $1.5 \%$ \\
\hline
\end{tabular}

Table 6.2 Parameters for Eq. (6.2)

\begin{tabular}{|c|c|c|c|c|c|}
\hline & $\mathrm{A}_{1}$ & $\mathrm{t}_{1}$ & $\mathrm{~A}_{2}$ & $\mathrm{t}_{2}$ & $\mathrm{~F}_{0}$ \\
\hline One bonding layer & 957515 & 0.2363 & 124742 & 0.7464 & 8081 \\
\hline Two bonding layers & 87639 & 1.0105 & 954711 & 0.2917 & 8136 \\
\hline Three bonding layers & 1038189 & 0.2985 & 88384 & 1.0765 & 8152 \\
\hline
\end{tabular}




\subsubsection{Out-of-plane Shear}

Three distinct failure modes may occur for a panel under out-of-plane shear: shear crushing and shear buckling for the flat panel, and debonding for the curved panel, where the final failure load depends on the lowest value from these failure modes. Fig. 6.2 can be used to find failure strength for flat panel. As a result, the following method is proposed:

1) Compare the height of the panel $h$ with transition height $h_{T}$, as shown in Table 6.3. If $h<h_{T}$, the failure mode is pure shear failure, and use the material shear strength as controlling strength; otherwise, buckling dominates the failure, and use strength provided in step 2;

2) Shear buckling strength can be calculated using

$$
\tau=\frac{A_{1}}{t} e^{-\frac{R}{t_{1}}}+\frac{A_{2}}{t} e^{-\frac{R}{t_{2}}}+\frac{N_{0}}{t}
$$

where $R$ is the aspect ratio, $t$ is the thickness of the core wall, and all the other parameters are given in Table 6.4.

3) Use the following equation to calculate equivalent shear modulus

$$
G_{x z}=45524+758 e^{-\frac{R-0.1113}{0.7987}}+143 e^{-\frac{R-0.1113}{20}}
$$


4) Calculate shear strain based on the equivalent shear modulus obtained from step

3 ;

5) Based on the shear strain from step 4, find the shear stress for flat panel. Compare this stress with the shear strength obtained from previous steps to get the safety factor;

6) Based on the shear strain obtained from step 4, find the interfacial tensile stress for curved panel using

$$
S_{22}=\gamma\left(240150-240150 e^{-(2.3172 h)^{1.0221}}\right)
$$

where $\gamma$ is the shear strain and $h$ is the height of the panel. Compare the interfacial tensile stress $\mathrm{S}_{22}$ with nominal interfacial tensile strength in Table 6.5 to find the safety factor.

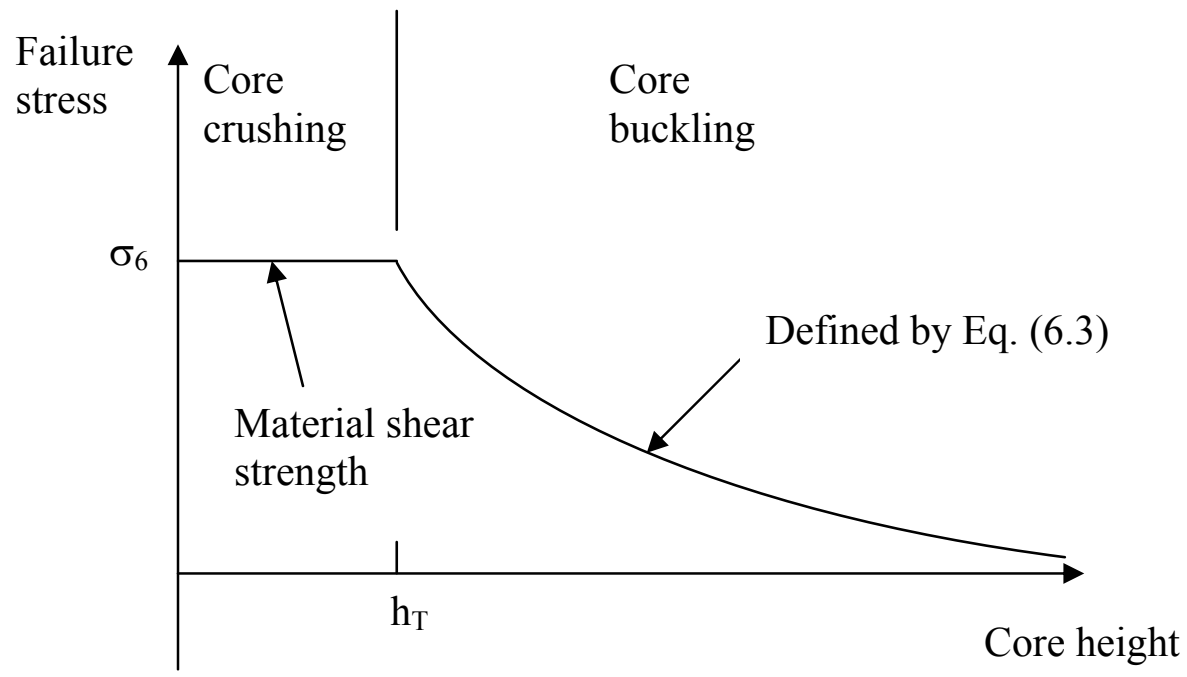

Figure 6.2 Design diagram 
Table 6.3 Transition height

\begin{tabular}{|c|c|c|c|}
\hline & One bonding layer & Two bonding layer & Three bonding layer \\
\hline $\mathrm{h}_{\mathrm{T}}$ & 3.48 ” & $3.72{ }^{\prime}$ & 3.84 \\
\hline
\end{tabular}

Table 6.4 Parameters for design equation

\begin{tabular}{|c|c|c|c|c|c|}
\hline & $\mathrm{A}_{1}$ & $\mathrm{t}_{1}$ & $\mathrm{~A}_{2}$ & $\mathrm{t}_{2}$ & $\mathrm{~N}_{0}$ \\
\hline One bonding layer & 2103 & 0.5326 & 34611 & 0.1388 & 448 \\
\hline Two bonding layers & 2661 & 0.5097 & 37093 & 0.1355 & 449 \\
\hline Three bonding layers & 3015 & 0.4970 & 38734 & 0.1339 & 450 \\
\hline
\end{tabular}

Table 6.5 Nominal interfacial tensile strength (psi)

\begin{tabular}{|c|c|c|c|c|c|c|c|}
\hline & B1C2 & B2C2 & B3C2 & B2C1 & B2C3 & B3C1 & B3C3 \\
\hline $\begin{array}{c}\text { Nominal interfacial } \\
\text { tensile strength }\end{array}$ & 1210 & 1754 & 2188 & 1279 & 1705 & 1713 & 3004 \\
\hline
\end{tabular}




\subsection{Example}

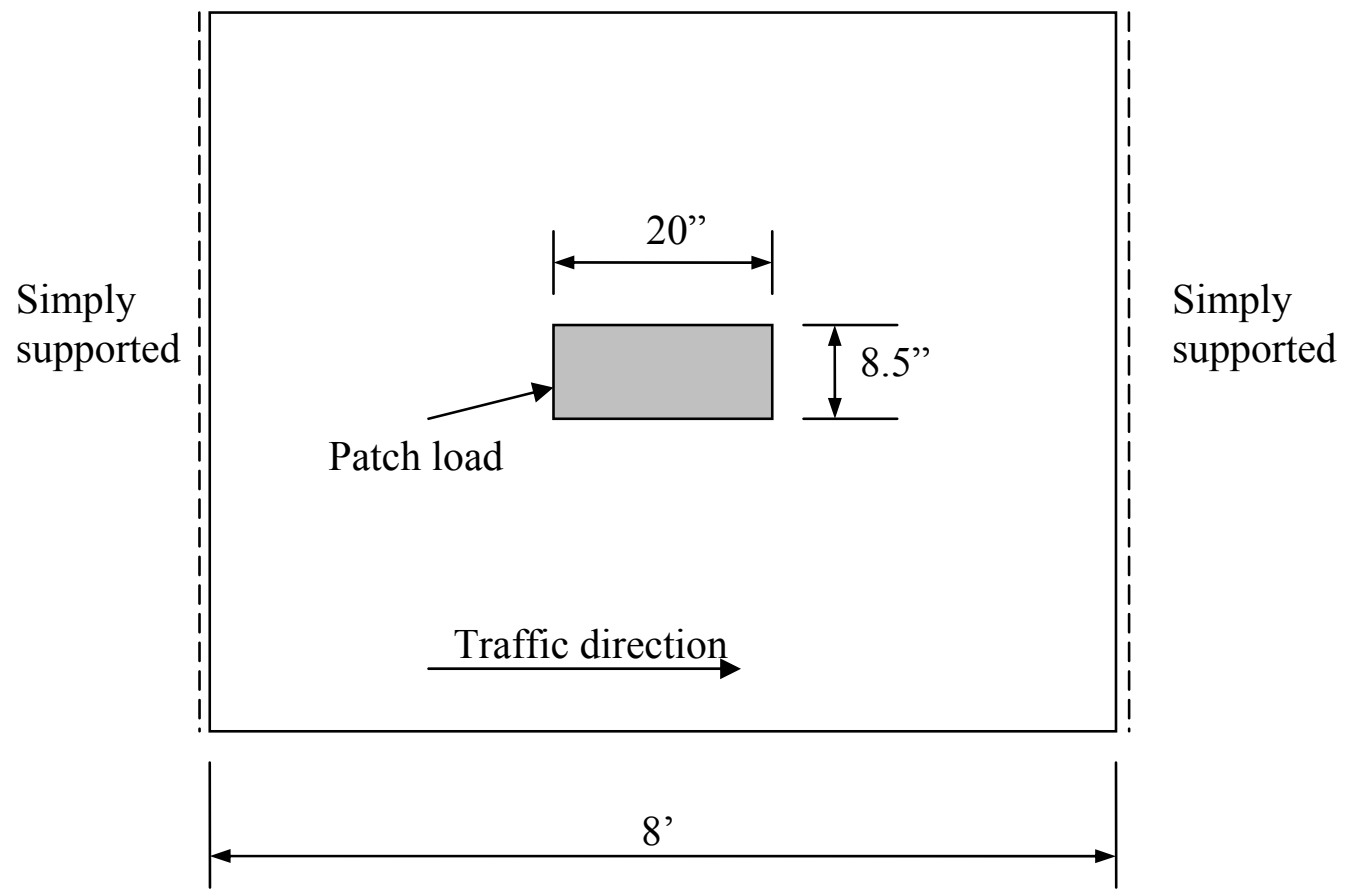

Figure 6.3 Panel layout

Using the design guidelines provided in Section 6.1, we can review the design of the HFRP sandwich panel for West Buckeye Bridge. The height of the panel $h=10$ ”, the core wall thickness $t=0.09$ " (two core thickness), the length for the flat panel $a=4$ ", and the aspect ratio $R=h / a=2.5$. One bonding layer is used at the interface between core and facesheet. The HFRP panel can be treated as one way slab, with two sides simplysupported and two sides free, as shown in Fig. 6.3. The maximum applied load, considering AASHTO HS20-44 (AASHTO, 1998) design truck wheel load with a dynamic load allowance of $33 \%$, is 


$$
P_{\max }=16,000 \times 1.33=21,280 \quad l b
$$

According to AASHTO LRFD Specifications (AASHTO, 1998), the length of the contact area between wheel and bridge deck is 20 ", and the width can be calculated as

$$
\ell=Y(1+I M / 100) P / 2.5=1.0(1+33 / 100) 16 / 2.5=8.512 \quad \text { in }
$$

(AASHTO Eq. 3.6.1.2.5-1) (6.7)

where $Y$, the load factor, is assumed to be 1.0 for safety reasons; $I M=$ dynamic allowance percent; and $P=16.0$ kips for the design truck.

\subsubsection{Compressive Strength}

Table 6.6 Compressive strength check

\begin{tabular}{|c|c|c|c|c|}
\hline $\begin{array}{c}\text { Core height } \\
\text { (in.) }\end{array}$ & $\begin{array}{c}\text { Pure compressive } \\
\text { strength (psi) }\end{array}$ & $\begin{array}{c}\text { Buckling } \\
\text { strength (psi) }\end{array}$ & $\begin{array}{c}\text { Controlling } \\
\text { strength (psi) }\end{array}$ & $\begin{array}{c}\text { Safety } \\
\text { factor }\end{array}$ \\
\hline 0.5 & 2313 & 11708 & 2313 & 18.5 \\
\hline 2 & 2313 & 1052 & 1052 & 8.4 \\
\hline 4 & 2313 & 542 & 542 & 4.3 \\
\hline 8 & 2313 & 505 & 505 & 4.0 \\
\hline 10 & 2313 & 505 & 505 & 4.0 \\
\hline 12 & 2313 & 505 & 505 & 4.0 \\
\hline 20 & 2313 & 505 & 505 & 4.0 \\
\hline 40 & 2313 & 505 & 505 & 4.0 \\
\hline
\end{tabular}


The compressive stress can be calculated as

$$
\sigma=\frac{P_{\max }}{8.512 \times 20}=125 \quad p s i
$$

The panel height for West Buckeye Bridge is 10". From Table 6.1, we find that the transition height is 1.25 " for one bonding layers, which is less than 10 ", and therefore, buckling controls the design. Substituting all the values into Eq. (6.2), the buckling strength is found to be $505 \mathrm{psi}$, which gives a safety factor of 505/125=4.0. The calculation can be repeated for other core heights, and the results are given in Table 6.6. 


\subsubsection{Shear Strength}
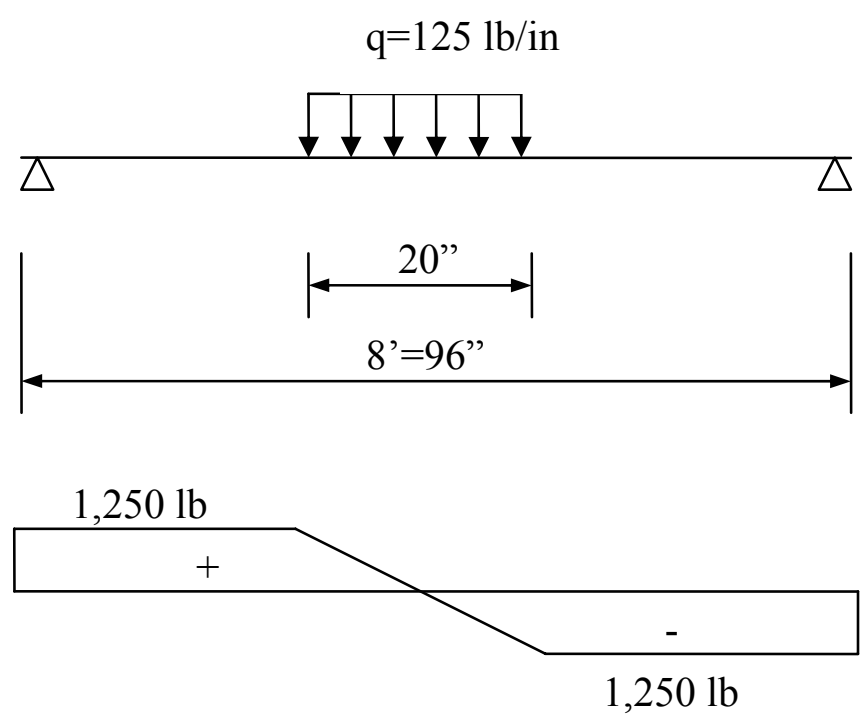

Shear diagram

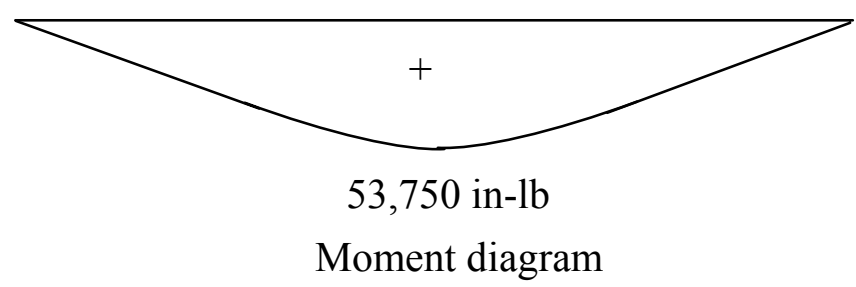

Figure 6.4 Unit width panel loading condition

To be conservative, we take out 1 " wide beam with a distributed load q acting at mid-span, as shown in Fig. 6.4. The distributed load q can be calculated as

$$
q=\frac{p_{\max }}{20 \times 8.512}=125 \quad \mathrm{lb} / \mathrm{in}
$$

The shear and moment diagrams are given in Fig. 6.4. 
The height of the panel is 10". Using Eq. (6.4), we can find the equivalent shear stiffness $G_{x z}=45,689 \mathrm{psi}$. Then the shear strain can be calculated as

$$
\gamma=\frac{V}{G_{x z} h}=\frac{1250}{45689 \times 10}=0.002736
$$

It is noted that the shear strain for the flat panel is the same as the global shear strain. Therefore, the shear stress in the flat panel is

$$
\tau_{1}=G_{12} \gamma=0.61 \times 10^{6} \times 0.002736=1669 \quad \text { psi }
$$

where $G_{12}$ is the material shear modulus from Table 3.1. From Table 6.3, we find $h_{T}=3.48$ ". Apparently $h>h_{T}$, and buckling controls the design. From Eq. (6.3), the shear buckling strength is found to be 5,074 psi, giving a safety factor of 3.0.

To find whether it delaminates, the shear strain is substituted into Eq. (6.5), and the interfacial shear stress is found to be 657 psi. Comparing with the nominal interfacial strength of 1,210 psi from Table 6.5, it gives a safety factor of 1.84 .

The procedures can be repeated for other core heights, as shown in Table 6.7. 
Table 6.7 Shear strength check

\begin{tabular}{|c|c|c|c|c|c|c|c|c|c|c|}
\hline $\begin{array}{c}\text { Height } \\
\text { (in.) }\end{array}$ & $\begin{array}{c}\text { Aspect } \\
\text { ratio R }\end{array}$ & $\begin{array}{c}\text { Shear } \\
\text { stiffness } \\
\text { (psi) }\end{array}$ & $\begin{array}{c}\text { Shear } \\
\text { strain }\end{array}$ & $\begin{array}{c}\text { Shear } \\
\text { stress in } \\
\text { flat panel } \\
\text { (psi) }\end{array}$ & $\begin{array}{c}\text { Buckling } \\
\text { strength } \\
\text { (psi) }\end{array}$ & $\begin{array}{c}\text { Material } \\
\text { strength } \\
\text { (psi) }\end{array}$ & $\begin{array}{c}\text { Controlling } \\
\text { strength } \\
\text { (psi) }\end{array}$ & $\begin{array}{c}\text { Safety } \\
\text { factor } \\
\text { for flat } \\
\text { panel }\end{array}$ & $\begin{array}{c}\text { Interfacial } \\
\text { normal } \\
\text { stress (psi) }\end{array}$ & $\begin{array}{c}\text { Safety } \\
\text { factor for } \\
\text { curved } \\
\text { panel }\end{array}$ \\
\hline 0.5 & 0.125 & 46412 & 0.026933 & 16429 & 179670 & 10239 & 10239 & 0.62 & 4445 & 0.27 \\
\hline 2 & 0.5 & 46130 & 0.013549 & 8265 & 24587 & 10239 & 10239 & 1.24 & 3227 & 0.37 \\
\hline 4 & 1 & 45910 & 0.006807 & 4152 & 8838 & 10239 & 8838 & 2.13 & 1635 & 0.74 \\
\hline 8 & 2 & 45725 & 0.003417 & 2084 & 5525 & 10239 & 5525 & 2.65 & 821 & 1.47 \\
\hline 10 & 2.5 & 45689 & 0.002736 & 1669 & 5192 & 10239 & 5192 & 3.11 & 657 & 1.84 \\
\hline 12 & 3 & 45668 & 0.002281 & 1391 & 5062 & 10239 & 5062 & 3.64 & 548 & 2.21 \\
\hline 20 & 5 & 45638 & 0.001369 & 835 & 4980 & 10239 & 4980 & 5.96 & 329 & 3.68 \\
\hline 40 & 10 & 45611 & 0.000685 & 418 & 4978 & 10239 & 4978 & 11.91 & 165 & 7.35 \\
\hline
\end{tabular}




\subsubsection{Facesheet check}

As composite action is not allowed for FRP bridge decks, the bending moment is carried through the membrane forces of the facesheet, as shown in Fig. 6.5. From Fig. 6.4, we have

$$
M=53,750 \text { in- } l b
$$

And the compressive and tensile force can be calculated as

$$
C=T=\frac{M}{h}=\frac{53750}{10}=5375 \quad l b
$$

From Chapter 5, the compressive strength for the facesheet with current configuration is $36,670 \mathrm{lb}$, giving a safety factor of 4.9. Safety factor for the other laminates are given in Table 6.8, from which we can see that, even when ChSM layer is introduced into the facesheet, compressive strength of facesheet is still not a concern for design.

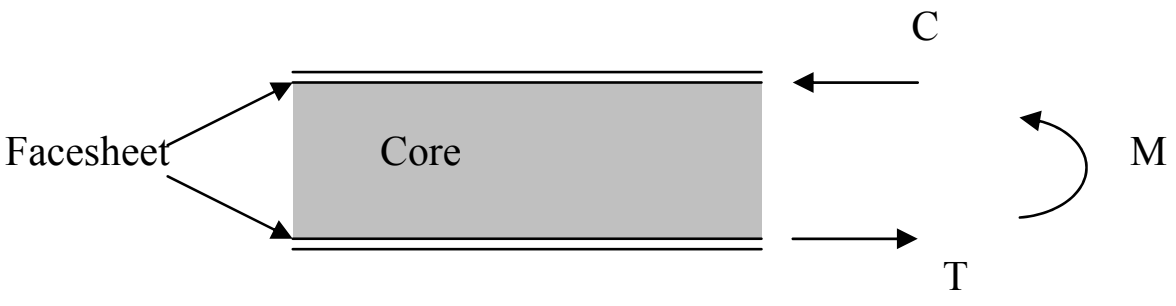

Figure 6.5 Forces acting on facesheet 
Table 6.8 Facesheet check

\begin{tabular}{|c|c|c|c|c|}
\hline & Laminate 1L & Laminate 1T & Laminate 2 & Laminate 3 \\
\hline Failure load (lb) & 26670 & 13931 & 23391 & 21903 \\
\hline Safety factor & 4.9 & 2.6 & 4.4 & 4.1 \\
\hline
\end{tabular}

In conclusion, the current panel configurations are sufficient for the design. It is also interesting to find that several panel heights, as shown in Table 6.7 in shaded area, will fail due to delamination.

\subsection{Recommended Improvements}

\subsubsection{Compression Behavior}

From the conclusions drawn above, it is found that the bonding effect is negligible for improving the buckling capacity of the panels, since the actual panel height is larger than 4" in most cases. Therefore, an effective way to improve performance is to increase the thickness of the flat panel. We recommend that the flat panel be composed of 6.0 $\mathrm{oz} / \mathrm{ft}^{2}$ mat while the curved panel be made of $4.5 \mathrm{oz} / \mathrm{ft}^{2}$ mat. The buckling stresses for current and recommended configurations are listed in Table 6.9.

Table 6.9 Comparison between current and recommended configuration

\begin{tabular}{|c|c|c|c|c|}
\hline Core height & $\begin{array}{c}\text { Buckling load } \\
\left(\text { lb/in }{ }^{2}\right) \\
(\text { Current })\end{array}$ & Safety factor & $\begin{array}{c}\text { Buckling load } \\
\left(\text { lb/in }{ }^{2}\right) \\
(\text { Recommended })\end{array}$ & Safety factor \\
\hline $2 "$ & 1,052 & 8.4 & 2,493 & 19.9 \\
\hline $4 "$ & 542 & 4.3 & 1,285 & 10.3 \\
\hline $8 "$ & 505 & 4.0 & 1,197 & 9.6 \\
\hline $20 "$ & 505 & 4.0 & 1,197 & 9.6 \\
\hline $40 "$ & 505 & 4.0 & 1,197 & 9.6 \\
\hline
\end{tabular}




\subsubsection{Shear Behavior}

Since all the specimens failed due to delamination and not material failure except for the specimens with excessive bonding layers, which is seldom adopted in practice; i.e., neither shear nor compression of the core was observed, the effective way to increase the bending strength is to improve the bonding effect, i.e., for example, by increasing the contact area between the core panel and bonding layer. Possible ways to manufacture the bonding joints are as shown in Fig. 6.6. Since no model is available to quantify the bonding strength, the flatwise tension test and interface shear test are recommended to evaluate the bonding effect.
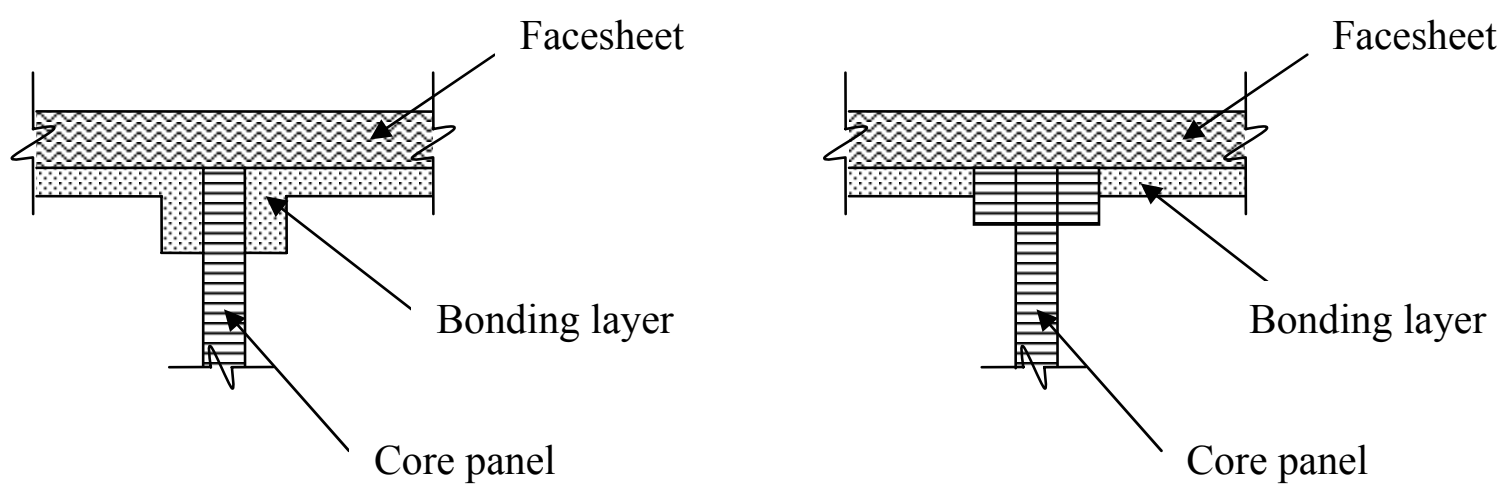

Figure 6.6 Recommended joints

\subsubsection{Facesheet Study}

From the results we obtained so far, the failure of facehsheet is not the dominant failure mode. However, if we introduce ChSM layer into the facesheet, it may reduce the stiffness, resulting in larger deflection. Then a problem may arise for the polymer 
concrete layer bonded to bridge deck, as the polymer concrete may crack and debond due to larger deflections. A further study needs to be conducted on this topic.

If the polymer concrete is not of concern, we can use the configuration Laminate 2, as described in Chapter 5. If the larger deflection is a problem, one way to offset the stiffness loss is to increase the thickness of the facesheet. Of course the total cost should be considered for this alternative, which can be evaluated through an optimization study. 


\section{CHAPTER 7}

\section{CONCLUSIONS AND RECOMMENDATIONS}

In this dissertation, a comprehensive study is carried out on strength evaluation of HFRP sandwich panels with sinusoidal core geometry. Specifically, the behavior of sandwich panels for out-of-plane compression, out-of-plane shear and facesheet are studied through a combination of analytical solution and experimental investigation. Major findings and conclusions are presented in this chapter, followed by suggestions for future work.

\subsection{Conclusions}

\subsubsection{Out-of-plane Compression}

Two analytical models, corresponding to pure compression and elastic buckling failure, respectively, are provided for panels subject to out-of-plane compression. The facesheet and core are attached by contact molding and are, therefore, not rigidly connected. Thus, the buckling problem can be described as the instability of an FRP core 
panel with two rotationally restrained loaded edges. By solving a transcendental equation, the critical compression buckling stresses are obtained, and a simplified expression to predict buckling strength is formulated in terms of the elastic restraint coefficient. Unlike existing solutions for eigenvalue analysis, where the number of half waves should be predefined when calculating the buckling load, this solution can give the minimum buckling load and the corresponding number of half waves. The accuracy is verified by FE and experimental results.

An elastic restraint coefficient is introduced to quantify the bonding layer effect between the facesheet and core, which plays an important role on the buckling behavior. However, rigid connection is commonly used in the analysis of sandwich structures, corresponding to $\zeta=0$ in this study. It is shown that a significant error may occur if the aspect ratio is within a certain limit. The coefficient of elastic restraint can be predicted using a simple and relatively accurate test method. Only two deflections are required to calculate this coefficient. Thus, the accuracy is increased. This method together with interface shear test and interface tension test can be act as the criteria to define the bonding quality of a given connection.

A parametric study is carried out by varying the core height. The result indicates that, if the core height is relatively low, there is a notable difference of the buckling stress for different number of bonding layers. The buckling stress decreases as the core height increases and reaches nearly a constant value once the core height reaches a certain limit. The buckling load is no longer sensitive to the bonding layer effect at this stage.

Compression tests were carried out to evaluate the effect of the bonding layer thickness and core thickness. Typically there are two failure modes for HFPR core under 
out-of-plane compression, buckling and pure compression failure, corresponding to bare and stabilized compression test, respectively. The buckling load is sensitive to the bonding layer effect. Specimens with three bonding layers fail at a higher load than those with one bonding layer. While for pure compression failure, the failure loads are not affected much by the number of bonding layers. The experimental results correlate closely with analytical and FE predictions.

Practical design equations are provided to calculate the compressive strength, which can be easily implemented. The method described can be further applied to other structures with elastic restraint at the loaded edges, like the web buckling in the steel girder.

\subsubsection{Out-of-plane Shear}

Analytical models, including delamination considering skin effect, shear crushing, and shear buckling are provided. The skin effect, composed of shear and bending warping, on the behavior of HFRP sandwich sinusoidal core panels, is for the first time investigated. Shear warping corresponds to cases with hinge connection between facesheet and core, and when both warping effects are considered, it corresponds to a rigid connection. Actual cases lie between these two conditions. A closed-form solution, based on proper description of displacement field at the interface, is derived considering shear warping, and FE analyses are carried out for both shear and bending warping cases. Accurate description of stiffness and stress distribution are obtained.

The skin effect is a localized phenomenon. The lower bound of the equivalent stiffness can thereby be adopted if the aspect ratio is high enough. However, it can 
significantly affect interfacial stress distribution, yielding a coupled stress state for the curved panel, where the normal stress may even be larger than the shear stress. This indicates, unlike the common belief that only shear stress occurs when the structure is under pure shear force, that tensile force at the interface arises, making it a potentially critical component. Therefore, special considerations are suggested for design purposes. The skin effects described herein only affect the stress distribution of the curved panel and has no effect on the flat panel. This effect on the stress distribution becomes less significant in the area away from the interface.

Rayleigh-Ritz method is employed to study the shear buckling of core panels with two sides elastically restrained. Based on analytical models, design formulas for delamination, shear buckling and shear crushing are proposed.

Four-point bending tests are carried out to study the HFPR panels under out-ofplane shear. Two cases of bending tests are carried out: longitudinal and transverse bending test. All specimens followed an approximate linear behavior prior to failure in bending. The failure load for the longitudinal specimens is much higher than that for the transverse specimens. For longitudinal samples, the excessive bonding layers specimens failed in shear, and the other specimen types failed by debonding. All of the transverse specimens failed by debonding. Transverse-type beams should be avoided when high shear stresses are expected. The failure load is sensitive to the bonding layer effect and core thickness effect. Specimens with more bonding layers and core thickness failed at a higher load than those with less bonding layer and core thickness. The failure load may vary for the same type of specimen due to the variability of bonding quality, which indicates the importance of quality control during manufacturing of the panels. 
The proposed design equations are used to predict the shear strength considering all failure modes. The predication agrees well with the test result, and can be used for design purposes.

\subsubsection{Facesheet Study}

A progressive failure model is developed using FE method to predict the behavior of laminated composite plates up to failure, where the accuracy is proved from the correlation between FE results and existing experimental data. It is much more efficient compared to 3-D model and offers great potential for a parametric study.

A parametric study is carried out on strength properties of the facesheet for a sandwich panel using this model on seven different configurations. Three variables are included: material properties, including bidirectional stitched fabrics, unidirectional layer of fiber roving and chopped strand mat; layer thickness; and layer sequences. The quality of each alternative is evaluated based on stiffness and strength performance. It is shown that, for a composite laminate, the compressive strength is lower than the tensile strength. If it is used for the facesheet of a sandwich bridge deck panel, as the top and bottom facesheet are in compression or tension with equal magnitude, the compressive strength of the facesheet is more critical and controls the design. If Chopped Strand Mat layer is introduced into the facesheet, the strength is not affected much, while the stiffness is reduced, resulting in a larger deflection under the same loading condition.

In order to further investigate the behavior of facesheet experimentally, coupon samples on selected configurations to evaluate compressive and bending strengths were tested in accordance with ASTM standards. The strength properties both in the 
longitudinal and transverse directions were evaluated. The dimensions of the coupon specimens vary for the different types of tests. The test results are also used to validate the progress failure model developed in this study and good correlation can be obtained. Through this combined test and analytical study, the strength properties of facesheet are obtained, which permit the optimization of facesheet design. It is found that the existing facesheet in industry is too conservative. Based on the results from this study, an optimized laminate is recommended for the future applications.

\subsubsection{Design Guidelines and Recommended Improvement}

Finally, step-by-step design guidelines are provided, where an example is given to illustrate its application. Recommendations to improve the strength of HFRP panels are presented. It is expected that this study will contribute to the design specifications and will accelerate the acceptance of this innovative light weight structure. All the methods presented in this dissertation can be extended to study other types of FRP sandwich structures.

\subsection{Suggestions for Future Work}

Although a comprehensive study on strength evaluation on HFRP sandwich panels is provided in this dissertation, further investigations are suggested in the following areas. 
Because FRP bridge decks are frequently used in structures subject to dynamic load, it is important that their response to fatigue be well known and understood. Up to now, there is no unified theory and all the studies are case specific. Therefore, it is recommended that fatigue test will be conducted on the specimens as described in this dissertation. The residual stiffness and strength can be used to evaluate the behavior, an S-N curve can be created based on the test data, and finally, a life prediction methodology can be proposed.

Skin effect can be decomposed into shear and bending warping effect, where an analytical solution is given for shear warping effect, and only FE results are provided for additional bending warping. Further study needs to be done to give a closed-form solution for bending warping, where out-of-plane deformation should be properly described.

All the design equations provided in this dissertation are verified using the coupon tests as described in this dissertation. Full-scale testing, although more costly, are suggested to continue experimental investigations. The results can be used to correlate with the existing design equations.

Only static loading is considered in this study, other effects, such as dynamic loading, thermal effects, and durability studies are also very important areas that need to be worked on. 


\section{PUBLICATIONS DURING THE STAGE OF PH.D. STUDY}

Chen, A. and Davalos, J.F. (2004). "A Solution including Skin Effect for Stiffness and Stress Field of Sandwich Honeycomb Core", submitted to International Journal of Solids and Structures, in review.

Davalos, J.F. and Chen, A. (2004). "Buckling Behavior of Honeycomb FRP Core with Partially Restrained Loaded Edges under Out-of-plane Compression", submitted to Journal of Composite Materials, in review.

Chen, A. and Davalos, J.F. (2004). "Development of Facesheet for Honeycomb FRP Sandwich Bridge Deck Panels", Proceedings of $4^{\text {th }}$ International Conference on Advanced Composite Materials in Bridges and Structures, ACMBS IV, Calgary, Canada, July 20-23, 2004.

Chen, A. and Davalos, J.F. (2004). "Behavior of Honeycomb FRP Sandwich Sinusoidal Core Panels with Skin Effect", Proceedings of $9^{\text {th }}$ ASCE Aerospace Division International Conference on Engineering, Construction and Operations in Challenging Environments, Houston, TX, March 7-10, 2004, pp. 625-632.

Chen, A. and Davalos, J.F. (2004). "Bonding and Aspect Ratio Effect on the Behavior of Honeycomb FRP Sandwich Cores", Proceedings of $8^{\text {th }}$ Pan American Congress of Applied Mechanics, PANCAM VIII, Havana, Cuba, January 5-9, 2004.

Chen, A. and Davalos, J.F. (2003). "Buckling of Honeycomb FRP Core with Partially Restrained Loaded Edges", Proceedings of Composites in Construction International Conference, Cosenza, Italia, September 16-19, 2003.

Chen, A. and Davalos, J.F. (2003). "Bending Strength of Honeycomb FRP Sandwich Beams with Sinusoidal Core Geometry", Proceedings of the Fourth CanadianInternational Composites Conference, CANCOM 2003, Ottawa, Canada, August 19-22, 2003.

Chen, A., Davalos, J.F., and Plunkett, J.D. (2002). "Compression Strength of Honeycomb FRP Core with Sinusoidal Geometry", Proceedings of the American Society for composites $17^{\text {th }}$ Technical Conference, West Lafayette, IN, October 21-23, 2002. 


\section{REFERENCES}

AASHTO (1998). Load Resistance and Factor Design, Bridge Design Specifications (2 ${ }^{\text {nd }}$ Edition), American Association of State Highway and Transportation Official, Washington, D.C.

ABAQUS User's Manual (version 6.3) (2002), HKS, Inc., RI, USA.

Adams, R.D., Maheri, M.R. (1993). "The dynamic shear properties of structural honeycomb materials", Composite Science and Technology, 47, pp. 15-23.

Allen, H.G. (1969). Analysis and Design of Structural Sandwich Panels. Pergamon Press, Oxford.

Allen, H.G. and Feng Zhengnong (1998). Mechanics of Sandwich Structures, Kluwer Academic Publisher, Netherlands, pp. 1-12.

ASTM Designation: C273-00, Standard Test Method for Shear Properties of Sandwich Core Materials (2000). ASTM, PA, USA.

ASTM Designation: C297-94, Standard Test Method for Flatwise Tensile Strength of Sandwich Constructions (2000). ASTM, PA, USA.

ASTM Designation: C365-00, Standard Test Method for Flatwise Compression Properties of Sandwich Cores (2000). ASTM, PA, USA.

ASTM Designation: C393-00, Standard Test Method for Flexure Properties of Sandwich Constructions (2000). ASTM, PA, USA.

ASTM Designation: D5379-98, Standard Test Method for Shear Properties of Composite Materials by the V-notched Beam Method (2000). ASTM, PA, USA.

ASTM Designation: D695-96, Standard Test Method for Compressive Properties of Rigid Plastics (2000). ASTM, PA, USA.

ASTM Designation: D790-99, Standard Test Method for Flexure Properties of Unreinforced and Reinforced Plastics and Electrical Insulating Materials (2000). ASTM, PA, USA.

Bakis, C.E., Bank, L.C., Brown, V.L., Cosenza, E, Davalos, J.F., Lesko, J.J., Machida, A., Rizkalla, S.H., and Triantafillou, T.C. (2002). "Fiber-reinforced Polymer Composites for Construction-State-of-Art Review", Journal of Composites for Construction, ASCE, 6(2), pp. 73-87. 
Barbero, E. J. (1999). Introduction to Composite Materials Design, Taylor \& Francis, Philadelphia, USA.

Barbero, E. J., Makkapati, S., and Tomblin, J. S. (1999). "Experimental Determination of Compressive Strength of Pultruded Structural Shapes", Composite Science and Technology, 59, pp. 2047-2054.

Barbero, E.J. and Raftoyiannis, I.G. (1993). "Local Buckling of FRP Beams and Columns", Journal of Material in Civil Engineering, ASCE, 5(3), pp.339-355.

Becker, W. (1998). "The in-plane stiffness of a honeycomb core including the thickness effect”, Arch. Appl. Mech., 68, pp. 334-341.

Bitzer, T.N. (1992). "Recent Honeycomb Core Developments", Proceedings of the Second International Conference on Sandwich Construction, Editors, D. Weissman-Berman and K-A. Olsson, EMAS Publications, United Kingdom, pp. 555-563.

Blackman, B.R.K., Hadavinia, H., Kinloch, A.J., and Williams, J.G. (2003). "The Use of a Cohesive Zone Model to Study the Fracture of Fiber Composites and Adhesively-bonded Joints", International Journal of Fracture Mechanics, 119, pp.25-46.

Bleich, F. (1952). Buckling strength of metal structures, McGraw-Hill, New York, USA.

Brown, Richard T. and Zureick, Abdul-Hamid (1998). "Truss Section Fiberglass Reinforced Composite Causeway Deck", Proceedings of $30^{\text {th }}$ International SAMPE Technical Conference, Oct. 20-24, pp. 303-315.

Burton, W. Scott and Noor, A.K. (1997). "Structual Analysis of the Adhesive Bond in a Honeycomb Core Sandwich Panel", Finite Elements in Analysis and Design, 26, pp. 213-227.

Caprino, G. and Langella, A. (2000). "Study of a Three-point Bending Specimen for Shear Characterisation of Sandwich Cores", Journal of Composite Materials, 34(9), pp. 791-814.

Chen, A. and Davalos, J.F. (2003). "Bending Strength of Honeycomb FRP Sandwich Beams with Sinusoidal Core Geometry", Proceedings of the fourth CanadianInternational Composites Conference, CANCOM 2003, Ottawa, Canada, August 19-22, 2003.

Chen, A. and Davalos, J.F. (2004). "A Solution including Skin Effect for Stiffness and Stress Field of Sandwich Honeycomb Core", submitted to International Journal of Solids and Structures, in review. 
Christensen, R.M. (1991). Mechanics of Composite Materials, Malabar: Krieger.

Cui, W.C., Wisnom, M.R., and Jones, M. (1992). "Failure Mechanism in Three and Four Point Short Beam Bending Tests of Unidirectional Glass/Epoxy", Journal of Strain Analysis, 27(4), pp. 235-243.

Cui, Weicheng and Wisnom, M.R. (1992). "A Combined Stress-based and Fracturemechanics-based Model for Predicting Delamiantion in Composites", Composites, 24(6), pp. 467-474.

Cvitkovich, M.K and Jackson, W.D. (1998). "Compression Failure Mechanisms in Composite Sandwich Structures", American Helicopter Society $54^{\text {th }}$ annual forum, Washington D.C., May 20-22 1998.

Daniel, M.E., Abot, L.J. (2000). "Fabrication, Testing and Analysis of Composite Sandwich Beams", Composite Science and Technology, 60, pp. 2455-2463.

Davalos, J.F., Qiao, P., Xu, X.F., Robinson, J., and Barth, K.E (2001). "Modeling and Characterization of Fiber-reinforced Plastic Honeycomb Sandwich Panels for Highway Bridge Applications", Composite Structures, 52, pp 441-452.

DeTeresa, S.J., Freeman, D.C., Groves, S.E., and Sanchez, R.E. (1999). "Failure under multiaxial stresses of component materials for fiber composite sandwich construction", Proceedings of Twelfth International Conferences on Composite Materials (ICCM-12), July 5-9, 1999, Paris, France, pp. 198

Echaabi, Jamal, Trochu, Francois, Gauvin, Raymond (1996). "Review of Failure Criteria of Fibrous Composite Materials”, Polymer Composites, 17(6), pp. 786-798.

Elawadly, Khalil M. (2003). "On the Interlaminar Shear Stress Response for EGlass/Epoxy Composite”, Journal of Composite Materials, 37(23), pp. 21492158.

El-Sayed, S. and Sridharan, S. (2002). "Cohesive Layer Model for Predicting Delamination Growth and Crack Kinking in Sandwich Structures", International Journal of Fracture Mechanics, 117, pp. 63-84.

Fairbairn, W. (1849). An Account of the Construction of the Britannia and Conway Tubular Bridges, John Weale, London

FEMAP User's Manual (version 8.1) (2001). Enterprise Software Products, Inc., PA, USA.

Fortes, M.A., Ashby, M.F. (1999). "The Effect of Non-uniformity on the In-plane Modulus of Honeycombs", Acta Mater., 47(12), pp. 3469-3473. 
GangaRao, Hota V.S., Thippeswamy, Hemanth, K., Shekar, Vimala, and Craigo, Carl (1999). "Development of Glass Fiber Reinforced Polymer Composite Bridge Decks", SAMPE Journal, 35(4), pp. 12-24.

Gibson, J.L. \& Ashby, M.F. (1988). Cellular Solids, Structure and Properties, Pergamon Press, Oxford.

Grediac, M. (1993). "A Finite Element Study of the Transverse Shear in Honeycomb Cores”, Int. J. Solids Structures, 30(13), pp. 1777-1788.

Greif, R. and Chapon, E. (1993). "Investigation of Successive Failure Modes in Graphite/Epoxy Laminated Composite Beams", Journal of Reinforced Plastics and Composites, 12, pp. 602-621.

Hann, H.T. (1975). "On Approximation for Strength of Random Fiber Composites", Journal of Composite Materials, 9, pp. 316-326.

Halpin, J.C. and Kardos, J.L. (1978). "Strength of Discontinuous Reinforced Composites: I. Fiber Reinforced Composites", Polymer Engineering and Science, 18(6), pp. 496-504

Hashin, Z. (1980). "Failure Criteria for Unidiretional Fiber Composites", Journal of Complied Mechanics, 47, pp. 329-334.

Hohe, J. and Becker, W. (2001). "A Refined Analysis of the Effective Elasticity Tensors for General Cellular Sandwich Cores", Int. J. Solids Structures, 38, pp. 36893717.

Hwang, W.C. and Sun C.T. (1989). "Failure Analysis of Laminated Composites by Using Iterative Three-dimensional Finite Element Method", Computers and Structures, 33(1), pp. 41-47.

Kelsey, S., Gellatly, R.A., Clark, B.W. (1958). "The Shear Modulus of Foil Honeycomb Cores", Aircraft Eng., 30, pp. 294-302.

Kim, R. and Crasto, Y. (1992). "A longitudinal compression test for composites using a sandwich specimen”, Journal of Composite Materials, 26(13), pp. 1915-1929.

Kim, Youngchan (1995). A Layer-wise Theory for Linear and Failure Analysis of Laminated Composite Beams, Ph.D. Dissertation, Department of Civil and Environmental Engineering, West Virginia University, Morgantown, WV, USA

Kim, Youngchan, Davalos, J.F., and Barbero, E.J. (1996). "Progressive Failure Analysis of Laminated Composite Beams", Journal of Composite Materials, 30(5), pp. 536-560. 
Kollar, L.P. (2002). "Buckling of Unidirectional Loaded Composite Plates with One Free and One Rotationally Restrained Unloaded Edge", Journal of Structural Engineering, 128(9), pp. 1202-1211.

Kroll, Lothar and Hufenbach, Werner (1997). "Physically Based Failure Criterion for Dimensioning of Thick-walled Laminates", Applied Composite Materials, 4(5), pp. 321-332.

Kumar, Prakash, Chandrashekhara, K., and Nanni, Antonio (2003). "Testing and Evaluation of Components for a Composite Bridge Deck", Journal of Reinforced Plastics and Composites, 22(5), pp. 441-461.

Lee, H.S., Lee, J.R., and Kim, Y.K. (2002). "Mechanical Behavior and Failure Process During Compressive and Shear Deformation of Honeycomb Composite at Elevated Temperatures", Journal of Materials Science, 379(6), pp. 1265-1272.

Lee, James D. (1982). "Three Dimensional Finite Element Analysis of Damage Accumulation in Composite Laminate", Computers and Structures, 15(3), 1982, pp. 335-350.

Lingaiah, K. and Suryanarayana, B.G. (1991). "Strength and Stiffness of Sandwich Beams in Bending”, Experimental Mechanics, 3, pp. 1-7.

Lopez-Anido, R., Davalos, J.F., and Barbero, E.J. (1995). "Experimental Evaluation of Stiffness of Laminated Composite Beam Elements under Flexure", Journal of Reinforced Plastics and Composites, 14, pp. 349-361.

Luciano, R. and Barbero, E.J. (1994). "Formulas for the Stiffness of Composites with Periodic Microstructure", Int. J. Solids Struct., 31(21), pp. 2933-2944.

Makkapati, Satheesh (1994). Compressive Strength of Pultruded Structural Shapes, Master Thesis, Department of Mechanical and Aerospace Engineering, West Virginia University, Morgantown, WV, USA.

Masters, I.G., Evans, K.E. (1996). "Models for the Elastic Deformation of Honeycombs", Composite Structures, 35, pp. 403-422.

MDA (2003). http://www.mdacomsoites.org/psbridge_vehicular_print.html

Mouritz, A.P., Thomson, R.S. (1999). "Compression, Flexure and Shear Properties of a Sandwich Composite Containing Defects", Composite Structures, 44: pp. 263278.

Niu, Kangmin and Talreja, Ramesh (1998). "Modeling of Wrinkling in Sandwich Panels under Compression”, Journal of Engineering Mechanics, 125(8), pp. 875-883. 
Noor, A.K., Burton, W.S., and Bert, C.W. (1996). “Computational Models for Sandwich Panels and Shells", Appl. Mech. Rev., ASME, 49(3), pp. 155-199.

Ochoa, O.O. and Engblom, J.J. (1987). "Analysis of Progressive Failure in Composites", Composites Science and Technology, 28, pp. 87-102.

Papadopoulos, Lampros and Kassapoglou,Christos (2004). "Shear Buckling of Rectangular Composite Plates with Two Concentric Layups", Journal of Reinforced Plastics and Composites, 23 (1), pp. 5-16.

Penzien, J. and Didriksson, T. (1964). "Effective Shear Modulus of Honeycomb Cellular Structure", AIAA Journal, 2(3), pp. 531-535.

Plunkett, J.D. (1997). Fiber-reinforcement Polymer Honeycomb Short Span Bridge for Rapid Installation, IDEA Project Report.

Qiao, P. and Zou, G. (2003). "Local Buckling of Composite Fiber-reinforced Plastic Wide-flange Sections", Journal of Structural Engineering, 129(1), pp. 125-129.

Qiao, P., Davalos, J.F., and Brown, B. (2000). “A Systematic Approach for Analysis and Design of Single-span FRP Deck/Stringer Bridges”, Composites, Part B, 31(6-7), pp. 593-610.

Qiao, P., Davalos, J.F., and Wang, J. (2001). "Local Buckling of Composite FRP Shapes by Discrete Plate Analysis", Journal of Structural Engineering, 127(3), pp. 245255.

Reddy, J.N. (1999). Theory and analysis of elastic plates, Taylor \& Francis, Philadelphia, USA.

Reddy, Y.S. and Reddy, J.N. (1993). "Three Dimensional Finite Element Progressive Analysis of Composite Laminates Under Axial Extension", Journal of Composites Technology \& Research, JCTRER, 15(2), pp 73-87.

Shi, G. and Tong, P. (1995). Equivalent transverse shear stiffness of honeycomb cores, Int. J. Solids Structures, 32(10), pp. 1383-1393.

Spottswood, S.M. and Palazotto, A.N. (2001). "Progressive Failure Analysis of a Composite Shell”, Composite Structures, 53(1), pp 117-131.

Steenkamer, D.A. and Sullivan, J.L. (1999). "The Performance of Calcium Carbonate Filled, Random Fiber Composites”, Polymer Composites, 20(3), pp. 392-405.

Tan, S.C. (1991). "A Progressive Failure Model for Composite Laminates Containing Openings", Journal of Composite Materials, 25, pp. 556-577. 
Tan, S.C. and Perez, J. (1993). "Progressive Failure of Laminated Composite with a Hole under Compressive Loading”, Journal of Reinforced Plastics and Composites, 12, pp. 1043-1057.

Theotokoglou, E.E. (1996). "Analytical Determination of the Ultimate strength of Sandwich Beams", Applied Composite Materials, 3, pp. 345-353.

Thomson, R.S., Shah, Khan Z., and Mouritz, A.P. (1989). "Shear Properties of a Sandwich Composite Containing Defects", Composite Structures, 11, pp. 101120.

Timoshenko, S.P., and Gere, J.M. (1961). Theory of Elastic Stability, McGraw-Hill Book Company, Inc., NY, USA.

Tolson, S. and Zabaras, N. (1991). "Finite Element Analysis of Progressive Failure in Laminated Composite Plates", Computers and Structures, 38(3), pp. 361-376.

Tomblin, John S. (1994). Compressive Strength Models for Pultruded Glass Fiber Reinforced Composites, Ph.D. Dissertation, Department of Mechanical and Aerospace Engineering, West Virginia University, Morgantown, WV, USA.

Triantofillou, T.C. and Gibson, L.J. (1989). "Debonding in Foam-core Sandwich Panels", Mat. and Struct., 22., pp. 64-69.

Tsau, Li-Ren and Plunkett, Robert (1993). "Finite Element Analysis of Progressive Failure for Laminated FRP Plates with Inplane Loading", Engineering Fracture Mechanics, 45(4), pp. 529-546.

Ungsuwarungsri, T. and Knauss, W.K. (1987). "The role of Damage-soften Material Behavior in the Fracture of Composites and Adhesives", International Journal of Fracture Mechanics, 35, pp.221-241.

Vinson, J.R. (1999). The Behavior of Sandwich Structures of Isotropic and Composite Material, Technomic Publishing Company, Inc., PA, USA.

Vinson, J.R. and Sierakowski, R.L. (1987). The Behavior of Structures Composed of Composite Materials, Martinus Nijhoff Publishers, Dordrecht.

Waas, Anthony M. and Schultheisz, Carl R. (1996). "Compressive Failure of Composites, Part II: Experimental Studies”, Prog. Aerospace Sci., 32, pp. 43-78.

Wang, Weiqiao (2004). Cohesive Zone Model for Facesheet-Core Interface Delamination in Honeycomb FRP Sandwich Panels, Ph.D. Dissertation, Department of Civil and Environmental Engineering, West Virginia University, Morgantown, WV, USA. 
Warren, W.E., Kraynik, A.M. (1987). "Foam mechanics: the Linear Elastic Response of Two-dimensional Spatially Periodic Cellular Materials", Mech. Mater, 6, pp. 2737.

Xu, X.F. and Qiao, P. (2002). "Homogenized Elastic Properties of Honeycomb Sandwich with Skin Effect”, Int. J. Solids Structures, 39, pp. 2153-2188.

Xu, X.F., Qiao, Pizhong, and Davalos, Julio F. (2001). "Transverse Shear Stiffness of Composite Honeycomb Core with General Configuration", Journal of Engineering Mechanics, 127(11), pp. 1144-1151.

Zenkert, D. (1991). "Strength of Sandwich Beams with Interface Debondings", Composite Structures, 17, pp. 331-350.

Zenkert, D. (1995). An Introduction to Sandwich Construction, Chamelon Press, London.

Zhang, J. and Ashby, M.F. (1992). "Out-of-plane Properties of Honeycombs", International Journal of Mechanical Sciences, 34(6), pp. 475-489. 


\section{APPENDIX A \\ PROGRAM TO CALCULATE THE CRITICAL BUCKLING STRESS OF FRP PANELS WITH PARTIALLY RESTRAINED LOADED EDGES}

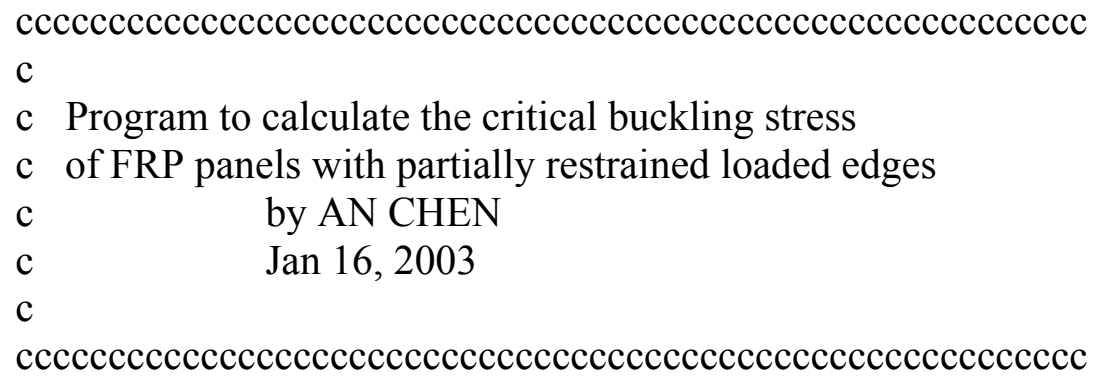

c Parametric study for gama with a fixed keshi

DATA $(\operatorname{gamad}(\mathrm{I}), \mathrm{I}=1,20) / 0.1,0.2,0.3,0.4,0.5,0.6,0.7,0.8$, $\$ 0.9,1 ., 2 ., 3 ., 4 ., 5 ., 6 ., 7 ., 8 ., 9 ., 10 .,-1 /$

c Parametric study for keshi with a fixed gama DATA (keshid(I), I=1,33) /0.0, 0.00001,0.01,0.02, 0.04, 0.06,0.08, $\$ 0.1,0.15,0.2,0.25,0.3,0.35,0.4,0.45$, 


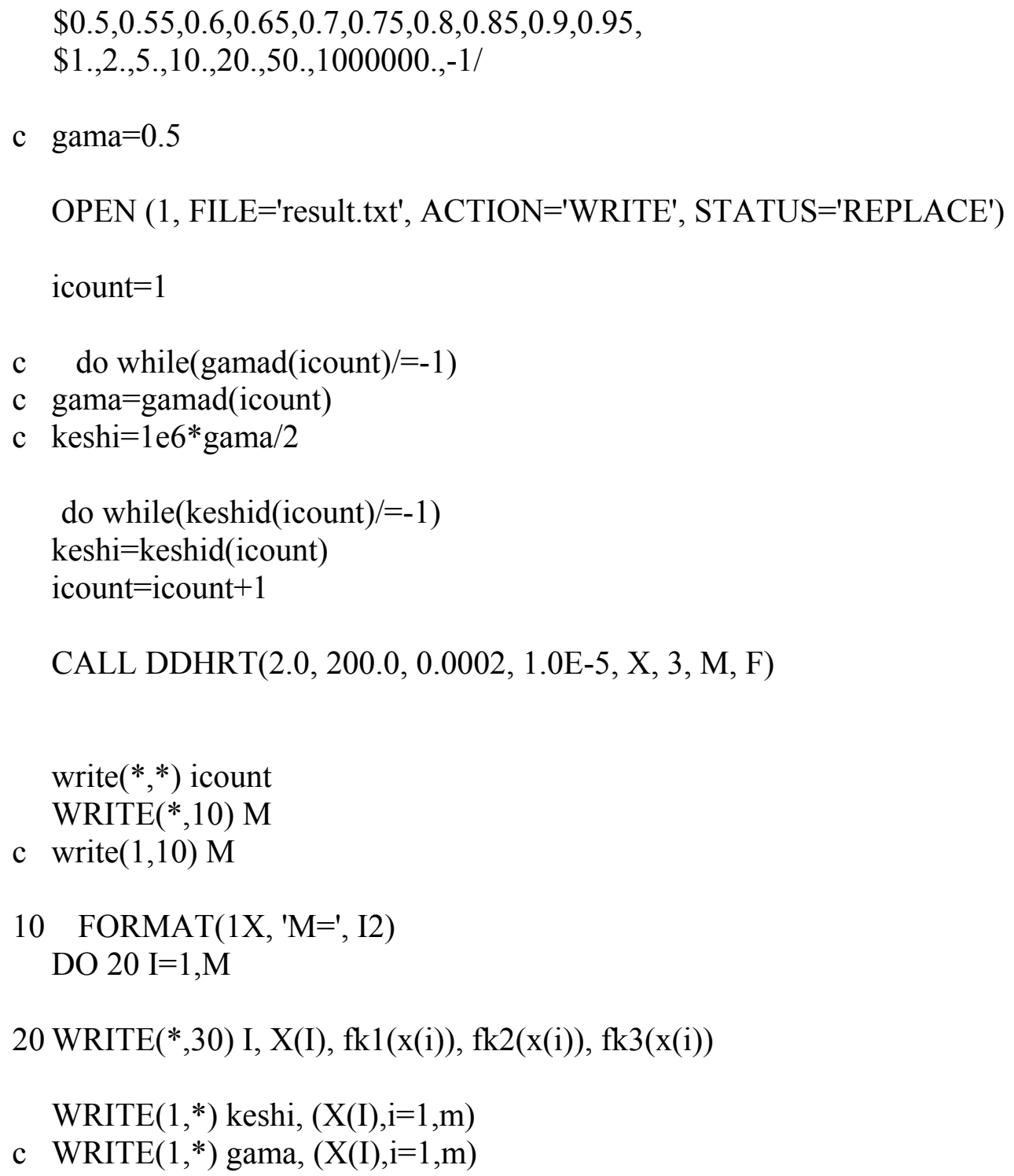

c See note for the defintion of functions

FUNCTION FK3(X)

$\mathrm{FK} 3=\mathrm{SQRT}((1-\mathrm{X}) * * 2-1)$

RETURN

END

FUNCTION FK1(X)

$\mathrm{FK} 1=\mathrm{SQRT}(-1+\mathrm{X}+\mathrm{FK} 3(\mathrm{X}))$ 


\author{
RETURN \\ END \\ FUNCTION FK2(X) \\ $\mathrm{FK} 2=\mathrm{SQRT}(-1+\mathrm{X}-\mathrm{FK} 3(\mathrm{X}))$ \\ RETURN \\ END
}

FUNCTION F(X)

real keshi

common /gama/ gama, keshi

REAL PI

$\mathrm{PI}=3.1415926$

$\mathrm{N}=1$

c gama $=$ core width/core height, for my specimen, gama $=a / b=4 / 2=2$

GAMA $=2.0$

c keshi $=0$

$\mathrm{F}=-\mathrm{FK} 1(\mathrm{X}) * \sin (\mathrm{FK} 1(\mathrm{X}) * \mathrm{~N} * \mathrm{PI} / \mathrm{GAMA} / 2). * \cos (\mathrm{FK} 2(\mathrm{X}) * \mathrm{~N} * \mathrm{PI} / \mathrm{GAMA} / 2$.

$\$+\mathrm{FK} 2(\mathrm{X}) * \sin (\mathrm{FK} 2(\mathrm{X}) * \mathrm{~N} * \mathrm{PI} / \mathrm{GAMA} / 2). * \cos (\mathrm{FK} 1(\mathrm{X}) * \mathrm{~N} * \mathrm{PI} / \mathrm{GAMA} / 2$.)

$\$$-keshi*cos(FK1(X)*N*PI/GAMA/2.)* $\cos (\mathrm{FK} 2(\mathrm{X}) * \mathrm{~N} * \mathrm{PI} / \mathrm{GAMA} / 2$.

$\$ * \mathrm{FK} 3(\mathrm{X}) * \mathrm{~N} * \mathrm{PI} / \mathrm{GAMA}$

RETURN

END

c DDHRT: Subroutine to find the solution for a function

c in a given range

c

c Parameter definition:

c A, B: Lower and upper limit of the range

c H: step length

c ESP: exquisition number

c X: vector of the solution

c $\mathrm{N}$ : predicted number of solutions in the given range

c $\mathrm{M}$ : number of solutions after calculation

c F: function $\mathrm{f}(\mathrm{x})$

SUBROUTINE DDHRT(A,B,H,EPS,X,N,M,F)

DIMENSION X(N)

$\mathrm{M}=0$

$\mathrm{Z}=\mathrm{A}$

$\mathrm{Y}=\mathrm{F}(\mathrm{Z})$

10 IF((Z.GT.B+H/2.0).OR.(M.EQ.N)) RETURN

IF(ABS(Y).LT.EPS) THEN 


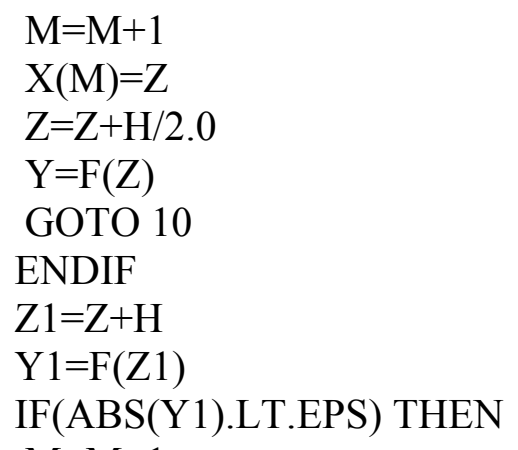

$\mathrm{M}=\mathrm{M}+1$

$\mathrm{X}(\mathrm{M})=\mathrm{Z1}$

$\mathrm{Z}=\mathrm{Z} 1+\mathrm{H} / 2.0$

$\mathrm{Y}=\mathrm{F}(\mathrm{Z})$

GOTO 10

ENDIF

IF(Y*Y1.GT.0.0) THEN

$\mathrm{Y}=\mathrm{Y} 1$

$\mathrm{Z}=\mathrm{Z} 1$

GOTO 10

ENDIF

20 IF(ABS(Z1-Z).LT.EPS) THEN

$\mathrm{M}=\mathrm{M}+1$

$\mathrm{X}(\mathrm{M})=(\mathrm{Z} 1+\mathrm{Z}) / 2.0$

$\mathrm{Z}=\mathrm{Z} 1+\mathrm{H} / 2.0$

$\mathrm{Y}=\mathrm{F}(\mathrm{Z})$

GOTO 10

ENDIF

$\mathrm{Z} 0=(\mathrm{Z}+\mathrm{Z1}) / 2.0$

$\mathrm{Y} 0=\mathrm{F}(\mathrm{Z} 0)$

IF(ABS(Y0).LT.EPS) THEN

$\mathrm{M}=\mathrm{M}+1$

$\mathrm{X}(\mathrm{M})=\mathrm{Z} 0$

$\mathrm{Z}=\mathrm{Z} 0+\mathrm{H} / 2.0$

$\mathrm{Y}=\mathrm{F}(\mathrm{Z})$

GOTO 10

ENDIF

IF(Y*Y0.LT.0.0) THEN

$\mathrm{Z} 1=\mathrm{Z} 0$

$\mathrm{Y} 1=\mathrm{Y} 0$

ELSE

$\mathrm{Z}=\mathrm{Z} 0$

$\mathrm{Y}=\mathrm{Y} 0$

ENDIF

GOTO 20

END 


\section{APPENDIX B}

\section{STRENGTH DATA OF CORE MATERIALS}

\section{Compressive Strength}

The same method as described in Chapter 5 is used to carry out the compression test on core material. The results are shown in Table B.1. A typical failure mode is given in Fig. B.1.

Table B.1 Compressive strength

\begin{tabular}{|c|c|c|c|c|}
\hline Specimen & $\begin{array}{c}\text { Ultimate Load } \\
(\mathrm{lb})\end{array}$ & $\begin{array}{c}\text { Thickness } \\
\text { (in.) }\end{array}$ & $\begin{array}{c}\text { Width } \\
\text { (in.) }\end{array}$ & $\begin{array}{c}\text { Compressive } \\
\text { strength } \\
\text { (psi) }\end{array}$ \\
\hline 1 & 6272 & 0.2805 & 1.035 & 21604 \\
\hline 2 & 6034 & 0.2785 & 1.038 & 20873 \\
\hline 3 & 6493 & 0.2935 & 1.036 & 21354 \\
\hline 4 & 6950 & 0.3 & 1.0345 & 22394 \\
\hline 5 & 6272 & 0.2795 & 1.037 & 21149 \\
\hline
\end{tabular}

Average strength: $\mathbf{2 1 , 4 7 5}$ psi

Standard deviation of strength: 580 psi 


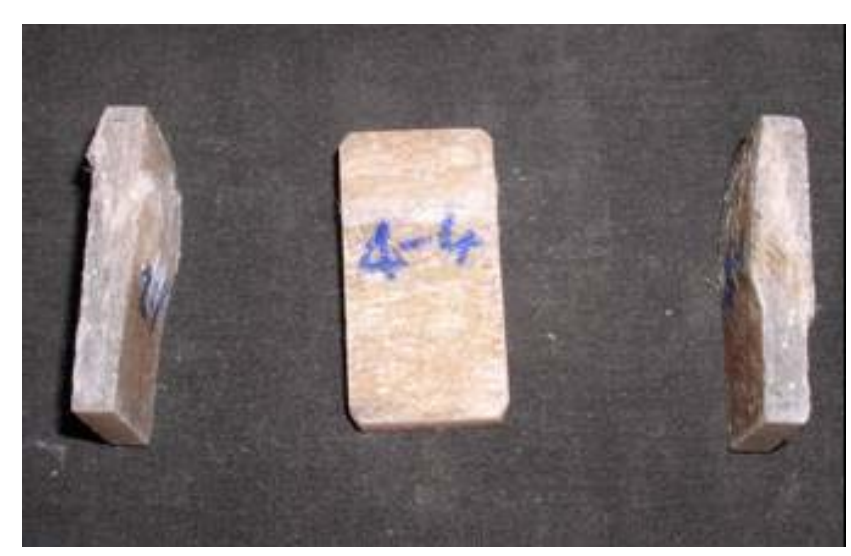

Fig B.1 Failed specimen under compression

\section{Shear Strength}

$\mathrm{V}$-notched test is used to find the shear strength. The results are given in Table B.2. A typical shear failure mode is illustrated in Fig. B.2 and the test setup is shown in Fig. B.3,

Table B.2 Shear strength

\begin{tabular}{|c|c|c|c|c|}
\hline Specimen & $\begin{array}{c}\text { Ultimate Load } \\
(\mathrm{lb})\end{array}$ & $\begin{array}{c}\text { Thickness } \\
\text { (in.) }\end{array}$ & $\begin{array}{c}\text { Width } \\
\text { (in.) }\end{array}$ & $\begin{array}{c}\text { Shear strength } \\
\text { (psi) }\end{array}$ \\
\hline 1 & 1297 & 0.269 & 0.462 & 10436 \\
\hline 2 & 1261 & 0.2785 & 0.459 & 9865 \\
\hline 3 & 1440 & 0.291 & 0.461 & 10734 \\
\hline 4 & 1298 & 0.29 & 0.4575 & 9783 \\
\hline 5 & 1367 & 0.2845 & 0.463 & 10378 \\
\hline
\end{tabular}

Average strength: $\mathbf{1 0 , 2 3 9} \mathrm{psi}$

Standard deviation of strength: 404 psi 


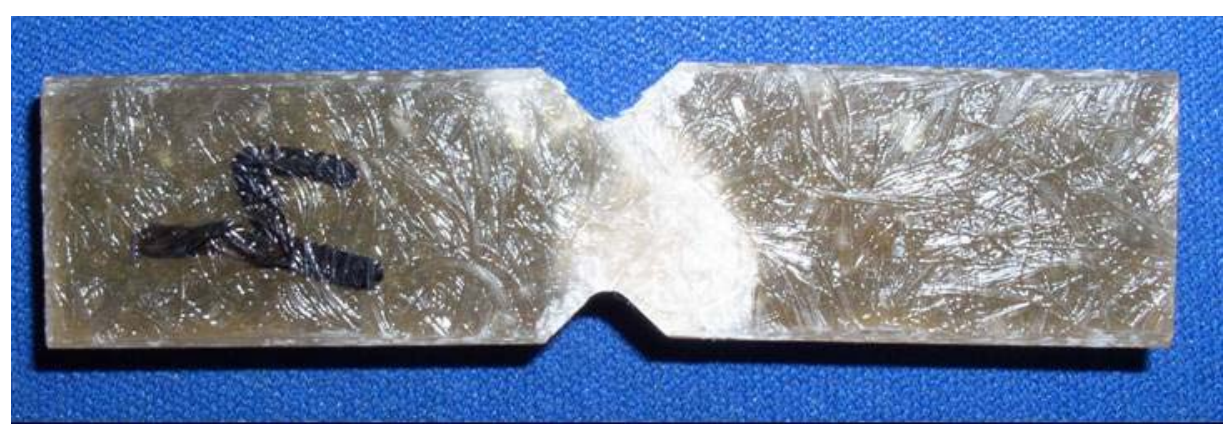

Fig. B.2 Failed specimen under shear

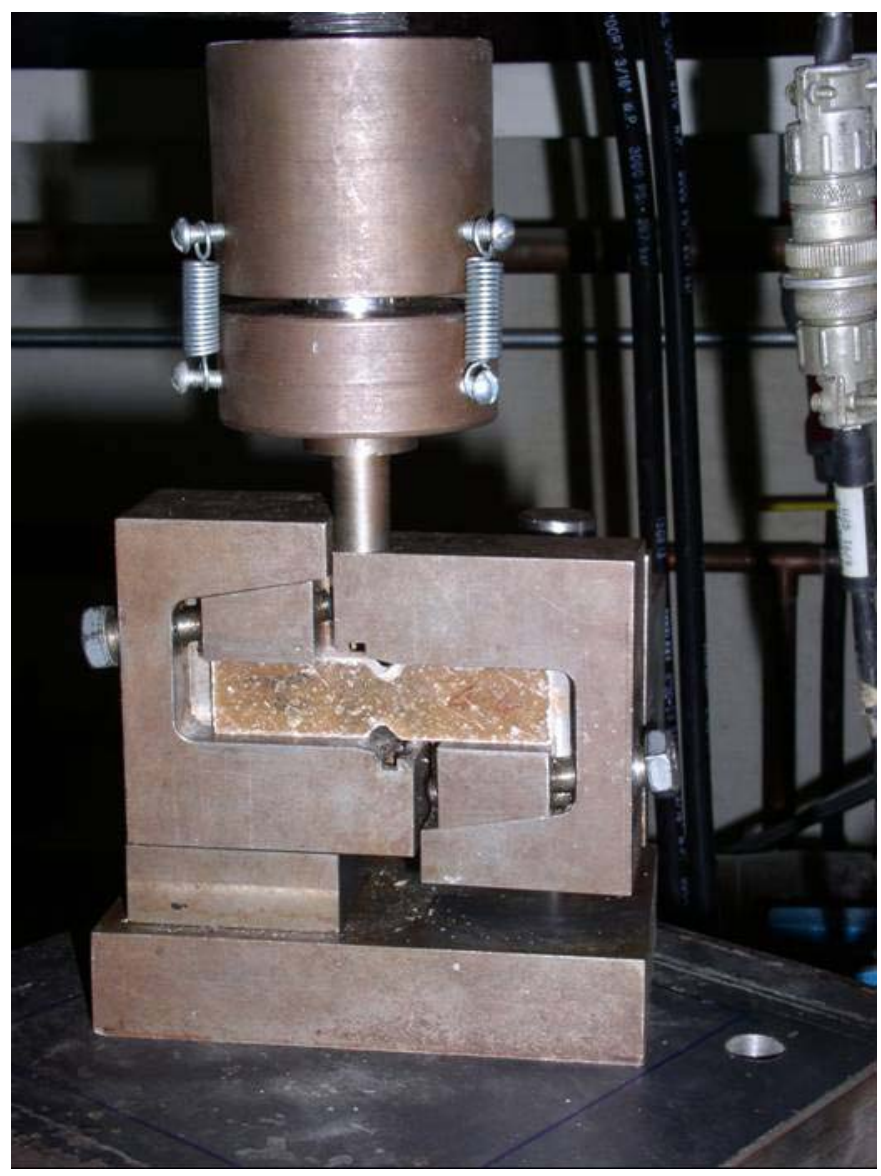

Fig. B.3 Test setup for V-notched Test 


\section{APPENDIX C}

\section{DERIVATION OF EQUILIBRIUM EQUATION}

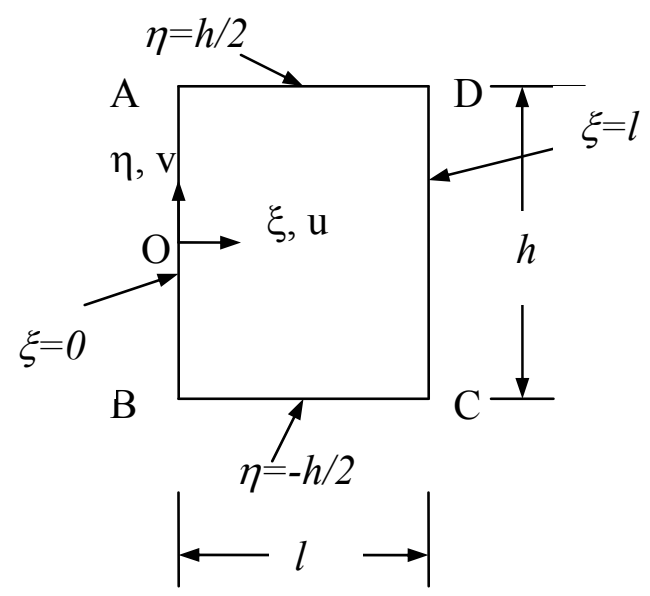

Figure C.1 Model cut from the structure

Consider the element ABCD in Fig. C.1, which is cut from the unit cell subject to a shear strain $\gamma$. The equilibrium equation for the stresses acting on the $\xi \eta$ plane in the absence of body forces is

$$
\begin{aligned}
& \frac{\partial \sigma_{\xi}}{\partial \xi}+\frac{\partial \tau_{\xi \eta}}{\partial \eta}=0 \\
& \frac{\partial \tau_{\xi \eta}}{\partial \xi}+\frac{\partial \sigma_{\eta}}{\partial \eta}=0
\end{aligned}
$$

The stress-strain relationships are 


$$
\left\{\begin{array}{c}
\sigma_{\xi} \\
\sigma_{\eta} \\
\tau_{\xi_{\eta}}
\end{array}\right\}=\left[\begin{array}{ccc}
E^{\prime} & \nu E^{\prime} & 0 \\
\nu E^{\prime} & E^{\prime} & 0 \\
0 & 0 & G
\end{array}\right]\left\{\begin{array}{c}
\varepsilon_{\xi} \\
\varepsilon_{\eta} \\
\gamma_{\xi \eta}
\end{array}\right\}
$$

where, $E^{\prime}=\frac{E}{1-v^{2}}, G=\frac{E}{2(1+v)}, E=$ Young's modulus, and $v=$ Poisson's ratio.

The strain can be found through

$$
\begin{aligned}
& \varepsilon_{\xi}=\frac{\partial u}{\partial \xi} \\
& \varepsilon_{\eta}=\frac{\partial v}{\partial \eta} \\
& \gamma_{\xi \eta}=\frac{\partial u}{\partial \eta}+\frac{\partial v}{\partial \xi}
\end{aligned}
$$

where $u$ and $v$ are the displacement in the $\xi$ and $\eta$ directions, respectively. For the consideration of shear warping, we can assume that there is no stretching in the $\xi$ direction. Then we have

$$
u=u(\eta) \quad \varepsilon_{\xi}=0
$$

Eq. (C.3) can then be reduced to 


$$
\begin{aligned}
& \sigma_{\xi}=v E^{\prime} \varepsilon_{\eta} \\
& \sigma_{\eta}=E^{\prime} \varepsilon_{\eta} \\
& \tau_{\xi \eta}=G \gamma_{\xi \eta}
\end{aligned}
$$

Differentiating Eq. (C.5b) and (C.5c) with respect to $\eta$ and $\xi$, respectively, substituting into Eq. (C.1b), and using Eqs (C.3b) and (C.3c), one obtains

$$
G \frac{\partial^{2} v}{\partial \xi^{2}}+E^{\prime} \frac{\partial^{2} v}{\partial \eta^{2}}=0
$$

From the boundary conditions shown in Fig. 4.6, $u(\eta)=0$ at both $\eta=h / 2$ and $\eta=-h / 2$, and therefore $u$ is negligible throughout the panel. Eq. (C.5) becomes

$$
\begin{aligned}
& \sigma_{\xi}=v E^{\prime}(\partial v / \partial \eta) \\
& \sigma_{\eta}=E^{\prime}(\partial v / \partial \eta) \\
& \tau_{\xi \eta}=G(\partial v / \partial \xi)
\end{aligned}
$$




\section{APPENDIX D}

\section{SHEAR TEST FOR FACESHEET LAMINATES}

\section{Experimental Setup}

Shear test (Iosipescu test) was carried out on facesheet laminates for completeness. Due to the cumbersome efforts and time needed for the specimen preparation, only two types were chosen, i.e., Laminate 1L (current configuration) and Laminate 2 (recommended configuration) from Table 5.7, with five specimens each. All specimens were sanded to be around 0.5 " thick to fit in the fixture. The dimensions of the specimen are given in Fig. D.1. The sketch of the test setup is shown in Fig. D.2, with two photos given in Fig. D.3. All tests were carried out in an MTS machine. Shear strain gage was bonded between the two V-notch, and the displacement and load were recorded using the internal displacement transducer and load cell. The loading rate was controlled at $0.05 \% / \mathrm{min}$.

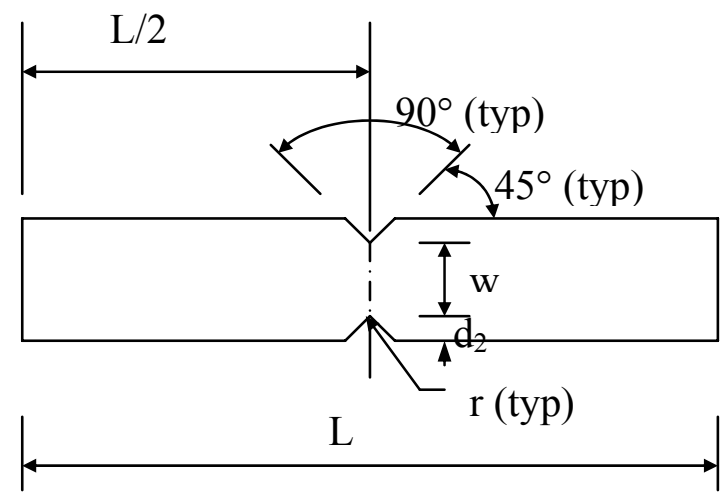

Front

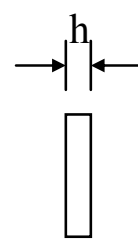

Side
Nominal Specimen

Dimensions:

$\mathrm{d}_{1}=0.75$ in.

$\mathrm{d}_{2}=0.15 \mathrm{in}$.

$\mathrm{h}=$ as required

$\mathrm{L}=3.0$ in. $\mathrm{r}=0.05 \mathrm{in}$.

Figure D.1 Specimen dimensions 


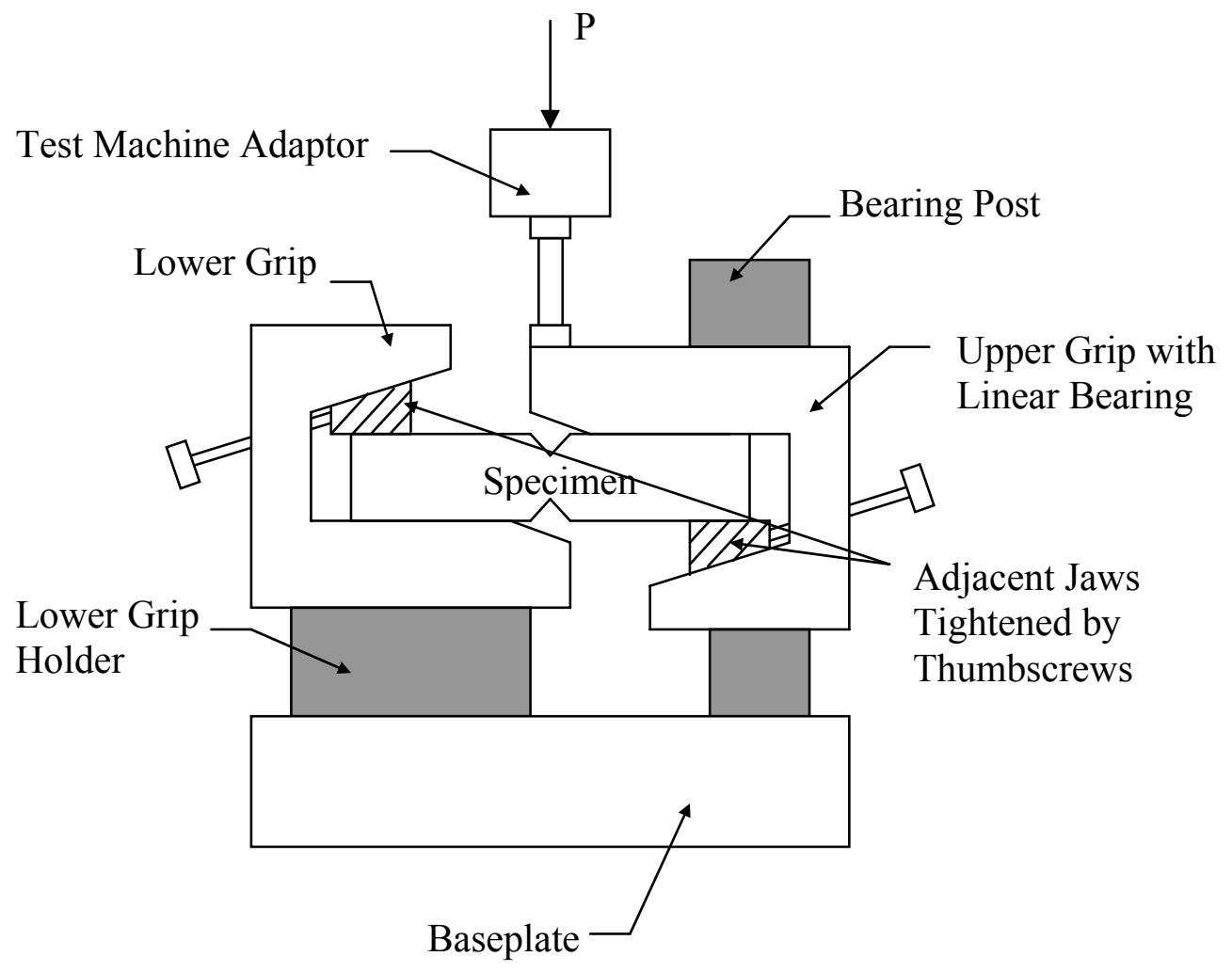

Figure D.2 Iosipescu test setup
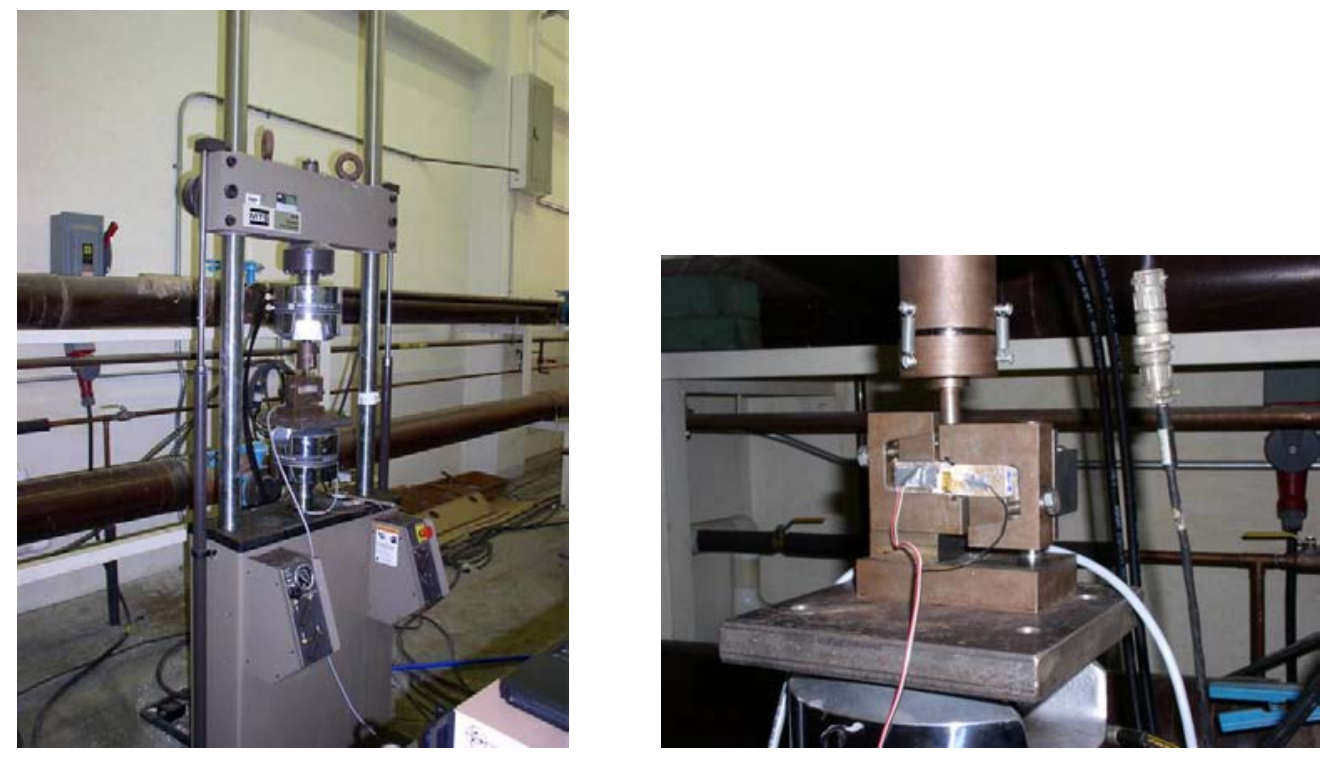

Figure D.3 Photos of test setup 


\section{Experimental Results}

All results are given in terms of applied load vs. displacement at mid-span and applied load vs. strain at mid-span, as shown in Fig. D.4 and D.5, which indicates that the specimen followed roughly linear-elastic behaviour before the failure occurred at Vnotched area. After the specimens failed, the load dropped slowly until the displacement reached the capacity of the testing fixture. Fig. D.6 demonstrates several failed specimens, showing a typical shear failure.

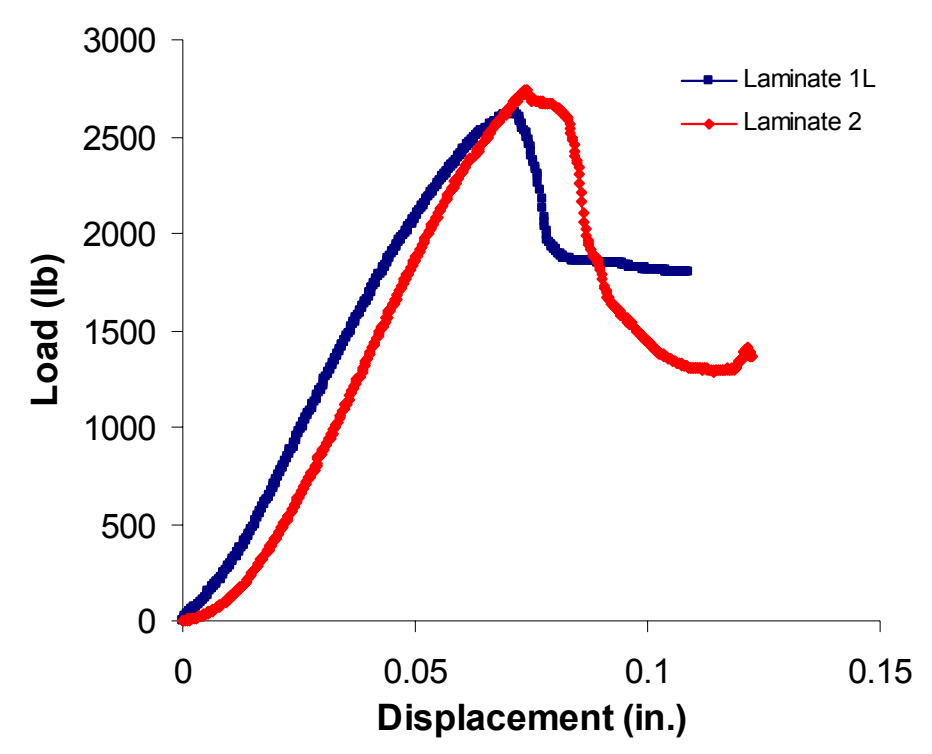

Fig. D.4 Load-displacement curves 


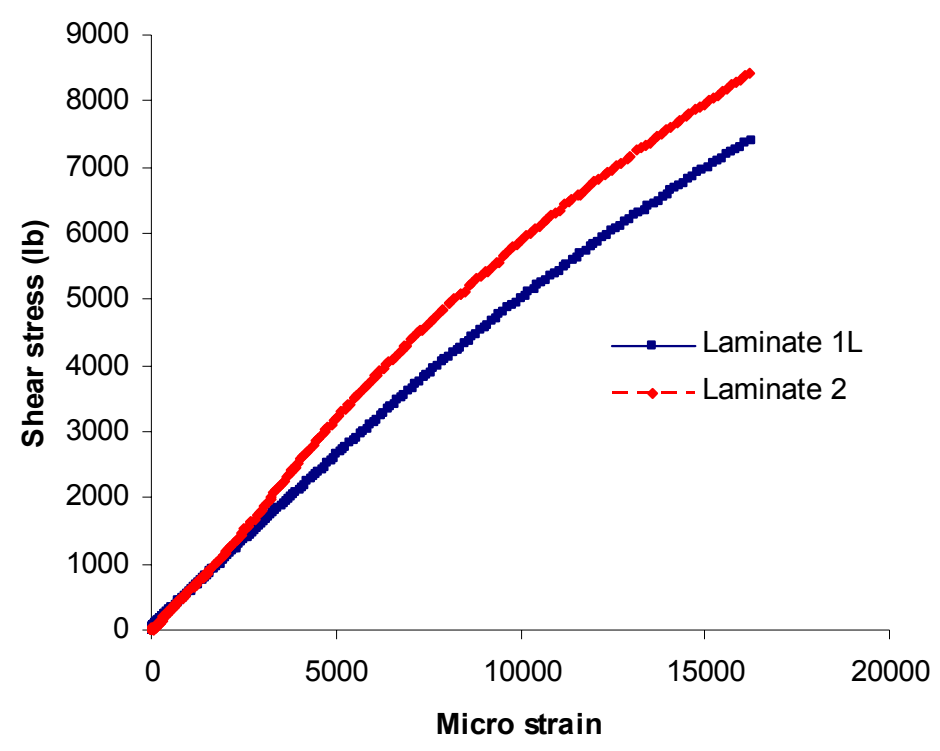

Fig. D.5 Load-strain curves
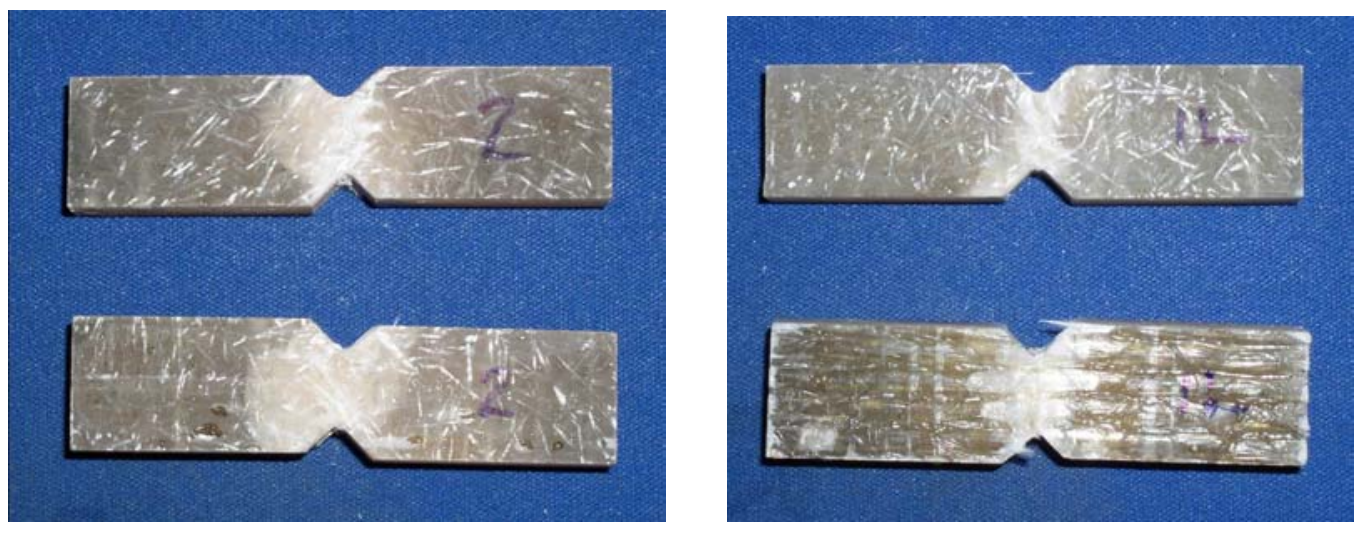

Figure D.6 Failure mode

The shear strength for each specimen of Laminate 1L and 2 are given in Table D.1 and D.2, respectively, from which we can see that, when ChSM layers are introduced into the facesheet laminate, the shear strength becomes slightly higher, due to the low inplane shear strength of fibers. 
Table D.1 Shear strength for Laminate 1L

\begin{tabular}{|c|c|c|c|c|}
\hline Specimen & $\begin{array}{c}\text { Ultimate Load } \\
(\mathrm{lb})\end{array}$ & $\begin{array}{c}\text { Thickness } \\
\text { (in.) }\end{array}$ & $\begin{array}{c}\text { Width } \\
\text { (in.) }\end{array}$ & $\begin{array}{c}\text { Shear strength } \\
\text { (psi) }\end{array}$ \\
\hline 1 & 2528 & 0.492 & 0.438 & 11731 \\
\hline 2 & 2690 & 0.4925 & 0.446 & 12246 \\
\hline 3 & 2564 & 0.493 & 0.45 & 11557 \\
\hline 4 & 2424 & 0.4795 & 0.44 & 11489 \\
\hline 5 & 2727 & 0.49 & 0.445 & 12506 \\
\hline
\end{tabular}

Average strength: $\mathbf{1 1 , 9 0 6}$ psi

Standard deviation of strength: 448 psi

Table B.2 Shear strength for Laminate 2

\begin{tabular}{|c|c|c|c|c|}
\hline Specimen & $\begin{array}{c}\text { Ultimate Load } \\
(\mathrm{lb})\end{array}$ & $\begin{array}{c}\text { Thickness } \\
\text { (in.) }\end{array}$ & $\begin{array}{c}\text { Width } \\
\text { (in.) }\end{array}$ & $\begin{array}{c}\text { Shear strength } \\
\text { (psi) }\end{array}$ \\
\hline 1 & 2781 & 0.493 & 0.4385 & 12,864 \\
\hline 2 & 2883 & 0.491 & 0.4225 & 13,897 \\
\hline 3 & 2889 & 0.492 & 0.448 & 13,107 \\
\hline 4 & 2744 & 0.487 & 0.433 & 13,012 \\
\hline 5 & 2701 & 0.491 & 0.4455 & 12,347 \\
\hline
\end{tabular}

Average strength: 13,046 psi

Standard deviation of strength: 559 psi 


\section{APPENDIX E}

\section{STIFFNESS OF FACESHEET LAMINATES AND CORE MATERIALS}

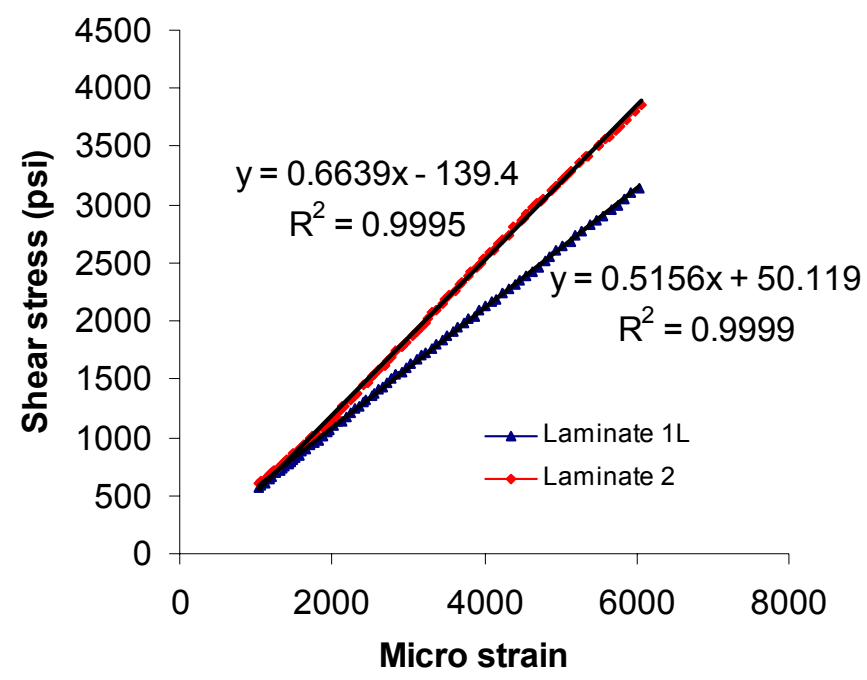

Figure E.1 Stress-strain curves to find shear stiffness

Based on the strain and stress data collected from the compression and shear tests on core materials and facehsheet laminates (Table 5.7), the stiffness can be obtained by fitting the data between 1000 and 6000 micro strain, with one example shown in Fig. E.1 for determining shear stiffness. The results based on two specimens for each type are listed in Table E.1.

Table E.1 Stiffness of facesheet laminates and core materials

\begin{tabular}{|c|c|c|}
\hline Type & $\begin{array}{c}\text { Shear modulus } \\
\left(\times 10^{6} \mathrm{psi}\right)\end{array}$ & $\begin{array}{c}\text { Compressive stiffness } \\
\left(\mathrm{x} 10^{6} \mathrm{psi}\right)\end{array}$ \\
\hline $1 \mathrm{~L}$ & 0.5156 & 2.4173 \\
\hline $1 \mathrm{~T}$ & - & 1.5716 \\
\hline 2 & 0.6639 & 1.8866 \\
\hline 3 & - & 2.3209 \\
\hline Core materials & 0.5607 & 1.3280 \\
\hline
\end{tabular}

